\title{
Modeling and Simulation of Microstructure Evolution and Deformation in an Irradiated Environment
}

Stephanie A. Pitts

April 2019

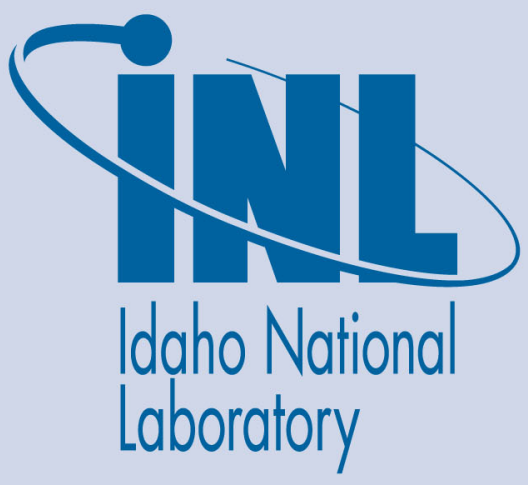

The INL is a U.S. Department of Energy National Laboratory operated by Battelle Energy Alliance 


\title{
Modeling and Simulation of Microstructure Evolution and Deformation in an Irradiated Environment
}

\author{
Stephanie A. Pitts
}

April 2019

Idaho National Laboratory Idaho Falls, Idaho 83415

http://www.inl.gov

Prepared for the U.S. Department of Energy

Office of Nuclear Energy, Office of Nuclear Energy, Office of Nuclear Energy Under DOE Idaho Operations Office

Contract DE-AC07-05ID14517, DE-AC07-05ID14517, DE-AC07-05ID14517 


\section{MODELING AND SIMULATION OF MICROSTRUCTURE EVOLUTION AND DEFORMATION IN AN IRRADIATED ENVIRONMENT}

By

STEPHANIE ANNE PITTS

A dissertation submitted in partial fulfillment of the requirements for the degree of

DOCTOR OF PHILOSOPHY

WASHINGTON STATE UNIVERSITY

School of Mechanical and Materials Engineering

MAY 2019

(c) Copyright by STEPHANIE ANNE PITTS, 2019

All Rights Reserved 
(C) Copyright by STEPHANIE ANNE PITTS, 2019

All Rights Reserved 
To the Faculty of Washington State University:

The members of the Committee appointed to examine the dissertation of STEPHANIE ANNE PITTS find it satisfactory and recommend that it be accepted.

Hussein M. Zbib, Ph.D., Chair

Erin I. Barker, Ph.D.

David P. Field, Ph.D.

Sinisa Mesarovic, Ph.D. 


\section{ACKNOWLEDGMENTS}

I wish to thank the Bison, MOOSE, and Marmot teams at Idaho National Laboratory who have given me support, encouraging advice, and time for discussions which have all helped to complete this work. In particular I wish to thank Cody Permann, Wen Jiang, Davide Pizzocri, Gary Hu, Matthias Kunick, Daniel Schwen, and Ben Spencer for the assistance with and discussion about code implementation; to Steve Novascone, Al Casagranda, and Larry Aagensen for the discussions about FEM basics, shape functions, and code verification; to Russell Gardner, Andrew Slaughter, and Kyle Gamble for the advice on postprocessing and data visualization; to Jason Hales and Rich Williamson for their mentorship and the support and encouragement to complete this thesis.

Secondly, I wish to thank my adviser, Dr. Zbib, and my committee members, Dr. Barker, Dr. Field, and Dr. Mesarovic for their willingness to share their knowledge of microstructural evolution and modeling with me. I am grateful to Dr. Zbib for first introducing me to the fascinating subject of dislocations and for his advice and patience over the years; to Dr. Barker for introducing me to MOOSE and to code development with in a national laboratory; to Dr. Field for helping me to understand 
experimental measurements of microstructure and for introducing me to material applications in nuclear power plants; to Dr. Mesarovic for his willingness to teach me continuum mechanics despite the numerous office hours spent on Einstein notation.

Finally I would like to acknowledge the support and encouragement I received from the ladies at Clockwork Farms: from the offers to pamper my pony when I was struggling with a simulation to the antics that ensued when I asked for volunteers to proofread my thesis - thank you.

Portions of this manuscript include work performed by the author while a contractor of the U.S. Government under Contract DE-AC07-05ID14517. Accordingly, the U.S. Government retains a non-exclusive, royalty free license to publish or reproduce the published form of this contribution, or allow others to do so, for U.S. Government purposes. Additionally, this research made use of the resources of the High Performance Computing Center at Idaho National Laboratory, which is supported by the Office of Nuclear Energy of the U.S. Department of Energy and the Nuclear Science User Facilities under Contract No. DE-AC07-05ID14517. 


\title{
MODELING AND SIMULATION OF MICROSTRUCTURE EVOLUTION AND DEFORMATION IN AN IRRADIATED ENVIRONMENT
}

\author{
Abstract \\ by Stephanie Anne Pitts, Ph.D. \\ Washington State University \\ May 2019
}

Chair: Hussein M. Zbib

The ability to predict the behavior of structural components in a nuclear power plant is critical to the nuclear industry. Structural metals in the primary loop of nuclear power plants must endure challenges such as irradiation and mechanical and thermal loading, and these structural metal components must continue to function in potential transient and accident conditions throughout the operational lifetime of the power plant. This extreme operational environment changes the metal microstructure by creating additional defects. The physical interactions of dislocations with these defects govern how the metal will respond to future conditions. Therefore predicting the mechanical response of these metals requires a set of physically based and reliable models of dislocation and defect interactions. These microstructure elements 
include glide mobile and immobile dislocations, geometrically necessary dislocations, twinning dislocations, irradiation defects, and thermal aging defects. We present here a continuum dislocation dynamics crystal plasticity framework to capture the interaction mechanisms of these dislocations and defects, verified with a combination of benchmark problems and comparisons with experimental data for two different types of structural metals: $\alpha$ iron and nickel-based alloys. In our simulations of $\alpha$ iron we highlight the advantages of applying a Monte Carlo stochastic model of cross slip dislocation motion and show the importance of capturing the 3D nature of glide dislocation and self-interstitial atom loop radiation defect interactions. We demonstrate coupling of glide dislocations with geometrically necessary dislocations to capture the influence of lattice bending, including the sensitivity of the geometrically necessary dislocations to changes in the grain boundary angle. We further examine the interaction of glide dislocations with the twin dislocations and thermally aged defects which have been observed in a nickel-based alloy with additional models. Finally we assess the reliability of this crystal plasticity framework by comparing two dislocation glide velocity models across the range of normal operation temperatures. In successfully applying our crystal plasticity framework to multiple metals, we provide further evidence of the reliability of our approach. The results of this mechanismbased continuum dislocation dynamics crystal plasticity framework can be used to inform engineering scale models throughout the nuclear industry. 


\section{TABLE OF CONTENTS}

Page

ACKNOWLEDGMENTS $\ldots \ldots \ldots \ldots \ldots \ldots \ldots \ldots \ldots \ldots \ldots \ldots \ldots \ldots \ldots \ldots \ldots \ldots \ldots \ldots$

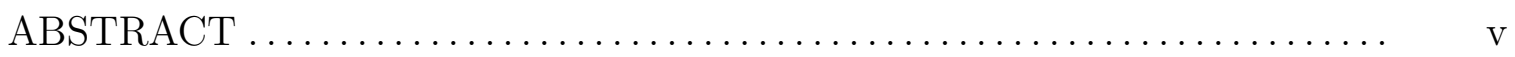

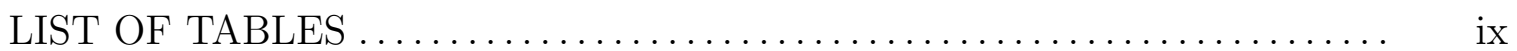

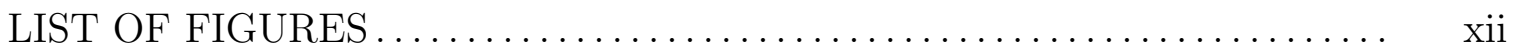

LIST OF ACRONYMS $\ldots \ldots \ldots \ldots \ldots \ldots \ldots \ldots \ldots \ldots \ldots \ldots \ldots \ldots \ldots \ldots \ldots \ldots \ldots \ldots \ldots \ldots$

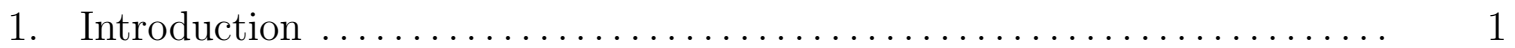

1.1 Evolution of Microstructure under Irradiation $\ldots \ldots \ldots \ldots \ldots \ldots \ldots$

1.2 Crystal Plasticity Models of Microstructure Evolution ............. 8

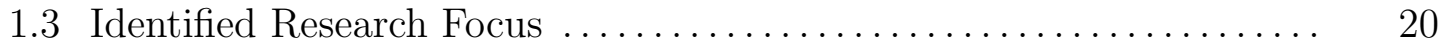

1.4 Overview of Thesis ................................... 22

2. Theoretical Overview and Numerical Implementation $\ldots \ldots \ldots \ldots \ldots \ldots \ldots . \ldots \ldots$

2.1 Continuum Solid Mechanics Overview ................... 26

2.2 Crystalline Structure of Metals . . . . . . . . . . . . . . . . 49

2.3 Crystal Plasticity Framework ....................... 67

2.4 Numerical Implementation in MOOSE................... 78

3. Continuum Dislocation Dynamics Model for Irradiated $\alpha$-Iron . . . . . . . 83

3.1 CDD Crystal Plasticity Implementation for BCC $\alpha$-Iron . . . . . . . $\quad 85$

3.2 Single Crystal $\alpha$-Iron CDD Simulations .................... 89

3.3 Comparison of Stochastic Cross Slip Models .................... 101

3.4 Polycrystalline Irradiated $\alpha$-Iron Simulations $\ldots \ldots \ldots \ldots \ldots \ldots \ldots \ldots$

3.5 Conclusions and Future Recommendations.................... 112 
4. Geometrically Necessary Dislocation Density Model Addition........... 114

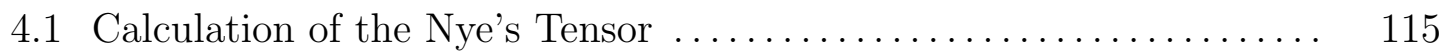

4.2 Geometrically Necessary Dislocation Density Measure............ 125

4.3 Thin Single Crystal Beam Bending Benchmark Problem .......... 127

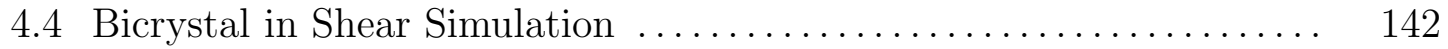

4.5 Conclusions and Future Recommendations.................. 153

5. Twinning Volume Evolution Model Addition ..................... 155

5.1 Twinning Model Implementation . . . . . . . . . . . . . . . . . . . . . 160

5.2 Single Crystal CDD with Twinning Simulations................. 165

5.3 Micropillar Compression Nickel-Chromium Simulations .......... 174

5.4 Conclusions and Future Recommendations................... 186

6. Dislocation Glide Velocity Model Comparison ..................... 188

6.1 Formulation of Dislocation Glide Velocity Equations .............. 190

6.2 Single Crystal $\alpha$ Iron Simulations $\ldots \ldots \ldots \ldots \ldots \ldots \ldots \ldots \ldots \ldots$

6.3 Conclusions and Recommendations ....................... 221

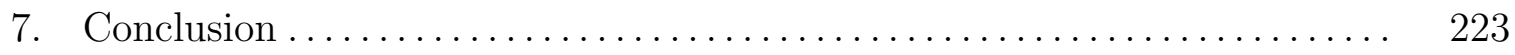

7.1 Future Development and Potential Applications ............... 225

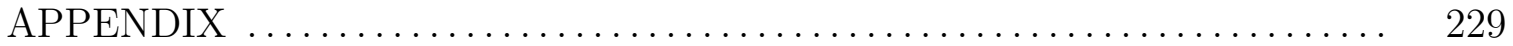

A. CDD Crystal Plasticity Classes Implemented in MOOSE .......... 229

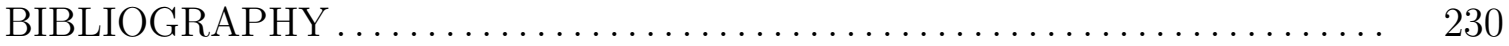




\section{LIST OF TABLES}

Table Page

2.1 Glide slip systems in BCC crystals $\ldots \ldots \ldots \ldots \ldots \ldots \ldots \ldots \ldots \ldots \ldots \ldots \ldots \ldots \ldots$

2.2 Glide slip systems in FCC crystals $\ldots \ldots \ldots \ldots \ldots \ldots \ldots \ldots \ldots \ldots \ldots$

2.3 Cross slip families in BCC crystals $\ldots \ldots \ldots \ldots \ldots \ldots \ldots \ldots \ldots \ldots \ldots \ldots$

2.4 Mathematical expressions for the six physically-based interaction mechanisms used within the mobile dislocation evolution equation, Eq (2.67), and immobile dislocation evolution equation, Eq (2.68). The six $\alpha$ coefficients are fit from dislocation dynamics simulations [101], $v_{\text {glide }}$ is the dislocation glide velocity from $\mathrm{Eq}(2.65), R_{c}$ is the radius of capture for annihilating dislocations of opposite sign, and $l_{i n v}$ is the inverse mean free glide path. $\ldots \ldots \ldots \ldots \ldots \ldots \ldots \ldots \ldots . \ldots \ldots$

2.5 Twinning systems in FCC crystals $\ldots \ldots \ldots \ldots \ldots \ldots \ldots \ldots \ldots \ldots \ldots$

3.1 Parameter values used in the single crystal CDD $\alpha$-Fe simulations. . $\quad 90$

3.2 Dislocation evolution parameters for Eqs (2.67) and (2.68) and Table 2.4 calibrated for the crystal plasticity model in single crystal $\alpha$-Fe

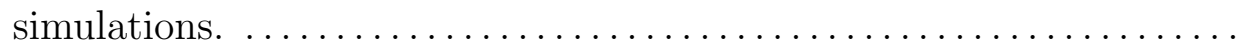

3.3 Values of the parameters used in the CDD crystal plasticity model in the SIA loop terms, Eqs (2.73) and (2.72), for the verification of

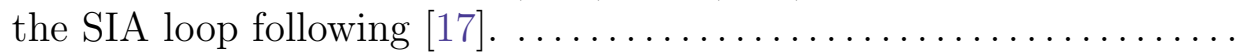

3.4 Values of the parameters used in the SIA loop terms of the crystal plasticity model for polycrystalline $\alpha$-Fe exposed to a radiation dose of $0.1 \mathrm{dpa}$, including initial conditions. ................. 108

4.1 The set of varied $y$ dimension depths of the thin beam, Figure 4.2, where the length of the cubic Hex8 elements is determined by the beam depth. The corresponding number of elements in the $x$ direction width and in the $z$ direction height are also given. ..........

4.2 Constant material parameter values used in the single crystal nickel simulations of the thin beam bending benchmark. 
4.3 Values of the dislocation evolution parameters used in the thin beam bending simulations to compare the approaches of calculating the plastic velocity gradient derivative. These parameters, for the CDD dislocation evolution model, Eq (2.67) and Eq (2.68), are appropriate for an FCC material and are slight modified from the values determined by [177] for nickel based on fitting conducted as supplemental

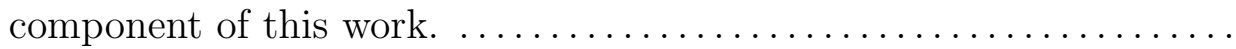

4.4 The set of Bunge Euler angles used in the set of three simulations of the bicrystal problem, taken from [108, 109]. The geometry of the bicrystal problem is shown in Figure 4.8, and the notation top grain and bottom grain correspond to the orientation of the bicrystal as

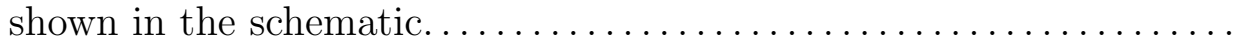

4.5 Constant material parameter values used in the aluminum sheared bicrystal benchmark simulations. .......................

4.6 Values of the dislocation evolution parameters used in the simulations of the sheared bicrystal, Figure 4.8. These parameters are for the CDD dislocation evolution model, Eq (2.67) and Eq (2.68) and are fit for FCC aluminum $[177] \ldots \ldots \ldots \ldots \ldots \ldots \ldots \ldots \ldots \ldots$

5.1 The elasticity tensor and hardening material parameter values used in the CDD crystal plasticity for twinning model addition simula-

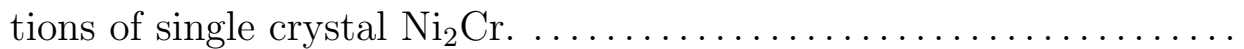

5.2 Values of the dislocation evolution parameters used in the simulations for the single crystal twinning model verification benchmark simulations. These parameters are for the CDD dislocation evolution model, Eq (2.67) and Eq (2.68) and are fit for FCC aluminum [177], and for the twinning evolution model. ................

5.3 The elasticity tensor and hardening material parameter values used in the CDD crystal plasticity for the micropillar $\mathrm{Ni}_{2} \mathrm{Cr}$ simulations. The characteristics of the LRO precipitates for the aged micropillar simulations are also listed.

6.1 Constant material parameter values used in the single crystal $\alpha$-Fe simulations for the comparison of dislocation glide velocity models. . 
6.2 Values of the dislocation evolution parameters used in the simulations comparing dislocation glide velocity equations. These parameters were fit to single crystal $\alpha$-Iron experimental data in Section 3.2.1 for the CDD dislocation evolution model, Eq (2.67) and Eq (2.68) and are appropriate for loading in the [100] direction. .......

6.3 Parameter values for the power law dislocation glide velocity model, Eq (6.1) in the temperature variation comparison of dislocation glide velocity model, taken from our work in Section 3.2.1. The initial dislocation glide velocity is calculated through Eq (6.2) from a reference shear rate of $4.0 \times 10^{-2}[101]$, as given in Table $3.1 \ldots \ldots \ldots \ldots \ldots \ldots$

6.4 Parameter values for the enthalpy-based dislocation glide velocity model, Eq (6.1) in the temperature variation comparison of dislocation glide velocity model, from $[28] . \ldots \ldots \ldots \ldots \ldots \ldots \ldots \ldots \ldots$

6.5 Set of temperature values, spanning the typical range of temperatures experienced by the reactor pressure vessel for normal operating conditions to shutdown [40], used in the temperature study portion of the dislocation glide velocity model comparison.............

6.6 Variations of the exponential constant in the power law dislocation glide velocity model, Eq (6.1) used in the coefficient perturbation

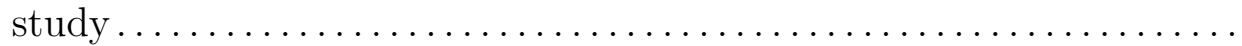

6.7 Variation of exponential constants in the enthalpy-based dislocation glide velocity model, Eq (6.3), at a constant temperature of $25^{\circ} \mathrm{C}$,

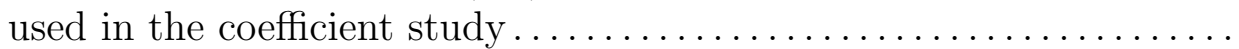

6.8 Variation of exponential constants in the enthalpy-based dislocation glide velocity model, $\mathrm{Eq}(6.3)$, at a constant temperature of $150^{\circ} \mathrm{C}$,

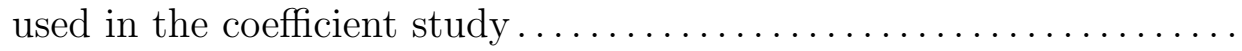




\section{LIST OF FIGURES}

Figure

Page

2.1 Example of a deformed continuum solid body with the position of a

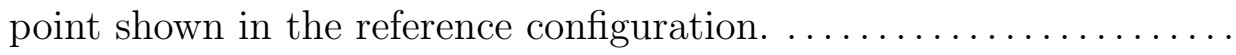

2.2 Example of a continuum solid body in both the reference and current configurations with the line elements labeled.................

2.3 The Frank-Read dislocation generation source, line (a), operates under an applied shear stress which causes the pinned dislocation to bow out, line (b), until the line at (c) is minimized. At that point, a new dislocation loop, line $(\mathrm{d})$, is released and the dislocation source,

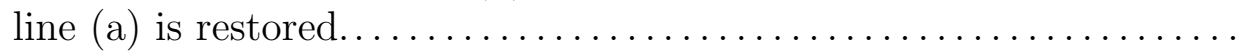

2.4 The flowchart for the calculation of the stress and strain measures within the CrystalPlasticityUpdate class as implemented in the tensor mechanics module of MOOSE is shown here. The components involved in the Newton-Rhapson iteration are shown in light blue and expanded in Figure 2.5, and the components shown in light orange

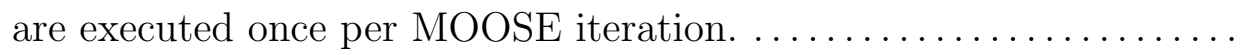

2.5 The algorithm components of the Newton-Rhapson iteration are shown in light blue and the crystal plasticity constitutive model com-

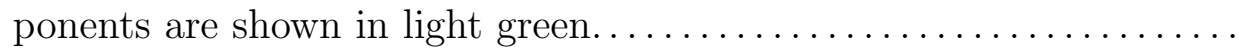

3.1 Verification of the dislocation evolution parameters listed in Table 3.2 against single crystal BCC $\alpha$-Fe tensile experimental data [78], reproduced from $[99] . \ldots \ldots \ldots \ldots \ldots \ldots \ldots \ldots \ldots \ldots \ldots \ldots \ldots \ldots \ldots \ldots \ldots \ldots$

3.2 Mesh convergence of the CDD crystal plasticity model depends to a degree on the number of active slip systems for the given loading direction. The loading direction with the greatest number of active slip systems, [100], demonstrated no mesh dependence while the single activated slip system loading orientation, [348], does demonstrate sensitivity to the number of elements used in the mesh. The numbers given in the legends correspond to the total number of elements

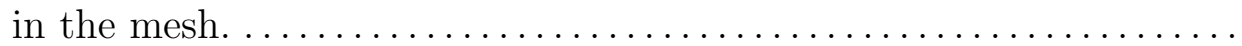


3.3 The CDD crystal plasticity model demonstrates mild sensitivity to the applied strain rate, with higher strain rates producing a higher

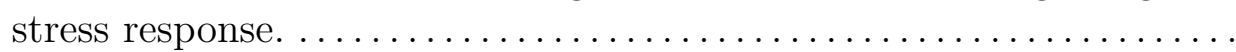

3.4 The anisotropy correction to the intrinsic lattice friction strength, Eq (3.2), hardens the $\{110\}$ type systems relative to the $\{112\}$ type systems so that dislocations can cross slip from the lower slip resistance systems to the higher resistance systems without additional slip system activation. This figure includes the six slip systems from

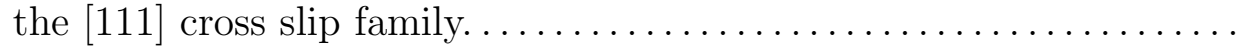

3.5 Comparison of crystal plasticity simulations with dislocation dynamics simulations of the the stress-plastic strain evolution with varying initial values of SIA loop densities demonstrate similar trends an in [17]. The dislocation dynamics data, indicated by the outlined markers, are reproduced from [8] and our crystal plasticity simulation results are shown by the solid lines....................

3.6 Comparison of the cross slip approaches on the dislocation density

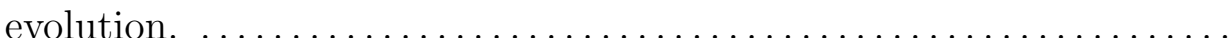

3.7 The simplified geometry, consisting of 27 cubic grains, used to examine the CDD crystal plasticity model in a polycrstalline application.

3.8 Crystal orientations of the 27 cubic grains in our simplified polycrystalline model, given in Bunge Euler angles, generated using the random function from the python library [153]. The orientation of these polycrystalline models matches the orientation shown in Figure

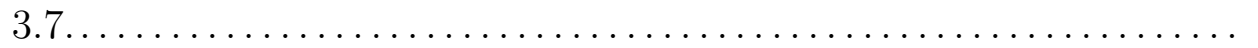

3.9 The CDD model captures the polycrstalline stress response of unirradiated and 0.1 dpa irradiated $\alpha$-iron with the simplified cubic polycrstalline geometry. The experimental data points for $\alpha$-iron are

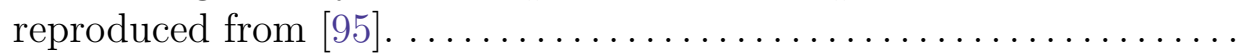

3.10 An examination of the elements near the grain boundaries in the simplified cubic polycrystalline model of $\alpha$-iron reveals a buildup of stress along some boundaries of the grains, with a higher effective stress value along certain boundaries than within the remainder of the grain. The inner boundary of the top grain and the bottom boundary of the lower grain in (a) demonstrate this observed disparity among the inner grain and the grain boundaries............. 
3.11 The distribution of the mobile dislocations on the [112](11) slip system shows the influence neighboring grains on dislocation evolution even in the local CDD model. These results are shown on the unirradiated $\alpha$ iron simplified polycrstalline geometry. ...........

4.1 A midplane 2D representation of the bottom layer of the Hex8 elements and the quadrature points (in outline) used to recover the plasticity velocity gradient at the central node of interest (in red). . .

4.2 A representation of the thin beam benchmark geometry used to evaluate the evolution of GND density in the CDD crystal plasticity model, with displacement boundary conditions shown at the top of

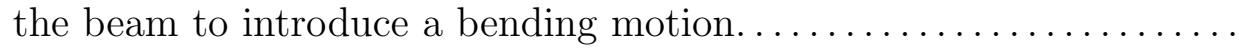

4.3 The internal shape function sum method, discussed in Section 4.1.1, shows good convergence in the solution of the effective stress, Figure 4.3a, but only poor convergence in the solution of the GND density. The calculated GND density is numerically zero. Nonetheless, in examining the pattern of the low GND densities, we note the increasing divergence of the calculated GND density at the edges of the beam, Figure 4.3b, as an indication of the mesh dependence of this approach. These results are taken across the width of the beam at half of the beam height and correspond to the final bending load

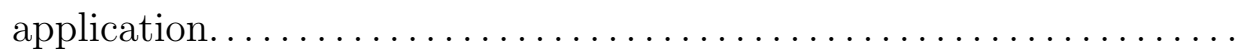

4.4 While the effective stress demonstrates the classical smooth stress distribution pattern expected in a beam bending problem, the GND density calculated with the internal shape function sum method demonstrates significant variation and pixelation. These results are shown for the $0.005 \mathrm{~mm}$ depth beam under the final bending load. The beam displacement shown in these figures has been scaled by a factor of 100 for clarity. The original beam geometry is shown in

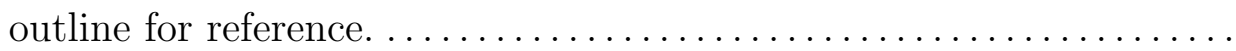


4.5 The Nodal Patch Recovery method, discussed in Section 4.1.2, shows good convergence in the solution of the effective second Piola-Kirchhoff stress, Figure 4.5a, and acceptable convergence in the GND density at the edges of the beam, Figure 4.5b. The general convergence of the GND density calculated with this method is an indication of the measure of mesh independence provided by the nodal patch recovery method. These results are taken across the width of the beam at half of the beam height and correspond to the final bending load

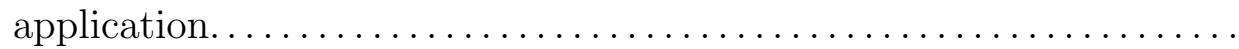

4.6 Both the effective second Piola-Kirchhoff stress and the GND density distribution demonstrate smooth distributions across the $0.005 \mathrm{~mm}$ depth beam, with the higher values for both quantities occuring on the edges of the beam as expected. The beam displacement shown in these figures has been scaled by a factor of 100 for clarity. The original beam geometry is shown in outline for reference. .........

4.7 The comparison of the Nodal Patch Recovery and Internal Shape Function Sum methods demonstrate a significant difference in calculated quantities of interest, on beams with a depth of $0.005 \mathrm{~mm}$. These results are sampled across the beam at half of the beam height under the final bending displacement. $\ldots \ldots \ldots \ldots \ldots \ldots \ldots \ldots$

4.8 The bicrystal problem, adapted from Ma et al. [109], with the grain boundary located on the midway vertical plane. In addition to the shear loading in the $\mathrm{x}$-direction shown, the displacements in the $\mathrm{y}$ and $\mathrm{z}$-directions are fixed on the top and bottom surfaces. ........

4.9 The effective second Piola-Kirchhoff stress, shown on the midplane of the bicrystal geometry $(z=1.0 \mathrm{~mm})$, demonstrates little change across the center of the bicrystal geometry among the three different misorientation angle simulations, see Table 4.4. These presented results are sampled at the onset of plasticity; thus any variation in the effective stress profiles across the three simulations due to the GND density, Figure 4.10, may not yet be observable. .......... 
4.10 The variation of the GND density is evident with the increasing grain boundary misorientation angle, even at the onset of plasticity. In the single crystal, Figure 4.10a, the GND density is concentrated at the lateral edges, while in the bicrystals the concentration of GND density is higher in the regions away from the boundary. In the small grain boundary angle simulation, Figure 4.10b, the GNDs cluster in the corners. The concentration of GND density clusters in both grains is larger in the medium grain boundary angle simulation, Figure $4.10 \mathrm{c}$. These results are from the onset of plasticity. ..........

5.1 The twinning model addition to the CDD crystal plasticity framework demonstrates the formation of twins in the loading directions known to form twins in FCC materials: compression loading in [100] and tensile loading in [111]. As observed in experimental tests, tensile loading in the [100] direction and compression loading in the [111] direction do not form twins. These results indicate the proper

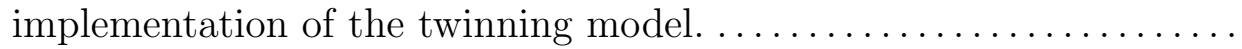

5.2 The stress response of the CDD crystal plasticity simulations with the twinning model addition does not demonstrate mesh dependence under compression, Figure 5.2a, nor in tension, Figure 5.2c. Only under compression does the total twin volume demonstrate mesh sensitivity on the coarsest mesh, Figure 5.2b. Note that the scales used for Figures 5.2b and $5.2 \mathrm{~d}$ are not equivalent. $\ldots \ldots \ldots \ldots \ldots$

5.3 As in the [100] loading direction, the response of the crystal plasticity simulations with the twinning model addition demonstrate little mesh sensitivity under tensile loading which promotes twinning growth, Figure 5.3c and 5.3d. Under compression the stress response, Figure 5.3a, and the twin volume, Figure 5.3b, demonstrate mesh sensitivity, particularly on the coarser two meshes. ..............

5.4 The micropillar compression test geometry used to run simulations of the micropillar compression tests on the binary $\mathrm{Ni}_{2} \mathrm{Cr}$ model alloy. The micropillar width and depth have the same dimension, and the substrate width and depth are the same length. The bottom of the micropillar substrate is fixed in the $x$ direction, the front and back sides of the substrate cube are fixed in the $y$ direction as are the right and left sides fixed in the $z$ direction, and a compressive displacement

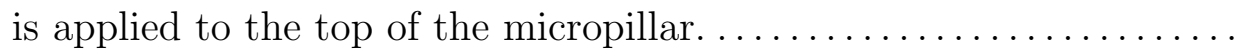


5.5 The significant differences in the force measured during the experimental micropillar compression tests and the force predicted by the crystal plasticity simulations indicates that not all of the relevant physics active in the micropillar are captured by our bulk material crystal plasticity model. We suggest further developments to the model or experimental testing of larger diameter micropillars to bring the measured and simulation results into alignment. ........

5.6 In the unaged micropillar simulations the twinning behavior aligns with expectations for twins in only the [100] orientation; however, the thermally aged LRO precipitates appear to promote some twinning in both orientations of the micropillar simulations.............

5.7 The evolution of mobile dislocations within the substrate at the base of the pillar demonstrate the importance of including the model of the substrate in our micropillar crystal plasticity simulations. Note the that scales used in Figures 5.7a and 5.7b are not equivalent. ....

6.1 The power law glide velocity model does not demonstrate large changes in the stress response or in the mobile dislocation evolution to changes in temperature; however, the observed changes are consistent with expectations for increasing temperature. The power law glide velocity model consistently demonstrates lower stresses and lower mobile dislocation densities with increasing temperature values

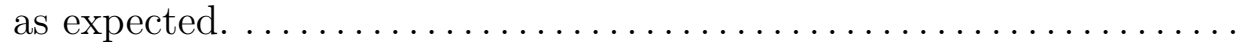

6.2 The enthalpy-based glide velocity model demonstrates a noticeable change in the stress response and in the mobile dislocation evolution with increasing temperature. The trend of these changes is not consistent across the range or temperatures nor consistent with expectations based on experimental observations: at the two higher temperatures, $225^{\circ} \mathrm{C}$ and $300^{\circ} \mathrm{C}$, the enthalpy-based velocity model predicts a non-physical increase in the strength of the material compared to the simulation at $150^{\circ} \mathrm{C}$, accompanied by a similar increase in mobile dislocation accumulation. $\ldots \ldots \ldots \ldots \ldots \ldots \ldots \ldots . . \ldots \ldots$

6.3 Variation of the stress response and mobile dislocation evolution, on an active slip system, to changes in the value of the power law velocity exponential constant, $m$, as given in Table 6.6, demonstrate a consistent reduction in both quantities with increasing values of

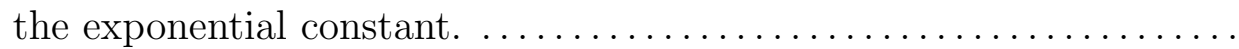


xviii

6.4 Changes in the stress response of $\alpha$ iron single crystals, loaded in [100] at $25^{\circ} \mathrm{C}$, with the set of exponential parameter variations given

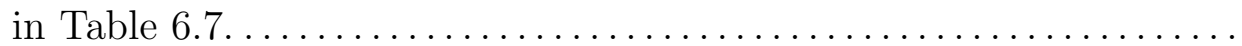

6.5 Evolution of the mobile dislocations on the active slip system $(21 \overline{1})[1 \overline{1} 1]$ in single crystal $\alpha$ iron, at $25^{\circ} \mathrm{C}$, with the exponential parameter sets

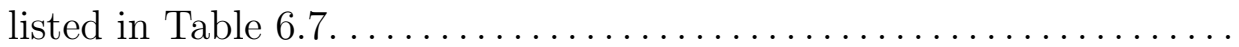

6.6 Changes in the stress response of $\alpha$ iron single crystals, loaded in [100] at $150^{\circ} \mathrm{C}$, with the set of exponential parameter variations given

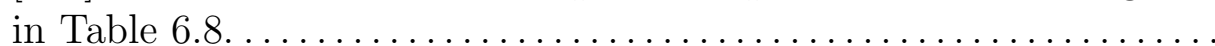

6.7 Evolution of the mobile dislocations on the active slip system (21) $[1 \overline{1} 1]$ in single crystal $\alpha$ iron, at $150^{\circ} \mathrm{C}$, with the exponential parameter

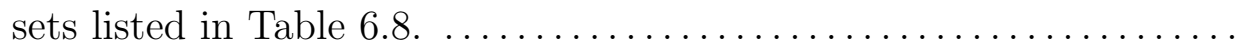




\section{LIST OF ACRONYMS}

BCC Body Center Cubic

CDD Continuum Dislocation Dynamics

DBTT Ductile-to-Brittle Transition Temperature

DD Dislocation Dynamics

FCC Face Center Cubic

FEM Finite Element Method

GND Geometrically Necessary Dislocations

IAEA International Atomic Energy Agency

INL Idaho National Laboratory

LRO Long Ranged Ordered

MD Molecular Dynamics

MOOSE Multiphysics Object Oriented Simulation Environment

RPV Reactor Pressure Vessel

SFTs Stacking Fault Tetrahedrons 
SIA Self-Interstitial Atom

TWIP Twinning-Induced Plasticity

VPSC Visco-Plastic Self Consistent 


\section{Dedication}

"'The Guide says that there is an art to flying,' said Ford, 'or rather a knack.'

'The knack lies in learning how to throw yourself at the ground and miss."'

- D. Adams, Life, the Universe, and Everything

To my parents, for their unwavering support, encouragement, prayers, and love, without which I may have never developed the knack required to complete this work or believed that I could.

Et à mon nounours, for the encouragement and emotional stability when I worried this work was crumbling around me, the pages of proofreading, and all of the hugs you have given me. Te amo.

And to my pony, Chaz, who spent countless hours listening about compiler and convergence issues when he really just wanted to eat apples and carrots. 


\section{CHAPTER 1. INTRODUCTION}

Nuclear power should play a prominent role in addressing the challenges of climate change. The dire consequences of failing to hold global warming temperatures below a $1.5^{\circ} \mathrm{C}$ increase from preindustrial levels were recently summarized in the UN Intergovernmental Panel on Climate Change report [71]. The Paris Agreement [126] emphasized the importance of drastically reducing carbon dioxide emissions along with other greenhouse gases to restrain the increase in global temperatures. During the 2015 U.N. climate talks, nuclear power was identified as having the ability to significantly contribute to efforts to combat climate change. Including and increasing nuclear power capability among the mix of energy generation methods makes attaining the tight decarbonization goals of $50 \mathrm{~g} \mathrm{CO} / \mathrm{kWh}$ set by the agreement more achievable [121].

Nuclear power plants, which supplied $20 \%$ of the energy generated in the United States of America in 2017, produce the majority of carbon emission free energy in the U.S. [134, 133]. Worldwide, nuclear power avoids the emission of nearly 2 billion tonnes of carbon dioxide per year [72]. Assuming a moderate growth rate of nuclear power plants through 2050, the 2018 International Atomic Energy Agency (IAEA) report predicts the reduction of an additional 1 billion metric tons of carbon dioxide emissions through the use of nuclear energy [72]. 
Additionally nuclear energy is the most reliable source of energy in the U.S. [192]; however, nuclear energy does face a number of challenges, from economic and policybased concerns to the technological aspects of power plant operation and waste storage [72]. On-going research in areas ranging from accident tolerant fuel to advanced reactor designs to evaluations of existing nuclear power plants under increasingly taxing potential transient and accident scenarios is aimed at addressing the technical challenges of nuclear energy.

In 2006 the Department of Energy's Basic Science Advisory Committee established the need for predictive multiscale material models under the extreme conditions in a nuclear reactor as a Scientific Grand Challenge [132]. In addition to the in-core components of a nuclear reactor, structural components of the nuclear power plant significantly influence the ability of a nuclear power plant to withstand potential transient conditions. Among these structural components is the Reactor Pressure Vessel (RPV) and the steam pipes used for the coolant loops [125].

In nuclear power plants the RPV is the containment vessel for the nuclear reaction. In addition to significant irradiation loads, the RPV is subjected to thermal and pressure loading from the the power plant operation. Long term irradiation doses of in-service RPVs increase the brittleness of the material due to the formation of irradiation defects. Understanding the role of the irradiation damage on the RPV is important both for relicensing efforts of the current fleet [6] and for devel- 
opment of advanced reactor designs [194]. Because of their ability to better resist irradiation-induced brittleness, ferritic/martensitic steels, which have a Body Center Cubic (BCC) crystal structure, have emerged as promising structural component candidates for advanced and next generation nuclear power plant designs [209]. In other advanced reactor designs, such as gas-cooled reactors, nickel-based alloys are being considered for use in the RPV structure [130]. Nickel-based alloys are already used in nuclear power plants for the coolant stream pipes, high chromium content nickel alloys, such as Alloy 625 and 690, show promise for use in the high temperature and high radiation environment [200]. Significant research is required, nonetheless, to develop these metals for the challenging radiation and thermal environment of the proposed GenIV operating conditions [23]. The mechanical response of these metals to the challenging environment is determined by how the microstructure evolves.

\subsection{Evolution of Microstructure under Irradiation}

The evolution of the microstructure of metals within a nuclear power plant RPV is intrinsically a multiphysics problem: atom displacements of the primary knockoff atom occur within picoseconds while refueling cycles generally span two years [194]. These nearly instantaneous atom displacements create significant changes in the structural material microstructure, and these changes limit how the structural 
components, such as the RPV, respond to the cyclic mechanical and thermal loading across refueling cycles. The microstructure changes from irradiation can also restrict the ability of the structural components to successfully withstand the extra loading caused by a potential transient event.

Nuclear reaction fission products interact with crystalline materials on the nanoscale by knocking single atoms out of place in the crystal lattice [196]. Over time, these displaced atoms and vacancies coalesce together to form vacancy clusters, precipitate clusters, and, in BCC materials, Self-Interstitial Atom (SIA) loops [167, 129]. In Face Center Cubic (FCC) materials, particularly those with low stacking fault energies, the SIA loops form Stacking Fault Tetrahedrons (SFTs) [69]. In this work, however, we focus on the role of thermal aging defects within a FCC material. Thermal aging occurs when a metal is held at a consistent temperature for a long duration, and the increased temperature enables the rearrangement of atoms within the lattice. Within nickel-based alloys precipitates often form as a result of thermal aging [184, 164].

Within the crystal lattice microstructure, when a dislocation encounters a defect it must either slip around or cut through the defect to continue moving [37]. These defects, caused by both radiation and thermal aging, interact with dislocations, increasing the brittleness of the material by impeding the movement of dislocations [210]. Similarly, thermally aged defects, such as precipitates, also impede dislocation motion by forcing the dislocation to either cut through the precipitate or to bow 
around it [89]. The impeded dislocation motion in the microstructure produces an increase in the yield stress and significant hardening in the plastic regime, causing the material to behave in a brittle manner at higher temperatures [209]. The physical interaction of the dislocations with these defects, and with other dislocations, governs how the the metal will respond to further loading and how it will continue to deform.

\subsubsection{Role of Modeling and Simulation}

Modeling and simulations are assuming an increasingly large role in understanding the mechanisms of irradiated material behavior and microstructure evolution[131]. Many researchers have attempted to establish modeling frameworks of the microstructure evolution of both structural metals and fuels under the radiation and thermal conditions of a nuclear power plant reactor core and pressure vessel, but challenges to these efforts persist, including the highly non-linear scale-dependent nature of irradiation processes $[17,116]$.

Multiscale modeling efforts mirror the expansive breadth of time and length scales involved in the physical process of structural metal irradiation. Atomic or Molecular Dynamics (MD) simulations span a few picoseconds and model individual atoms directly [14]. These simulations study the formation of irradiation defects [131] and the interactions among defects and dislocations [114]. On the opposite side of the 
spectrum, engineering scale simulations rely on heavily homogenous simplified models to study structures on the scale of meters over a time span of years [170].

To help bridge the gap between these two different types of simulations, many types of microstructure simulation tools have been developed. Dislocation Dynamics (DD) simulations explicitly model individual dislocations and the interactions of multiple dislocations with each other and with other lattice defects to develop dislocation evolution models $[204,8]$. This modeling technique, however, is best suited for the interior of a single material grain and not for modeling dislocation interactions with a grain boundary. Phase field [31, 84] and Visco-Plastic Self Consistent (VPSC) models [185, 98] both offer approaches which allow the simulation of polycrystalline materials. Phase field models are best suited to modeling the evolution of whole grains, including nucleation and absorption of grains, according to energy balances but are not currently suitable for modeling plastic hardening responses under mechanical loading. VPSC models, on the other hand, are adept at simulating the plastic response of polycrystals under mechanical loading in a computationally expedient manner; however, these models assume consistency in the individual grain shape. Crystal plasticity models, which calculate the evolution of dislocation and other crystal defect densities within a predefined polycrystalline geometry, address the need for a simulation tool for microstructure evolution under mechanical loading.

The crystal plasticity approach combines the ability to model complex polycrys- 
talline grain structures with mechanical loading. These mesoscale models can function as a bridge to connect microstructure evolution models to macroscale models of the Ductile-to-Brittle Transition Temperature (DBTT) curve and fracture. The toughness of a material, which governs the macroscale fracture behavior, is a function of both elastic deformation and plastic deformation. While the yield stress measurements capture the elastic contribution to a materials toughness, information about the dislocation movement is required to understand the plastic component of toughness. The ability of dislocations to move through a material depends on the types and densities of defects, including irradiation defects, present in the metal. Efforts to model the microstructure evolution at the mesoscale level have focused on writing constitutive equations for dislocation and radiation defect densities within a computational crystal plasticity framework [17, 28]. Recently developed crystal plasticity models, including the one presented in this work, retain the connection to the lower length scale dislocation density models by adopting the dislocation evolution relations from these studies $[161,101]$. As such, crystal plasticity models are a key tool to study the impact of irradiation and thermal aging on the microstructure evolution of nuclear power plant structural materials and therefore the resulting effect on the engineering scale. 


\subsection{Crystal Plasticity Models of Microstructure Evolution}

Crystal plasticity models play a critical role in capturing the effect of mesoscale changes in the microstructure on the engineering scale material properties. These continuum level models calculate plastic deformation by tracking dislocation movement and the interaction of these dislocations with other crystal defects, see Section 2.2.2, including other dislocations.

Crystal plasticity models track the evolution of microstructure by computing the growth of different dislocation and defect densities in response to mechanical deformation. Here will will first discuss dislocation glide mechanisms, including cross slip, before considering the influence of the grain boundaries on dislocation glide. We will continue with a discussion of deformation twinning followed by an overview of the mechanisms for dislocation movement resistance from other types of lattice defects, including those caused by irradiation.

\subsubsection{Dislocation Glide and Cross Slip}

The impact of dislocation evolution the plastic hardening response of a metal was proposed by Kocks and Mecking: these approaches treated dislcoation evolution as a two-term model with generation and annhilation terms [85, 117]. In 1983 Asaro formally connected the plastic slip, caused by dislocations, to the velocity and defor- 
mation gradients by defining the plastic velocity gradient to be the sum of the plastic slips on each of the slip systems [11]. The Orowan equation is used to relate the movement of dislocations to plastic slip. This equation states that the plastic slip rate is a function of the density of mobile dislocations, the Burgers vector of the full dislocation, and the average dislocation glide velocity [142].

\section{Dislocation Velocity Models}

Two different models for dislocation velocity are commonly applied in the crystal plasticity field: a power law model and an enthalpy-based model A dislocation glide driving force model, which includes a function of thermal activation energy, was first introduced by Kocks in 1976 to account for the impacts of thermal energy on the glide movements of dislocations [85]. The premise of this model is that dislocations at a higher temperature will have a higher velocity. Because of the direct dependence on temperature, this enthalpy-based relationship for dislocation velocity has been adopted by many research groups $[10,145,161]$. Around the same time Rice and Pierce et al. applied a straightforward power law model for the plastic slip due to dislocation motion $[159,146]$. This flow rule power law model was extended to a dislocation velocity model in which the exponent is applied to the ratio of the applied slip system shear stress to the slip system resistance [101]. This model, while not directly sensitive to changes in temperature, has the significant advantage of being computationally efficient while continuing to capture the experimental trends. 


\section{Dislocation Density Evolution Models}

Recent developments in crystal plasticity modeling have pushed towards dislocation density based models $[10,32]$. These models, developed for FCC metals, use dislocation density evolution equations with origins in the simple generation and annihilation proposed by Kocks [85]. These models separate out populations of screw and edge dislocations, yet the evolution equations for the dislocation populations still reduce to a binary multiplication and annihilation balance [10]. Roters et al. and Ma et al. , also working with FCC materials, use the concept of mobile and immobile dislocations instead in the dislocation evolution model [160] and [107]. Recent modeling of the BCC steels introduce a dislocation evolution model with terms for mobile nucleation, annihilation, and locking, where the mobile locking term acts as a source term for the immobile dislocation density $[145,28]$. While these models include a dynamic recovery term in the immobile dislocation evolution equation, this term is not coupled back to the mobile dislocation evolution equation. These two dislocation evolution models include terms for observable dislocation interaction mechanisms, including locking and cross slip. The implemented cross slip term, however, relies on an incomplete probability-based approach that can saturate the dislocation density and does not account for the stochastic nature of cross slip within the physical crystal microstructure. 


\subsubsection{Grain Boundary Influence}

The glide dislocation evolution models we have discussed thus far are developed for the interior of the metal grains, yet the boundaries between grains also play a significant role in the evolution of the microstructure. Near the grain boundaries the grains experience bending of the crystal lattice, and this lattice curvature can exert long-range forces on the grain in the region of the boundary. Long-range forces can be introduced into a computational crystal plasticity framework through a strain gradient term. Within crystal plasticity frameworks, the higher order strain terms have been tied to Ashby's Geometrically Necessary Dislocations (GND), which accommodate bending in the crystal lattice [12]. Nye's dislocation tensor is used to connect the strain gradient term to the GND density within a crystal. Kröner, in his earlier work, characterized the connection between dislocation density and Cosserat curvatures [33] with an incompatibility tensor [90]. The GND density is higher near the grain boundaries because of the localized lattice curvature; therefore GNDs can be used to incorporate the influence of grain boundaries on the evolution of dislocation density inside the grain.

\section{Geometrically Necessary Dislocation Models}

Physically based models rely on internal variables to model the evolution of microstructural features, such as dislocations and point defects. Dai and Parks, among 
others, used GND dislocation densities to incorporate work hardening into a crystal plasticity model by showing that the density of dislocations increases in areas of high strain gradient values [35]. Evers et.al. extended this modeling outlook to solve for the GND and glide dislocation densities on individual slip systems by including bi-crystal boundary regions within their model [46]; however, this a priori geometry forces a static choice of the reach of the grain boundary. In 2004 Mughrabi criticized many of these models for predicting overhardening of the glide slip system strengths when both glide dislocations and GNDs are directly included in the Taylor-type flow stress law proposed by Ashby [124]. As an alternate method of coupling the GNDs to the glide dislocation evolution, Ohashi introduces the concept of the mean free glide path. The mean free glide path is used to physically represent the increased glide slip system resistance from both forests of glide dislocations and GNDs [137, 138]. By including the density of the GNDs only in the calculation of the mean free glide path, the modified Bailey-Hirsch model avoids overhardening the crystal glide slip systems.

The calculation of the GND density is more substantial challenge. Although the Nye's dislocation density tensor cleanly connects the GND density to a strain gradient type term, there is not a universally accepted method of determining this strain gradient term. On the contrary, the breadth and variety of the theories and implementation attempts are a testament to the notrivial challenge of incorporating a physically based strain gradient term into crystal plasticity. The approaches can be 
broadly sorted into two different categories: $\mathrm{C}^{1}$ approaches and $\mathrm{C}^{0}$ formulations.

\section{$\mathrm{C}^{1}$ Formulations}

The idea to include more micromechanical aspects in continuum mechanics modeling is not new [186, 120], yet the challenges with selecting values for additional parameters and identifying the meaning of the boundary conditions are far from trivial. Mindlin's well-known micromechanics model, which has inspired many frameworks in the past five decades, has an additional 16 parameters, which must specified in addition to two elastic constants required for the classical continuum isotropic elasticity model. These challenges proved insurmountable and deterred the development of higher order continuum frameworks for a number of years.

In the past two decades, higher order continuum models have seen a resurgence for use in both plasticity and elasticity [2, 13]. Zbib and Aifantis first applied a higher order strain term, the Laplacian of the strain $\nabla^{2} \epsilon$, to smooth shear bands in a continuum plasticity model [66]. In 2001 Fleck and Hutchinson reformulated their iconic 1997 strain gradient theory which sought to extend isotropic $J^{2}$ plasticity by identifying invariants of the gradient of plastic strain rate [51]. The theory of Fleck and Hutchinson follows the work of Mindlin by using only the gradient of the strain as the higher-order term and introduced the long range term into the variation of virtual work equation. Inspired by the work by the Cosserat brothers [33], Forest et al. developed a micropolar theory which completely separates the additional rotation 
variables from the displacement variables except in the constitutive equations [52].

The introduction of the higher-order strain gradient into the governing equations requires the establishment of additional boundary conditions beyond the usual displacement and stress boundary conditions normally applied in continuum mechanics problems. Because of the nontrivial challenges in selecting appropriate boundary conditions, the most successful implementations of higher order $C^{1}$ micromechanics formulations are in the field of gradient elasticity. Most gradient elasticity studies have focused on 2D simulations [13]. Boundary conditions continue to be challenging, particularly for Hermite interpolation functions, and Askes suggests the use of couple stress traction free surfaces. Zervos et al. compare a $C^{1}$ formulation with a quadratic element with Hermite interpolation functions to a $C^{0}$ penalty method using quadratic elements. The Hermite $C^{1}$ formulations demonstrate quicker convergence with superior results [205]. Tangential higher order boundary conditions, that is mixed derivatives of the deformation gradients, are set to zero in $2 \mathrm{D}$ simulations [205]. Other research groups have developed new elements and interpolation functions to deal with the difficulties surrounding boundary conditions [205, 49].

\section{$\mathrm{C}^{0}$ Formulations}

The $\mathrm{C}^{0}$ formulations are constructed within the standard symmetric stress divergence governing equation and can be implements with a standard $\mathrm{C}^{0}$ element in a Finite Element Method (FEM) simulation. These approaches focus on methods to 
calculate a strain gradient term that depends on the deformation calculated in many neighboring elements. By averaging across the solution computed at many elements, $\mathrm{C}^{0}$ formulations can capture the influence of grain boundaries when the grain boundary runs through the element neighbor patch. Nonetheless, mesh dependence is a significant concern in these approaches.

Meissonnier et al. wrote a new User-defined element within ABAQUS to calculate the strain gradient term within a Hex27 element at the center quadrature point [118]; however, this approach continues to be mesh dependent because the strain term is not continuous across the elements in this modified $C^{0}$ element. Recently Abu AlRub et al. applied both the Laplacian of the strain and the gradient of the effective strain to model long-range effects within a crystal plasticity framework through a connection with Nye's dislocation tensor [1], claiming that the inclusion of both of these higher order strain terms is necessary for thermodynamic consistency. Abu Al-Rub implemented his higher order terms by using a meshless method to pull the strain values from the quadrature points within a capture radius and calculates the strain gradient value by fitting the selected strain gradient values. The process of calculating the fit strain gradient value may be computational expensive and the capture radius must be large enough to avoid the introduction of mesh dependence in the solution.

Other research groups use the gradient of the deformation gradient to capture the 
higher order strain measure. Kuroda and Tvergaard classify these theories as work conjugate and non work conjugate theories. Non work conjugate formulations do not require the use of high order stresses and connect the slip rates to back stresses arising from GND densities [93]. Arsenlis et al. developed physically-based model to capture non-homogeneous dislocation distributions by writing evolution equations for edge and screw dislocation fluxes which include a dependence on the gradients of the dislocation density [9]. In a similar non work-conjugate model, Evers et al. asserts that at a free surface the density of GNDs vanish [45]. Gurtin's microforce balance work is one of best-known work conjugate frameworks. The work conjugate theories, which are derived to be thermodynamically consistent, require additional boundary conditions to satisfy the higher order couple stresses introduced as work conjugates to the strain gradient terms [60]. Gurtin-type theories compute both edge and screw GND dislocations on all slip systems; these theories are computationally intensive and therefore have only been implemented thus far in 2D simulations [115], yet predictive models must be implemented in 3D to be applicable to many engineering problems.

Shizawa and Zbib examined the role of the dislocation density tensor and the incompatibility tensor within a complex crystal plasticity framework and concluded that only the dislocation density tensor, taken as the sum of the gradients of slip on each slip system, was necessary as an argument for thermodynamically consistent kinematic hardening [166]. 
A continued criticism of all strain gradient theories is the apparently arbitrary choice of length scale [103]. Recent works have attempted to address this criticism by employing the physical Burgers vector to calculate the GND density [36].

\subsubsection{Deformation Dislocation Twinning}

Several twinning mechanism models have been proposed for crystal plasticity and VPSC implementations. The first phenomenological models of twinning were introduced by [39] and implemented into a finite element crystal plasticity framework by Kalidindi $[75,76]$. Twinning models have been adapted to viscoplastic self consistent models for texture evolution $[185,18,19]$. Crystal plasticity models have been extended with twinning models for Twinning-Induced Plasticity (TWIP) steels, titianum, and nickel-based alloys among many other materials [161, 163, 77]. As in the case of glide dislocation evolution models, both phenomenological and physically based twinning models have been proposed.

Many of the crystal plasticity models for twins in nickel-based super alloys focus on the mechanism of microtwins. These models introduce the physically based concept of twin dislocation density which is related to the shear strain rate through the Orowan relation [191], an approach also taken in the modeling of $\epsilon$-martensite [197]. The microtwin mechanism relies on the presence of secondary $\gamma^{\prime}$ particles in com- 
merical alloys, which interact with Shockeley partials of glide dislocations to nucleate twins [86]. In certain spacing configurations, the secondary $\gamma^{\prime}$ particles enable the separation of the two Shockley partials to create a coherent stacking fault which can reconfigure into a true twin [191]. The spacing between lead and tail partial dislocations has been measured to be about 20-30 nm in a commercial alloy [89]. Given the critical role played by these $\gamma^{\prime}$ particles, the microtwinning crystal plasticity models explicitly include the density of secondary $\gamma^{\prime}$ particles $[169,77]$, although each model makes a simplifying assumption. Karthikeyan et al. assume that each twin dislocation has a fixed width and track the evolution of twin dislocations [77]. Song and McDowell assume a constant number of potential twins per volume in a rate independent formulation [169]. Nonetheless, neither of these models is appropriate for the binary $\mathrm{Ni}_{2} \mathrm{Cr}$ model alloy of interest to us in this work: the secondary $\gamma^{\prime}$ particles do not occur within the binary alloy.

\subsubsection{Strengthening due to Irradiation and Thermal Aging}

Because crystal plasticity models relate the accumulation of plastic slip to the movement of dislocations through the Orowan equation [142], the concept of slip system strength is used to compute the evolving hardening of each slip system. These

crystal plasticity models compute the slip system strength, or the resistance of each 
slip system to dislocation motion on that system, as a sum of the contributions to the slip system strength. Physically based frameworks write the constitutive slip system strength equations as functions of dislocation and defect densities within the crystal [135, 161]; both defects and other dislocations act as barriers to dislocation motion $[65,204]$. Each term in the sum of the strengths represents the stress exerted on the dislocations by a particular type of barrier: including the lattice friction for dislocation motion, the effect accumulated dislocations on the movement of mobile and twinning dislocations, and the irradiation and thermally aged defects as barriers to dislocation motion.

Irradiation damage has been incorporated into FCC crystal plasticity models through dispersed barrier models $[9,196]$; the radiation damage defect density is held as a constant. In 2004 Arsenlis et al. modified the dispersed barrier hardening model to use an empirical strength coefficient [9]. The number of defects can be related to the irradiation dose by fitting experimental data [35]. Newer crystal plasticity models focused on RPV applications couple irradiation defects to the dislocation evolution model by by adding to the slip resistance calculation [94, 145, 17, 28]. Of the irradiation defects occurring in BCC iron, the SIA loops are considered the primary defect contributing to low temperature irradiation hardening [167]; therefore, many of the irradiated BCC iron crystal plasticity models include evolution equations for the SIA loops $[17,28]$. Patra and McDowell have composed a crystal plasticity model 
to simulate the formation of these defect free channels [145]. These models rely on complex rank-2 tensor representations of the SIA loop densities and employ various means to restrict the interactions of the SIA loops with glide dislocations. This additional complexity in the model introduces additional unnecessary computational load to track the SIA loops on individual slip planes.

Thermally aged defects, such as long range ordered precipitates that occur in nickel-based alloys [164, 89], also act as barriers to glide dislocation motion. Often these defects are modeled with either the the dispersed barrier hardening model introduced for FCC irradiation defects or as antiphase boundary sheared particles [89]. In both cases, previous studies have assumed that theses defects are sheared by glide dislocations

\subsection{Identified Research Focus}

Among these varied crystal plasticity studies and implementations which we have discussed, none include all of the models to capture the interactions of the dislocations and defects relevant to nuclear industry structural materials. All of these models lack a consistent physical foundation within which to model these different aspects of microstructure evolution. There is a need for the development of a physically based unified crystal plasticity model capable of predicting the deformation behavior of 
multiple metallic materials based on the change of the material microstructure from radiation damage. A measured approach to developing a physically based crystal plasticity framework must include evaluation of models to ensure that the proposed terms capture the relevant physics in a reliable manner.

We present here a Continuum Dislocation Dynamics (CDD) crystal plasticity framework to capture the interaction mechanisms of multiple dislocations and defects within metals used in structural components of nuclear reactor power plants. This crystal plasticity framework consists of dislocation evolution equations informed by physical interaction mechanisms. We have selected physically based models to expand the crystal plasticity framework with a temperance informed by the end use application material and environment. In this work we have focused on two simpler metals, BCC $\alpha$ iron and the FCC binary $\mathrm{Ni}_{2} \mathrm{Cr}$ alloy, to focus on capturing the dislocation interaction mechanisms with with other dislocations and defects. The use of our crystal plasticity framework to successfully model two different nuclear power plant structural materials demonstrates the strength of our measured approach.

This physically based dislocation density crystal plasticity model will be implemented into the Multiphysics Object Oriented Simulation Environment (MOOSE), which was developed by Idaho National Laboratory (INL) and is currently used to conduct predictive simulations of nuclear reactor fuels [195]. As a component of the MOOSE framework, the predictive CDD crystal plasticity models proposed here can 
play a role in advancing a sustainable power source that can contribute to mitigating climate change. The implementation of this crystal plasticity model within the open source MOOSE code developed and maintained by the leading nuclear national laboratory ensures the continued maintenance and use of the model within the nuclear research domain.

\subsection{Overview of Thesis}

The organization of the remainder of this thesis is as follows. We begin with an overview of the theory governing continuum mechanics in Chapter 2. Preceding the discussion of crystal plasticity theory, we present a brief introduction of the crystalline structure of metals. This chapter is completed with a summary of the numerical implementation of the CDD crystal plasticity framework within the MOOSE software.

In Chapters 3, 4, 5, and 6, we present the individual models and the numerical implementation of the different functionalities of the developed CDD crystal plasticity model to address the needs identified above. Chapter 3 focuses on the modeling of $\mathrm{BCC} \alpha$ iron for use in a nuclear reactor pressure vessel application, including the introduction of a stochastic cross slip model. The results presented in this chapter also involve the effect of irradiation defect hardening on the glide movement of dislocations in the $\alpha$ iron crystals. In Chapter 4 we demonstration the use of a nodal 
patch recovery algorithm to calculate a semi-continuous measure of the plastic velocity gradient and the calculation of geometrically necessary dislocations from this recovered tensor. The focus of Chapter 5 is the implementation of a deformation twinning mechanism, including the extension of the CDD framework to allow multiple contributions from different dislocation movement systems to the plastic slip tensor. The results presented in this chapter are compared to a set of micropillar compression tests, which include unaged and aged samples with long-range precipitates present. Chapter 6 consists of a brief comparison of the two dislocation velocity models previously introduced in Section 1.2.1. The suitability of the two models for different simulation criteria is discussed.

In the conclusions, Chapter 7, we summarize the impact of this thesis work along with recommendations for future work. These recommendations address both the need to increase the utility of microstructure evolution research to engineering scale simulations and the exciting possibilities of expanding the study of microstructure evolution with additional modeling techniques. The work presented in this thesis is a foundational step to achieving both of these seemingly divergent goals within the MOOSE software environment. 


\section{CHAPTER 2. THEORETICAL OVERVIEW AND NUMERICAL IMPLEMENTATION}

Solid mechanics is the study of how a solid body changes shape in response to applied loads and constraints. These loads can have wide variety of forms, yet in this work we will focus on mechanical and thermal loads. As an example, consider a solid soft material. Applying a load to this material results in a shape change of the material. Over time as a load continues to be applied, the type of the body's shape change response differs as the characteristic properties of the body evolve. Solid mechanics, a branch of continuum mechanics, provides the mathematical tools to understand how an applied load produces a shape change. The applied load creates a stress in the material, that stress is correlated with a change in strain, and the strain produces a change in the material body shape. We term this shape change deformation.

Using the tools of solid mechanics, we can represent the relationship between displacement and strain with kinematic relations; these relations map changes in the position of all points in the material from the original shape to the current deformed shape. Constitutive equations describe the relationship between strain and stress; while these equations have similar forms, they depend on the specific properties of each material studied. The relationship between the stress experienced by a material 
as a result of an applied load is explained with the Cauchy equations of motion.

Within classical continuum mechanics, the studied solid body is treated as an idealized collection of continuously distributed particles: a continuum [82]. Nonetheless, many physical solid bodies can be sorted into smaller components. The relationships explaining the interaction of these smaller components can be necessary to more fully describe how a solid body deforms in response to an applied load. Metals are examples of materials which have been studied extensively by separating the homogeneous continuum body into smaller components based on physical observations [69]. Among the many fields which study the mechanics of crystalline metals, crystal plasticity has been established as a capable tool to explore the relationship between crystalline microstructure evolution and engineering scale homogeneous stress response $[11,116,161]$.

Physically-based crystal plasticity models, including the Continuum Dislocation Density (CDD) crystal plasticity framework which is the focus of this work, calculate the evolution of dislocation densities under stress [10, 161, 101]. The dislocation evolution is further coupled to the densities of additional irradiation defects. Densities are used to track dislocations and irradiation defects because crystal plasticity is a continuum level mathematical model. The formulation within a continuum mechanics framework enables crystal plasticity models to simulate longer time scales and larger length scales than atomistic and dislocations dynamics models which explicitly track 
each individual dislocation and defect. The common foundation in continuum mechanics between crystal plasticity and engineering scale models allows for more direct transfer of mesoscale material behavior prediction to inform engineering scale models. The work presented in this thesis adds to the field of crystal plasticity through the development and implementation of physically based models for metals in irradiation conditions common for in-service nuclear reactor pressure vessel parts.

We begin our discussion of the theory germane to this works with a focus on the fundamental solid mechanics concepts within an elastic homogeneous body before focusing on the concepts specific to crystalline metals. After a brief overview of the physical structure of cubic crystalline metals and common crystal microstructure components, we will describe the complex constitutive crystal plasticity relationships used to calculate the stress resulting from the evolution of crystal dislocations and defects. We conclude this chapter with a synopsis of the implementation of the crystal plasticity constitutive equations in the MOOSE and Grizzly software codes.

\subsection{Continuum Solid Mechanics Overview}

Within this document, a scalar is indicated by a lower-case italicized letter, $a$, a vector is indicated by a lower-case bold letter, $\mathbf{v}$, and a Rank-two tensor is indicated by an upper-case bold letter, $\mathbf{T}$. We indicate a Rank-four tensor with an outlined 
bold upper-case letter, $\mathbb{J}$. In Einstein notation the number of indices indicates the rank of the tensor: a scalar with a rank of 0 is indicated by $a$, a Rank-one vector is written as $v_{i}$, a Rank-two tensor is denoted with the form $T_{i j}$, a Rank-three tensor is indicated by three indices, $A_{i j k}$, and a Rank-four tensor is written with four indices, $J_{i j k l}$. The double dot product is given as the inner dot product, $T_{i j}: S j i$, and the triple dot product is specified as $A_{i j k}: \cdot B_{k j i}$ except where otherwise explicitly noted.

\subsubsection{Deformation and Kinematic Equations}

The movement, or change in position, of a material point within a solid continuum body from a position in the original configuration to a new position is termed displacement. Deformation is a function of displacement and is the change in position of material points within the continuum body, with respect to the original position of the body. The original body position for each material point is defined within a fixed reference coordinate system, generally taken at time $t=0$. By convention, this position in the reference configuration is denoted with a capital letter [111], e.g. $X$ as shown in Figure 2.1 for point $P$. All of the points in the body at this time frame are considered to be in the reference, or Lagrangian, configuration [82]. After a load has been applied to the solid body such that its shape changes, the new position of the same point is defined with the current coordinate configuration as the current 


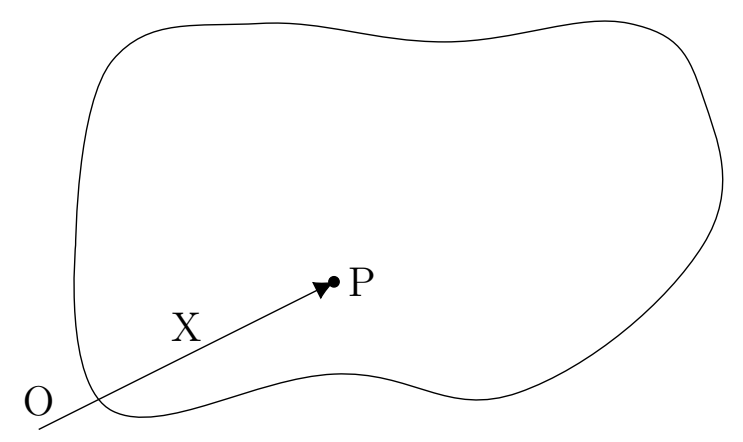

Figure 2.1: Example of a deformed continuum solid body with the position of a point shown in the reference configuration.

position vector $\mathbf{x}[156]$ :

$$
\mathbf{x}=x(\mathbf{X}, t)
$$

where $t$ is the time. We note directly in Eq (2.1) that the current position is a function of the reference position. In an analogous manner to the reference configuration, the current configuration is defined as the positions of the set of material points within the body at the current time $t$. This configuration is also termed the Eulerian configuration [22].

The assumption of smooth continuous movement between the two positions, formalized as the axiom of continuity [82], allows us to write the inverse of Eq (2.1) to find the reference configuration position as a function of the current position:

$$
\mathbf{X}=X(\mathbf{x}, t)
$$

where $t$ is the time as in Eq (2.1). The relationship between these two positions is 
defined with a displacement vector $u$

$$
\begin{aligned}
& u(\mathbf{X}, t)=x(\mathbf{X}, t)-\mathbf{X} \\
& u(\mathbf{x}, t)=\mathbf{x}-X(\mathbf{x}, t)
\end{aligned}
$$

where the first displacement vector, $\mathrm{Eq}$ (2.3), is defined in the Lagrangian configuration and the latter displacement vector, Eq (2.4), is defined in the Eulerian configuration. The choice of which configuration to use is heavily influenced the constitutive relation formulation used, Section 2.1.3.

The rate of displacement change is termed the velocity. As with displacement, the velocity can be defined with respect to either the Lagrangian or the current (Eulerian) configuration:

$$
\begin{aligned}
& v=v(\mathbf{X}, t)=\frac{d \mathbf{u}(\mathbf{X})}{d t} \\
& v=v(\mathbf{x}, t)=\frac{d \mathbf{u}(\mathbf{x})}{d t}
\end{aligned}
$$

The choice to define the velocity vector in the current configuration, Eq (2.6), has complicating implications for the calculation of the acceleration with the material derivative [82]. The reference configuration definition, Eq (2.5), leads to a straightforward calculation of the acceleration because the Lagrangian material derivatives are constant [111]. 


\section{Deformation Gradient Tensor}

With the mathematical description of the shape change of a body, we now require a method to describe how a solid body deforms. In order to accomplish this task, we consider each point in the solid body not as independent unconnected points but rather as so-termed line elements. In the reference configuration we designate the line element vector as $d \mathbf{X}$ and in the current configuration the line element vector is labeled as $d \mathbf{x}$. Note the correspondence of the notation used for line elements and

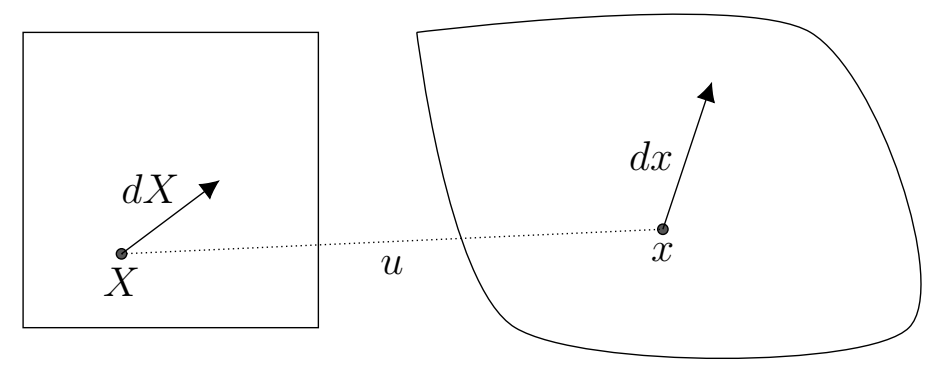

Figure 2.2: Example of a continuum solid body in both the reference and current configurations with the line elements labeled.

that used for the material point in the deformation definitions, Eqs (2.1) and (2.2).

The deformation gradient provides a mathematical concept of the rate of change of deformation and relates the line element $d \mathbf{x}$ in the current configuration to the line element $d \mathbf{X}$ in the reference configuration[111].

$$
d \mathbf{x}_{i}=\frac{\partial x_{i}}{\partial X_{K}} \cdot d X_{K}=\nabla_{k} x_{i} \cdot d X_{K}=F_{i K} d X_{K}
$$


where we have applied index notation, subscripts $i$ and $K$ to designate the line element vectors in the current and reference configurations, respectively. From Eq (2.7) and using the definition of displacement in the current configuration, Eq (2.4), we can define the rank-2 tensor deformation gradient, $\mathbf{F}$ as

$$
F_{i K}=\frac{\partial x_{i}}{\partial X_{K}}=\delta_{i K}+u_{i, K}
$$

where the last term $u_{i, K}$ designates the derivative of the displacement in the current configuration with respect to the reference configuration [82].

The deformation gradient allows us to find the deformation in the reference configuration as

$$
X_{K, i}=F_{i K}^{-1}=\frac{\partial X_{K}}{\partial x_{i}}=\delta_{K i}+u_{K, i}
$$

where we have applied Eq (2.3) for the definition of the displacement. The inversion of the deformation gradient is always possible due to the axiom of continuity [82]. This axiom takes the mathematical form

$$
\left|\frac{\partial x_{i}}{\partial X_{K}}\right| \neq 0
$$

from which the obvious extension is

$$
\operatorname{det}\left(F_{i K}\right)=J \neq 0
$$

where $J$ is termed the Jacobian.

We recognize that a deformation can be generalized as a combination of a linear stretching motion and a rotation movement. The stretching movement of a material 
point from its original position occurs as a translation [111] while rotation describes an angular change of position, usually with respect to the coordinate system origin [22]. These two motions are intertwined, however, in such a fashion that we cannot decouple them with an additive superposition relationship. The deformation gradient can be multiplicatively decomposed via the Cauchy theorem for nonsingular tensors into two rank-2 tensors, one representing the stretch and the other rotation motion.

$$
\mathbf{F}=\mathbf{R} \cdot \mathbf{U}=\mathbf{V} \cdot \mathbf{R}
$$

where $\mathbf{R}$ is the orthogonal rotation tensor and $\mathbf{U}$ and $\mathbf{V}$ are the symmetric right and left stretch tensors, respectively [156]. We will return to this concept of stretch and rotation tensors after discussing the strain measures, Section 2.1.2, to further describe deformation.

\subsubsection{Strain Measures}

The concept of strain is used to describe the rate of displacement, that is, the relative change in the displacement of the body. The rate of displacement of a solid body has a significant impact on the solid's stress response, ranging from the rapid brittle fraction seen in explosions to the slow creep of glass windows over centuries. Introduced to students first as a linear measure of change in length over the original length [156], formally the rank-2 strain tensors are defined with the square of the line 
element length change. The squared line element length is given as

$$
\begin{gathered}
d S^{2}=d \mathbf{X} \cdot d \mathbf{X} \\
d s^{2}=d \mathbf{x} \cdot d \mathbf{x}
\end{gathered}
$$

in either the reference configuration, Eq (2.13), or the current configuration, Eq (2.14). The strain tensor, in the Lagrangian configuration, is defined as

$$
d s^{2}-d S^{2}=2 d \mathbf{X} \cdot \mathbf{E} \cdot d \mathbf{X}
$$

where $\mathbf{E}$ is the Lagrangian strain tensor [111].

The squares of the line elements can also be related to the deformation gradient tensor, in the reference configuration

$$
\begin{aligned}
d S^{2} & =d \mathbf{X} \cdot d \mathbf{X}=\left(\mathbf{F}^{-1} \cdot d \mathbf{x}\right) \cdot\left(\mathbf{F}^{-1} \cdot d \mathbf{x}\right) \\
& =d \mathbf{x} \cdot\left(\mathbf{F}^{-1}\right)^{T} \cdot\left(\mathbf{F}^{-1}\right) \cdot d \mathbf{x}
\end{aligned}
$$

and in the current configuration

$$
\begin{aligned}
d s^{2} & =d \mathbf{x} \cdot d \mathbf{x}=(\mathbf{F} \cdot d \mathbf{X}) \cdot(\mathbf{F} \cdot d \mathbf{X}) \\
& =d \mathbf{X} \cdot \mathbf{F}^{T} \cdot \mathbf{F} \cdot d \mathbf{X}
\end{aligned}
$$

Noting the similarities between the formal definition of strain, Eq (2.15) and the expressions for the squared line elements as a function of the deformation gradients, Eqs (2.16) and (2.16), [26] and [58] introduced the following deformation tensors

$$
\begin{aligned}
\mathbf{B}^{-1} & =\left(\mathbf{F}^{-1}\right)^{T} \cdot \mathbf{F}^{-1} \\
\mathbf{C} & =\mathbf{F}^{T} \cdot \mathbf{F}
\end{aligned}
$$


where $\mathbf{B}$ and $\mathbf{C}$ are the left and right Cauchy-Green deformation tensors, respectively [187]. The left and right Cauchy-Green deformation tensors can be used to find the squares of the line element lengths by substituting Eqs (2.18) and (2.19) into Eqs (2.16) and (2.17).

The Cauchy-Green deformation tensors enable the introduction of the Green-Saint Venant and the Almansi-Hemel strain tensors. From the definitions of the strain tensor in Eq (2.15) and the right Cauchy-Green deformation tensor in Eq (2.19), we can write

$$
\begin{aligned}
d s^{2}-d S^{2} & =d \mathbf{X} \cdot \mathbf{C} \cdot d \mathbf{X}-d \mathbf{X} d \mathbf{X} \\
& =d \mathbf{X} \cdot(\mathbf{C}-\mathbf{I}) \cdot d \mathbf{X} \\
& =2 d \mathbf{X} \cdot \mathbf{E} \cdot d \mathbf{X}
\end{aligned}
$$

such that the Lagrangian Green-Saint Venant strain tensor can be expressed as a function of the right Cauchy-Green deformation tensor [168]

$$
\mathbf{E}=\frac{1}{2}(\mathbf{C}-\mathbf{I})
$$

where $\mathbf{I}$ is the rank-2 identity tensor. In a similar fashion we can express the Eulerian Almansi-Hemel strain tensor as a function of the left Cauchy-Green deformation tensor.

$$
\begin{aligned}
2 d \mathbf{x} \cdot \epsilon \cdot d \mathbf{x} & =d s^{2}-d S^{2} \\
& =d \mathbf{x} d \mathbf{x}-d \mathbf{x} \cdot \mathbf{B}^{-1} \cdot d \mathbf{x} \\
\epsilon & =\frac{1}{2}\left(\mathbf{I}-\mathbf{B}^{-1}\right)
\end{aligned}
$$


We note that by definition the Green-Saint Venant and Almansi-Hemel strain tensors are symmetric from the multiplication of the deformation gradient tensors within the right and left Cauchy-Green deformation tensor definitions.

The Lagrangian strain tensor, Eq (2.21), enables us to calculate the strain with respect to the reference configuration as a function of the deformation gradient. This capability is necessary for the crystal plasticity formulation we will discussion in Section 2.3.

\section{Small Strain Definition}

In passing we mention the specialized case of small strain. Expressing the Lagrangian strain tensor, Eq (2.21), as a function of the deformation gradient and applying the definition of the deformation gradient as a function of displacement, Eq (2.8), we arrive at the expression for strain [82]

$$
\begin{aligned}
E_{K L} & =\frac{1}{2}\left(C_{K L}-I_{K L}\right)=\frac{1}{2}\left(F_{K i}^{T} F_{i L}-I_{K L}\right) \\
& =\frac{1}{2}\left[\left(\delta_{i K}+u_{i, K}\right)\left(\delta_{i L}+u_{i, L}\right)-\delta_{K L}\right] \\
& =\frac{1}{2}\left[u_{K, L}+u_{L, K}+u_{m, K} u_{m, L}\right]
\end{aligned}
$$

The small strain assumption applied in this case is

$$
\left|u_{i, K}\right|<<1
$$


such that the squared term in Eq (2.24) is negligible [22]. The conventional small strain tensor is given as [168]:

$$
\mathbf{E}=\frac{1}{2}(\mathbf{u} \nabla+\nabla \mathbf{u})
$$

Additionally in the case of small strain we assume that no rotation occurs as part of the deformation. Mathematically we express this assumption by setting the rotation tensor from Eq (2.12) equal to the rank-2 identity tensor.

\section{Velocity Gradient}

As we have defined a rate of displacement tensor, we can also define a rate tensor for the deformation gradient. This deformation gradient rate tensor is termed the velocity gradient. The definition of the velocity gradient relies on the concept of the material derivative. Also termed the spatial gradient of velocity [111]

$$
\mathbf{L}=\operatorname{grad} \mathbf{v}
$$

the velocity gradient is related to the material derivative of the deformation gradient $[82]$

$$
\dot{\mathbf{F}}=\mathbf{L} \cdot \mathbf{F}
$$

Similar to the deformation gradient, the velocity gradient may be decomposed into a stretching rate tensor, $\mathbf{D}$ and the spin tensor, $\mathbf{W}$ as

$$
\mathbf{L}=\mathbf{D}+\mathbf{W}
$$


where $\mathbf{D}$ is symmetric and $\mathbf{W}$ is skew-symmetric [111]. In the crystal plasticity formulation, which we will introduce in Section 2.3, we will deal directly with the velocity gradient tensor.

With the deformation gradient, strain, and velocity gradient tensors defined to describe the relative shape change of the solid body, we proceed to discuss the connection between stress and strain know as the constitutive equations.

\subsubsection{Elastic Constitutive Equations}

Constitutive equations are mathematical relationships to connect the stress and strain behavior of a material. Ideally based in the physics of the material under study, these equations range from simplified linear and fully recoverable, or elastic, responses to complex nonlinear models for viscoelasticity and plasticity [168]. Constitutive equations cannot represent the individual atom behaviors within a continuum formulation and are therefore descriptions of an average for a statistically uniform material [22]. For this reason the crystal plasticity constitutive equations we will discuss in Section 2.3 are cast in terms of dislocation densities instead of individual dislocations. In this section, to illustrate the constraints on all constitutive equations, we will focus on the well-known generalized Hooke's Law elastic constitutive model. 
Constitutive equations must follow the requirements [22]:

1. Obey the restrictions imposed by the first and second laws of thermodynamics,

2. Satisfy the condition of objectivity.

After discussing the restrictions placed on constitutive equations by these laws of thermodynamics, we will introduce the the objectivity requirement on the stress tensor produced by the constitutive equation. We will conclude the chapter with a discussion of the stress power concept for a linearized elastic general Hooke's law constitutive equation. A discussion of the material symmetries which reduce the number of independent constants in this constitutive elasticity tensor will follow. In this section we will make use of two stress measures, $\mathbf{T}$, the Cauchy stress on the current configuration and $\mathbf{S}$, the second Piola-Kirchhoff stress on the reference configuration. These two rank-2 stress measures will be formally introduced in Section 2.1.4, including the symmetric nature which we will employ in this section.

\section{Restrictions from the Laws of Thermodynamics}

The first law of thermodynamics states the conservation of energy; that is, the total energy of a system must equal the input power and the heat input rate [111]. In continuum mechanics the power input is the rate at which external applied loads, tractions, and body forces do work on the body. We will utilize this concept in our discussion on internal stress power, Section 2.1.3. Formally, the nonpolar energy 
balance equation implied by the first law of thermodynamics is

$$
\rho \frac{u}{t}=T_{i j} D_{i j}+\rho r-\frac{\partial q_{j}}{\partial x_{j}}
$$

where $\mathbf{u}$ is the specific internal energy, $\mathbf{T}$ is the Cauchy stress measure which is related to surface tractions as $t_{j}=T_{j i} n_{i}, \mathbf{D}$ is the stretching rate tensor from Eq (2.29), $\rho$ is the material density, $r$ is the internal distributed heat source, and $\mathbf{q}$ is the heat flux [111]. The second law of thermodynamics states that entropy cannot be reduced: for a reversible process, such as elastic deformation, the entropy is constant, and in an irreversible process, such as plastic deformation, the entropy may only increase [21]. The Clausius-Duhem inequality recasts the second law of thermodynamics for continuum mechanics

$$
\frac{d s}{d t} \geq \frac{r}{\theta}-\frac{1}{\rho} \nabla \cdot \frac{\mathbf{q}}{\theta}
$$

where $s$ is the entropy and $\theta$ is the temperature [111]. With some rearranging and using Eq (2.30) the inequality can be expressed as a function of the stress measure and the stretching tensor

$$
\mathbf{T}: \mathbf{D}-\rho\left(\frac{\partial u}{d t}-\theta \frac{d s}{d t}\right) \geq 0
$$

Using the concept of Helmholtz free energy

$$
\Psi=u-\theta s
$$


the Clausius-Duhem inequality becomes [22]

$$
\mathbf{T}: \mathbf{D}-\rho\left(\frac{\partial \Psi}{d t}-s \frac{\partial \theta}{\partial t}\right) \geq 0
$$

This form of the inequality can provide a more straightforward path to determine if a constitutive equation obeys the restrictions from the second law of thermodynamics.

\section{Stress Objectivity Constraints}

The second requirement for a constitutive equation is that the calculated stress measure does not change as the material reference frame changes, that is, the stress tensor must be material frame-indifferent, or objective, measures. To be frame indifferent, a second order tensor must transform under changes of reference frame:

$$
\mathbf{A}^{*}=\mathbf{Q} \cdot \mathbf{A} \cdot \mathbf{Q}^{T}
$$

where $\mathbf{Q}$ is a proper orthogonal tensor which characterizes the relative rotations between frames of reference [168]. For a constitutive equation relating the Cauchy stress, $\mathbf{T}$, to the deformation gradient,

$$
\mathbf{T}=f(\mathbf{F})
$$

to be considered material frame-indifferent it must satisfy

$$
f(\mathbf{Q} \cdot \mathbf{F})=\mathbf{Q} \cdot f(\mathbf{F}) \cdot \mathbf{Q}^{T}=\mathbf{Q} \cdot \mathbf{T} \cdot \mathbf{Q}^{T}
$$

for all proper orthogonal Q [168], where the objective stress measure tensor is

$$
\mathbf{T}^{*}=\mathbf{Q} \cdot \mathbf{T} \cdot \mathbf{Q}^{T}
$$




\section{Elastic Stiffness Tensor}

The generalized Hooke's law for elastic materials is perhaps the best-known example of a constitutive law. Describing a fully reversible process, this constitutive law satisfies the thermodynamic requirements by exactly conserving entropy, see Eq (2.34), by assuming the stress tensor is a linear function of strain

$$
T_{i j}=C_{i j k l} \epsilon_{k l}
$$

where $\mathbf{C}$ is known as the rank-4 stiffness tensor [156]. Note that we have employed the linearized small strain in the current configuration, $\epsilon$, here, which is symmetric by definition similar to the linearized Lagrangian strain, Eq (2.26). Exploiting the symmetries of the stress and strain tensors, the number of independent components of the stiffness tensor can be reduced:

$$
\begin{gathered}
T_{i j}=T_{j i} \Rightarrow C_{i j k l}=C_{j i k l} \\
\epsilon_{k l}=\epsilon_{l k} \Rightarrow C_{i j k l}=C_{i j l k}
\end{gathered}
$$

The symmetry in the strain tensor means that the skew-symmetric component of the stiffness tensor is arbitrary [168]. These symmetries, Eq (2.40), reduce the number of independent stiffness tensor components from 81 to 36 . We further reduce the number of independent stiffness tensor components with the concept of strain energy density. The strain energy density for a hyperelastic material is defined as[168]:

$$
U(\epsilon)=\int_{0}^{\epsilon} \mathbf{T}: d \epsilon \Rightarrow T_{i j}=\frac{\partial U(\epsilon)}{\partial \epsilon_{i j}}
$$


which is path independent and related to the Helmholtz free energy we introduced in Eq (2.33). Differentiating the strain energy density expression yields

$$
\frac{\partial^{2} U(\epsilon)}{\partial \epsilon_{i j} \epsilon_{k l}}=\frac{\partial^{2} U(\epsilon)}{\partial \epsilon_{k l} \epsilon_{i j}}=C_{i j k l}
$$

Since the order of differential is arbitrary, the stiffness tensor must have symmetry in the first and last pairs of indices:

$$
C_{i j k l}=C_{k l i j}
$$

which gives only 21 independent tensor components [111, 168, 156].

Many materials, including the cubic crystals which are the subject of this work, exhibit additional symmetries; for these materials the stiffness tensor is further simplified. Cubic crystal materials, including iron and nickel, are orthotropic. The three orthogonal planes of symmetry decouple the shear stress, that is the nondiagonal components of the stress tensor, from the normal strain components, which lie on the diagonal, such that only 9 components of the stiffness tensor are independent [168]. In the elastic-plastic constitutive relationships we will apply in the modeling of the crystals, Section 2.3, we will use this orthotropic material symmetry stiffness tensor.

\section{Work Conjugate Pairs and Stress Power}

In order to write a constitutive equation, we must ensure that we are relating the appropriate strain and stress measures. The concept of work done on a body by a stress measure $[168,1]$, provides a method to determine appropriate stress and strain 
measures through work conjugate pairs [22]. Oftentimes the rate of work done by the stress is expressed with the Cauchy stress measure $\mathbf{T}$ and the stretch rate tensor $\mathbf{D}$ which we have used previously in Section 2.1.3.

Here we wish to determine the work conjugate to the Lagrangian strain rate tensor, $\dot{\mathbf{E}}$, for use in writing constitutive equations on the reference configuration as is done in our crystal plasticity formulation, Section 2.3. From the definition of stress power given by [111] or the work done by external forces [168]

$$
P_{\text {int }}=\int_{A} t_{i} v_{i} d A+\int_{V} \rho b_{i} v_{i} d V
$$

where $\mathbf{t}$ is the traction associated with the Cauchy stress measure and $\mathbf{b}$ is a body force. We can write this integral on the underformed configuration, introducing the second Piola-Kirchhoff stress measure, S. Through the application of the equations of motion and the symmetry of the second Piola-Kirchhoff stress measure, discussed in Section 2.1.4, we arrive at the expression

$$
\int_{V_{o}} S_{i K} \dot{E}_{i K} d V_{o}+\int_{V_{o}} \frac{1}{2} v_{i} v_{i} d V_{o}=0
$$

which demonstrates that the second Piola-Kirchhoff stress and the Lagrangian strain rate tensors are work conjugates. Thus, we can write our crystal plasticity constitutive relationships using these two measures. 


\subsubsection{Stress Measures and Equations of Motion}

The Rank-2 tensor stress measures are used within continuum mechanics to characterize the internal forces acting on a body at a material point. In order to describe these internal forces we must first examine the external forces acting on the body before defining the stress measures. Once defined, the form of the stress tensors is restricted by the equations of motion. The equations of motion also provide the governing partial differential equation which we will solve with a finite element method simulation, Section 2.4.

The external forces acting on a body can be classified into two categories: body forces and surface forces [111]. Body forces are long-range action or loads applied to every particle in the solid body, such as gravity [156]. Surface forces, on the other hand, act only on a portion of the material points which lie on the body's surface, such as contact forces [168]. A surface force per unit area is termed a traction [111]. The mathematical representation of a body force, $\mathbf{b}$, acting on a deformed body in the current configuration is

$$
B=\int_{V} \rho \mathbf{b} d V
$$

and the mathematical representation of a surface force acting on the deformed body surface is

$$
T=\int_{S} \mathbf{t} d a
$$


where $\mathbf{t}$ is the traction vector [111]. Tractions can be characterized by the direction in which they interact with the surface. The surface at a material point can be described by $\hat{\mathbf{n}}$, the unit outward normal at the point [168]. A traction, or the component of the traction, which act on the surface parallel to this unit normal vector is termed the normal traction. The other components, which act perpendicular to the unit normal vector, are termed shear tractions. We will make use of shear forces acting along a surface in the formulation of the crystal plasticity constitutive model, which we will discuss in Section 2.3.

The Euler-Cauchy stress principle [82] states that tractions exist across every internal surface of a body and that the equations of motion, Section 2.1.4, apply both to the whole body and any interior surface [82]. These internal tractions are defined on an infinitesimal internal surface element, $\Delta a$, which also has a unit normal vector, $\hat{\mathbf{n}}$. Employing Newton's third law, which implies that a traction vector acting on one side of a material point is equal and opposite to the traction vector acting on the other side of the surface element, we introduce the idea of the Cauchy tetrahedron in the current configuration [168]. About a single material point we draw a tetrahedron whose sides and base align with the planes of the coordinate system and whose fourth face does not pass through the material point of interest. The traction vector normal to this fourth surface can be represented as the sum of the tractions acting along the unit normal vectors of the base and sides. Taking the limit of the tetrahedron until 
the tetrahedron collapses to the point such that we can express the traction as [26]

$$
\mathbf{t}=t_{1} \hat{e}_{1}+t_{2} \hat{e}_{2}+t_{3} \hat{e}_{3}
$$

where $\hat{e}_{i}$ are the unit vectors of the coordinate system. With the Euler-Cauchy stress principle, we define the Cauchy stress as [187]

$$
t_{i}=n_{j} T_{i j}
$$

where $\mathbf{T}$ is the Cauchy stress, defined on the current configuration which we utilized in Section 2.1.3. Similar to the convention used for the tractions, the normal components of the Cauchy stress tensor are those which lie along the diagonal of the tensor and the shear components are the non-diagonal components.

\section{Second Piola-Kirchhoff Stress}

As we saw in the discussion of Constitutive Equations we find it useful to define a stress measure with respect to the reference configuration. Both the first and second Piola-Kirchhoff stress measures are defined on the reference configuration, although only the second Piola-Kirchhoff stress tensor is symmetric [82].

The second Piola-Kirchhoff stress tensor, S, can be defined from the Cauchy stress tensor with the help of the deformation gradient [111] as

$$
\mathbf{S}=J \cdot \mathbf{F}^{-1} \cdot \mathbf{T} \cdot\left(\mathbf{F}^{-1}\right)^{T}
$$

where $J$ is the determinant of the deformation gradient, known as the Jacobian [82]. Note the use of the inverse deformation gradient to relate the current configuration 
frame back to the reference frame as we explored in the definition of the deformation gradient, Section 2.1.1.

\section{Equations of Motion}

The behavior and characteristics of the stress tensor are restricted by local conservation equilibrium equations: these two equations are know as the equations of motion.

The first equation of motion is the conservation of linear momentum: the time rate change of the total momentum for a given set of material points equals the sum of the internal tractions and body forces acting on that point set [111]. The equilibrium mathematical expression is

$$
\int_{S} t_{i} d S+\int_{V} \rho b_{i} d V=\frac{d}{d t} \int_{V} \rho v_{i} d V
$$

where we have applied the continuity equation

$$
\frac{d \rho}{d t}=\rho \frac{d v_{i}}{d x_{i}}
$$

to the right hand side of Eq (2.51). Using the definition of the Cauchy stress, Eq (2.49), we can rewrite the linear moment balance as

$$
\int_{S} T_{i j} n_{j} d S+\int_{V} \rho b_{i} d V=\frac{d}{d t} \int_{V} \rho v_{i} d V
$$

After applying the divergence theorem to transform the first term in Eq (2.53) to the volume integral, we obtain Cauchy's first law of motion [26, 111] for an arbitrary 
volume

$$
\frac{\partial T_{j i}}{\partial x_{j}}+\rho b_{j}=\rho \frac{d v_{i}}{d t}
$$

This equation is the governing equation for the quasi-static finite element method simulations we will perform here is a form of Newton's third law of motion [22]. In the absence of body forces and with the assumption of static equilibrium, Eq (2.54), reduces to the well-known stress divergence equation:

$$
\nabla \cdot \mathbf{T}=0
$$

The second equation of motion we will discuss here is the balance of angular momentum. Similar in form to the balance of linear momentum, the angular moment balance states that the sum of the moments of the forces acting on a set of material points must equal the total moment of momentum [111]. Adding a moment arm, $\mathbf{r}$, to each term of the linear momentum balance, Eq (2.51), results in the angular momentum balance expression

$$
\int_{S}(\mathbf{r} \times \mathbf{t}) d S+\int_{V} \rho(\mathbf{r} \times \mathbf{b}) d V=\frac{d}{d t} \int_{V} \rho(\mathbf{r} \times \mathbf{v}) d V
$$

With the deformation of the cross product, after applying the Cauchy stress definition and the divergence theorem as before [22], we can write the balance as

$$
\int_{V} e_{r m n}\left[\frac{\partial\left(x_{m} T_{j m}\right.}{\partial x_{j}}+x_{m} \rho b_{n}\right] d V=\int_{v} e_{r m n} \frac{d}{d t}\left(x_{m} v_{n}\right) d V
$$

Applying the chain rule to the derivative terms, canceling the terms, and applying 
the balance of linear momentum, we arrive at the conclusion

$$
T_{m n}=T n m
$$

for an arbitrary volume [111]. Thus, the stress tensor is required to be symmetric to satisfy the angular moment balance $[82,22,156]$.

We note that although these equations of motion have been demonstrated for the Cauchy stress, they also hold for the other stress measures, including the second Piola-Kirchhoff stress.

These sections have given an overview of the fundamental solid mechanics principles. While the deformation, strain, and stress principles for solid mechanics are established, the development of constitutive equations is a current and exciting area of research. With an understanding of these principles in hand, we will briefly overview the physical structure of metallic crystals before discussing in detail the constitutive laws developed in this work for crystal plasticity.

\subsection{Crystalline Structure of Metals}

This thesis work advances the field by introducing and applying a physically based model for the deformation of two metal materials used within nuclear power plant reactor pressure vessels: the terms in the crystal plasticity constitutive equations are rooted in the physical mechanisms of the metal microstructure evolution. In order to 
discuss these constitutive equations, in Section 2.3, we must first establish a basis of the physical structure of crystalline materials.

We begin our discussion of the microstructure of metals with an overview of the ideal perfect crystal structure. Since both of the metals we study in this work, $\alpha$-iron and nickel-based alloys, have a general cubic crystal structure, we can simplify our discussion of the ideal atomic arrangement within the unit crystal structure cube. We will then broaden our microstructure overview to include common crystal defects, including dislocations which are know as carriers of plasticity. After a discussion of the evolution and interactions of crystal defects within a metal grain, we will conclude this section with a concise review of the impact of lattice curvature on the microstructure, particularly in regards to the grain boundaries. We conclude this section with a discussion of the evolution of dislocations in the microstructure, including interaction of the dislocations with other crystal defects.

\subsubsection{Ideal Unit Crystal Structure}

Crystal structure is defined as the arrangement of atoms in a repeatable, periodic pattern [69]. The structure of a metal crystal governs how the microstructure will evolve as the metal deforms [82]. The metals of interest in this work are both of the cubic type, with each side of the cube having a length $a$. The cubic crystal has an 
atom at each corner of the unit cell. The two metals we will discuss in this work, $\alpha$-iron and nickel, differ in the number and location of the additional atoms within the cubic unit cell. The crystal structure for $\alpha$-iron includes a single additional atom, centered in the body of the unit cell. This structure type is termed Body Centered Cubic (BCC) [64]. Nickel, on the other hand, has a Face Centered Cubic (FCC) structure with an additional atom in the center of each face of the unit cell cube [69]. A collection of unit cubes is termed the crystal lattice [64], and here the term crystal lattice is often used to refer to the larger crystalline structure within a metal.

The concept of Miller indices is used to describe the planes and directions withing a cubic crystal unit cell. These descriptions are necessary to characterize the motion of dislocations, which move along slip planes in slip directions. We will discuss dislocations and their movement in Section 2.2.3.

The Miller indices for a plane are given as the reciprocals of the intersection of the plane with the unit cube reduced to the smallest integer in the ratio [69]. A plane which intersects the unit cell cube at $\left(\frac{1}{2}, \frac{1}{2}, 1\right)$ has the Miller indices of (112). A direction is described in Miller indices by a vector which is parallel to the direction and intersects the origin of the unit cube. The Miller indices of the direction are the three smallest integers in the ratio of the components of the vector resolved on the lengths of the unit cell [69]. A direction parallel to the vector which exits the unit cube at $(1,1,1)$ has the Miller indices of [111]. A negative Miller index is indicated 
by a line drawn over the top of the number. We note that the Miller indices of a plane correspond to the vector normal to the plane [69]. By convention the Miller indices for planes are designated with parentheses, (), while directions are denoted with square brackets, [] [69].

Because of the symmetric nature of the unit FCC and BCC cubes, multiple planes and multiple directions have the same crystallographic type and are grouped into 'families' [64]. The planes $(11 \overline{1}),(1 \overline{1} \overline{1}),(\overline{1} 11)$, and (111) all belong to the family $\{111\}$. Again by convention, the families of planes are denoted with curly braces, \{\} and families of directions are marked with angle brackets $<>$ [69]. The concept of families simplifies the direction of dislocation slip systems by acknowledging the innate symmetry of the cubic crystal structure.

This concept of Miller indices for planes and directions affords us the ability to characterize the two cubic structures of interest in this work. In the BCC unit cell, the $<111>$ direction is the closest packed direction, while in the FCC unit cube the closest packed direction is $<110>$ [69]. The closest packed directions within the two unit cells correspond to the direction of dislocation slip [42] and are examples of how the structure of the crystal dictates the deformation behavior of metals. In the next section we will briefly discuss some of the common defects that may be present in $\alpha$-iron and nickel alloys. 


\subsubsection{Crystal Lattice Defects}

Defects in the crystal lattice are caused by displaced or missing atoms from the lattice sites defined by the unit cell. Hull and Bacon introduce four types of defects: point, line, plane, and volume defects [69]. We will discuss common defects in $\alpha$-iron and nickel from each of these defect types and will introduce the basics of the defect interactions. We will expand on defect interactions and evolution within Section 2.2.3 before introducing mathematical models for these physical mechanisms in Section 2.3.

\section{Point Defects}

Point defects are created when a single atom is displaced from the lattice site in a perfect crystal. The two types of point defects are vacancies and interstitials: vacancy is the name of the empty lattice site from whence the displaced atom originated and interstitial is the name given to the displaced atom itself [69]. These point defects occur in pairs, termed Frankel pairs, although vacancies and interstitials have different rates of absorption into larger defects [194]. Vacancies can also occur in vacancy pairs of opposite sign [69]. The production of point defects in Frenkel pairs is often associated with exposure to radiation, as in a nuclear reactor pressure vessel when nuclear fission products knock atoms out of the lattice sites [194]. 


\section{Dislocations as Line Defects}

Dislocations, which we have already mentioned briefly, are line defects. Dislocations are ideally characterized as either edge or screw dislocations: edge dislocations can be described as an extra half plane inserted into the crystal lattice while screw dislocations have a single surface helicoid shape [69]. In both cases, the dislocation is a line defect in the crystal lattice that defines the boundary between the slipped and unslipped regions of the crystal [64]. The term 'slipped' is used to indicate that portion of the crystal lattice has been deformed by the passage of a dislocation.

Dislocations are characterized by a Burgers vector. The Burgers vector, $b$, is the length required to complete the Burgers circuit, an atom-to-atom closed circuit path in a perfect crystal [64]. Around a single dislocation, the closure failure of the Burgers circuit from a perfect crystal lattice is the Burgers vector [69]. A Burgers vector is defined from a clockwise Burgers circuit, and the Burgers vector of a perfect dislocation corresponds to the shortest lattice transition vector between two atoms [69]. Thus the Burgers vector for a perfect dislocation lies in the closest-packed direction of the unit crystal cell. In a BCC material the Burgers vector is therefore $b=\frac{1}{2}[111]$ while in a FCC material the Burgers vector is $b=\frac{1}{2}[110]$ [65]. The $\frac{1}{2}$ factor in each of the Burgers vector definitions is a result of the energy distribution required to shift a plane of atoms over one lattice site: the maximum energy required occurs at the halfway point between the two lattice points or at half of the lattice parameter, 
$a$, length [69].

The Burgers vector of an ideal edge dislocation is normal to the line of the dislocation itself, such that the edge dislocation moves in the same direction as the Burgers vector [64]. The ideal screw dislocation, on the other hand, has a Burgers vector parallel to the line of the dislocation [65] and normal to the direction of motion of the screw dislocation.

Dislocations cannot end within a crystal and instead either terminate in other dislocations and grain boundaries or form loops within the crystal [69]. As such, the concepts of edge and screw dislocations are idealizations, and most dislocations exist with a mixed edge and screw character. Separating the two types of dislocations allows for a better study of individual behaviors in the idealized state.

Regardless of the dislocation type, the dislocation line and the Burgers vector are contained within the same plane: we term this plane the glide plane [65]. This glide plane is denoted with the Miller indices for the plane normal. Together with the direction vector, traditionally represented also in Miller indices, the glide, or slip, planes describe where in a crystal lattice dislocations can move. We will return to this concept of plane and direction pairs when we discuss dislocation motion in Section 2.2.3. 


\section{Planar Defects}

Planar defects within a crystal are a disruption in the stacking order of atoms within the crystal lattice [69], when we consider the crystal lattice as a series of unit cell cubes stacked on top of each other. The layers of the close packed planes are stacked in a repeating order, e.g. $A B C A B C A$ in $\mathrm{FCC}$ materials. A disruption in this repeating order, e.g. $A B C A B C B A C B A$, is termed a stacking fault [65]. The tendency of the metal to resist stacking faults is known as the stacking fault energy [69].

Metals with low stacking fault energies, like copper and FCC alloys, tend to have a larger presence of partial dislocations which have a spread dislocation core [65, 69]. Conversely materials with a high stacking fault have a more compact dislocation core. A high concentration of partial dislocations can lead to twinning behavior $[64,76,110]$. While we will discuss twinning later in Section 2.2.3, the occurrence of twinning is marked by the presence of a twin boundary, another type of planar defect. A twin boundary is defined by a plane about which the parent and twinned crystal lattices mirror each other [69].

The final type of planar defect we will discuss here is the boundary between two crystalline grains. Up to now, our discussion has remained within a single lattice orientation; however, engineering scale metal structural components are composed of many grains. Each grain has a lattice orientation different from its neighbors, and the grain boundary plane is characterized by a misorientation angle [69]. 
Although the grain boundary region itself is narrow, the impact of the grain boundary on the behavior of dislocations can be significant. Because of the change in lattice orientations, dislocations cannot directly traverse the grain boundary in the same manner as they travel across the crystal within the grain. Furthermore, even approaching the grain boundary is energetically difficult for dislocations because the stress fields near the grain boundary are high [65]. Dislocations within the crystal lattice form pile-up structures, akin to a traffic jams, in response to the barrier of the grain boundary, increasing the resistance strength to dislocation motion of the crystal lattice [82]. The degree of resistance to dislocation motion provided by the grain boundary depends on the degree of misorientation, or the curvature of the lattice across the grain boundary. In general metallic materials with smaller grains have higher strength than metals with larger grains; this relationship is captured on the engineering scale by the Hall-Petch relation $[148,62]$. We will dicuss the effect of grain boundaries in the context of modelling lattice curvature in Section 2.3.5.

\section{Volumetric Defects}

The final class of crystalline defects we will discuss here is the volume defect type. As the name suggests, these defects have a 3D form and are often the result of point defects coalescing. There are many different types of volumetric defects, yet in this work will will mention only four: self-interstitial atom (SIA) dislocation loops, stacking fault tetrahedrons (SFTs), voids, and ordered precipitates. We will 
also discuss these defects only withing the context of irradiation damage even though these defects can occur under other circumstances.

When a fission product knocks an atom in the metal crystal lattice out of place, it causes a displacement cascade which produces a group of vacancies surround by a shell of interstitial atoms [194]. Either group may collapse into a region of a stacking fault [69]. In-cascade clusters of point defects may also occur during the cascade event [194], and the structures formed by the clusters depend heavily on the crystal structure $[143,173]$.

In a BCC material such as $\alpha$-iron self interstitials clusters into crowdions which form SIA loops with Burgers vectors along the $<111>$ or $<100>$ directions [194, 17]. SIA loops, which are considered to be the most significant irradiation defect in $\alpha$-iron $[54,167]$ interact as a weak barrier to mobile dislocations. Since these SIA loops have a 3D character, these defects affect the movement of dislocations within a sphere of influence [114]. Interactions with mobile dislocations cause the SIA loops to unfault $[17,194]$. As a dislocation loop, the majority of SIA loops are mobile, yet the larger SIA loops containing more than a few interstitial atoms rely on thermal diffusion to glide in one dimension and are therefore relatively slow [194]. Clusters of vacancies can also form loops in $\alpha$-iron, but these vacancy loops are unstable and unfault after a critical number of vacancies join the loop [143].

In FCC materials, particularly those with low stacking fault energies such as 
nickel alloys, clusters of vacancies and Frank loops will form three partial Shockely dislocations. These partial dislocations travel on the three $\{111\}$ type planes to intersect and thereby form a 3D SFT [194, 69]. SFTs are important irradiation defects in FCC materials, comprising 25 to 50 percent of all the irradiation clusters in nickel [14]. SFTs are immobile defects, and dislocations interact with these defects by cutting through the SFT defect [194].

Voids occur in both BCC and FCC materials and are formed by the coalescence of vacancies [194]. Generally voids are considered strong barriers to dislocation motion, and a dislocation must either cut through or bow around a void [139]. Precipitates are similar to voids in that they are 3D clusters but are composed of either solute atoms or different phases of the metal or alloy of interest. The higher levels of lattice disturbance characteristic of a radiation environment allows for the formation of these phases or solute atom collections [194]. Within nickel-based alloys, long-range ordered (LRO) $\gamma^{\prime}$ precipitates form under aging and radiation conditions. Similar to voids, precipitates act as strong barrier obstacles for dislocation motion [194], and either are sheared by dislocations or act to pin dislocations and prevent dislocation movement. The process of cutting through a precipitate is made more difficult by the presence of any lattice incoherency between the matrix crystal lattice and the lattice of the precipitate.

As we've alluded to in this section, the impact of these different types of crystal 
lattice defects is most strongly felt when the dislocations move to enable plastic deformation of the metal.

\subsubsection{Dislocations and Plasticity}

Metals deform plastically in an incompressible manner; plastic deformation does not create a volume change [82]. Furthermore plastic deformation occurs only in response to shear loading and not to hydrostatic loading [42]. This sensitivity to only shear loading is a direct result of how dislocations move within the crystal lattice of a metal. Hence dislocations are often termed carriers of plasticity. Dislocations are the lattice defect that $[179,151,141]$ all independently established is needed to explain why typical metal crystals plastically slip at much lower stresses than are theoretically predicted and measured for an ideal perfect crystal [64].

Mathematically, this relationship between plastic strain and dislocation motion was formalized in the Orowan relation. The Orowan relation establishes the rate of plastic slip, $\dot{\gamma}^{P}$, in recognition of the shear-only plastic deformation of the metals, as a function of the total rate of dislocation motion [141]:

$$
\dot{\gamma}^{P}=\rho_{\text {mobile }} b v
$$

where $\rho_{\text {mobile }}$ is the density of mobile dislocations, $b$ is the Burgers vector, and $v$ is the velocity of the mobile dislocations. This relationship, however, is written only for 
a set of dislocations moving in the same slip direction on the same glide plane. Recall in Section 2.2.2 that we introduced the concept of slip planes and slip directions.

Dislocations move with two mechanisms: conservative glide and nonconservative climb. Dislocation glide occurs in the the glide, or slip plane, and for edge dislocations, in the slip direction. Therefore, the combination of a slip plane, $(\hat{s})$, and a slip direction, $(\hat{m})$ is termed a slip system [65]. In a BCC material, microscopic evidence suggests that the slip plane famlies are $\{110\}$ and $\{112\}[69]$ and the closed packed slip directions are $<111>$. While only the $\{110\}$ slip planes are active at very low temperatures, at midrange temperatures BCC materials like $\alpha$-iron have 24 potential active slip systems, as shown in Table 2.1. At higher temperatures, an additional 24 $\{123\}$ slip planes become active [145]. In an FCC material, only the $\{111\}$ planes are slip planes, and the closed packed slip direction, $<110>$, yields a total of 12 slip systems, Table 2.2 .

The velocity of the dislocation becomes nonzero when a sufficiently large shear force is placed on the dislocations. In 1959 Johnston and Gilman established the phemonological relationship between dislocation velocity and the applied shear stress through a series of careful experimental measurements[73]:

$$
v \propto\left(\frac{\tau}{\tau_{o}}\right)^{n}
$$

where $\tau$ is the applied shear stress on the slip system in the slip direction, $\tau_{o}$ is the resistance of the crystal lattice to dislocation motion, and $n$ is an exponent dependent 
Table 2.1: Glide slip systems in BCC crystals

\begin{tabular}{|c|c|c|c|}
\hline Slip Plane & Slip Direction & Slip Plane & Slip Direction \\
\hline$(011)$ & {$[1 \overline{1} 1]$} & $(112)$ & {$[11 \overline{1}]$} \\
\hline$(011)$ & {$[11 \overline{1}]$} & $(\overline{1} 12)$ & {$[1 \overline{1} 1]$} \\
\hline (101) & {$[\overline{1} 11]$} & $(1 \overline{1} 2)$ & {$[\overline{1} 11]$} \\
\hline$(101)$ & {$[11 \overline{1}]$} & $(11 \overline{2})$ & {$[111]$} \\
\hline (110) & {$[\overline{1} 11]$} & $(121)$ & {$[1 \overline{1} 1]$} \\
\hline (110) & {$[1 \overline{1} 1]$} & $(\overline{1} 21)$ & {$[11 \overline{1}]$} \\
\hline$(0 \overline{1} 1)$ & {$[111]$} & $(1 \overline{2} 1)$ & [111] \\
\hline$(0 \overline{1} 1)$ & {$[\overline{1} 11]$} & $(12 \overline{1})$ & {$[\overline{1} 11]$} \\
\hline$(10 \overline{1})$ & {$[111]$} & $(211)$ & {$[\overline{1} 11]$} \\
\hline$(10 \overline{1})$ & {$[1 \overline{1} 1]$} & $(\overline{2} 11)$ & {$[111]$} \\
\hline$(\overline{1} 10)$ & {$[111]$} & $(2 \overline{1} 1)$ & {$[11 \overline{1}]$} \\
\hline$(\overline{1} 10)$ & {$[11 \overline{1}]$} & $(21 \overline{1})$ & {$[1 \overline{1} 1]$} \\
\hline
\end{tabular}

on the material. The applied shear stress, $\tau$, causes dislocation motion when it reaches the value of the critical resolved shear stress $[69,11]$. The applied shear stress is resolved from a Rank-2 stress tensor, like the Cauchy or second Piola-Kirchhoff stress tensors from Section 2.1.4, by the Schmid factor to the slip plane in the slip direction:

$$
\tau=\mathbf{T}: \mathbf{M}=\mathbf{T}: \hat{\mathbf{s}} \otimes \hat{\mathbf{m}}
$$


Table 2.2: Glide slip systems in FCC crystals

\begin{tabular}{cccc} 
Slip Plane & Slip Direction & Slip Plane & Slip Direction \\
$(111)$ & {$[\overline{1} 01]$} & $(1 \overline{1} 1)$ & {$[011]$} \\
$(111)$ & {$[\overline{1} 10]$} & $(1 \overline{1} 1)$ & {$[110]$} \\
$(111)$ & {$[0 \overline{1} 1]$} & $(1 \overline{1} 1)$ & {$[\overline{1} 01]$} \\
$(\overline{1} 11)$ & {$[101]$} & $(\overline{1} \overline{1} 1)$ & {$[011]$} \\
$(\overline{1} 11)$ & {$[110]$} & $(\overline{1} \overline{1} 1)$ & {$[\overline{1} 10]$} \\
$(\overline{1} 11)$ & {$[0 \overline{1} 1]$} & $(\overline{1} \overline{1} 1)$ & {$[101]$} \\
\hline
\end{tabular}

The denominator of Eq 2.60, $\tau_{o}$ is the Peierls-Nabarro stress. Also referred to as the lattice friction, the Peierls-Nabarro stress is the amount of applied shear stress required to move the atoms in the dislocation half a lattice parameter, or half the distance between two atom lattice site positions [69].

Cross slip is a significant component of dislocation motion in BCC materials [152]. In cross slip, screw dislocations move from one slip plane to another with the same slip direction [69]. Slip, or glide, planes with the same direction are termed cross slip families; these families are given in Table 2.3 for BCC materials for medium temperatures in which only the $\{110\}$ and $\{112\}$ slip planes are active. At higher temperatures, when the $\{123\}$ slip planes becomes active in BCC materials, and these planes also contribute to the cross slip families. The contribution to cross slip families 
from the $\{123\}$ planes are not listed in Table 2.3. FCC materials only have two slip planes per slip direction.

Table 2.3: Cross slip families in BCC crystals

\begin{tabular}{|c|c|c|c|}
\hline Slip Plane & Slip Direction & Slip Plane & Slip Direction \\
\hline$(011)$ & {$[1 \overline{1} 1]$} & $(101)$ & {$[\overline{1} 11]$} \\
\hline$(110)$ & {$[1 \overline{1} 1]$} & $(110)$ & {$[\overline{1} 11]$} \\
\hline$(10 \overline{1})$ & {$[1 \overline{1} 1]$} & $(0 \overline{1} 1)$ & {$[\overline{1} 11]$} \\
\hline$(\overline{1} 12)$ & {$[1 \overline{1} 1]$} & $(1 \overline{1} 2)$ & {$[\overline{1} 11]$} \\
\hline$(121)$ & {$[1 \overline{1} 1]$} & $(12 \overline{1})$ & {$[\overline{1} 11]$} \\
\hline$(21 \overline{1})$ & {$[1 \overline{1} 1]$} & $(211)$ & {$[\overline{1} 11]$} \\
\hline Slip Plane & Slip Direction & Slip Plane & Slip Direction \\
\hline$(011)$ & {$[11 \overline{1}]$} & $(0 \overline{1} 1)$ & {$[111]$} \\
\hline (101) & {$[11 \overline{1}]$} & $(10 \overline{1})$ & {$[111]$} \\
\hline$(\overline{1} 10)$ & {$[11 \overline{1}]$} & $(\overline{1} 10)$ & {$[111]$} \\
\hline$(112)$ & {$[11 \overline{1}]$} & $(11 \overline{2})$ & {$[111]$} \\
\hline$(\overline{1} 21)$ & {$[11 \overline{1}]$} & $(1 \overline{2} 1)$ & {$[111]$} \\
\hline$(2 \overline{1} 1)$ & {$[11 \overline{1}]$} & $(\overline{2} 11)$ & {$[111]$} \\
\hline
\end{tabular}


In addition to dislocation glide, dislocations may also move by climb. Climb is known as a nonconservative motion because it relies on the thermal diffusion of vacancies to enable the dislocation to travel out of the glide plane in a direction perpendicular to the glide plane [69].

\section{Defect Hardening of the Crystal}

Defects in the metal crystal microstructure impede the motion of dislocations by disrupting the crystal lattice through which the dislocations travel. Defects can also pin a dislocation to prevent it from moving; we term these dislocations which cannot move past an obstacle immobile dislocations. Sometimes, under additional applied shear stress, these dislocations can become mobile again. We will discuss the coupling between mobile and immobile dislocations in greater detail in Section 2.3.2.

Generally, defects in the crystal impede but do not prevent the motion of dislocations. Other dislocations on different slip systems can act as an obstacle to a mobile dislocation; this hardening due to the presence of other dislocations is termed forest hardening [69]. As a result of the presence of these defects, an increased applied shear stress must be placed on the dislocation to overcome these defect obstacles. Increased numbers of microstructure defects naturally leads to higher shear stress requirement to produce dislocation motion; the increased resistance of the crystal to dislocation motion is termed hardening or strengthening [64]. Having introduced the different types of dislocation defects interactions in this section, we will continue the discussion 
of the mathematical models for these interactions in Section 2.3.4.

\section{Dislocation Generation}

The evolution of the microstructure involves generation of new dislocations in addition to the interaction of dislocations with other defects as we have previously discussed. A common dislocation generation mechanism is the Frank-Read source [69]. In a Frank-Read source, a dislocation segment is pinned by other defects and bows out under applied shear stress. The pinned segment continues to bow out until the dislocation segment comes into contact with itself on the opposite side of the pins [64], as shown in Figure 2.3. At this point the two curved components of

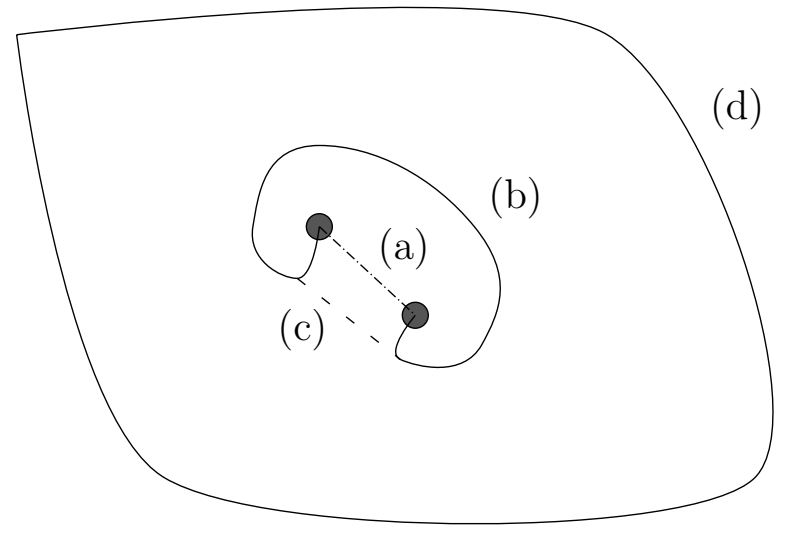

Figure 2.3: The Frank-Read dislocation generation source, line (a), operates under an applied shear stress which causes the pinned dislocation to bow out, line (b), until the line at (c) is minimized. At that point, a new dislocation loop, line (d), is released and the dislocation source, line (a) is restored. 
the dislocation segment have opposite character from each other. When these two segments come into contact, they annihilate and release a perfect dislocation loop and a dislocation segment pinned as before [69]. Continued applied shear stress will grow the new dislocation loop and will enable the Frank-Read dislocation segment source to generate yet another dislocation loop.

\subsection{Crystal Plasticity Framework}

The mathematical path connecting the motion and interactions of metallic crystal dislocations and defects is not straightforward. Three different researchers, marking the start of research into how the microstructure of a metal crystal impacts the engineering scale behavior, independently verified that the theoretical slip system strength significantly overpredicts the observed strength of a crystalline metal [141, 151, 179]. Since then the field of crystal plasticity has developed from averaged single crystal responses [180] to phenomenological expressions for dislocation evolution under stress [85] and relations for lattice curvature [128] to complex nonlinear approaches that include assorted dislocation movement mechanisms which account for interactions with other crystal defects $[116,161]$. 


\subsubsection{Constitutive Equations for Plasticity}

Following [11] our crystal plasticity model uses a multiplicative decomposition of the deformation gradient into elastic and plastic components:

$$
\mathbf{F}=\mathbf{F}^{e} \mathbf{F}^{p}
$$

The change in the crystal shape due to dislocation motion is accounted for in the plastic deformation gradient tensor, $\mathbf{F}^{p}$, while the elastic deformation gradient tensor, $\mathbf{F}^{e}$, accounts for recoverable elastic stretch and rotations of the crystal lattice. The evolution of the plastic deformation is given as

$$
\dot{\mathbf{F}}^{p}=\mathbf{L}^{p} \mathbf{F}^{p}
$$

where $\mathbf{L}^{p}$ is the plastic velocity gradient. The plastic deformation gradient rate is used to calculate the increment of the Lagrangian strain. The plastic velocity gradient is defined as the sum of the slip increments from dislocation motion on all of the slip systems [11].

$$
\mathbf{L}^{\mathbf{p}}=\sum_{\alpha=1}^{n} \dot{\gamma}^{\alpha} \mathbf{s}_{o}^{\alpha} \otimes \mathbf{m}_{o}^{\alpha}
$$

where $\dot{\gamma}^{\alpha}$ is the slip rate due to dislocation glide. Note that the slip direction and slip plane normal unit vectors, $\mathbf{s}_{o}$ and $\mathbf{m}_{o}$, are defined in the reference configuration. This relationship links the continuum framework to the constitutive models used in crystal

plasticity. The plastic dislocation glide slip on each slip system, $\dot{\gamma}^{(\alpha)}$, is connected to 
the behavior of the mobile dislocations through Orowan's relation, Eq 2.59.

The evolution of the mobile dislocation rate is a constitutive expression, described in 2.3.2 below. At the continuum crystal plasticity level, the dislocation glide velocity is computed as a function of the applied shear stress on the slip system $\alpha$ and each slip system strength. In this work, we apply a power law expression for the dislocation glide velocity,

$$
v_{\text {glide }}^{\alpha}=v_{o}\left|\frac{\tau^{\alpha}}{g^{\alpha}}\right|^{1 / m} \operatorname{sign}\left(\tau^{\alpha}\right) \text { if }\left|\tau^{\alpha}\right| \geq g_{o}^{\alpha}
$$

where $v_{o}$ is the initial dislocation velocity, $\tau^{\alpha}$ is the applied resolved shear stress on each slip system $\alpha$, and $g^{\alpha}$ is the slip system resistance or strength which is further defined in Eq (2.70). The power law expression for dislocation glide velocity, while simple and neglecting direct temperature dependence, offers significant robustness over an enthaply flow type expression [28, 145]; the enthalpy flow velocity glide is numerically sensitive to the parameter choices and requires additional numerical stablization schemes [29]. As a consequence of the decision to use the slip direction and plane normal unit vectors from the initial crystal orientation, Eq (2.64), the second Piola-Kirchoff stress is used to determine the applied resolved shear stress.

$$
\tau^{\alpha}=\mathbf{T}: \mathbf{s}_{o}^{\alpha} \otimes \mathbf{m}_{o}^{\alpha}
$$

The slip system resistance, $g^{\alpha}$ in Eq (2.65), provides a measure of the strength of each slip system to resist dislocation motion. 


\subsubsection{Dislocation Evolution Equations}

\subsubsection{Dislocation Evolution}

Our crystal plasticity model is based on the continuum dislocation dynamics (CDD) framework, [101, 177], with separate terms used to describe each of the specific physical interaction mechanisms in the dislocation evolution rate terms. The rate of the mobile dislocation evolution is fully coupled to the immobile dislocation evolution rate. The mobile dislocation evolution is governed by six terms: each term in the equation represents a specific physical dislocation-interaction mechanism.

$$
\begin{aligned}
\dot{\rho}_{\text {mobile }}^{(\alpha)}= & \dot{\rho}_{\text {generation }}^{(\alpha)}-\dot{\rho}_{\text {mobile-annihilation }}^{(\alpha)}-\dot{\rho}_{\text {locking }}^{(\alpha)}+\dot{\rho}_{\text {freed }}^{(\alpha)} \\
& +\dot{\rho}_{\text {cross-slip }}^{(\alpha)}-\dot{\rho}_{\text {immobile-annihilation }}^{(\alpha)}
\end{aligned}
$$

The mathematical relations for each of the terms in the general mobile dislocation evolution expressions are given in Table 2.4.

Mobile dislocation generation is considered to occur from Frank-Read sources. Dislocation annihilation of two dislocations of opposite sign can occur between two mobile dislocations (second term in Eq. (2.67)) or between an immobile dislocation and a mobile dislocation (sixth term in Eq. (2.67)). The annihilation interaction among dislocations is assumed to occur only within a capture radius, $R_{c}$, which is defined as a factor of the Burgers vector. The mobile dislocation evolution is coupled to the immobile dislocation evolution rate through evolution terms for dislocation locks 
Table 2.4: Mathematical expressions for the six physically-based interaction mechanisms used within the mobile dislocation evolution equation, Eq (2.67), and immobile dislocation evolution equation, Eq (2.68). The six $\alpha$ coefficients are fit from dislocation dynamics simulations [101], $v_{\text {glide }}$ is the dislocation glide velocity from $\mathrm{Eq}(2.65), R_{c}$ is the radius of capture for annihilating dislocations of opposite sign, and $l_{i n v}$ is the inverse mean free glide path.

\begin{tabular}{lcl}
\hline Dislocation Interaction & Mathematical Expression \\
\hline Frank-Read generation & $\dot{\rho}_{\text {generation }}^{(\alpha)}=\alpha_{1} \rho_{\text {mobile }}^{(\alpha)} v_{\text {glide }} l_{\text {inv }}$ \\
Mobile-mobile annihilation & $\dot{\rho}_{\text {mobile-anhl }}^{(\alpha)}=\alpha_{2}\left(\rho_{\text {mobile }}^{(\alpha)}\right)^{2} 2 R_{c} b v_{\text {glide }}$ \\
Dislocation locking & $\dot{\rho}_{\text {locking }}^{(\alpha)}$ & $=\alpha_{3} \rho_{\text {mobile }}^{(\alpha)} v_{\text {glide }} l_{\text {inv }}$ \\
Locked dislocations freed & $\dot{\rho}_{\text {freed }}^{(\alpha)}$ & $=\alpha_{4} \rho_{\text {imm }}^{(\alpha)}\left|\frac{\tau^{(\alpha)}}{g^{(\alpha)}}\right|^{2} v_{\text {glide }} l_{\text {inv }}$ \\
Cross slip & $\dot{\rho}_{\text {cross-slip }}^{(\alpha)}$ & $=\alpha_{5} \rho_{\text {cross-slipped }}^{(\alpha)} v_{\text {glide }} l_{\text {inv }}$ \\
Immobile-mobile annihilation & $\dot{\rho}_{\text {imm-anhl }}^{(\alpha)}$ & $=\alpha_{6} \rho_{\text {mobile }}^{(\alpha)} \rho_{\text {imm }}^{(\alpha)} 2 R_{c} b v_{\text {glide }}$ \\
\hline
\end{tabular}

and dislocations freed from locks (third and fourth terms in Eq (2.67), respectively). These terms also appear in the evolution rate equation for immobile dislocations

$$
\dot{\rho}_{\text {immobile }}^{(\alpha)}=\dot{\rho}_{\text {locking }}^{(\alpha)}-\dot{\rho}_{\text {freed }}^{(\alpha)}-\dot{\rho}_{\text {immobile-annihilation }}^{(\alpha)}
$$

The locked dislocations term, which is negative in the mobile dislocation evolution 
equation, Eq (2.67), acts as the source term for the immobile dislocations. The cross slip term in the mobile dislocation evolution, $\mathrm{Eq}$ (2.67), allows mobile dislocations to move from one slip system to another slip system within the same cross slip family, depending on the applied shear stress.

The inverse mean glide path, $l_{\text {inv }}$ in Table 2.4 , is a measure of the space among existing dislocations through which a mobile dislocation could glide [135]. The inverse mean glide path represents the increased difficultly of mobile dislocation glide as the total dislocations accumulate within the grain; with increased numbers of accumulated dislocations the space between accumulated dislocations decreases.

$$
l_{\text {inv }}^{(\alpha)}=\beta_{\text {path }} \sqrt{\sum_{\beta} \omega^{(\alpha \beta)}\left(\rho_{\text {mobile }}^{(\beta)}+\rho_{\text {immobile }}^{(\beta)}\right)}
$$

where $\beta_{\text {path }}$ is a fitting coefficient. This mean free glide path quantity is used as an inhibiting agent in the dislocation evolution terms listed in Table 2.4.

\subsubsection{Interaction of Dislocations and Other Defects}

Physically-based frameworks write the constitutive slip system resistance equation as a function of dislocation and defect densities within the crystal [135, 161]; both defects and other dislocations act as barriers to dislocation motion [65, 204]. In this model the resistance of the slip systems to dislocation motion is considered as the additive sum of the physical barriers to dislocation motion, including dislocation 
forests, and irradiation defects:

$$
g^{(\alpha)}=g_{o}^{(\alpha)}+g_{\text {forest }}^{(\alpha)}+g_{\text {irradiation-defects }}^{(\alpha)}
$$

The slip system resistance due to dislocation forest-type accumulation on all slip systems is modeled with a modified Bailey-Hirsch approach [15], following [135]:

$$
g_{\text {forest }}^{(\alpha)}=\alpha_{m b h} b \mu \sqrt{\sum_{\beta} \Omega^{(\alpha \beta)}\left(\rho_{\text {mobile }}^{(\beta)}+\rho_{\text {immobile }}^{(\beta)}\right)}
$$

where the hardening is termed self-hardening when $\alpha=\beta$ and latent-hardening when $\alpha \neq \beta$. The coefficient $\alpha_{m b h}$ is a fitting parameter, $b$ is the Burgers vector, $\mu$ is the shear modulus of the material, and $\Omega$ is the interaction matrix containing the matrix of self- and latent-hardening parameters.

\section{Irradiation Defect Evolution in BCC Metals}

The irradiation defects contribute to the embrittlement of the slip systems by

raising the slip system resistance, denoted as $g_{\text {irradiation-defects }}^{(\alpha)}$ in Eq (2.70). In $\alpha$-iron RPV steel self-interstitial atom (SIA) loops act as barriers to dislocation motion. The contribution of the SIA loops is modeled by accounting for the 3D nature of the SIA loop interaction as a barrier to dislocations.

$$
g_{\text {irradiation-defects }}^{(\alpha)}=\alpha_{\text {sia }} \mu\left(\frac{b^{2} d_{\text {sia }}}{l_{\text {sia }}^{3}}\right)^{1 / 2} \quad \text { where } l_{\text {sia }}=\frac{1}{\sqrt[3]{\rho_{\text {sia }}}}
$$

The cubed root dependence of the mean free glide path type term in Eq (2.72) indicates the ability of the SIA loops to inhibit the motion of dislocations in a sphere 
around the SIA loop. SIA loops can also be absorbed by dislocations which pass through the same plane on which a SIA loop exists. Following [114], the evolution of SIA loops is considered with a simple interaction model similar to the dislocation annihilation terms in Table 2.4 .

$$
\dot{\rho}_{\text {sia }}=-4 \beta_{\text {sia }} R_{\text {sia }} \rho_{\text {sia }} \sum_{\alpha} \rho_{\text {mobile }}^{(\alpha)} v_{\text {glide }}^{(\alpha)}
$$

where $\beta_{\text {sia }}$ is a fitting parameter for SIA loop annihilation, $R_{\text {sia }}$ is the radius within which a dislocation can capture and absorb a dislocation and $\rho_{\text {sia }}$ is the density of SIA loops. After an initial exposure to irradiation, the density of SIA loops will decrease under mechanical loading of the crystal as dislocation absorb the SIA loops during glide motion.

\section{Thermal Aging Defect Evolution in FCC Metals}

The use of nickel alloys for piping materials in chemical industry processing plants over decades provides a set of information on the in operation aging of commercial nickel alloys. Several studies have analysized the microstructure of nickel-based alloys after 50,000 hrs to $70,000 \mathrm{hrs}$ of in service operation at temperatures of $450^{\circ} \mathrm{C}$ to $600^{\circ} \mathrm{C}[184,175,164,193,123]$. Among the several precipitates and particles which form in these complex alloys is the formation of long ranged ordered (LRO) $\mathrm{Ni}_{2} \mathrm{Cr}$ precipitates [112]. These coherent precipitates form within nickel-based alloys after long term exposure to relatively low temperatures [200]. Alloying components such 
as iron and molybdenum will shift the temperature range and aging time required for these LRO precipitates to form $[122,188]$, yet these precipitates persist as a microstructural feature in nickel alloys. These LRO precipitates are also know to increase the hardness of nickel-based alloys [112, 201].

The extensive aging time to form the LRO precipitates in commercial nickel-based alloys is not conducive to studying the impact of LRO precipitates on microstructures. Many research groups have thus selected model binary alloys, which form the LRO precipitates after reasonable aging times [113, 183, 200, 172]. These studies connect the formation of $\mathrm{LRO} \mathrm{Ni}_{2} \mathrm{Cr}$ precipitates with increased hardness of the model alloy. Generally 10,000 hrs has been determined to be enough aging time to allow for the complete formation of the LRO precipitates in the binary model alloy [183].

The $\mathrm{Ni}_{2} \mathrm{Cr}$ model alloy only demonstrates tertiary LRO precipitates, under $10 \mathrm{~nm}$ in size. Based on this size, we have implemented a model for weakly coupled APB shearing [89]. The effective spacing between two LRO precipitates is modeled in a manner that accounts for the radii of the precipitates.

$$
g_{a p b}^{(\alpha)}=\frac{\gamma_{a p b}}{2 b}\left[\left(\frac{\gamma_{a p b} \cdot d_{L R O}}{\mu}\right)^{1 / 2} \frac{d_{L R O}}{b\left(L_{s}-d_{L R O}\right)}-\frac{\pi}{4}\left(\frac{d_{L R O}}{L_{s}}\right)^{2}\right]
$$

where $\gamma_{a p b}$ is the energy of the anti-phase boundary, $b$ is the Burgers vector, $d_{L R O}$ is the mean planar diameter of the precipitate, $\mu$ is the shear modulus, $L_{s}$ is the effective spacing between precipitates, and $f$ is the volume fraction of the tertiary precipitates. The effective spacing between precipitates takes into account the diameter of the 
precipitates, [89]:

$$
L_{s}=\left(\frac{8}{3 \pi f}\right)^{1 / 2} d_{L R O}
$$

The weakly coupled terminology refers to the ability of the precipitate to contain only a single dislocation partial at a time; larger precipitates which can contain both partials of a dislocation are said to undergo strongly coupled APB shearing.

\subsubsection{Geometrically Necessary Dislocations}

In this work we calculate the density of the geometrically necessary dislocations (GNDs) form the Nye's dislocation density tensor, $\boldsymbol{\alpha}$. As has been discussed previously and in other published literature, the Nye's dislocation tensor is a measure of the curvature in the lattice [128] through the closure failure due to dislocation accumulation $[128,36]$. Kröner extended this theory to a continuum application from the individual glide plane and dislocations which Nye considered [90, 91]. Ashby first coined the term Geometrically Necessary Dislocations (GNDs), which we employ here, for these curvature dislocations [12]. Following [166] we compute the Nye's tensor [128] from the plastic velocity gradient

$$
\alpha \approx \int_{t}^{t+d t} \nabla_{X} \cdot L^{P} d t
$$

where the dislocation tensor $\alpha$ is used to directly compute a measure of GNDs and the plastic velocity gradient tensor, $L^{P}$, is either a local quadrature point value or 
a smoothed value from the element nodes, depending on numerical implementation selected.

\subsubsection{Dislocation Twinning Volume Fraction Evolution}

Given the small size of the precipitates in the binary $\mathrm{Ni}_{2} \mathrm{Cr}$ model alloy, about 10 nm, we study in this work, the microtwinning models often proposed for commercial alloys are not appropriate for the material. As such, we have elected to implement the twin volume fraction model proposed by [76]. Following Kalidindi's approach, the twinning model is given as [76].

$$
\dot{f}_{\text {twin }}^{\beta}=\frac{\dot{\gamma}}{\gamma_{\text {twin }}}\left(\frac{\tau^{\beta}}{g_{\text {twin }}^{\beta}}\right)^{1 / m} \text { if } \tau^{\beta}>0
$$

This model tracks the evolution of the deformation twin volume fraction, $\dot{f}^{\beta}$ instead of the twin slip rate in a form similar to the power law glide dislocation velocity model, Eq (2.65). This additional slip term in included in the calculation of the plastic velocity gradient. Because the mechanisms of twinning are not well understood, the power law model is an appealing approach to capturing the effect of twinning [161]. 
Table 2.5: Twinning systems in FCC crystals

\begin{tabular}{cccc}
\hline Slip Plane & Slip Direction & Slip Plane & Slip Direction \\
$(111)$ & {$[\overline{2} 11]$} & $(1 \overline{1} 1)$ & {$[\overline{2} \overline{1} 1]$} \\
$(111)$ & {$[1 \overline{2} 1]$} & $(1 \overline{1} 1)$ & {$[121]$} \\
$(111)$ & {$[11 \overline{2}]$} & $(1 \overline{1} 1)$ & {$[1 \overline{1} \overline{2}]$} \\
$(\overline{1} 11)$ & {$[211]$} & $(\overline{1} \overline{1} 1)$ & {$[2 \overline{1} 1]$} \\
$(\overline{1} 11)$ & {$[\overline{1} \overline{2} 1]$} & $(\overline{1} \overline{1} 1)$ & {$[\overline{1} 21]$} \\
$(\overline{1} 11)$ & {$[\overline{1} \overline{1}$} & $(\overline{1} \overline{1} 1)$ & {$[\overline{1} \overline{1} 2]$} \\
\hline
\end{tabular}

In an FCC material, the $\{111\}$ planes are also the twinning planes, and the twin direction, $<112>$, yields a total of 12 twin systems, Table 2.5 .

\subsection{Numerical Implementation in MOOSE}

This crystal plasticity model has been implemented in INL's MOOSE software environment. MOOSE is a Multiphysics Object Oriented Software Environment for FEM modeling to solve coupled physics simulations [57]. Our use of the PJFNK preconditioner and solver functionality offered in MOOSE enables us to run simulations with slightly off Jacobians rather than requiring exact Jacobians. This ability frees our implementation from the constraints imposed by required calculations of the 
Jacobian of the governing equations, the difficulty of which is discussed by [104].

We have implemented the CDD crystal plasticity model within the MOOSE tensor mechanics module. The evolution equations are implemented in an updated Lagrianian incremental form, and a trial stress increment is predicted at each simulation iteration on each of the quadrature points. Using a Newton Rhapson approach, outlined in Figure 2.5, we consider the system convergence when the residual of the second Piola-Kirchhoff stress increments from the current and previous iterations is within tolerances specified by the user. The converged Cauchy stress value and the corresponding strain are then calculated, as shown in Figure 2.4, before being passed back to MOOSE for the FEM integration residual calculation. This Cauchy stress residual calculation is based on the stress divergence theorem, Eq (2.55), and MOOSE reduces the residual from the left-hand side of this equation to the specified tolerance. The Newton-Rhapson iteration algorithm implemented in MOOSE separates the iteration over the second Piola-Kirchhoff stress residual from the physically based constitutive models used to calculate the plastic velocity gradient, Figure 2.5. The convergence algorithm for Newton-Rhapson iteration is taken from other approaches already implemented in MOOSE and is adapted for our crystal plasticity framework. 


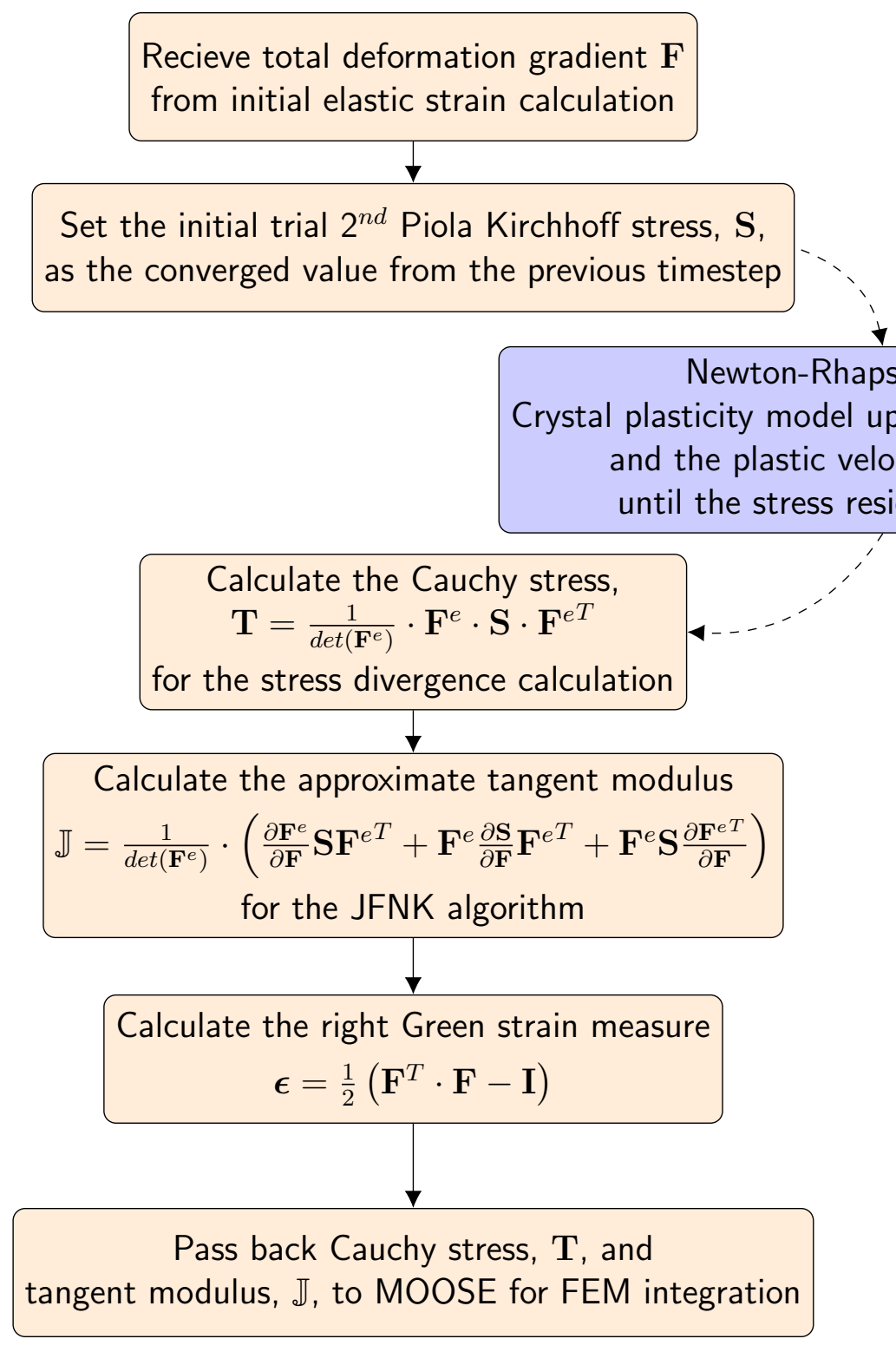

Figure 2.4: The flowchart for the calculation of the stress and strain measures within the CrystalPlasticityUpdate class as implemented in the tensor mechanics module of MOOSE is shown here. The components involved in the Newton-Rhapson iteration are shown in light blue and expanded in Figure 2.5, and the components shown in light orange are executed once per MOOSE iteration. 


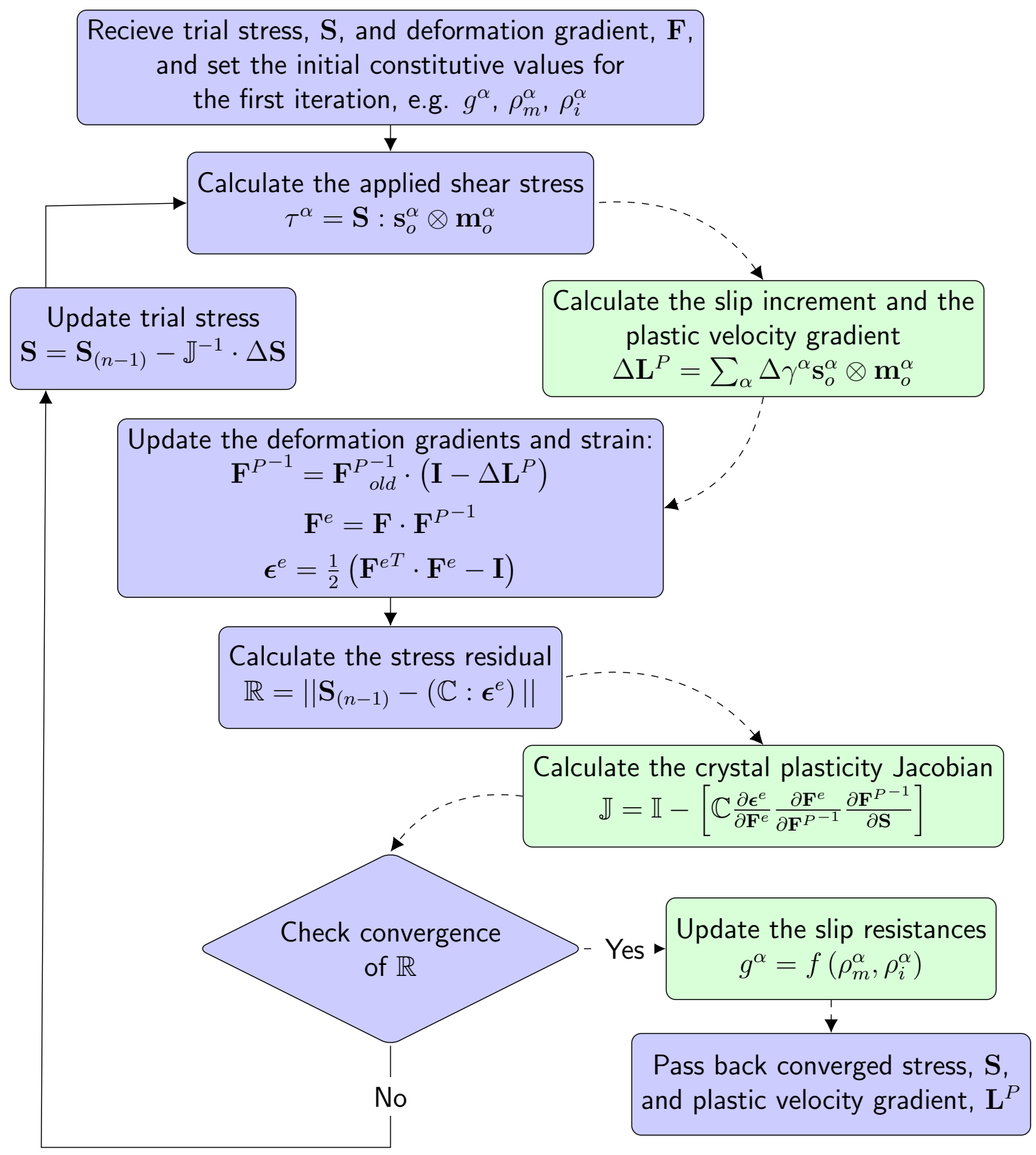

Figure 2.5: The algorithm components of the Newton-Rhapson iteration are shown in light blue and the crystal plasticity constitutive model components are shown in light green. 
The code developed in this work includes both algorithms shown in Figures 2.4 and 2.5. The initial implementation of the algorithm in Figure 2.4 was completed within another MOOSE class [29]. In this work we have migrated this algorithm, and the Newton-Rhapson iteration, shown in Figure 2.5, to a new type of MOOSE code class. This new code class, the Stressupdate classes, is designed to perform an internal iteration. To return a trial stress to the yield surface, the internal iteration ability of the StressUpdate classes is best suited for our use case here.

In this work we have focused on expanding the physically based crystal plasticity constitutive models, indicated by the light green components in Figure 2.5. We have also modified the Newton-Rhapson iteration methods, shown in light blue in Figure 2.5, to accommodate multiple contributions to the total slip increment and plastic velocity gradient calculations.

We calibrated our proposed crystal plasticity model by focusing first on unirradiated $\alpha$-iron single crystal data and then verifying the implementation of the irradiation defect hardening models against lower-length scale simulations. In the aluminum and nickel alloy simulations covered later in this work, we relied on previously calibrated values of the CDD dislocation evolution parameters [177]. 


\section{CHAPTER 3. CONTINUUM DISLOCATION DYNAMICS MODEL FOR IRRADIATED $\alpha$-IRON}

We transition from the discussion of the theoretical foundations to the presentation of results with this chapter on the Continuum Dislocation Density (CDD) model for glide dislocations. Here we apply the CDD crystal plasticity model to a body center cubic (BCC) $\alpha$-iron material. Ferritic-martensitic irons are used in light water reactor (LWR) nuclear power plants for the reactor pressure vessel (RPV) because of their resistance to irradiation damage. Nonetheless, as discussed in Chapter 1, these $\mathrm{BCC}$ irons still experience significant irradiation damage under prolonged irradiation

doses. The primary irradiation damage defect is self-interstitial atom (SIA) loops [167]. Several groups have focused on the problem of modeling the interaction of dislocations and SIA loops in BCC metals, as we discussed in Section 1.2, yet none have fully captured the physical interactions among the key microstructure components, particularly cross-slipped dislocations. To focus on capturing the physical interactions among dislocations and SIA loops in this work we have performed simulations with $\alpha$-iron exclusively.

We explore, in particular, the modeling of the stochastic process of cross slip and the role of interaction between anisotropy in slip system strength and stochastic cross slip. Through these interactions, our CDD crystal plasticity model is able to capture 
both the stress response and the physical evolution of dislocation on different slip system planes.

In this chapter we present a dislocation density based continuum crystal plasticity model composed of physically-based interaction mechanisms in the dislocation evolution equations, including a combined stochastic-Monte Carlo approach for cross slip calculations to capture the random nature of this dislocation mechanism. This dislocation evolution model is coupled to an equation for SIA loop evolution, and hardening is calculated as a function of both dislocation and SIA interactions. Single crystal verification experiments are used to calibrate the continuum dislocation dynamics (CDD) crystal plasticity model before assessing the predictive capability of the model with a simplified polycrystalline geometry. In Section 3.1 we introduce the constitutive model and the details of the dislocation and SIA loop evolution equations. In Section 3.2 we describe our calibration of the CDD crystal plasticity model to single crystal $\alpha$-iron tensile experiments. In Sections 3.3 and 3.4 we present the results of the finite element simulations, including a discussion of cross slip approaches and a comparison to lower length scale simulations and experimental polycrystalline data, before some concluding remarks in the final section. 


\subsection{CDD Crystal Plasticity Implementation for BCC $\alpha$-Iron}

\subsubsection{Anisotroic Slip System Strength}

Physically-based frameworks write the constitutive slip system resistance equation as a function of dislocation and defect densities within the crystal [135, 161]; both defects and other dislocations act as barriers to dislocation motion [65, 204]. In this model the resistance of the slip systems to dislocation motion is considered as the sum of the physical barriers to dislocation motion, including dislocation forests and irradiation defects.

$$
g^{(\alpha)}=g_{o}^{(\alpha)}+g_{\text {forest }}^{(\alpha)}+g_{\text {irradiation-defects }}^{(\alpha)}
$$

In Eq 3.1 the Peierl's strength, or the internal lattice friction, is represented by a constant, $g_{o}^{\alpha}$. The effect of anisotropy is accounted for by adjusting the initial slip system resistance for each type of slip system type:

$$
g_{o}^{(\alpha)}= \begin{cases}2.5 \cdot \tau_{p s} & g_{o}^{\{110\} \text { type }} \\ 1.0 \cdot \tau_{p s} & g_{o}^{\{112\} \text { type }} \\ 2.0 \cdot \tau_{p s} & g_{o}^{\{123\} \text { type }}\end{cases}
$$

where $\tau_{p s}$ is the isotropic Peierls strength of the material. In light of the studies which show only the $\{110\}$ and $\{112\}$ planes are active at lower temperature [154], we follow the approach of [4] and consider only these two slip systems in our crystal plasticity 
model for a total of 24 slip systems.

\subsubsection{Stochastic Cross Slip Models}

Cross slip is a significant component of dislocation motion in BCC materials [152]. In lower length scale models, such as dislocation dynamics, the probability of cross slip from single slip systems within a cross slip family is calculated as a stochastic process. [158] presented a probability for a single dislocation to cross slip based on applied shear stress and temperature. The challenge in continuum level models, including crystal plasticity, is how to adapt the discrete probability model for continuum dislocation density values while retaining the physical basis of the cross slip model.

This work explores the effect of two different models for stochastic cross slip representation within a continuum framework: a stochastic model and a stochasticMonte Carlo combination model for calculating dislocation cross slip. The stochastic only model is taken from [145] and will be denoted in this work as stochastic-PM after the two authors; the combination stochastic and Monte Carlo method, introduced in this work, is given the notation stochastic-MC. We begin first with a description of the stochastic-MC approach. 


\section{Stochastic-MC Cross Slip Approach}

In the stochastic-Monte Carlo combination approach to modeling dislocation cross slip, we calculate the probability of cross slip with the same equation used by [158]

$$
P^{\alpha \beta}=\exp \left(\frac{-\left(\tau^{*}-\left|\tau^{\beta}\right| V_{a}\right)}{k T}\right)
$$

where $\tau^{*}$ a critical stress for a dislocation to cross slip in $\mathrm{mm}, \tau^{(\beta)}$ is the applied shear stress on slip system $\beta$ in $\mathrm{mm}, V_{a}$ is the volume $\left(\mathrm{mm}^{3}\right)$ required for cross slipped dislocation, $k$ is the Boltzmann constant, and $T$ is the temperature in Kelvin. The probability of cross slip to the slip system is calculated for each slip system within a cross slip family. The cross slip systems with the higher applied stress will have the larger probability of receiving cross slipped dislocations. We then construct a continuous distribution function (CDF) for each cross slip system by normalizing the probability by the sum of all probabilities within the slip system, such that the CDF is bounded between 0 and 1. The CDF function consists of bins for the normalized probability that a certain system will receive cross slipped dislocations.

We then perform a Monte Carlo type analysis to determine which slip system gives cross slip dislocations to which other system by comparing a random number to the CDF for the cross slip family. For each slip system, a random number from $(0,1]$ is generated, and that random number is compared against the bins in the CDF. The bin in which the random number falls is considered to be the slip system which 
receives the cross slip dislocations from the slip system for which the random number is generated.

$$
\rho_{\text {cross-slipped }}^{(\alpha)}=\sum_{\beta=1}^{\left.\beta\right|_{\text {family }}} P^{\beta \alpha} \rho^{(\beta)}=\sum_{\beta=1}^{\left.\beta\right|_{\text {family }}} \rho_{\text {received }}^{(\beta)}-\rho_{\text {given }}^{(\alpha)}
$$

where $\left.\beta\right|_{\text {family }}$ is the total number of slip systems within a cross slip family, $P^{\beta \alpha}$ is the probability of cross slip defined in Eq $(3.3), \rho_{\text {received }}^{(\beta)}$ are cross slipped dislocations received from other slip systems in the same cross slip family, and $\rho_{\text {given }}^{(\alpha)}$ is the amount of dislocation which cross slip from the current $(\alpha)$ system to another system. $\rho_{\text {received }}^{(\beta)}$ is only nonzero if the Monte Carlo analysis determines that the current slip system $(\alpha)$ receives cross slip dislocations from a $(\beta)$ system. If the random number generated and the CDF bin into which the random number falls are associated with the same slip system, that slip system is considered to not produce any cross slipped dislocations and $\rho_{\text {given }}^{(\alpha)}$ is zero.

\section{Stochastic-PM Cross Slip Approach}

The stochastic only cross slip approach is included in this work for comparison to the stochastic-Monte Carlo combination approach. The stochastic only model is implemented following the approach of [145]. In this approach, the probability of cross slip is calculated using Eq (3.3). The probability values are used as coefficients 
to the mobile dislocation densities to calculate the cross slip dislocation density.

$$
\rho_{\text {cross-slipped }}^{(\alpha)}=P^{\alpha \beta} \sum_{\beta \neq \alpha}^{\left.\beta\right|_{\text {family }}} \rho_{\text {mobile }}^{(\beta)}-\rho_{\text {mobile }}^{(\alpha)} \cdot\left(\sum_{\beta \neq \alpha}^{\left.\beta\right|_{\text {family }}} P^{\alpha \beta}\right)
$$

where the superscript $\alpha$ represents the current slip system, and the superscript $\beta$ represents the other slip systems in the same cross slip family, and $\left.\beta\right|_{\text {family }}$ is the total number of slip systems within a cross slip family.

In the stochastic-PM approach, all slip systems participate in cross slip in every iteration, loosing and gaining cross slipped dislocations. The net change of cross slipped dislocations for a single slip system depends both on the value of the probability of cross slip and on the value of the mobile dislocation densities.

\subsection{Single Crystal $\alpha$-Iron CDD Simulations}

We calibrated our proposed crystal plasticity model by focusing first on unirradiated $\alpha$-iron single crystal data and then verifying the implementation of the irradiation defect hardening models against lower-length scale simulations. 
Table 3.1: Parameter values used in the single crystal CDD $\alpha$-Fe simulations.

\begin{tabular}{|c|c|c|}
\hline Parameter & Value & Description \\
\hline$C_{11}$ & $242 \times 10^{3} \mathrm{MPa}$ & Elastic constant, [65] \\
\hline$C_{12}$ & $150 \times 10^{3} \mathrm{MPa}$ & Elastic constant, [65] \\
\hline$C_{44}$ & $112 \times 10^{3} \mathrm{MPa}$ & Elastic constant, [65] \\
\hline$\mu$ & $80 \times 10^{3} \mathrm{MPa}$ & Shear modulus, [65] \\
\hline$b$ & $2.48 \times 10^{-7} \mathrm{~mm}$ & Burgers vector, [65], Eq (2.59) \\
\hline$\dot{\gamma}_{o}$ & $4.0 \times 10^{-2}$ & Reference strain rate, Eq (2.65) \\
\hline$m$ & 0.012 & Strain rate sensitivity exponent, Eq (2.65) \\
\hline$\alpha_{m b h}$ & 0.4 & Dispersed barrier coefficient, Eq (2.71) \\
\hline$\Omega^{\alpha \alpha}$ & 1.0 & Self-hardening coefficient, Eq (2.71) \\
\hline$\Omega^{\alpha \beta}$ & 0.2 & Latent-hardening coefficient, Eq (2.71) \\
\hline$\omega^{\alpha \alpha}, \omega^{\alpha \beta}$ & 1.0 & Mean free glide path, Eq (2.69) \\
\hline$R_{c}$ & $15 b \mathrm{~mm}$ & Annihilation radius of capture, Table 2.4 \\
\hline$\tau^{*}$ & $4 \times 10^{-3} \cdot \mu$ & Critical cross slip stress, Eq (3.3) \\
\hline$V_{a}$ & $20 b^{3}$ & Volume for dislocation cross slip, Eq (3.3) \\
\hline$k$ & $1.38065 \times 10^{-20}$ & Bolztmann constant, Eq (3.3) \\
\hline$T$ & $298 \mathrm{~K}$ & Temperature, Eq (3.3) \\
\hline
\end{tabular}




\subsubsection{Unirradiated Dislocation Evolution Calibration}

Using single crystal tension test experimental data [78], we calibrated the parameters for the dislocation evolution models, Eqs 2.67 and 2.68 and Table2.4 against $\alpha$-iron single crystal data from three different loading directions, following [99]. These particular loading orientations change in the influence of the cross slip term $\left(\alpha_{5}\right.$ in Eq 2.67 and Table 2.4) by varying the number of active slip systems: [001] has four active

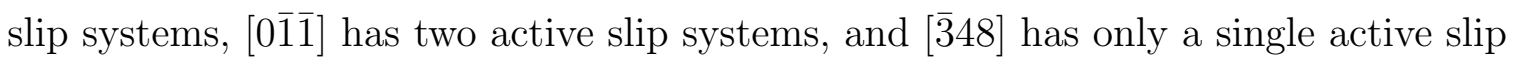
system.

The single crystal calibration simulations were performed on a $1 \mathrm{~mm}^{3}$ cube mesh consisting of 216 Hex8 elements. Symmetric boundary conditions were used and a displacement loading rate corresponding to a strain rate of $3.3 \times 10^{-4} \mathrm{~s}^{-1}$ was applied; the strain rate matches that used by [78]. The values of the elastic properties, glide velocity, and dislocation cross slip were held constant through out this calibration process and are listed in Table 3.1. We assumed equal values for the initial mobile dislocation density and the initial immobile dislocation density, set at $2.5 \times 10^{5} \mathrm{~mm}^{-2}$ [99].

Beginning with values for the dislocation evolution equation $\alpha$ parameters, Eqs (2.67) (2.68) and Table 2.4 suggested by dislocation dynamics simulations [101], we varied the values of the parameters to obtain agreement with the experimental curves 


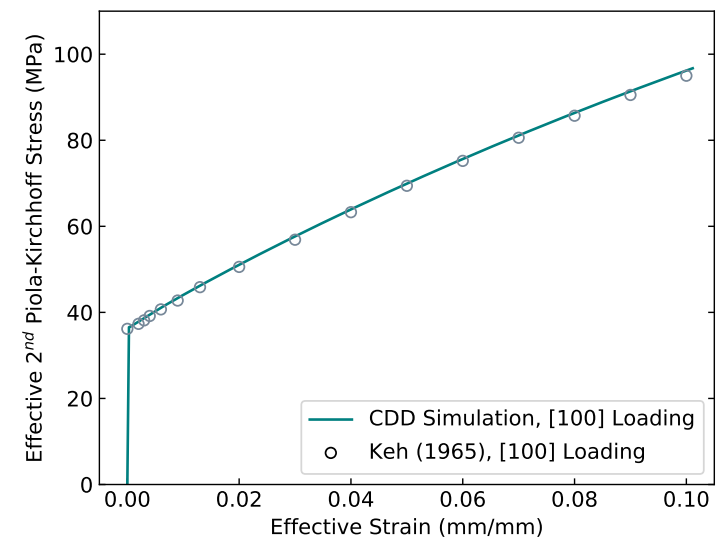

(a) $\alpha$-Fe [100] direction

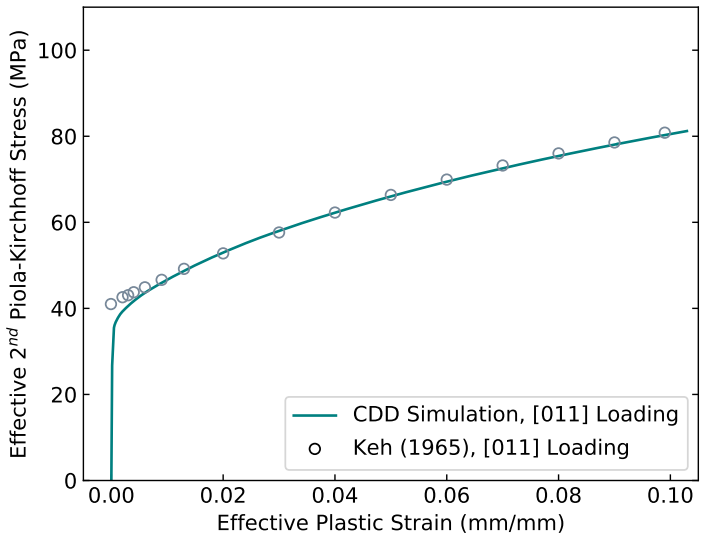

(b) $\alpha$-Fe $[0 \overline{1} \overline{1}]$ direction

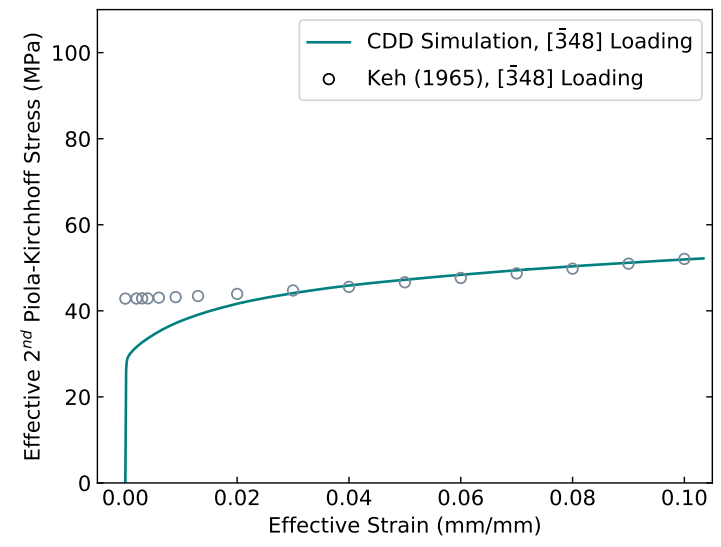

(c) $\alpha$-Fe $[\overline{3} 48]$ direction

Figure 3.1: Verification of the dislocation evolution parameters listed in Table 3.2 against single crystal BCC $\alpha$-Fe tensile experimental data [78], reproduced from [99].

for each loading orientation. The crystal plasticity simulations using the finalized parameters values are presented in Figure 3.1 against the experimental data from [78]; the specific values of the dislocation evolution parameters are listed in Table 3.2. 
Table 3.2: Dislocation evolution parameters for Eqs (2.67) and (2.68) and Table 2.4 calibrated for the crystal plasticity model in single crystal $\alpha$-Fe simulations.

\begin{tabular}{cccccccc}
\hline Load Orientation & $\alpha_{\mathbf{1}}$ & $\alpha_{\mathbf{2}}$ & $\alpha_{\mathbf{3}}$ & $\alpha_{\mathbf{4}}$ & $\alpha_{\mathbf{5}}$ & $\alpha_{\mathbf{6}}$ & $\tau_{\mathbf{p s}}$ \\
\hline$[100]$ & 0.03 & 0.5 & 0.002 & 0.002 & 0.015 & 1.0 & $15 \mathrm{MPa}$ \\
{$[0 \overline{1} \overline{1}]$} & 0.03 & 0.5 & 0.002 & 0.002 & 0.0335 & 1.0 & $8.8 \mathrm{MPa}$ \\
{$[\overline{3} 48]$} & 0.03 & 0.5 & 0.002 & 0.002 & 0.044 & 1.0 & $8.8 \mathrm{MPa}$ \\
\hline
\end{tabular}

We acknowledge the difference in the Peierls stress value used in each of the three loading directions, as was observed by [177]. The variance of the cross slip evolution parameter is an indication of the more significant role of cross slip in the deformation of the single active slip system loading direction than in the loading direction with multiple active slip systems.

\subsubsection{Mesh Convergence Study}

In conjunction with the calibration procedure for the dislocation evolution parameters, we performed a mesh convergence study on each of the three loading directions. The parameters given in Tables 3.1 and 3.2 were used in these mesh convergence studies, and the same symmetry and tensile loading boundary conditions as used in 3.2.1. These mesh convergence studies were performed using the stochastic cross slip model, 
Eq 3.4. A series of meshes, in which the number of elements in each linear direction was successively increased, were used to run the same crystal plasticity simulation: 8 $\left(2^{3}\right), 64\left(4^{3}\right), 216\left(6^{3}\right)$, and $512\left(8^{3}\right)$ total elements in the $1 \mathrm{~mm}^{3}$ cube. The results of this mesh convergence study are shown in Figure 3.2.

We observe a mesh size dependence in the results which is correlated with the number of activated slip systems: the random nature of the stochastic cross slip method impacted the mesh density required to obtain a solution in the single slip system loading direction [348] more than it did in the multiple active slip system loading direction of [100]. The [100] loading direction, with four active slips systems, displays negligible dependence on the relative element size because the cross slip has a minimal impact on the overall stress-strain curve. The simulation results for the [011] loading direction, with two active slip system, show moderate mesh dependence, with the finer mesh simulation results crossing over the coarser mesh results around 0.04 strain. The results from the single activated slip system, the [348] loading direction demonstrate significant dependence on the mesh density. With only the singly active slip system, the cross slip term plays an important role in the crystal response, and a larger number of elements is required to average the impact of the stochastic cross slip behavior. 


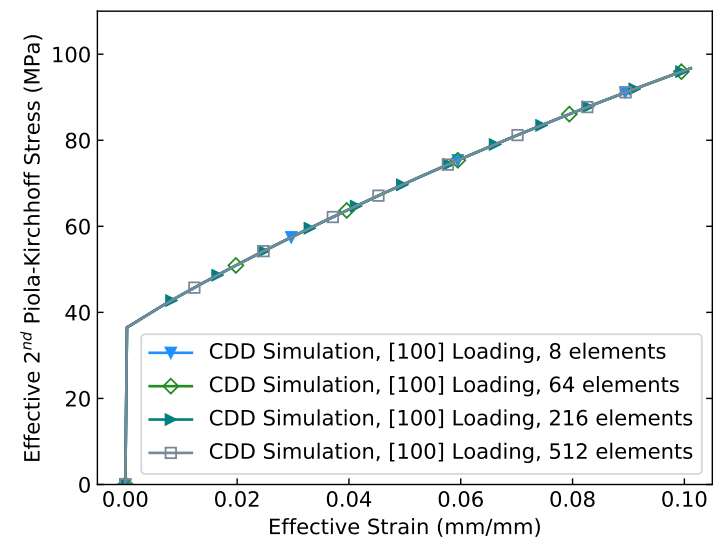

(a) [100] direction

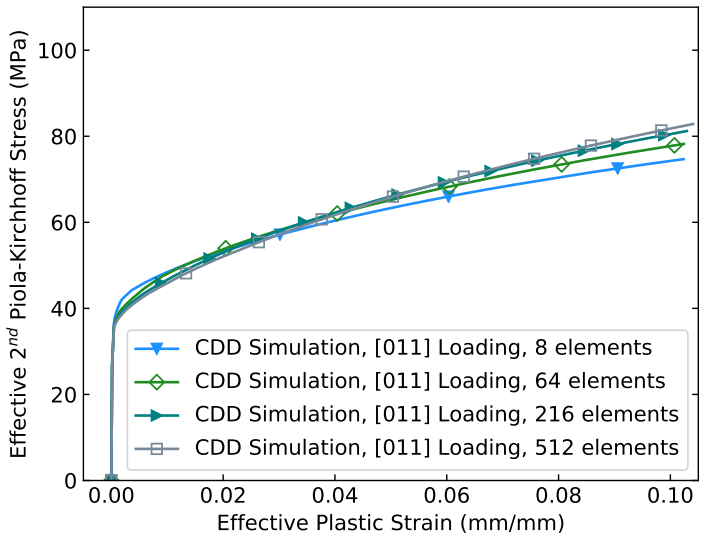

(b) $[0 \overline{1} \overline{1}]$ direction

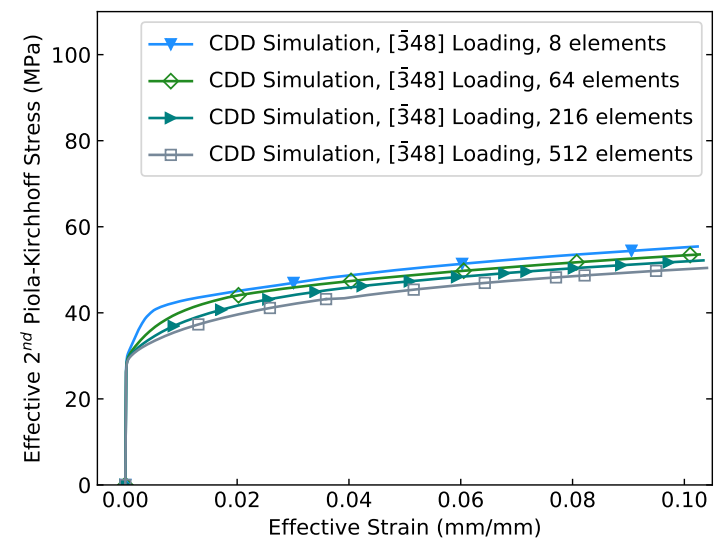

(c) $[\overline{3} 48]$ direction

Figure 3.2: Mesh convergence of the CDD crystal plasticity model depends to a degree on the number of active slip systems for the given loading direction. The loading direction with the greatest number of active slip systems, [100], demonstrated no mesh dependence while the single activated slip system loading orientation, [348], does demonstrate sensitivity to the number of elements used in the mesh. The numbers given in the legends correspond to the total number of elements in the mesh. 
Among the three mesh convergence comparisons in Figure 3.2, the stress strain response of all three loading orientations appears reasonably converged by $216\left(6^{3}\right)$ elements. Therefore, in the simulations presented in the dislocation evolution calibration, Section 3.2.1 and in the following results, Sections 3.2.4 and 3.4, we have taken the reference mesh size as 216 elements per crystal grain.

\subsubsection{Strain Rate Sensitivity}

Additionally we performed a strain rate sensitivity study, using a $1 \mathrm{~mm}^{3}$ cubic mesh geometry with the material properties from Table 3.1 and the dislocation evolution parameters for the [100] direction from Table 3.2. We applied three different tensile displacement loading conditions along the [100] direction; these displacement loading conditions correspond to strain rates of $1 \times 10^{-2} \mathrm{~s}^{-1}, 1 \times 10^{-3} \mathrm{~s}^{-1}, 1 \times 10^{-4} \mathrm{~s}^{-1}$. Symmetry boundary conditions were applied to the 216 element mesh of the single crystal. As shown in Figure 3.3, the CDD model demonstrates an expected sensitivity to the applied strain rate, with higher strain rates producing higher stress responses.

\subsubsection{Connection between Cross Slip and Anisotropy}

The inclusion of anisotropy is necessary to capture the single slip system behavior of the [348] loading direction orientation. The transfer of dislocations from the 


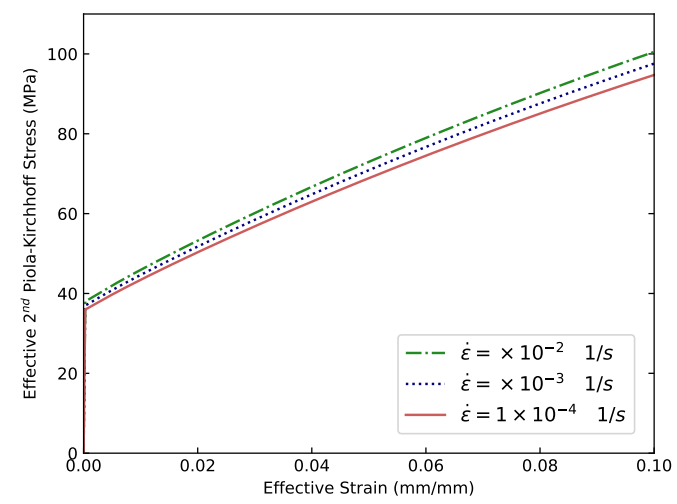

Figure 3.3: The CDD crystal plasticity model demonstrates mild sensitivity to the applied strain rate, with higher strain rates producing a higher stress response.

active $(\overline{2} 11)[111]$ system to the $(\overline{1} 01)[111]$ system and later the $(\overline{1} 10)[111]$ system relieves the dislocation growth on the active system; this dislocation growth mitigation prevents over hardening of the crystal stress response. These two systems have the highest probability of receiving cross slip dislocations within the [111] cross slip family; therefore it is likely that dislocations will cross from from $(\overline{2} 11)[111]$ to $(\overline{101)}[111]$ and (110)[111], as shown in Figure 3.4. Despite the similar probabilities of cross slip on these two systems from the similar absolute applied shear stresses, the anisotropic correction, $\mathrm{Eq}(3.2)$, to the intrinsic lattice friction value of the $\{110\}$ systems prevents activation of this slip system.

The higher slip system resistance allows the (101)[111] and (110)[111] systems to absorb the cross slipped dislocations from the $(\overline{2} 11)[111]$ system. This absorption of cross slipped dislocations from an active system by an inactive system enables our 


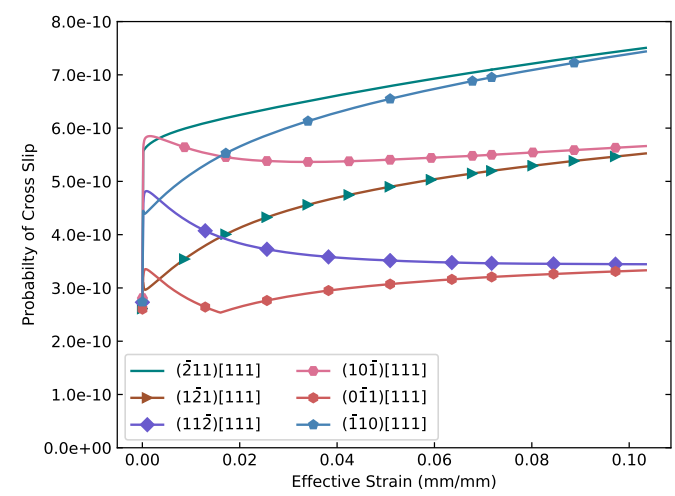

(a) Probability of cross slip among the slip systems in the [111] cross slip family

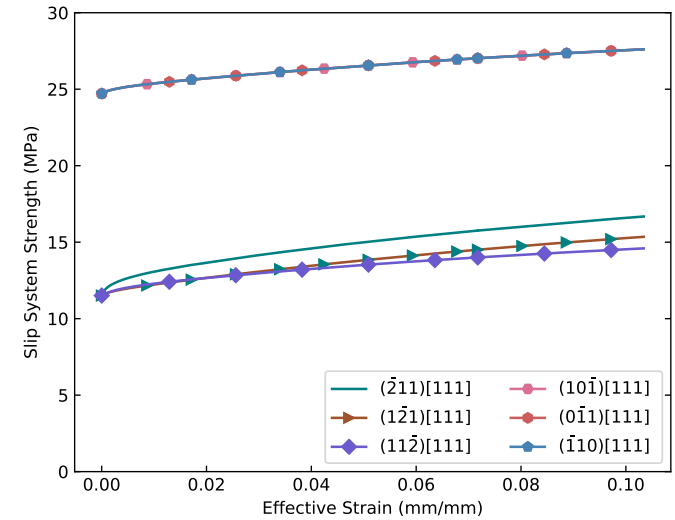

(b) Slip system strength evolution on the slip systems of the [111] cross slip family

Figure 3.4: The anisotropy correction to the intrinsic lattice friction strength, Eq (3.2), hardens the $\{110\}$ type systems relative to the $\{112\}$ type systems so that dislocations can cross slip from the lower slip resistance systems to the higher resistance systems without additional slip system activation. This figure includes the six slip systems from the [111] cross slip family.

crystal plasticity model to capture the nearly ideally plastic behavior of the single crystal loaded in the [348] direction.

\subsubsection{SIA Loop Evolution Verification}

The verification of the SIA loop defect model consists of comparing the stressstrain curves generated by our crystal plasticity model to those from dislocation dy- 
namics simulations [8], following the approach of [17]. As in the dislocation dynamics simulations, these crystal plasticity simulations were performed on $\mu \mathrm{m}^{3}$ cubes. These cubes were loaded in tension in the [100] direction with traction free boundary conditions on the lateral sides. A displacement loading rate equivalent to a strain rate of $100 \mathrm{~s}^{-1}$ was applied to the top of the cubes. Six different verification simulations were run, each with a different initial SIA loop density: $1.63 \times 10^{13} \mathrm{~mm}^{-3}, 8.15 \times$ $10^{12} \mathrm{~mm}^{-3}, 3.61 \times 10^{12} \mathrm{~mm}^{-3}, 1.63 \times 10^{12} \mathrm{~mm}^{-3}, 8.15 \times 10^{11} \mathrm{~mm}^{-3}$, and unirradiated. Following [17] an initial dislocation density of $2 \times 10^{-7} \mathrm{~mm}^{-2}$ was assumed, split evenly among mobile and immobile dislocations, and a Peierls stress of $80 \mathrm{MPa}$ was applied. The remaining SIA loop specific parameters are given in Table 3.3.

Table 3.3: Values of the parameters used in the CDD crystal plasticity model in the SIA loop terms, Eqs (2.73) and (2.72), for the verification of the SIA loop following [17].

\begin{tabular}{ccl}
\hline Parameter & Value & Description \\
\hline$\alpha_{\text {sia }}$ & 0.7 & Hardening coefficient, [96], Eq (2.72) \\
$\beta_{\text {sia }}$ & 1.0 & Loop annihilation coefficient, [114], Eq (2.73) \\
$R_{\text {sia }}$ & $15 b \mathrm{~mm}$ & Loop annihilation radius, [114], Eq (2.73) \\
$d_{\text {sia }}$ & $2.48 \times 10^{-5} \mathrm{~mm}$ & Average SIA loop diameter, [17] Eq (2.72) \\
\hline
\end{tabular}


The remainder of the model constants used in these verification simulations are those listed Table 3.1 as are the dislocation evolution parameters for the [100] loading direction in Table 3.2 .

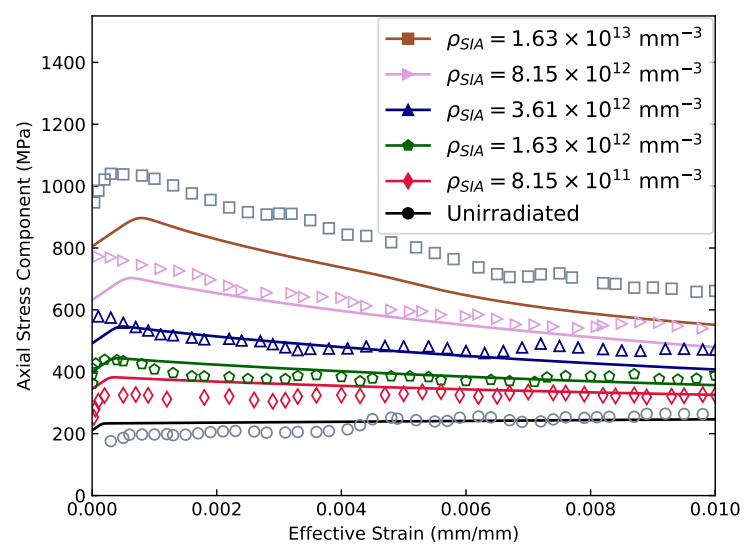

Figure 3.5: Comparison of crystal plasticity simulations with dislocation dynamics simulations of the the stress-plastic strain evolution with varying initial values of SIA loop densities demonstrate similar trends an in [17]. The dislocation dynamics data, indicated by the outlined markers, are reproduced from [8] and our crystal plasticity simulation results are shown by the solid lines.

The results of these simulations compare reasonably well with the dislocation dynamics simulations from [8], as shown in Figure 3.5. As in [17] the use of anistropic elasticity in the crystal plasticity model requires the comparison of plastic strains between the dislocation dynamics simulations and our crystal plasticity simulations.

Our model relies on a scalar form of SIA loop evolution while [17] employs a tenso- 
rial form of the SIA density rate to accounts for the 3D natures of SIA loop interaction. The 3D interaction among the SIA loops and the dislocations is accounted for with a cube root term, Eq (2.72). The comparison of this crystal plasticity model with the dislocation dynamics simulations results, Figure 3.5, demonstrates similar alignment trends with the dislocation dynamics results as shown by the more complex tensorial model of [17]. Based on these results, our scalar SIA loop density evolution rate model, Eq (2.73) is able to acceptably replicate the lower length scale results trends for varying initial SIA loop densities in a less computationally intensive manner.

\subsection{Comparison of Stochastic Cross Slip Models}

The inclusion of a cross slip term in the dislocation evolution equations is key to capturing the stress-strain behavior of the single slip loading orientation. Cross slip of dislocations away from the activated slip system mitigates the growth of the dislocation density of this slip system; thus, cross slip prevents the over hardening of the effective stress response.

A key feature of our crystal plasticity model is the inclusion of the combination stochastic-Monte Carlo cross slip term, Eq (3.4), to capture the random nature of the physical cross slip dislocation movement. The reader will recall the comparison of the mathematical theory behind the stochastic-MC approach, section 3.1.2, with 
the more commonly applied stochastic only (stochastic-PM) approach, section 3.1.2. Both cross slip approaches use the same calculation for the probability of cross slip, Eq (3.3), which is a function of the applied shear stress. The difference in the two approaches, which we investigate here, exists in the method for determining which slip systems interact in cross slip. Based on previous studies conducted with a CDD stochastic cross slip approach [101], we anticipated the largest difference in the cross slip approaches to be demonstrated in the [348] loading direction. In our comparison single crystal simulations, we apply the same symmetric boundary conditions and loading rate with the material parameters given in Table 3.1 and Table 3.2 for the [348] loading direction. In the stochastic-PM cross slip approach, we adjust the leading coefficient, $\alpha_{5}$, to produce parity in the density of the cross slipped dislocations between the two approaches at the beginning of the simulations.

With consistent trends in the probability of cross slip among slip systems, Figures $3.6 \mathrm{a}$ and $3.6 \mathrm{~b}$, we examine the impact of the two cross slip approaches on the mobile dislocation density. In both approaches, Figures 3.6c and 3.6d, the cross slip of dislocations away from the active $(\overline{2} 11)[111]$ system, and, to a lesser extent from the secondary activated $(\overline{1} 21)[11 \overline{1}]$ and $(\overline{1} 21)[11 \overline{1}]$ systems, relieve the growth of dislocations on the active slip systems. 


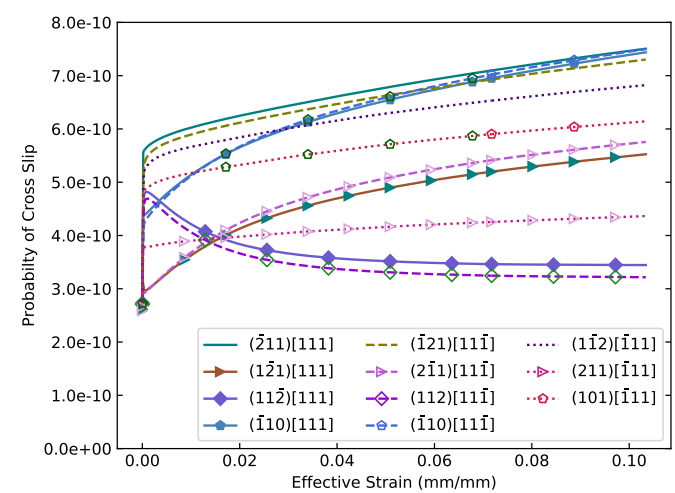

(a) Stochastic-MC: Probability cross slip

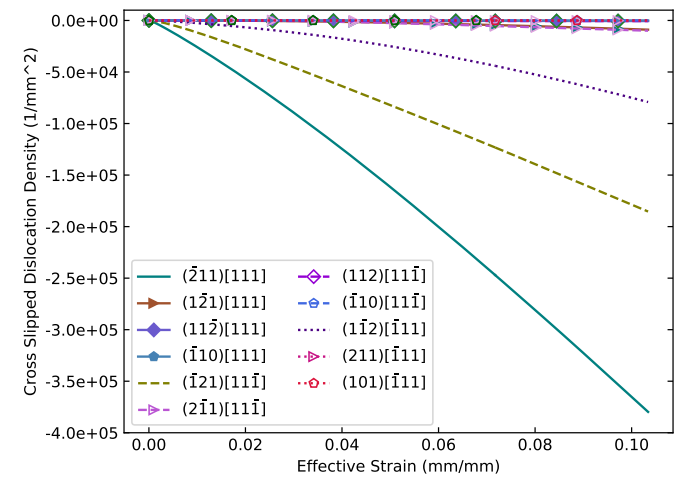

(c) Stochastic-MC: Cross slip density

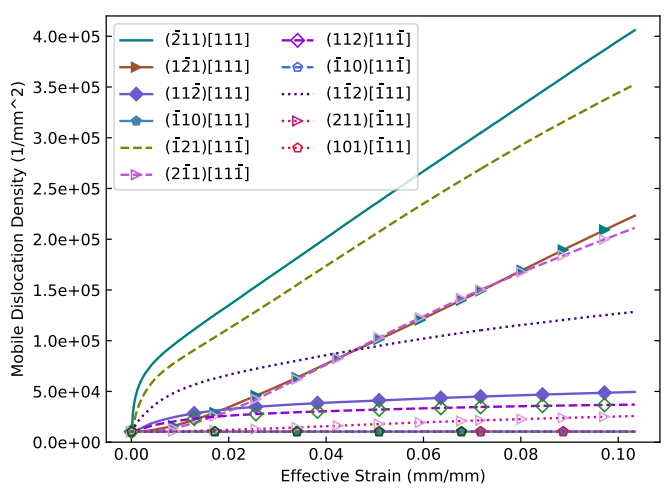

(e) Stochastic-MC: Mobile dislocations

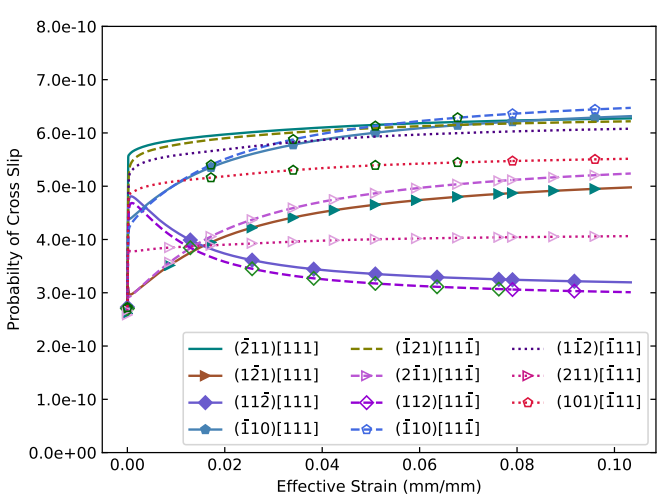

(b) Stochastic-PM: Probability cross slip

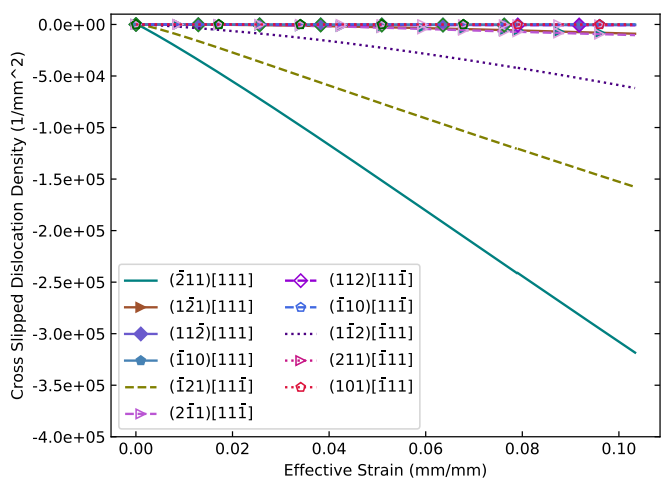

(d) Stochastic-PM: Cross slip density

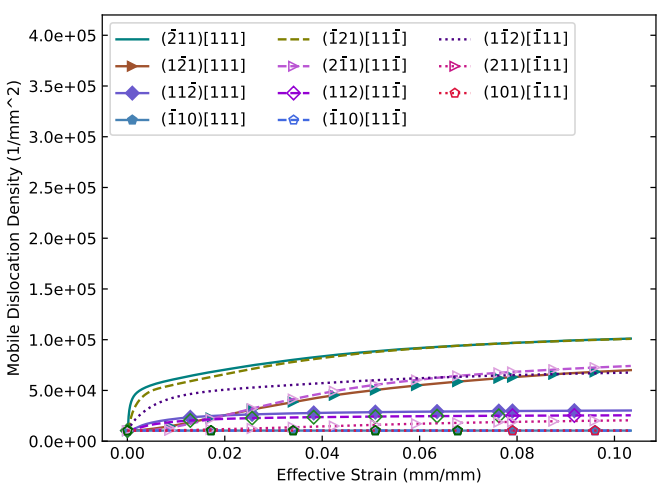

(f) Stochastic-PM: Mobile dislocations

Figure 3.6: Comparison of the cross slip approaches on the dislocation density evolution. 
In the combination stochastic-Monte Carlo approach, Figure 3.6e, this transfer of dislocations through cross slip enables the dislocation density on the active $(\overline{2} 11)[111]$ system to grow at a moderate pace. Even though the mobile dislocation density on additional slip systems $(\overline{1} 21)[11 \overline{1}]$ and $(1 \overline{1} 2)[\overline{1} 11]$ increases, the primary slip system $(\overline{2} 11)[111]$ consistently maintains the highest dislocation density value as expected in a single slip loading orientation.

In contrast, the stochastic only approach introduces an apparent saturation limit in the $(\overline{2} 11)[111]$ system mobile dislocation density. The saturation from the deterministic approach reduces the mobile dislocation density of the $(\overline{2} 11)[111]$ system to such an extent that the mobile dislocation density of the secondary $(\overline{1} 21)[11 \overline{1}]$ system grows to parity with the primary $(\overline{2} 11)[111]$ system, Figure 3.6f. While the stochastic-only approach does produce a stress-strain curve similar to the experimentally measured curve, demonstrated in Figure 3.1c, the inability of this approach to maintain the $(\overline{2} 11)[111]$ system as the primary activated slip system demonstrates the limitations of the stochastic only approach within a physically based crystal plasticity model. 


\subsection{Polycrystalline Irradiated $\alpha$-Iron Simulations}

We compare the results of our CDD crystal plasticity model to irradiated polycrystalline experimental data to evaluate the model's ability to predict RPV behavior after exposure to radiation. As a first step, we compared a polycrystalline application of the CDD crystal plasticity model to unirradiated $\alpha$-iron experimental data. Our simplified polycrystalline geometry consists of 27 equally sized cubic grains, with an average diameter of $250 \times 10^{-6} \mathrm{~mm}$ [95], and each grain is meshed with 216 elements, as shown in Figure 3.7. The orientations of the grains were determined by random assignment of the three Bunge Euler angles, within the usual angle bounds, using the Python random number generator with a normal distribution [153]. The Bunge Euler angle distribution is shown in Figure 3.8.

The dislocation evolution parameters from Table 3.2 for the [100] loading orientation were used in these polycrystalline simulations and the same $\alpha$-iron material parameters from Table 3.1, except for the Peierls stress value: we used the polycrystalline value of $11 \mathrm{MPa}$ [65]. We applied initial dislocation density value of $5 \times 10^{7}$ $\mathrm{mm}^{-2}$, which falls within the experimentally measured $7 \pm 2 \times 10^{7} \mathrm{~mm}^{-2}$ given by [95]; this value of initial dislocation density was selected by calibrating to the unirradiated data for polycrystalline $\alpha$-iron. The initial dislocation density value was split evenly among the mobile and immobile initial dislocation densities. We applied symmetry 


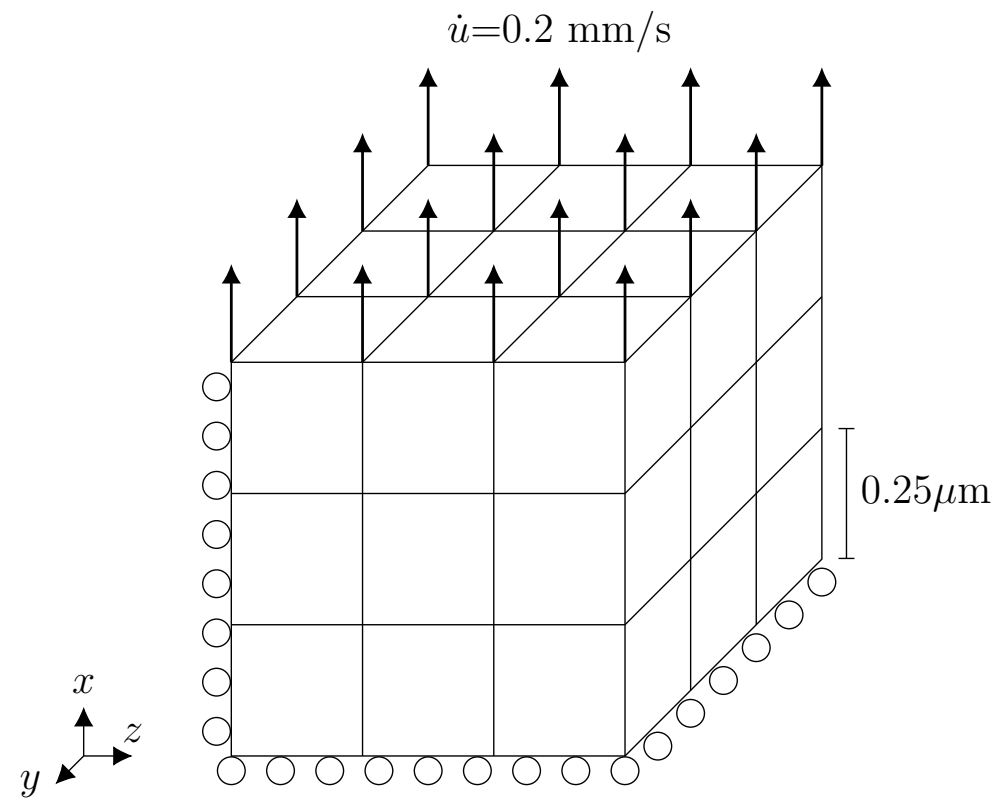

Figure 3.7: The simplified geometry, consisting of 27 cubic grains, used to examine the CDD crystal plasticity model in a polycrstalline application.

boundary condition to the model with a displacement loading rate corresponding to the strain rate of $2 \times 10^{-4} \mathrm{~s}^{-1}$ to match the experimental loading conditions, shown in Figure 3.7.

The same mesh, Bunge Euler angles, and parameters are retained for the irradiated polycrystalline simulation. To capture the effect of the irradiation defects, we included the terms for the SIA loop evolution, Eq (2.73), and interaction with the dislocations, $\mathrm{Eq}$ (2.72). The parameters for these equations, selected to correspond to an irradiation dose of $0.1 \mathrm{dpa}$, are given in Table 3.4.

We have set the value of the dislocation slip system hardening coefficient, $\alpha_{\text {sia }}$ 


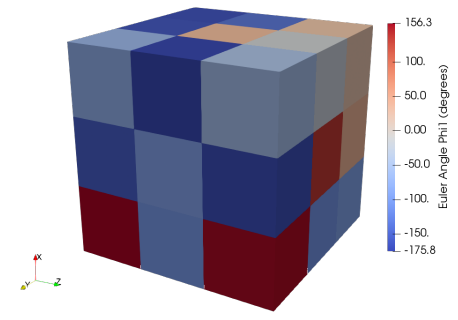

(a) Bunge Euler angle $\phi_{1}$

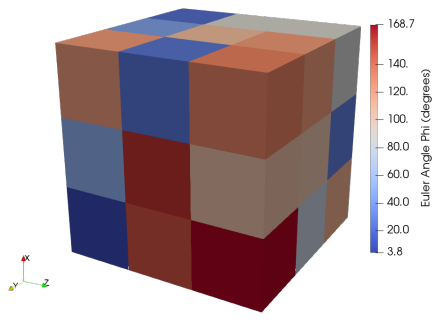

(b) Bunge Euler angle $\Phi$

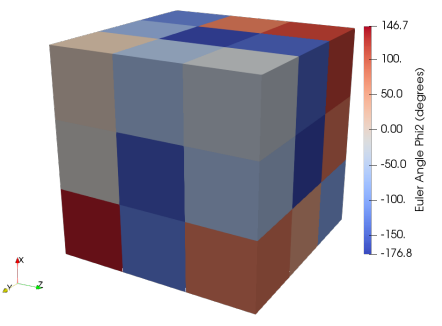

(c) Bunge Euler angle $\phi_{2}$

Figure 3.8: Crystal orientations of the 27 cubic grains in our simplified polycrystalline model, given in Bunge Euler angles, generated using the random function from the python library [153]. The orientation of these polycrystalline models matches the orientation shown in Figure 3.7.

to 0.6 , which lies between the values of 0.7 [96] and 0.37 [28] used in other studies of irradiated $\alpha$-iron. Following the approaches of $[101,28]$ we also have lowered the value of the SIA loop annihilation coefficient by a factor of 100; we hypothesize that this reduction of the SIA loop annihilation constant is a result of the much smaller loop diameter from the diameter used in the comparison to the dislocation dynamics simulations in Section 3.2.5.

The stress response of the cubic polycrstalline simulation is calculated as an effective von Mises-type stress measure, averaged across all of the quadrature points in the mesh equally. This effective second Piola-Kirchhoff stress measure is compared to the experimental data from [95] in Figure 3.9. The CDD crystal plasticity simulations 
Table 3.4: Values of the parameters used in the SIA loop terms of the crystal plasticity model for polycrystalline $\alpha$-Fe exposed to a radiation dose of $0.1 \mathrm{dpa}$, including initial conditions.

\begin{tabular}{ccl}
\hline Parameter & Value & Description \\
\hline$\alpha_{\text {sia }}$ & 0.6 & Hardening coefficient, Eq (2.72) \\
$\beta_{\text {sia }}$ & 0.01 & Loop annihilation coefficient, Eq (2.73) \\
$R_{\text {sia }}$ & $15 b \mathrm{~mm}$ & Loop annihilation radius, [114] Eq (2.73) \\
$\rho_{\text {sia }}$ & $1.2 \times 10^{12} \mathrm{~mm}^{-3}$ & Initial SIA loop number density, [119] \\
$d_{\text {sia }}$ & $7.0 \times 10^{-6} \mathrm{~mm}$ & Average SIA loop diameter, [119], Eq (2.72) \\
\hline
\end{tabular}

agree with the unirradiated experiment and the irradiated experimental data trends, Figures 3.9a and 3.9b.

The slight under prediction by the CDD simulation results of the experimentally measured data in Figures 3.9a and 3.9b indicates that the CDD crystal plasticity model lacks a hardening contribution. A possible correction to the under predicted hardening is a strain gradient type term. Such a term is often used in crystal plasticity frameworks to capture the effects of grain boundaries on the stress response of the bulk crystal [161]. We note that the build up of stress concentrations near the grain boundaries in the unirradiated simulation, Figures 3.10a and 3.10b, particularly along the boundaries of the selected grains, Figure 3.10a. The grains demonstrate 


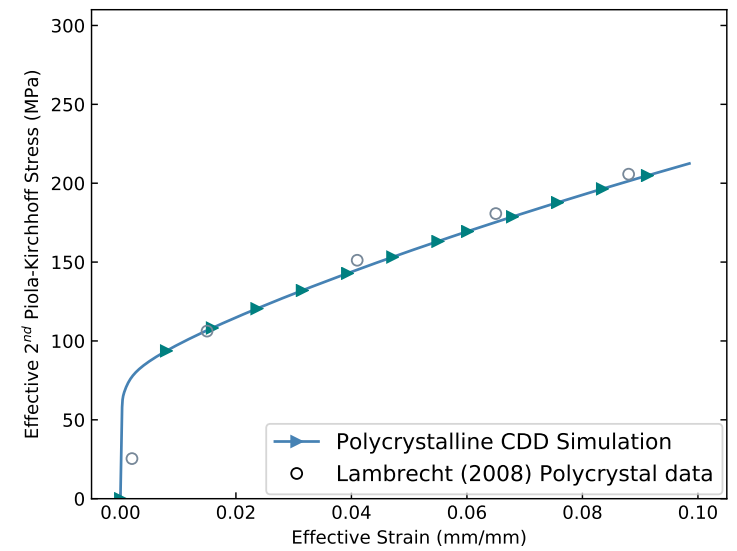

(a) Unirradiated polycrystalline $\alpha$-iron CDD simulation compared to experimentally measured tensile test data[95]

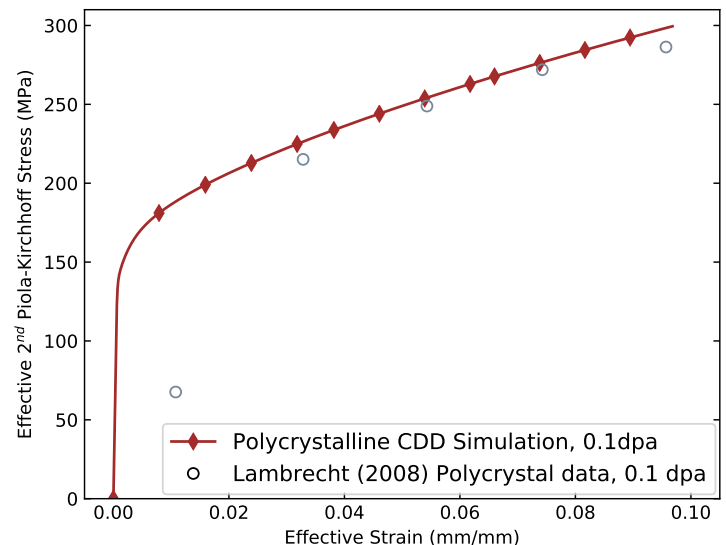

(b) Irradiated (0.1dpa dose) polycrystalline $\alpha$-iron CDD simulation compared to experimental data [95]

Figure 3.9: The CDD model captures the polycrstalline stress response of unirradiated and 0.1 dpa irradiated $\alpha$-iron with the simplified cubic polycrstalline geometry. The experimental data points for $\alpha$-iron are reproduced from [95].

an increase of stress, larger than the stress in the interior of the grains, at the grain boundaries. These stress concentrations at the grain boundaries indicate that additional hardening would be captured by the inclusion of a strain gradient term in the CDD model. 

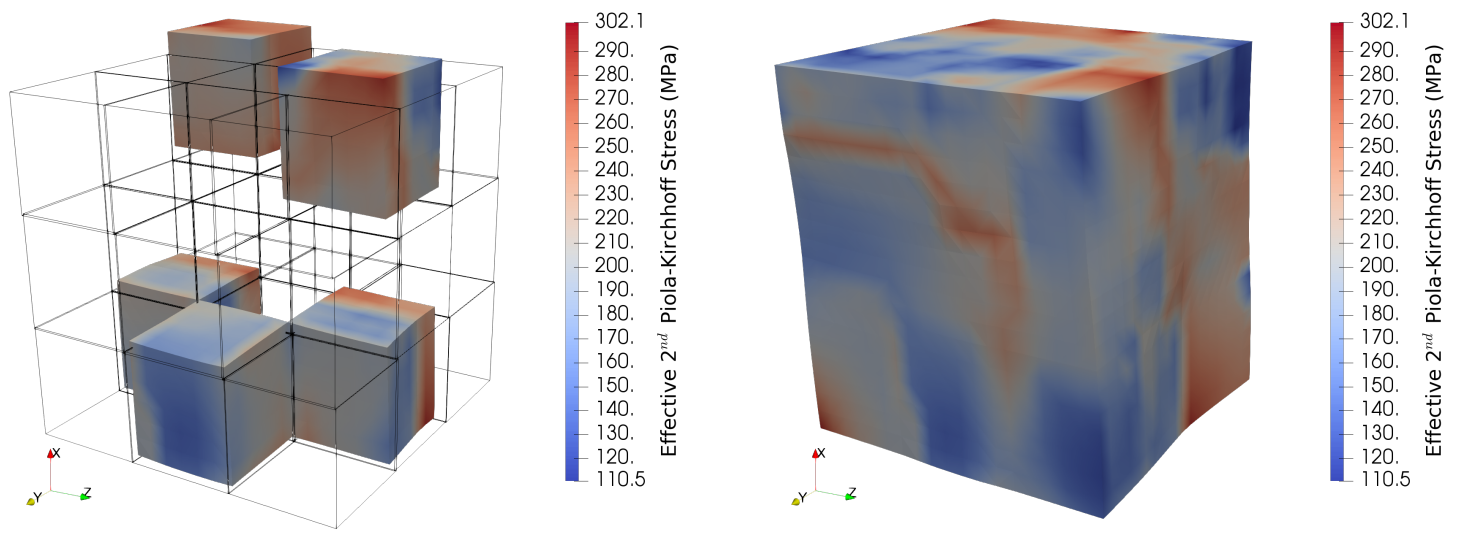

(a) Stress response of select grains within the

(b) Effective stress response of the complete simplified polystalline model from the unirradiated $\alpha$-iron case. iron at 0.1 effective strain.

Figure 3.10: An examination of the elements near the grain boundaries in the simplified cubic polycrystalline model of $\alpha$-iron reveals a buildup of stress along some boundaries of the grains, with a higher effective stress value along certain boundaries than within the remainder of the grain. The inner boundary of the top grain and the bottom boundary of the lower grain in (a) demonstrate this observed disparity among the inner grain and the grain boundaries.

The influence of the grain boundaries on the solution is also evident in the distribution of mobile dislocations along the grain boundaries. Figures 3.11a and 3.11b show the distribution of mobile dislocations on the [112](111) slip system. The inclusion of a strain gradient type term would improve the CDD crystal plasticity framework 
prediction of stresses and mobile dislocations along the grain boundary by capturing the influence of differently oriented grains. The addition of such a grain boundary sensitive term is a future development goal for the CDD crystal plasticity framework.

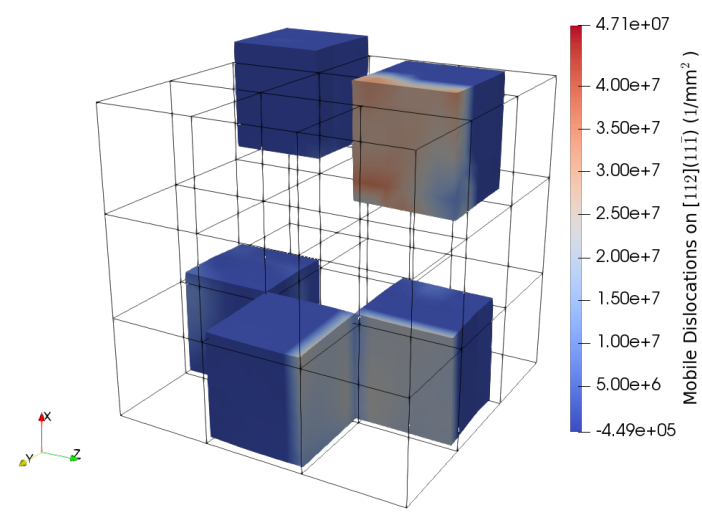

(a) Mobile dislocation density on the $[112](11 \overline{1})$ slip system of select grains within the simplified polystalline model from the unirradiated $\alpha$-iron case.

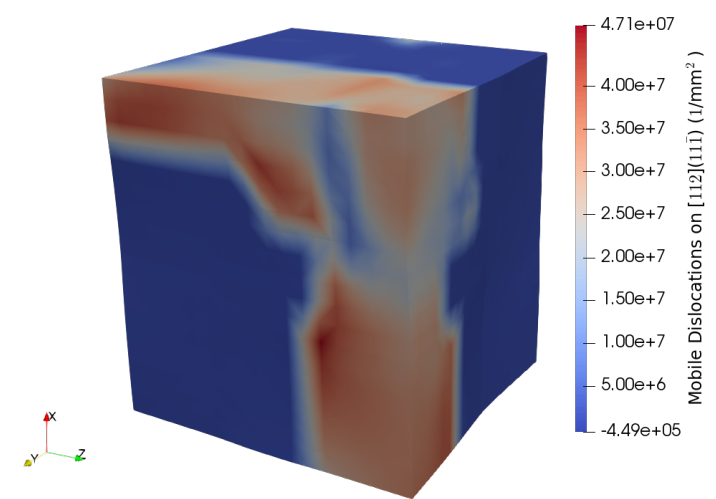

(b) Mobile dislocation density on the $[112](11 \overline{1})$ slip system in the complete polycrystalline simulation of unirradiated $\alpha$-iron at 0.1 effective strain.

Figure 3.11: The distribution of the mobile dislocations on the [112](111) slip system shows the influence neighboring grains on dislocation evolution even in the local CDD model. These results are shown on the unirradiated $\alpha$ iron simplified polycrstalline geometry.

The general alignment of our CDD model with the measured polycrystalline response, even when applied to a simplistic cubic geometry, demonstrates the capability of our crystal plasticity model to predict the hardening behavior of $\alpha$-iron exposed 
to irradiation. The physically based dislocation and dislocation-SIA loop interaction terms of the CDD crystal plasticity model can successfully capture the complex physical mechanisms of irradiated $\alpha$-iron.

\subsection{Conclusions and Future Recommendations}

We have developed a continuum dislocation dynamics crystal plasticity model with dislocation evolution terms based in physical dislocation interaction mechanisms. The dislocation evolution is coupled with SIA loop evolution in acknowledgement of the interstitial loops significant impact on the irradiation behavior of RPV steels. This model leverages the results of lower length scale molecular dynamics and dislocation dynamics simulations to establish the evolution equations for both the dislocations and the SIA loops. We calibrated the dislocation evolution components of the crystal plasticity model against single crystal tensile experiments of $\alpha$-iron. The importance of the stochastic dislocation cross slip model in conjugation with the anisotropic strength of the BCC slip systems is emphasized as necessary to correctly capture the dislocation behavior in a loading orientation selected for single slip system activation. Verification of the coupling between the dislocation evolution and the SIA loop evolution was perfomed by comparing the simulation trends from this model with trends from dislocation dynamics simulations. We then applied the CDD crystal plasticity 
model to a polycrystalline application with a simplistic geometry. The CDD model demonstrates the ability to predict the stress response of irradiated polycrystalline $\alpha$ iron, although we note that a future development goal is the addition of a strain gradient term to capture hardening contributions from the grain boundaries in polycrystalline simulations. The results of this mechanism based CDD crystal plasticity model can be used to inform engineering scale models that rely on the stress response from an evolving microstructure under radiation and deformation conditions. 


\section{CHAPTER 4. GEOMETRICALLY NECESSARY DISLOCATION DENSITY MODEL ADDITION}

Geometrically necessary dislocations form in regions of lattice bending caused by different strain patterns [68] and in low-angle grain boundaries [109]. The geometrically necessary dislocations are used within crystal plasticity frameworks to model additional resistance to mobile dislocation glide [12, 166, 56, 174, 45, 60, 124, 161, 59], even though the approaches vary significantly in the methodology and hardening models used. A number of different approaches to calculate the GND density from the Nye's dislocation tensor have been proposed and employed by different research groups, as discussed by [36]. These approaches vary from an effective GND density [50, 202], separation out into GND densities on each slip system plane [45], or categorization into screw and edge densities [108], among others. Here we follow the approach of Shizawa and Zbib by calculating a total normalized GND density for all slip systems from the Nye's dislocation tensor $[166,51]$.

In this chapter we discuss the methods used the calculate the Nye's dislocation tensor, including two approaches to calculating the derivative of the plastic velocity gradient tensor, the calculation of the total GND density, and the incorporation of the GNDs into the crystal plasticity framework described in Chapter 3. 


\subsection{Calculation of the Nye's Tensor}

We calculate the Nye's dislocation density tensor as the curl of the plastic velocity gradient as

$$
\Delta \boldsymbol{\alpha} \approx \int_{t}^{t+d t} \nabla_{X} \times \mathbf{L}^{P} d t
$$

following Shizawa and Zbib $[166,106]$. As shown in that work, this implementation is themodynamically consistent in the first intermediate configuration. In our implementation we first calculate the Rank-3 tensor gradient of the plastic velocity tensor before reducing to the Rank-2 curl derivative. Given the time dependent evolution of Eq (4.1), we have implemented an incremental approximation of the form

$$
\boldsymbol{\alpha} \approx \Delta \boldsymbol{\alpha}+\boldsymbol{\alpha}_{n-1}=\left(\boldsymbol{\nabla}_{X} \times \Delta \boldsymbol{L}^{P} \cdot \Delta t\right)+\boldsymbol{\alpha}_{n-1}
$$

where $n$ indicates the current time step increment. For consistency with Eq (2.66) we calculate the derivative of the plastic velocity gradient with respect to the undeformed configuration [36]. In this work we introduce two different methods for calculating the Rank-3 gradient of the plastic velocity gradient tensor increment, $\nabla_{X} \times \Delta \boldsymbol{L}^{P} \cdot \Delta t$, for comparison of the methods and the results from the two different approaches: a method using a sum of the gradients of the shape functions, Section 4.1.1, and a second method which uses a ZZ-patch to recover the solution of the plastic velocity gradient components at the nodes before finding the gradient at the quadrature points with the same MOOSE framework infrastructure [57] used to calculate the displacement 
gradients, in Section 4.1.2.

\subsubsection{Internal Shape Function Sum Gradient Method}

The internal elemental shape function derivative methods which we have implemented to compare against the nodal patch recovery method, Section 4.1.2, collects the shape function gradients at each quadrature point, multiples those gradients by the plastic velocity gradient stored at that quadrature point, and sums the contribution from each shape function derivative. For the Hex8 elements we use in this work, there are 8 shape function contributions to sum at each of the 8 quadrature points. The gradient of the plastic velocity gradient tensor is calculated internally at each quadrature point, independent of the plastic velocity gradient tensor at the other quadrature points within the same element or neighbor elements. For this reason we term this approach the internal shape function sum method.

Collecting the shape function gradient components at each point, we multiply the gradient components by the plastic velocity gradient to construct a Rank-3 plastic velocity gradient spatial derivative tensor at each quadrature point.

$$
\boldsymbol{\nabla} \otimes \Delta \boldsymbol{L}^{P}=\sum_{i=1}^{8} \nabla_{r} \phi_{i} \otimes \Delta L_{m n}^{P}
$$

where $\phi_{i}$ represents the eight shape functions for the Hex8 elements used in this work while $r, m$, and $n$ are traditional Newton indices. From the Rank-3 gradient of the 
plastic velocity gradient, Eq (4.3) we apply the permutation tensor to find the curl, described in Section 4.1.3 to calculate the Nye's tensor.

These shape function gradients $\nabla_{r} \phi_{i}$, are the same as those used by the MOOSE framework to calculate the gradient of the displacement values at the quadrature points. From this perspective the internal shape function sum method is straightforward to implement because it utilizes the existing FEM infrastructure. Information from the surrounding elements is not used the internal shape function sum method; thus this method needs to only query information at a single quadrature point to perform the plastic velocity gradient derivative calculation. This method is therefore more computationally efficient than the nodal patch recovery method, Section 4.1.2. Additionally, unlike the nodal patch recovery method, the internal shape function sum method allows the calculation of the GND density at the same point within the simulation timestep whereas the nodal patch recovery method results in a lag in the GND density calculation.

The downside to restraining the calculation to only the local quadrature point information is that the resulting measure of lattice curvature will be calculated on a point-wise basis and will be solely local. Geometrically necessary dislocations are often implemented to introduce the effect of non-local lattice curvature into a crystal plasticity simulation $[7,161]$. In this internal shape function method, we rely entirely on the shape functions to introduce continuity beyond the individual quadrature 
point; on the $\mathrm{C}^{0}$ Hex8 elements we have used in our simulations, this continuity is provided only across quadrature points within the same element. Furthermore, unlike the approach taken by Busso et al. and Meissoner et al., we have not implemented specialized internal second derivatives of the shape functions at the quadrature points $[24,118]$. The first derivatives of the shape functions we have used in this method are intended for finding the derivatives of nodal quantities at the quadrature points. Despite this significant shortcoming, we retain the internal shape function sum method implementation results to contrast with the results from the nodal patch recovery method.

\subsubsection{Nodal Patch Recovery (ZZ-Patch) Gradient Method}

MOOSE includes a nodal patch recovery mechanism, currently under development, which was implemented following the ZZ-patch algorithm [208]. This functionality enables the recovery of quadrature point values, such as the stress, at the nodes: a patch of quadrature points within the elements surrounding the node of interest is used to fit the solution for a value of interest at the node [208]. In this method we employ the nodal patch recovery functionality to determine a value for all nine components of the plasticity velocity gradient at the element nodes.

Each component of the plasticity gradient increment, given here as $C$, is recovery 
at a node based on the patch of quadrature points in the elements touching the node of interest, shown in Figure 4.1. The recovered component is given as an expansion

$$
C_{p}=P \cdot a
$$

where $C_{p}$ is the patch recovered component of the plastic velocity gradient, $P$ is the array of polynomial factors corresponding to the dimension and recovery element shape function term, and $a$ is the array of unknown coefficients. By minimizing the

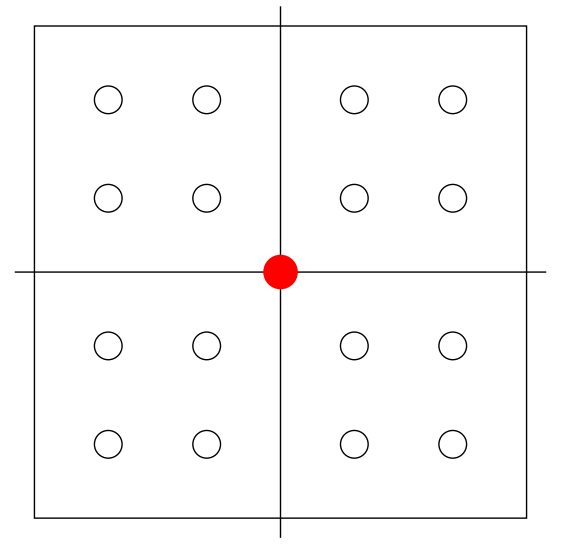

Figure 4.1: A midplane 2D representation of the bottom layer of the Hex8 elements and the quadrature points (in outline) used to recover the plasticity velocity gradient at the central node of interest (in red).

error between the directly computed component values $C$ and the estimated patch $C_{p}$ values, Zienkiewicz and Zhu determined the minimization condition

$$
a=A^{-1} \cdot b
$$


in which the definitions given below are used:

$$
\begin{aligned}
A & =\sum_{i=1}^{n} P^{T}\left(x_{i}, y_{i}, z_{i}\right) \cdot P\left(x_{i}, y_{i}, z_{i}\right) \\
b & =\sum_{i=1}^{n} P^{T}\left(x_{i}, y_{i}, z_{i}\right) \cdot C\left(x_{i}, y_{i}, z_{i}\right)
\end{aligned}
$$

where $A$ has the same value for all nine components of the plastic velocity gradient [208]. The MOOSE implementation uses singular value decomposition for Eq (4.5).

The nodal patch recovery method provides a reasonable and feasible compromise between the practically of widespread $\mathrm{C}^{0} \mathrm{FEM}$ implementations and the requirement for the GND representation of lattice curvature to depend on values beyond a single element. The nodal patch recovery method produces a nonlocal measure of the plastic velocity gradient derivative by relying on the values from all of the quadrature points in point-neighbor elements; for the node of interest shown in Figure 4.1, all of the quadrature point from the 8 elements sharing the node of interest are used in the recovery calculation. Furthermore this dependence on neighboring element values provides the resulting GND density calculation with a measure of mesh independence because the solution is not bound within a single element.

The computational costs associated with this method are a consequence of the methods in which the internal stateful material properties are reinitialized for the nodal patch recovery call; the computational cost is not a consequence of the actual recovery algorithm iteself $[208,147]$. The use of the stateful plastic velocity gradient from neighboring elements poses a challenge when running simulations in parallel: 
these values are not generally retrievable across processors. Additional functionality to ghost the set of internal stateful properties on elements bordering processor divisions has been added as a temporary fix to MOOSE [147]. This additional ghosting of information also contributes to the non-negligible slowdown of the GND calculations using the nodal patch recovery method.

The nodal patch recovery algorithm is run at the end of each nonlinear iteration step in the simulation. Since the algorithm, Eqs (4.4) and (4.5), is run once for each component of the plastic velocity gradient, it must be called a total of nine times per node in the mesh at the end of each nonlinear iteration. The algorithm could also be called at the end of the timestep iteration, but we have noted more computational efficiency in calling the algorithm at the end of each nonlinear iterations. In this way the value of the GND density lags the dislocation glide slip increment calculation by only a single nonlinear iteration rather than by an entire timestep.

\section{Rank-3 Gradient of Recovered Plastic Velocity Gradient}

Once the nine plastic velocity gradient tensor components have been recovered at the nodes, the same element shape functions used to calculate the displacement gradients are multiplied with the plastic velocity gradient tensor components. This treatment is identical to the approach used within the MOOSE framework to calculate the gradient of any nodal field variable. This multiplication creates a Rank-3 gradient of the plastic velocity gradient tensor at each of the quadrature points. Unlike the 
local Rank-3 gradient tensor calculated by the internal shape function sum method, Section 4.1.1, the nodal patch recovery method applies the shape function gradients to the nodal recovered plastic velocity components, $\Delta L_{p}^{P}$.

$$
\nabla \otimes \Delta \boldsymbol{L}_{p}^{P}=\nabla_{r} \phi \otimes \Delta L_{p}^{P}
$$

Furthermore, because the nodal patch recovery method employs the MOOSE framework infrastructure to calculate the nodal variable gradient, this method does not directly sum the contributions from the shape functions.

The Rank-3 tensor, Eq (4.8) is then used to calculate the Nye's tensor using the curl definition discussed by Das et al [36]. The approach to reduce the Rank-3 derivative to the Rank-2 curl is the same for both the internal shape function sum method and the nodal patch recovery method and is discussed in Section 4.1.3.

\subsubsection{Calculation of the Curl of the Plastic Velocity Gradient}

Having calculated the Rank-3 derivative of the plastic velocity gradient tensor with one of the two methods described in Sections 4.1.1 and 4.1.2, we now reduce the gradient to the Rank-2 curl with the permutation tensor. As mentioned by Das et al., several works calculate the curl of a strain measure to compute the Nye's dislocation tensor, with several inconsistencies in the varied approaches [36, 171]. Das et al. describe three different formulations for calculating the curl, all presented 
in a manner so as to be consistent with each other. Two of these formulations have the same order as the definition of the Nye's dislocation tensor we employ in this work, Eq (4.1),

$$
\begin{aligned}
& (\boldsymbol{\nabla} \times \boldsymbol{P})_{k m}=\epsilon_{i j k} P_{j m, i} \\
& (\boldsymbol{\nabla} \times \boldsymbol{V})_{k m}=\epsilon_{i j k} P_{m j, i}
\end{aligned}
$$

and it follows that these two formulation are equivalent when $\left(P_{j m}\right)^{T}=V_{m j}$. Following the commonly used conventions for cyrstal plasticity FEM calculations of the Nye's dislocation tensor [7, 27], we employ the definition of the curl in Eq (4.10).

Das et al. and others [35, 108], define the dislocation density tensor as

$$
\boldsymbol{\alpha}=\left(\nabla_{X} \times \boldsymbol{F}^{P}\right)^{T}=\left(\epsilon_{i j k} F_{m j, i}^{P}\right)^{T}
$$

showing that the transpose follows from the application of Stoke's theorem to the equation for the Burgers closure failure around GNDs [36].

To relate the plastic deformation gradient used in Eq (4.11) to the plastic velocity gradient used in Eq (4.1), we recall from Chapter 2 the definition of the deformation gradient, $\mathrm{Eq}$ (2.8). In the case of the plastic deformation gradient the change in displacement term is related to the sum of the slips due to dislocation glide on all the slip systems [42]:

$$
\boldsymbol{F}^{P}=\boldsymbol{I}+\boldsymbol{\beta}=\boldsymbol{I}+\sum_{\alpha} \gamma \hat{\boldsymbol{s}} \otimes \hat{\boldsymbol{m}}
$$


where $\boldsymbol{I}$ is the Rank-2 identity tensor and $\boldsymbol{\beta}$ is the crystallographic slip tensor equal to the sum of the slips [41]. Noting that the rate form of second term in Eq (4.12) is by definition the plastic velocity gradient in our crystal plasticity formulation, Eq (2.64) we rewrite Eq (4.11) as a function of the plastic velocity gradient

$$
\dot{\boldsymbol{\alpha}} \approx\left(\boldsymbol{\nabla}_{X} \times\left(\boldsymbol{I}+\boldsymbol{L}^{P}\right)\right)^{T}=\left(\nabla_{X} \times \boldsymbol{L}^{P}\right)^{T}
$$

since the gradient of the identity tensor is zero. Thus our definition of the Nye's dislocation tensor is:

$$
\dot{\alpha}_{k m} \approx \epsilon_{i j m} L_{k j, i}^{P}
$$

Written out directly as it is implemented in the code, the Nye's dislocation tensor is computed at each quadrature point as

$$
\dot{\boldsymbol{\alpha}} \approx\left[\begin{array}{lll}
\frac{\partial L_{x z}^{P}}{\partial X_{y}}-\frac{\partial L_{x y}^{P}}{\partial X_{z}} & \frac{\partial L_{x x}^{P}}{\partial X_{z}}-\frac{\partial L_{x z}^{P}}{\partial X_{x}} & \frac{\partial L_{x y}^{P}}{\partial X_{x}}-\frac{\partial L_{x x}^{P}}{\partial X_{y}} \\
\frac{\partial L_{y z}^{P}}{\partial X_{y}}-\frac{\partial L_{y y}^{P}}{\partial X_{z}} & \frac{\partial L_{y x}^{P}}{\partial X_{z}}-\frac{\partial L_{y z}^{P}}{\partial X_{x}} & \frac{\partial L_{y y}^{P}}{\partial X_{x}}-\frac{\partial L_{y x}^{P}}{\partial X_{y}} \\
\frac{\partial L_{z z}^{P}}{\partial X_{y}}-\frac{\partial L_{z y}^{P}}{\partial X_{z}} & \frac{\partial L_{z x}^{P}}{\partial X_{z}}-\frac{\partial L_{z z}^{P}}{\partial X_{x}} & \frac{\partial L_{z y}^{P}}{\partial X_{x}}-\frac{\partial L_{z x}^{P}}{\partial X_{y}}
\end{array}\right]
$$

Recall that the individual $\boldsymbol{L}^{P}$ terms in $\mathrm{Eq}$ (4.15) are computed with one of the approaches discussed in Section 4.1.1 or Section 4.1.2.

We further note that this computation of the Nye's dislocation tensor is also equivalent to the definition given by Shizawa and Zbib:

$$
\alpha_{i j}=\epsilon_{j k l} A_{i l, k}=\epsilon_{k l j} A_{i} l, k \rightarrow \epsilon_{i j m} A_{k j, i}
$$


where $\boldsymbol{A}$ represents the rank-3 gradient of the plastic velocity gradient tensor [166]. The pattern of the indices in Eq (4.16) is identical to the definition of the Nye's tensor which we have implemented, Eq (4.14); thus the two approaches yield equivalent results.

\subsection{Geometrically Necessary Dislocation Density Measure}

From the Nye's tensor, Eq (4.2), we used a straightforward approach to calculate the GND density,

$$
\rho_{G N D}=\frac{c_{g}}{b} \sqrt{\alpha_{i j} \alpha_{i j}}
$$

where $\rho_{G N D}$ is the density of geometrically necessary dislocations $\left(1 / \mathrm{mm}^{2}\right), c_{g}$ is the GND density coefficient, and $b$ is the Burgers vector. This approach results in a uniform value of GND density on all slip systems [124, 106]. The GND density has units of $1 / \mathrm{mm}^{2}$, consistent with the representation of the mobile and immobile statistically stored dislocations previously discussed in Section 2.3. Our effective GND density calculation follows the use of effective measures implemented by several other researchers, both in computational efforts $[51,56,108,1]$ and experimental measurements of GNDs[44, 162]. We note that this total form of GND density is much simplified compared to other formulations which track the evolution of GND dislocations on each slip plane, by edge and screw components, or by a combination of both 
$[10,45,36,161]$

We find that our total approach, Eq (4.17), is justified both by the method in which the GND evolution is coupled to the evolution of the glide dislocations and by the inherent computational efficiency. In particular we couple the GND density to the glide dislocation evolution through a modification to the mean free glide path, $\mathrm{Eq}(2.69)$

$$
l_{i n v}^{(\alpha)}=\beta_{\text {path }} \sqrt{\sum_{\beta} \omega^{(\alpha \beta)}\left(\rho_{\text {mobile }}^{(\beta)}+\rho_{\text {immobile }}^{(\beta)}\right)+c_{g}^{*} \rho_{G N D}}
$$

where $c_{g}^{*}$ is a fitting parameter, as done by other groups $[138,102]$. We employ this coupling to avoid artificially over-hardening the system, a concern which has been raised about other methods of hardening the slip systems[124], with direct GND contributions to the slip system strengths as a component of forest hardening by modifying Eq (2.71). Furthermore, as noted by Ma et al., a unique solution does not exist for GND populations separated onto individual slip systems at the boundary of two adjoining crystals[109]. Since one of the objectives of this work is to study the impact of grain boundaries on the mechanical response of polycrystalline metals, this limitation is significant. Utilizing the effective total GND density, Eq (4.17) for all slip systems avoids this conundrum.

Applying our modification solely to the mean free glide path length to account for hardening due to GNDs, the integration of our GND capability into the CDD crystal plasticity model framework is straightforward. We continue to use the same mobile 
and immobile dislocation densities, the statistically stored dislocations, as presented in Section 2.3. With this coupled model we have introduced the ability to capture a measure of non local effects within the CDD crystal plasticity framework.

\subsection{Thin Single Crystal Beam Bending Benchmark Problem}

We begin our presentation of the results from our GND enabled CDD crystal plasticity model with a set of smaller simulations. In benchmarking our solution against the results from problems defined in literature, we ensure that our implementation is physically sound. In this section we will discuss the results of our GND implementation in a single crystal beam bending simulation.

For our first GND benchmark simulation we have selected a thin beam bending problem. The beam problem allows us to inspect the evolution of GND density in two different curvatures: an expansion of the lattice on the tension side of the neutral axis and a contraction of the lattice on the compression side of the beam. The thin depth of the beam allows us to examine the change of GND density along the width of the beam while reducing the computational load that would occur with a thick beam simulation. Given the total measure of our GND density calculation, Eq (4.17), we expect to have equal amounts of GND densities on both edges of the beam width and a minimum of GNDs in the center of the beam at the neutral axis. 
We use this benchmark problem both to verify the calculation of the expected GND density pattern and to explore the effect of the mesh size on the calculated GND density solution. We have run this beam bending problem with both the internal shape function sum method, Section 4.1.1, and the nodal patch recovery method, Section 4.1.2, to calculate the plastic velocity gradient derivative. From Eq (4.14), Eq (4.2), and Eq (4.17) the GND density determination is directly impacted by the plastic velocity gradient derivative calculation method.

\subsubsection{Thin Beam Geometry and Boundary Conditions}

We have modeled a thin beam of nickel in a similar fashion to the problem constructed by Ohashi to examine the evolution of GND density [136]. Our thin beam is a $0.2 \mathrm{~mm}$ by $0.1 \mathrm{~mm}$ nickel plate, with a bending displacement of $\pm 1.0 \times 10^{-5} \mathrm{~mm}$, applied over a 1 second duration [136], as illustrated in Figure 4.2.

The single nickel crystal is oriented such that the (11) $)$ plane normal lies along the z-axis and the [101] direction is parallel to the x-axis. The Bunge Euler angles used to achieve this orientation are $\phi_{1}=60^{\circ}, \Phi=125.3^{\circ}$, and $\phi_{2}=45^{\circ}$. The displacement

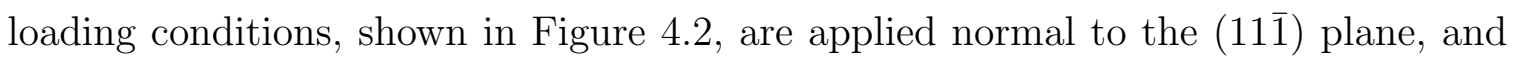
the base of the beam is fixed in all three directions. 


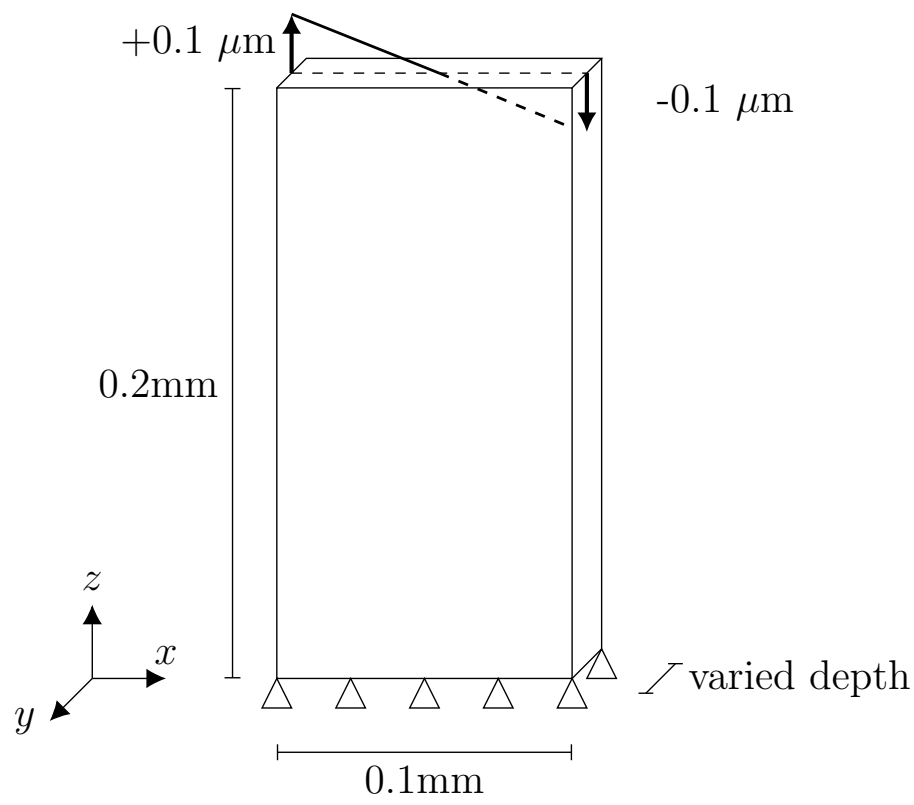

Figure 4.2: A representation of the thin beam benchmark geometry used to evaluate the evolution of GND density in the CDD crystal plasticity model, with displacement boundary conditions shown at the top of the beam to introduce a bending motion.

Table 4.1: The set of varied $y$ dimension depths of the thin beam, Figure 4.2, where the length of the cubic Hex8 elements is determined by the beam depth. The corresponding number of elements in the $x$ direction width and in the $z$ direction height are also given.

\begin{tabular}{ccc} 
Varied Depth $(\mathbf{m m})$ & Elements in Width & Elements in Height \\
\hline 0.01 & 10 & 20 \\
0.0667 & 15 & 30 \\
0.005 & 20 & 40 \\
0.004 & 25 & 50
\end{tabular}


In this convergence study we varied the depth of the beam, in the $y$ direction, along with the number of elements in the beam width and height, $x$ and $z$ directions, to ensure the use of cubic Hex 8 elements with equal length edges. In all of the simulations performed in this section we maintain a single element thickness through the beam depth, as done by [136]. To maintain cubic Hex8 elements, we vary the depth dimension of the beam, indicated in Figure 4.2; thus a finer mesh will have a thinner depth. The set of depth dimensions and the number of Hex8 elements in the width and height dimensions are listed in Table 4.1.

\section{Crystal Plasticity Model Parameters}

The elastic constants used in this set of simulations are for nickel, [65], and these values and the dislocation hardening parameters are given in Table 4.2. The CDD dislocation glide parameters are taken from a dislocation dynamics study of single crystal aluminum [177]; we have elected to use these dislocation evolution parameters because aluminum and nickel are FCC materials with higher stacking fault energies. The dislocation density evolution parameters are given in Table 4.3, and the glide slip systmes for an FCC material, Table 2.2 are used in these simulations.

In this study we have elected to remove the effects of cross slip and have set the associated dislocation evolution and cross slip material properties parameters to zero in Table 4.2 and Table 4.3. 
Table 4.2: Constant material parameter values used in the single crystal nickel simulations of the thin beam bending benchmark.

\begin{tabular}{|c|c|c|}
\hline Parameter & Value & Description \\
\hline$C_{11}$ & $246.5 \times 10^{3} \mathrm{MPa}$ & Elastic constant, [65] \\
\hline$C_{12}$ & $147.3 \times 10^{3} \mathrm{MPa}$ & Elastic constant, [65] \\
\hline$C_{44}$ & $124.7 \times 10^{3} \mathrm{MPa}$ & Elastic constant, [65] \\
\hline$\mu$ & $94.7 \times 10^{3} \mathrm{MPa}$ & Shear modulus, [65] \\
\hline$b$ & $2.48 \times 10^{-7} \mathrm{~mm}$ & Burgers vector,[65], Eq (2.59) \\
\hline$\dot{\gamma}_{o}$ & $4.0 \times 10^{-2}$ & Reference strain rate, $\mathrm{Eq}(2.65)$ \\
\hline$\alpha_{m b h}$ & 0.4 & Dispersed barrier coefficient, Eq (2.71) \\
\hline$\Omega^{\alpha \alpha}$ & 1.0 & Self-hardening coefficient, Eq (2.71) \\
\hline$\Omega^{\alpha \beta}$ & 0.2 & Latent-hardening coefficient, Eq (2.71) \\
\hline$\omega^{\alpha \alpha}, \omega^{\alpha \beta}$ & 1.0 & Mean free glide path, Eq (2.69) \\
\hline$R_{c}$ & $15 b$ & Annihilation radius of capture, Table 2.4 \\
\hline$\tau_{p s}$ & 19.47 MPa & Isotropic Peierls strength, Eq (3.2) \\
\hline
\end{tabular}


Table 4.3: Values of the dislocation evolution parameters used in the thin beam bending simulations to compare the approaches of calculating the plastic velocity gradient derivative. These parameters, for the CDD dislocation evolution model, Eq (2.67) and Eq (2.68), are appropriate for an FCC material and are slight modified from the values determined by [177] for nickel based on fitting conducted as supplemental component of this work.

\begin{tabular}{lcl}
\hline Dislocation Evolution Interaction & Constant & Value \\
\hline Frank-Read generation & $\alpha_{1}$ & 0.04 \\
Mobile-mobile annihilation & $\alpha_{2}$ & $4.0[177]$ \\
Dislocation locking & $\alpha_{3}$ & $0.002[177]$ \\
Locked dislocations freed & $\alpha_{4}$ & $0.002[177]$ \\
Cross slip & $\alpha_{5}$ & 0.0 \\
Immobile-mobile annihilation & $\alpha_{6}$ & 4.0 \\
Initial total mobile dislocation density & $\rho_{o}^{\text {mobile }}$ & $1.44 \times 10^{6} 1 / \mathrm{mm}^{2}[157]$ \\
Initial total immobile dislocation density & $\rho_{o}^{\text {immobile }}$ & $1.44 \times 10^{6} \quad 1 / \mathrm{mm}^{2}[157]$ \\
\hline
\end{tabular}

Unlike the approach taken by Ohashi [136], we allow the crystal slip systems to harden in response to dislocation density growth, as indicated by the non-zero hardening parameters in Table 4.2. Both statistically stored dislocations, directly, Eq (2.71), and geometrically necessary dislocations, indirectly, Eq (4.18) contribute to the slip system hardening. Thus we expect to see an increase in the slip system 
strengths, particularly at the beam edges, as a result of GND density growth. The effect of the GND density on the active slip system strength, in addition to the pattern of calculated GND densities, will be used to evaluate the two methods of calculating the plastic velocity gradient and thus the GND density.

\subsubsection{Thin Beam Bending Results}

In this section we distinguish between the internal shape function sum, Section 4.1.1, and the nodal patch recovery, Section 4.1.2, methods of calculating the plasticity velocity gradient derivative and thus the GND density values. We compare these two methods both in terms of convergence of the solutions. The results of the mesh convergence study are shown in Figure 4.3 for the internal shape function sum method and in Figure 4.5 for the nodal patch recovery method. In these figures the quantities of interest are sampled along the width of the beam at half the beam height in the undisplaced mesh, that is at $z=0.1 \mathrm{~mm}$, at the end of the simulations when the complete displacement loading conditions have been applied.

The value of the GND density calculated with the internal shape function sum method is essentially zero: the calculated GND density value is 18 orders of magnitude smaller than the initial glide dislocation density. This zero result is the consequence of calculating the derivative of the plastic velocity gradient within single elements. 


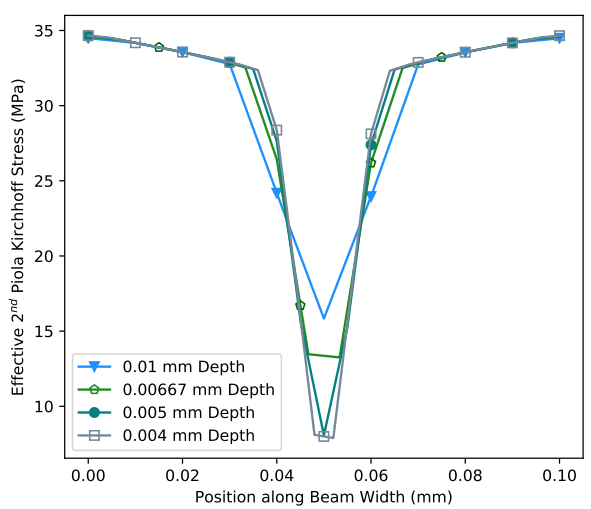

(a) Internal shape function sum method:

Effective Second Piola Kirchhoff stress variation with decreasing beam depth and increasing mesh element density

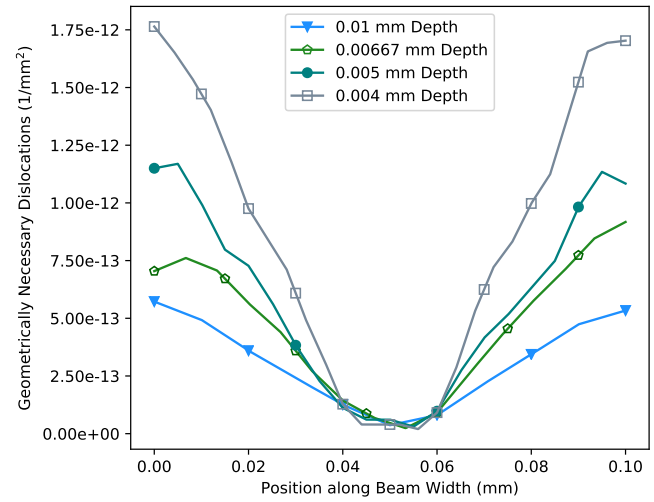

(b) Internal shape function sum method: Total GND density profile variation with decreasing beam depth and increasing mesh element density

Figure 4.3: The internal shape function sum method, discussed in Section 4.1.1, shows good convergence in the solution of the effective stress, Figure 4.3a, but only poor convergence in the solution of the GND density. The calculated GND density is numerically zero. Nonetheless, in examining the pattern of the low GND densities, we note the increasing divergence of the calculated GND density at the edges of the beam, Figure 4.3b, as an indication of the mesh dependence of this approach. These results are taken across the width of the beam at half of the beam height and correspond to the final bending load application.

Despite this result, examining the pattern of the GND density provides useful insights into the reliability of the shape function sum method. The results vary significantly with increasing number of elements in the mesh: the value of the GND density changes 
by an order of magnitude among the four different beam depths and finite element mesh considered as shown in Figure 4.3b.

The effective stress response for the internal shape function sum method is nearly as converged across the different beam depths and mesh densities, Figure 4.3a as is the effective stress response for the nodal patch recovery method, Figure 4.5a. The converged behavior of the stress occurs because the calculated GND density is approaches zero and thus the stress is not influenced by the GND density or its variation. Nonetheless the near zero GND density values and the lack of convergence in the GND density values clear demonstrate the dependence of the internal shape function sum method on the mesh and mesh size.

The mesh dependence of the internal shape function is also demonstrated in the distribution of the GND density across the entire beam face. In Figure 4.4b the GND density is shown on the $0.005 \mathrm{~mm}$ depth beam, with 800 elements, for the internal shape function method. The GND distribution from the internal shape function method resembles a patchwork solution, with only a general trend of higher GND density on the edges of the beam and generally lower GND densities near center of the beam. This voxel-type result, with significant variation in the results calculated in neighboring elements, highlights the solely local nature of this method. The lack of convergence in the GND density values clearly demonstrates the dependence of the internal shape function sum on the mesh density. 


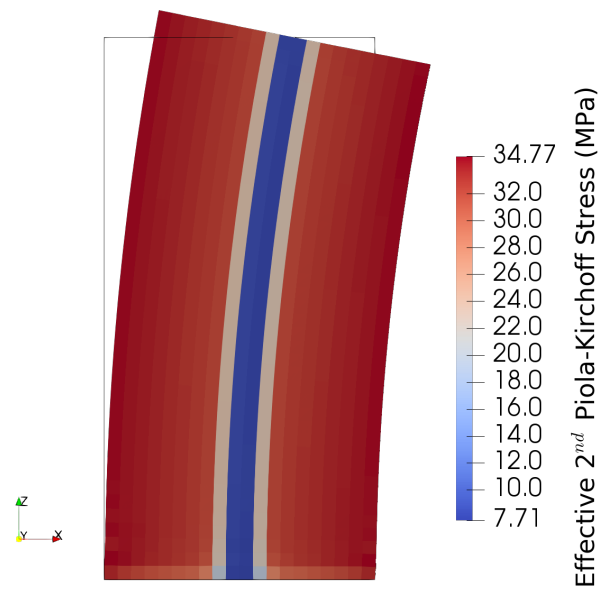

(a) Internal shape function sum method:

Effective Second Piola Kirchhoff stress

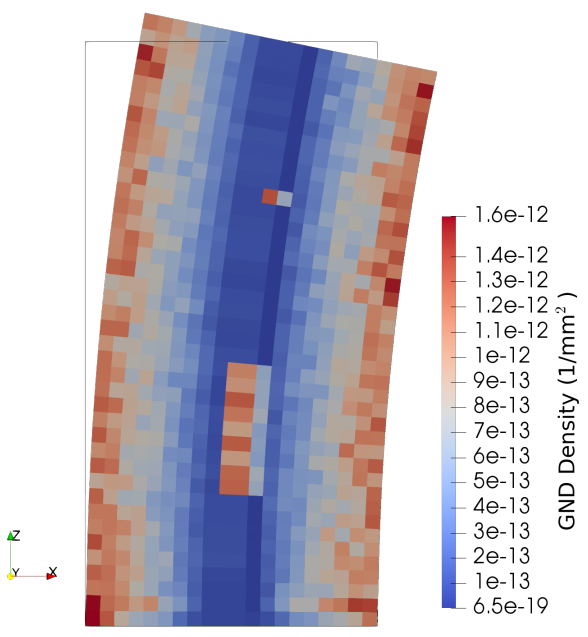

(b) Internal shape function sum method:

Distribution of the GND density

Figure 4.4: While the effective stress demonstrates the classical smooth stress distribution pattern expected in a beam bending problem, the GND density calculated with the internal shape function sum method demonstrates significant variation and pixelation. These results are shown for the $0.005 \mathrm{~mm}$ depth beam under the final bending load. The beam displacement shown in these figures has been scaled by a factor of 100 for clarity. The original beam geometry is shown in outline for reference.

In contrast the GND results from the nodal patch recovery method demonstrate both solution convergence, Figure 4.5, and a smooth distribution of the calculated solution across the beam face, Figure 4.6b. The results for the nodal patch recovery method of calculating the plastic velocity gradient derivative and the Nye's tensor 
show acceptable convergence with decreasing beam depth and increasing mesh density: the distribution of the GND density demonstrates a measure of convergence in the center of the beam and tight convergence of the solution at the beam edges, Figure 4.5b.

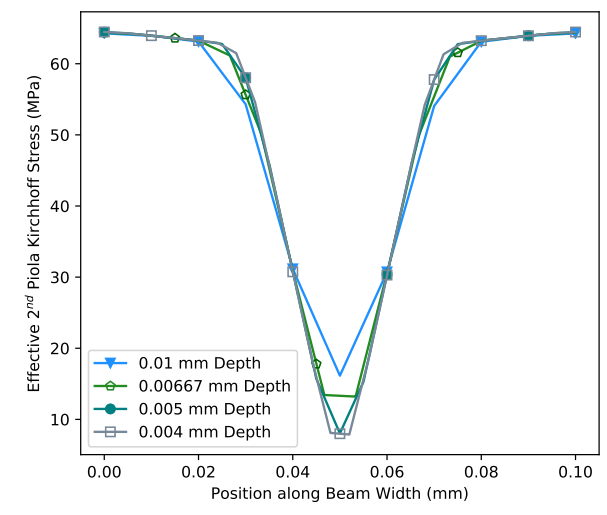

(a) Nodal patch recovery method: Effective

Second Piola Kirchhoff stress variation with decreasing beam depth

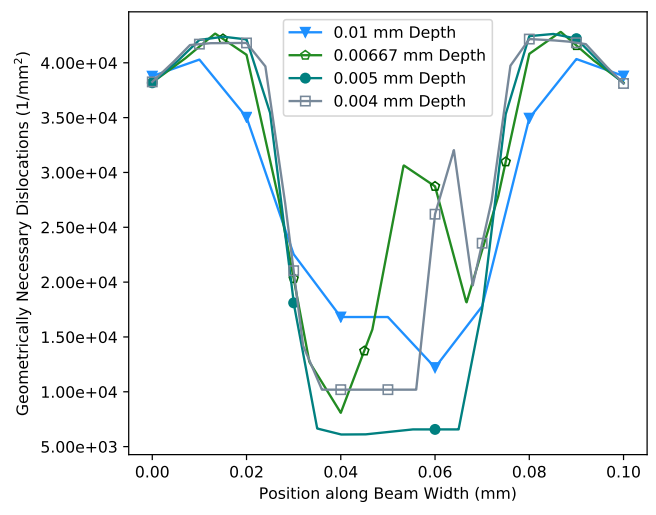

(b) Nodal patch recovery method: Total GND density profile variation with decreasing beam depth and increasing mesh density

Figure 4.5: The Nodal Patch Recovery method, discussed in Section 4.1.2, shows good convergence in the solution of the effective second Piola-Kirchhoff stress, Figure 4.5a, and acceptable convergence in the GND density at the edges of the beam, Figure 4.5b. The general convergence of the GND density calculated with this method is an indication of the measure of mesh independence provided by the nodal patch recovery method. These results are taken across the width of the beam at half of the beam height and correspond to the final bending load application. 
Unlike the pixelated results of the internal shape function sum method, Figure 4.4b, the GND results from the nodal patch recovery demonstrate smooth bands, with high GND densities calculated at the beam edges and a lower GND density value calculated at the beam neutral stress axis, Figure $4.6 \mathrm{~b}$.

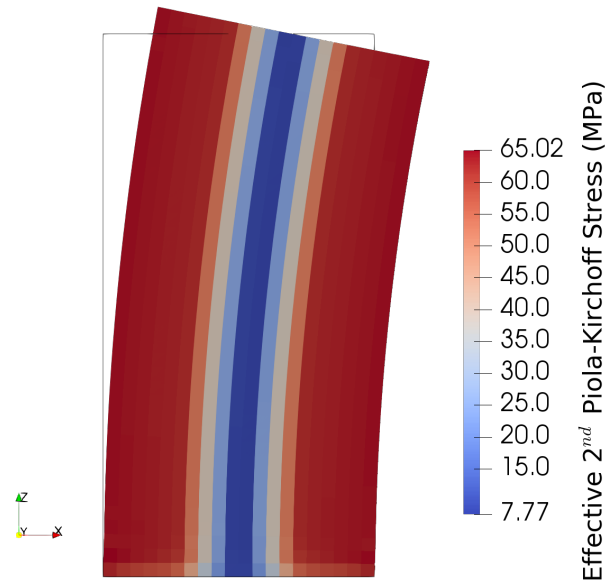

(a) Internal shape function sum method:

Profile of the effective Second Piola

Kirchhoff stress as shown on the $0.005 \mathrm{~mm}$

depth beam at complete loading.

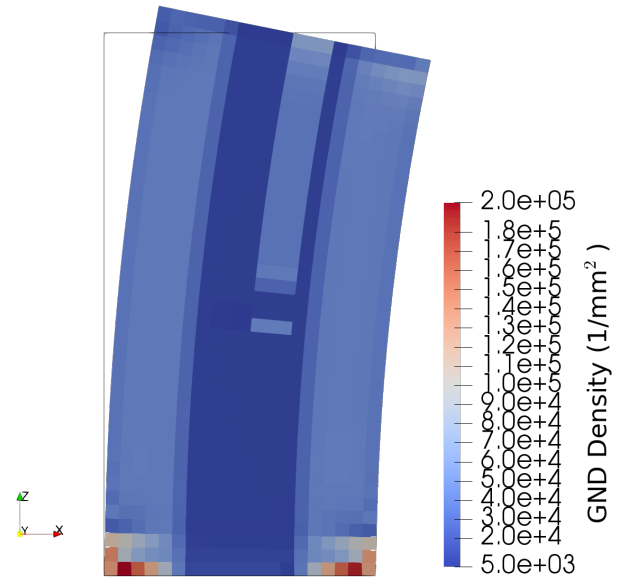

(b) Internal shape function sum method: Distribution of the GND density as shown on the $0.005 \mathrm{~mm}$ depth beam under the final bending load.

Figure 4.6: Both the effective second Piola-Kirchhoff stress and the GND density distribution demonstrate smooth distributions across the $0.005 \mathrm{~mm}$ depth beam, with the higher values for both quantities occuring on the edges of the beam as expected. The beam displacement shown in these figures has been scaled by a factor of 100 for clarity. The original beam geometry is shown in outline for reference. 
We note the variation of the GND density distribution in Figure $4.6 \mathrm{~b}$ at both the upper middle of the beam and at the lower corners of the beam. The high concentrations of GND densities at the lower corners of the beam are the effect of the fixed boundary conditions applied on the lower edge of the beam as shown in Figure 4.2. This increase in GND density was also noted by Ohashi in his original study with this benchmark problem [136]. We hypothesis that the band of increased GND density in the upper center of the beam may be a result of allowing the crystal slip systems to harden, a crystal plasticity implementation which Ohashi did not employ. Nonetheless this unexpected band warrants further investigation.

Finally, to directly compare the two methods of calculating the plastic velocity gradient derivative, $\nabla \cdot L^{P}$, and the effect of these methods on the calculated solutions in the benchmark beam bending problem, we have compared four quantities of interest from the $0.005 \mathrm{~mm}$ beam depth simulations. In addition to the effective stress response and the GND density quantities which we have compared previously, we also introduce comparisons of mobile dislocations and the slip system strength for a representative active slip system. Since the solutions of the mobile dislocation density and slip system resistance were consistently similar among six active slip systems, we have selected a single slip system, (111)[101], for use in the comparisons shown in Figure 4.7 . 


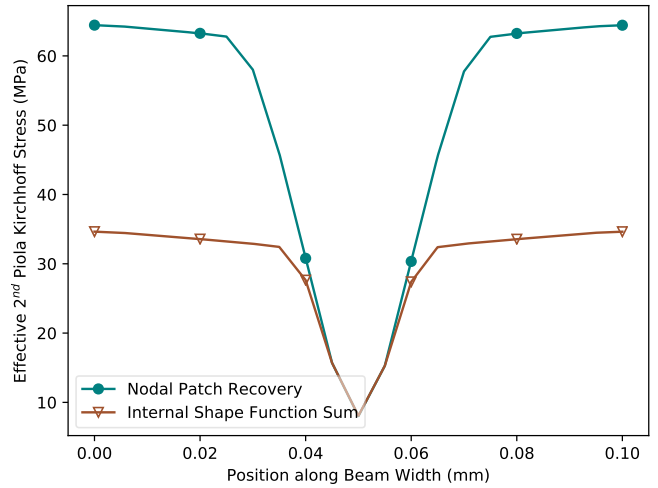

(a) Effective Second Piola Kirchhoff stress

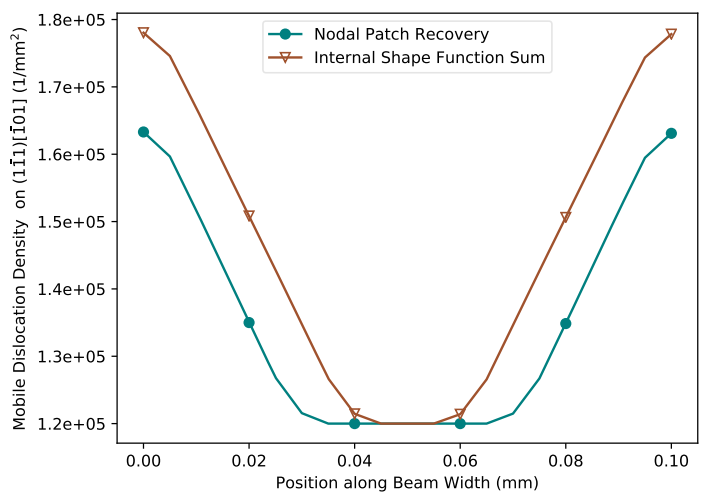

(c) Mobile dislocation density across the bent beam on the active $(1 \overline{1} 1)[\overline{1} 01]$ system

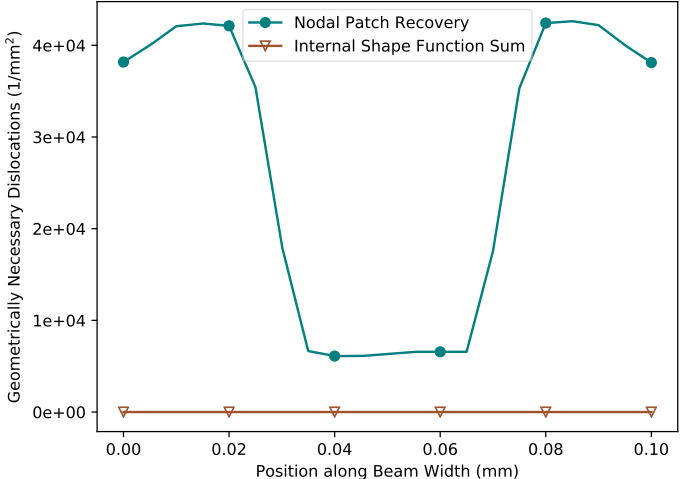

(b) Total GND density profile

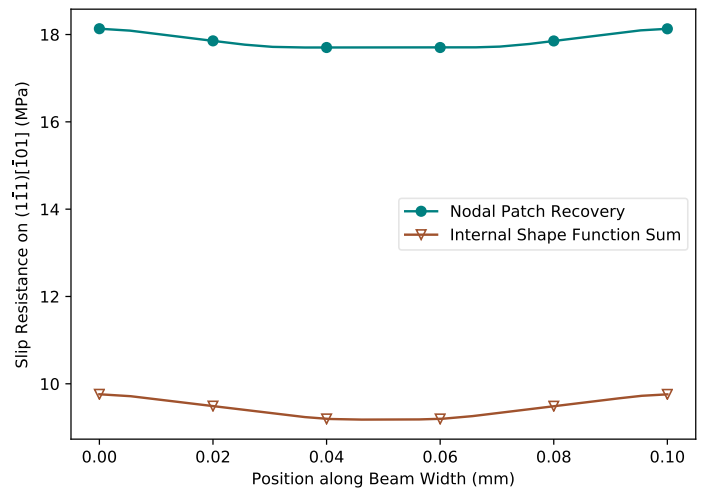

(d) Slip system strength across the bent beam on the active $(1 \overline{1} 1)[\overline{1} 01]$ system

Figure 4.7: The comparison of the Nodal Patch Recovery and Internal Shape Function Sum methods demonstrate a significant difference in calculated quantities of interest, on beams with a depth of $0.005 \mathrm{~mm}$. These results are sampled across the beam at half of the beam height under the final bending displacement. 
We note that the value of the GND density from the internal shape function method is significantly less than the GND density calculated with the nodal patch recovery method, as shown in the comparison in Figure 4.7b. Since the GND density calculated with the internal shape function sum method is several orders of magnitude smaller than the mobile dislocation density, see the mobile dislocation density from a representative active slip system in Figure 4.7c, the high variation in GND density is not propagated to the slip system strengths or to the overall stress response. The stress response for the internal shape function sum method is dominated by the mobile dislocation densities.

In Figure 4.7 the low value of GND density predicted by the internal shape function sum method results in a softer response of the crystal, with the mobile dislocations on active slip systems providing the main source of hardening. In contrast the impact of the GND density as computed with the nodal patch recovery method, through the mean free glide path, Eq (4.18), is clearly seen on the representative active slip system resistance, Figure 4.7d and on the effective stress response, Figure 4.7a.

In light of the smooth and convergent results from the nodal patch recovery method, we proceed to use only this method in the following two sections. The nodal patch recovery method predicts the expected distribution pattern and thus is a more reliable model for GND density calculation in addition to demonstrating a better measure of mesh independence. 
We do, however, note that the nodal patch recovery method requires significantly more computational time: for the $0.005 \mathrm{~mm}$ depth, 800 element mesh beam simulation the nodal patch recovery method required 306.87 hours on 4 processors to complete while the internal shape function sum method only required 1.027 hours on 6 processors. The significantly longer computation requirements of the nodal patch recovery method are a result of the reinitalization of stateful properties algorithm in the MOOSE framework, as discussed in Section 4.1.2. Reducing the computational load and the simulation runtime of the nodal patch recovery method is a priority of future work with the GND model.

\subsection{Bicrystal in Shear Simulation}

In our second benchmark problem we examine the results of the nodal patch recovery method for calculating the GND density in an aluminum bicrystal under shear loading. This bicrystal simulation, replicated from the experiment and simulation comparison conducted by Ma et al. [109] is complementary to the beam bending problem in Section 4.3.2. Where the beam bending problem used a single element depth geometry under a combination of tensile and compression loading, this bicrystal problem applies the GND model addition in a thick geometry under shear loading, completing the set of common loading conditions under which we have tested the 
GND model addition to the CDD crystal plasticity framework.

The clear focus of this benchmark problem is on the grain boundary between the two crystals. The emphasis on the relatively large grain boundary in the problem allows us to explore how the GND model addition captures the impact of the grain boundary in the overall stress response of the crystals.

\subsubsection{Bicrystal Geometry and Boundary Conditions}

The bicrystal used in this benchmark problems includes a grain boundary on the $x-z$ plane, which divides the geometry into two crystals of equal height.
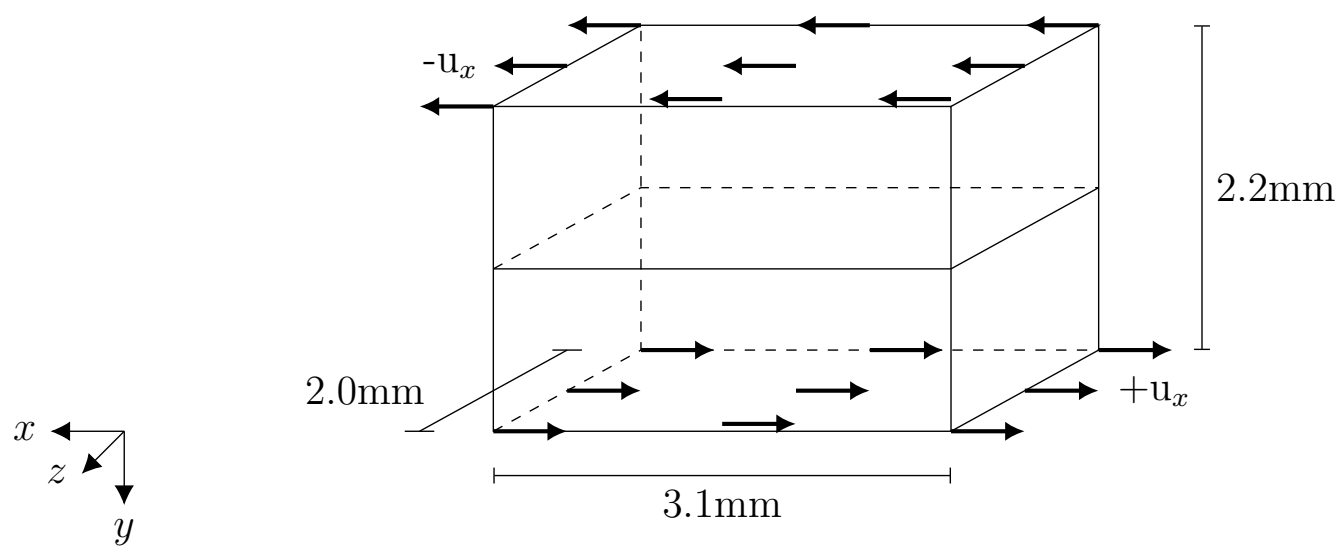

Figure 4.8: The bicrystal problem, adapted from Ma et al. [109], with the grain boundary located on the midway vertical plane. In addition to the shear loading in the $\mathrm{x}$-direction shown, the displacements in the $y$ - and z-directions are fixed on the top and bottom surfaces. 
Table 4.4: The set of Bunge Euler angles used in the set of three simulations of the bicrystal problem, taken from $[108,109]$. The geometry of the bicrystal problem is shown in Figure 4.8, and the notation top grain and bottom grain correspond to the orientation of the bicrystal as shown in the schematic.

\begin{tabular}{lcccc}
\hline Simulation & Grain Block & \multicolumn{2}{c}{ Bunge Euler Angles (degrees) } \\
& & $\phi_{1}$ & $\Phi$ & $\phi_{2}$ \\
\hline Single crystal & Top grain & 3.4 & 37.6 & 36.5 \\
& Bottom grain & 3.4 & 37.6 & 36.5 \\
& & & & 37.4 \\
Small boundary angle & Top grain & 277.0 & 32.3 & 44.3 \\
& Bottom grain & 264.7 & 32.3 & 50.1 \\
& & & & 52.9 \\
\hline
\end{tabular}

Parallel to the grain boundary on the edges of the bicrystal geometry, shear displacement boundary conditions are applied, as shown in Figure 4.8. The shear displacement rate is $\pm 4.03 \times 10^{-4} \mathrm{~mm} / \mathrm{s}$, on each face, in the $x$ direction as indicated in the schematic. The displacements in the $y$ and $z$ directions are set to zero on the same two faces on which the shear loading is applied.

Using the set of small and medium grain boundary misorientation angles defined 
by [109] we can examine the contribution of the GND model addition to the simulation of polycrystals. Nye's tensor approach is not suitable for modeling high misorientation angle boundaries [108]; thus we have elected to model only the small and medium grain boundary angle bicrystals. As done by [109] we also include a single crystal simulation. In the simulation run for the single crystal we have used the same mesh as for the two bicrystal problems, shown in the schematic in Figure 4.8, and have assigned the same grain orientation to both grain blocks, as indicated in Table 4.4. These sets of bicrystal orientations are selected in view of the observation of limitations on the Nye's tensor approach for modeling high misorientation angle boundaries [108].

\section{Crystal Plasticity Model Parameters}

The elastic constants and Burgers vector used in this set of simulations are for aluminum [65]. As in the thin beam bending simulations, Section 4.3, the CDD dislocation evolution parameters are taken from [177], which fit the CDD evolution parameters for FCC aluminum. The FCC slip systems, Table 2.2, are used in these simulations with an initial total dislocation density of $1.0 \times 10^{6} \mathrm{~mm}^{-2}$ [10], split evenly among the initial mobile and initial immobile dislocations. The set of material parameters used in the bicrystal simulations are listen in Table 4.5 and the dislocation evolution parameters are given in Table 4.6. 
Table 4.5: Constant material parameter values used in the aluminum sheared bicrystal benchmark simulations.

\begin{tabular}{|c|c|c|}
\hline Parameter & Value & Description \\
\hline$C_{11}$ & $108.2 \times 10^{3} \mathrm{MPa}$ & Elastic constant, $[65]$ \\
\hline$C_{12}$ & $61.3 \times 10^{3} \mathrm{MPa}$ & Elastic constant, [65] \\
\hline$C_{44}$ & $28.5 \times 10^{3} \mathrm{MPa}$ & Elastic constant, [65] \\
\hline$\mu$ & $26.5 \times 10^{3} \mathrm{MPa}$ & Shear modulus, [65] \\
\hline$b$ & $2.863 \times 10^{-7} \mathrm{~mm}$ & Burgers vector,[177], Eq (2.59) \\
\hline$\dot{\gamma}_{o}$ & $1.0 \times 10^{-2}$ & Reference strain rate, [177], Eq (2.65) \\
\hline$\alpha_{m b h}$ & 0.4 & Dispersed barrier coefficient, Eq (2.71) \\
\hline$\Omega^{\alpha \alpha}$ & 1.0 & Self-hardening coefficient, Eq (2.71) \\
\hline$\Omega^{\alpha \beta}$ & 0.2 & Latent-hardening coefficient, Eq (2.71) \\
\hline$\omega^{\alpha \alpha}, \omega^{\alpha \beta}$ & 1.0 & Mean free glide path, Eq (2.69) \\
\hline$R_{c}$ & $15 b \mathrm{~mm}$ & Annihilation radius of capture, Table 2.4 \\
\hline$\tau_{p s}$ & $2.65 \mathrm{MPa}$ & Isotropic Peierls strength, Eq (3.2) \\
\hline
\end{tabular}

In the following results section we present simulation results for each of the three bicrystal grain boundary orientations, Table 4.4, on a course 640 element mesh. In this coarse mesh each Hex8 element length is approximately $3 \mathrm{~mm}$. While this large mesh is not composed of ideally cubically shaped Hex8 elements, the coarser mesh allows 
us to run the bicrystal simulations to the onset of plasticity despite the significant computational load of the nodal patch recovery method as discussed in Section 4.3.2.

Table 4.6: Values of the dislocation evolution parameters used in the simulations of the sheared bicrystal, Figure 4.8. These parameters are for the CDD dislocation evolution model, Eq (2.67) and Eq (2.68) and are fit for FCC aluminum [177].

\begin{tabular}{lcl}
\hline Dislocation Evolution Interaction & Constant & Value \\
\hline Frank-Read generation & $\alpha_{1}$ & $0.02[177]$ \\
Mobile-mobile annihilation & $\alpha_{2}$ & $4.0[177]$ \\
Dislocation locking & $\alpha_{3}$ & $0.002[177]$ \\
Locked dislocations freed & $\alpha_{4}$ & $0.002[177]$ \\
Cross slip & $\alpha_{5}$ & 0.0 \\
Immobile-mobile annihilation & $\alpha_{6}$ & $0.1[177]$ \\
Initial total mobile dislocation density & $\rho_{o}^{\text {mobile }}$ & $0.5 \times 10^{6} 1 / \mathrm{mm}^{2}[10]$ \\
Initial total immobile dislocation density & $\rho_{o}^{\text {immobile }}$ & $0.5 \times 10^{6} 1 / \mathrm{mm}^{2}[10]$ \\
\hline
\end{tabular}

\subsubsection{Bicrystal in Shear Results}

In this section we present the simulation results from the series of sheared bicrystal simulations. We focus on the variation among the single crystal and two bicrystal 
simulations to determine that the GND model addition to the CDD crystal plasticity framework is sensitive to changes in the grain boundary angle. Because of our interest in the distribution patterns of the solution variables on the bicrystals, we present the results spatially across the bicrystal rather than as averaged stress-strain curves.

To mitigate any impact from the free surfaces, we inspect the results on the midplane of the bicrystal geometry, at $z=0.1 \mathrm{~mm}$ on the undeformed mesh, see Figure 4.8 for reference. These results are sampled at the onset of plasticity when the bicrystal has only been subjected to an effective Lagrangian strain of $2.68 \times 10^{-4}$ $\mathrm{s}^{-1}$. These simulations have run for approximately 1008 hours on 44 processors each. As noted before in Section 4.3.2, the computational overhead associated with the nodal patch recovery algorithm used to calculate the GND density is large. This early collection of the solution results enables us to inspect the distribution patterns before any significant hardening of the crystal has occurred.

At this early point in the deformation, the effective second Piola Kirchhoff stress response of the single crystal and the two bicrystals is nearly identical, Figure 4.9 . The single crystal, Figure 4.9a, demonstrates slightly higher stresses at the corners of the mesh than either the small grain boundary angle bicrystal, Figure 4.9b, or the medium grain boundary angle bicrystal, Figure 4.9c. All three simulations display pockets of lowest stress on the center faces parallel to the $y$ axis. This similarity in the effective stress pattern among all three crystal simulations indicates that the 


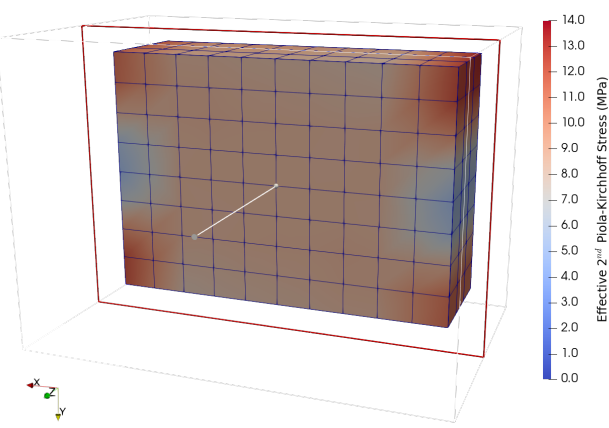

(a) Single crystal

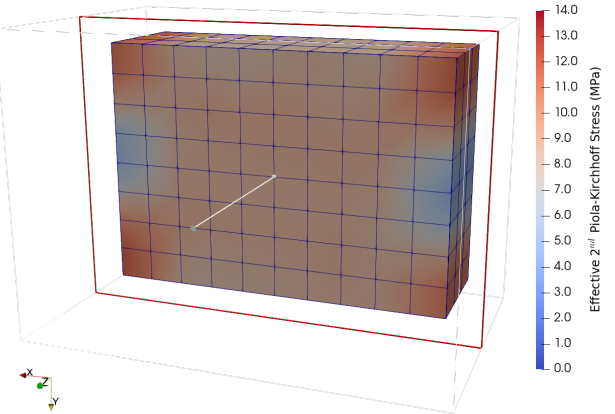

(b) Small boundary angle bicrystal

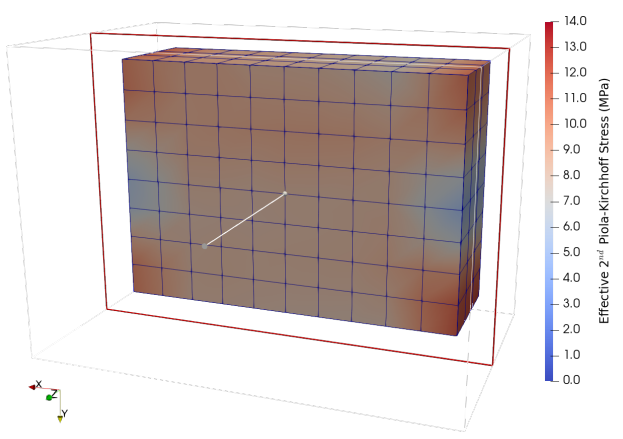

(c) Medium boundary angle bicrystal

Figure 4.9: The effective second Piola-Kirchhoff stress, shown on the midplane of the bicrystal geometry $(z=1.0 \mathrm{~mm})$, demonstrates little change across the center of the bicrystal geometry among the three different misorientation angle simulations, see Table 4.4. These presented results are sampled at the onset of plasticity; thus any variation in the effective stress profiles across the three simulations due to the GND density, Figure 4.10, may not yet be observable. 
solutions are still dominated by an elastic stress response at this point in the simulation. This similar stress response is in line with our expectations for the onset of plasticity.

The influence of the different grain boundaries, listed in Table 4.4, is clearly demonstrated by the distribution of the GND densities. Both the pattern and the intensity of the GND density varies among the three different simulations, shown in Figure 4.10. Note that the midplane indicator line intersects the bicrystal geometry at the grain boundary, which aids in distinguishing the patterns of GND distributions.

In the single crystal simulation, Figure 4.10a, the GND distribution follows a similar pattern as in the beam bending simulations in Section 4.3.2: higher concentrations of GNDs are seen on the edges of the beam. This distribution pattern echos the effective stress pattern, and a minimum of GND density is observed in the center third of the single crystal geometry.

While both bicrystals demonstration concentrations of the GND density on either side of the grain boundary, the response of the two bicrystal simulations differs from the single crystal simulation and from each other. We note that in both bicrystal simulations the concentration of GND density is lowest along the grain boundary region. The concentrations of GNDs within the grains of the two bicrystals clearly demonstrate the influence of the grain boundary on the microstructure evolution within the grain interiors. 


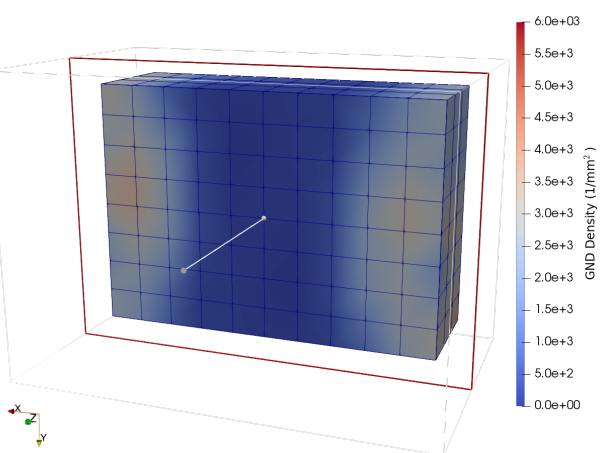

(a) Single crystal

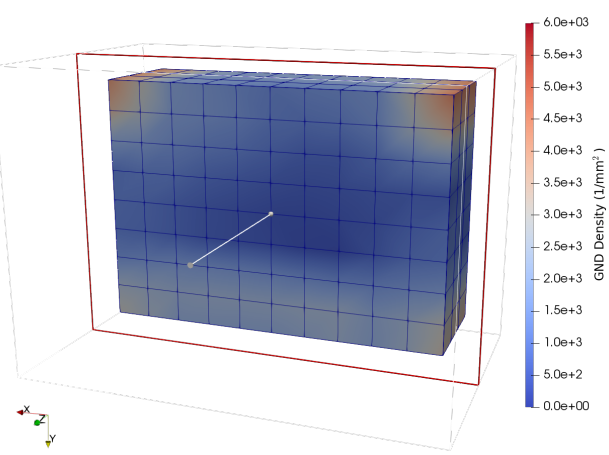

(b) Small boundary angle bicrystal

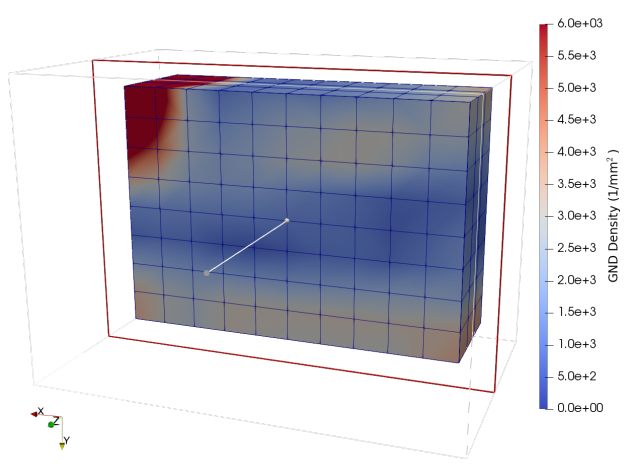

(c) Medium boundary angle bicrystal

Figure 4.10: The variation of the GND density is evident with the increasing grain boundary misorientation angle, even at the onset of plasticity. In the single crystal, Figure 4.10a, the GND density is concentrated at the lateral edges, while in the bicrystals the concentration of GND density is higher in the regions away from the boundary. In the small grain boundary angle simulation, Figure $4.10 \mathrm{~b}$, the GNDs cluster in the corners. The concentration of GND density clusters in both grains is larger in the medium grain boundary angle simulation, Figure 4.10c. These results are from the onset of plasticity. 
In the case of the small angle grain boundary bicrystal, Figure 4.10b, the clusters of higher GND density demonstrate a noticeable difference between the top and bottome grains. While the bottom grain has a near even distribution of GND density across the lower edge, the top grain shows clusters of GND density in the upper corners, away from the grain boundary.

The medium grain boundary angle bicrystal demonstrates an unequal GND density pattern in each of the two grains, Figure 4.10c. While the bottom grain retains a similar GND density pattern and intensity as we observed in the small angle bicrystal, in the top grain the GND density cluster forms a distinct pattern. The upper right corner of the top grain has a significantly larger and more intense concentration of GND density compared to the small angle bicrystal top grain. The medium grain boundary bicrystal also demonstrates a GND density in the center of the top grain. The centered GND cluster has a similar intensity to the bottom grain, also the top grain GND cluster is centered in the middle of the top grain.

The significant variation in the response of the GND density among the single crystal and the two bicrystal simulations indicates that the GND model addition to the CDD crystal plasticity model is sensitive to changes in the grain boundary angle; therefore, this model is a suitable tool for capturing the influence of grain boundaries on the mechanical response of polycrystalline geometries. 


\subsection{Conclusions and Future Recommendations}

In this chapter we have introduced a method for calculating a total density of geometrically necessary dislocations through the application of a nodal ZZ-patch recovery algorithm. We have applied this model to a set of two different benchmark problems to verify the behavior of the GND density model. This nodal patch recovery GND model demonstrates mesh independence of the solution. Furthermore the model shows observable sensitivity to changes in the grain boundaries in polycrystalline simulations. Because of these attributes, the GND model we have presented here is an acceptable addition to the CDD crystal plasticity framework.

Paramount among the potential future work for this GND density model addition is the need to improve the method of collecting the plastic velocity gradient, $L^{P}$, material property for the nodal patch recovery zz-patch algorithm. The additional computational time required by the nodal patch recovery material property reinitialization process is a significant hindrance. The two orders of magnitude increase in compute time for the nodal patch recovery method, as discussed in Section 4.3.2, prevents the current GND model addition from being used in any large strain problems of interest at present. Once these numerical issues are addressed, expanding the range of element point neighbors for the nodal patch recovery method, Section 4.1.2, to second and third levels could aid in expanding the mesh independence of the 
approach.

The GND model addition to the CDD crystal plasticity framework can be applied to problems in which size effects or grain boundary roles are important to the mechanical behavior response. The results of this combined crystal plasticity framework can be used to study the impact of realistic and complex polycrystalline microstructures in a variety of operating enviroments. 


\section{CHAPTER 5. TWINNING VOLUME EVOLUTION}

\section{MODEL ADDITION}

In addition to the $\alpha$ iron used in the reactor pressure vessel, FCC nickel-based super alloys, such as Inconel 690 and Inconel 718, are used in the steam generation components of current nuclear reactor power plants [25]. Nickel-based alloys are also being considered for use as structural materials in GenIV molten salt reactors $[165,199]$. In these applications the nickel alloys are exposed to long term high temperature operating conditions, albeit under lower pressures than in current light water reactor pressure vessels. Thermal aging, therefore, in addition to irradiation damage, must be considered in the study of the microstructure evolution of nickel alloys in nuclear power plant applications.

The use of nickel alloys for piping materials in chemical industry processing plants over decades provides a set of information on the in-operation aging of commercial nickel alloys. Several studies have analyized the microstructure of nickel-based alloys after 50,000 hours to 70,000 hours of in-service operation at temperatures of $450^{\circ} \mathrm{C}$ to $600^{\circ} \mathrm{C}[184,175,164,193,123]$. Among the several precipitates and particles which form in these complex alloys is the formation of Long Ranged Ordered (LRO) $\mathrm{Ni}_{2} \mathrm{Cr}$ precipitates [112]. These coherent precipitates form within nickel alloys after long term exposure to relatively low temperatures [200]. Alloying components such 
as iron and molybdenum will shift the temperature range and aging time required for these LRO precipitates to form $[122,188]$, yet these precipitates persist as a microstructural feature in nickel alloys. These LRO precipitates are also known to increase the hardness of nickel based alloys [112, 201].

The extensive aging time to form the LRO precipitates in commercial nickel based alloys is not conducive to studying the impact of LRO precipitates on microstructures. Many research groups have thus selected model binary alloys, which form the LRO precipitates after reasonable aging times $[113,183,200,172]$. These studies connect the formation of $\mathrm{LRO} \mathrm{Ni}_{2} \mathrm{Cr}$ precipitates with increased hardness of the model alloy. Generally 10,000 hours has been determined to be enough aging time to allow for the complete formation of the LRO precipitates in the binary model alloy [183]. The relative simplicity of the binary $\mathrm{Ni}_{2} \mathrm{Cr}$ model alloy offers the ability to focus on the interaction of the LRO precipitates with mobile dislocations during deformation, using our CDD crystal plasticity framework.

\subsubsection{Micropillar Compression Experiments and Simulations}

Micropillar compression experiments were first introduced by Uchic et al. and Dimiduk et al. as a way to study the effect of smaller sample size on the stress response of a material $[190,38]$. Micropillar experiments have contributed to the un- 
derstanding of how different deformation mechanisms control the material behavior as the sample size changes. Simulations have also been applied to micropillar compression problems, particularly dislocation dynamics and crystal plasticity simulation models. Dislocation dynamics simulations of micropillar compression tests track individual dislocation lines and thus can identify different types of dislocation and source behavior with varying micropillar diameter and dislocation densities [3, 127, 83, 43].

For these advantages, dislocation dynamics simulations are limited to smaller geometries, often modeling only the micropillar itself, because of the computational load of tracking each individual dislocation. Additionally, dislocation dynamics simulations often apply larger strain rates than are applied in micropillar compression experiments [70]. Both of these limitations can be mitigated with crystal plasticity models, although the ability to track individual dislocations is lost in the continuum level approach. Since crystal plasticity models track continuum level dislocation densities, as we've discussed in Section 2.3.2, the appropriate model additions to capture the size-effect observed in micropillars is an area of current research. In particular, for small diameter micropillars, the forest-type hardening model used to capture dislocation interactions in bulk materials is not sufficient to capture the increase in strength of micropillars [38]. Several crystal plasticity studies have suggested the use of strain gradient terms, including the geometrically necessary dislocations we discussed in Chapter 4 [92, 206, 105], although the applicability of these terms to micropillars 
remains a subject of some disagreement in the field $[38,70]$.

Setting aside the disagreements about the role of geometrically necessary dislocations, simulation studies of micropillars agree on the importance of boundary conditions to correctly model the micropillar. Akarapu et al. and Kuroda both demonstrate a significant variation in the simulation results with varied applications of the loading boundary conditions on top of the micropillar [3, 92]. The approaches used for the boundary conditions at the bottom of the micropillar vary significantly. In dislocation dynamics simulations, which model only the micropillar itself, fixed displacement boundary conditions are applied to the pillar base while dislocations are allowed to exit the surface [3]. Crystal plasticity studies which employ the same micropillar-only geometry and fixed boundary conditions on the pillar base [70, 38, 38] artificially prevent the continuum level representation of the micropillar from interaction with the substrate.

Recent studies have demonstrated the importance of including the substrate geometry on the stress-strain curve predicted by the simulation for an isotropic plastic material [47] Other crystal plasticity simulations of micropillar compression tests have included the substrate within the meshed problem geometry, despite the additional computational load introduced $[155,74]$. These crystal plasticity studies have addressed the computational load associated with including the substrate geometry by reducing the number of elements in the mesh through mesh coarsening. The aggres- 
sive mesh coarsening employed in these studies could, however, fail to fully capture the interactions of the substrate and the micropillar.

Beyond the study of size-dependence of material strength, micropillar compression tests are useful for determining material properties such as yield stress and work hardening behavior [38]. In cases where limited amounts of material are available, as is the case with the 10,000 hours thermally aged $\mathrm{Ni}_{2} \mathrm{Cr}$ alloy, micropillar compression tests offer the ability to determine the stress-strain behavior in a more direct approach than through nanoindentation, another common experimental technique. Furthermore Cruzado et al. argue that for a nickel-based alloy with a deformation behavior governed by dislocation-particle interactions, micropillar compression tests can be used to directly calibrate a crystal plasticity model [34]. Because of the limited amount of the 10,000 hours aged binary alloy, micropillar compression testing was selected as the method to mechanically test single crystals of both aged and unaged $\mathrm{Ni}_{2} \mathrm{Cr}$ alloy. Crystal plasticity is a useful simulation tool in this situation to extend information from experimental data sets limited by available material to polycrystalline structures as we have shown in Section 3.4. We have therefore focused on replicating the micropillar compression tests with our CDD crystal plasticity model as a first step towards the prediction of engineering scale mechanical behavior based on the evolution of the thermally aged microstructure of nickel based alloys.

In this chapter we present first the addition of a twinning model to the CDD 
crystal plasticity framework, with coupling between the glide and twin dislocations. We benchmark our results from the twinning model implementation with a set of verification tests with compression and tensile loads in orientations know to promote and to mitigate twinning growth. In the second part of this chapter we present preliminary results of the micropillar compression simulations to demonstrate the impact of the twinning model addition to the CDD crystal plasticity framework.

\subsection{Twinning Model Implementation}

Experimental data on a set of [111] and [100] oriented micropillars was collected on both unaged and 10,000 hours aged single crystals of $\mathrm{Ni}_{2} \mathrm{Cr}$ alloy $[189,182]$. During the experimental testing of these micropillars, twinning was observed in the [100] orientation micropillars $[181,188]$. Thus, in order to model the active physical deformation mechanisms active in the $\mathrm{Ni}_{2} \mathrm{Cr}$ alloy, we must add a deformation twinning model to our CDD crystal plasticity framework. We justify the use of the simplistic twinning model [76] over other twinning models developed for nickel-based alloys $[77,169]$ with the small size of the LRO precipitates. Because the average diameter of the LRO precipitates is under $20 \mathrm{~nm}$ these precipitates cannot hold both the tail and lead partials of a dislocation in order to nucleate a twin within the precipitate [89]. Given this physical limitation on the twinning mechanisms active in our binary 
model alloy of interest, $\mathrm{Ni}_{2} \mathrm{Cr}$, we have focused our work in this chapter on the crystal plasticity framework development to enable two different contributions to the plastic velocity gradient.

Following [198] we incorporate the deformation twinning into the plastic velocity gradient as a pseudo-slip mechanism. We implement a variation of the twinning model introduced by Kalidindi with an additive decomposition of the plastic velocity gradient.

$$
L^{P}=\left(1-f_{t}\right) \sum_{\alpha} \dot{\gamma}^{\alpha} s_{o}^{\alpha} \otimes m_{o}^{\alpha}+\sum_{\beta} \dot{\gamma}^{\beta} s_{o}^{\beta} \otimes m_{o}^{\beta}
$$

where $f_{t}$ is the total volume fractions of the twins on all of the twin systems, and the superscript $\alpha$ indicates the glide slip systems and the superscript $\beta$ indicates the twinning system. As we discussed in Section 2.2.3, the glide slip systems for an FCC material are given in Table 2.2 and the twin systems are listed in Table 2.5. Implicit in Eq (5.1) is the assumption that the twinned portions of the crystal do not undergo additional dislocation glide, as assumed in [163]. We note that the direction contribution of the twin shear into the plastic velocity gradient will allow both glide and twin dislocations to influence the calculation of the geometrically necessary dislocations discussed in Chapter 4 .

The twin slip increment model we have implemented is the power law type model first proposed by Kalidindi [75]. In incremental form the shear slip due to twinning 
is given as

$$
\Delta \gamma^{\beta}=\dot{\gamma}_{o}\left(\frac{\tau^{\beta}}{g_{\text {twin }}^{\beta}}\right)^{1 / m} \Delta t \text { if } \tau^{\beta}>0 \text { and } f_{T}<f_{\text {limit }}
$$

where we use the same shear reference rate $\dot{\gamma}_{o}$ and power law exponent $m$ as in the glide dislocation velocity model, Eq (2.65) as suggested by [76]. The resistance of the system to twinning, $g_{\text {twin }}^{\beta}$ is given by Eq (5.4). We introduce coupling to the CDD glide dislocations through the twinning system resistance. The total twin volume fraction, $f_{T}$, used in the calculation of the plastic velocity gradient in $\mathrm{Eq}$ (5.1), is calculated directly from the twin slip increment.

$$
f_{T}=\sum\left(\frac{\Delta \gamma^{\beta}}{\gamma_{t w}}+f_{\text {old }}^{\beta}\right)
$$

where $\gamma_{t w}$ is the characteristic twin shear [76] and $f_{\text {old }}^{\beta}$ is the volume fraction of twins on $\beta$ twin system from the previous timestep. For an FCC material, which we consider here, the characteristic twin shear is $1 / \sqrt{2}[5]$. Although the power law model, Eq (5.2), has been described as phenomenological, we show in Chapter 6, for the case of glide dislocations, how the power law model can be connected to physically based dislocations through the Orowan relation.

We note that Kalidindi's calculation of the Cauchy stress includes a rotation of the elasticity tensor for the twinned volume fraction [76]. In our calculations of the second Piola Kirchhoff stress and the related Cauchy stress we have not included this rotation of the elasticity tensor for the twinned volume in our current implementation. We justify this current simplification with the small volume of twins both predicted in 
our simulation and observed in the experimental data [181, 188]. Future development of this constitutive twinning model for applications with larger twinning volumes from more twin dislocations, including NiCr alloys such as Alloy 690 and Alloy 625, will include the rotation of the elasticity tensor for the calculation of the stress.

\subsubsection{Coupling to CDD Glide Dislocation Evolution}

Interactions between glide dislocations and twins increase the resistance of the crystal to the motion and growth of both dislocation types. In light of this observation we have introduced coupling of the twin dislocation and glide dislocation evolution through the resistance components of the two evolution models.

We treat the resistance of twin slip as an additive decomposition

$$
g_{\text {twin }}^{\beta}=g_{\text {tw-o }}+g_{\text {forest }}+g_{\text {forest-twin }}
$$

where $g_{t w-o}$ represents the lattice friction contribution to the twin resistance, $g_{\text {forest }}$ is the forest hardening from glide dislocations, $\mathrm{Eq}(2.71)$, and $g_{t w-f o r e s t}$ is a forest hardening type term to account for the accumulation of twins. The lattice friction twin system hardening has the simple form of

$$
g_{t w-o}=\tau_{p s}
$$

where, following the summary of [161] $\tau_{p s}$ is the Peierls stress. The third term in Eq (5.4) represents the hardening due to twins. Twins are known to increase the 
system hardening for noncoplanar systems over other coplanar systems [161]. Thus several crystal plasticity models introduce twinning resistance models with different treatments for coplanar and noncoplanar systems [76]. In our model we have adopted a simpler approach in which we treat the contributions from all twin systems equally,

$$
g_{\text {forest-twin }}=\alpha_{t f} \mu \sqrt{f_{T}}
$$

which is similar to the approach used to calculate the forest hardening from glide dislocations, Eq (2.71). We acknowledge that this form of slip resistance on the twin systems calculates an equal value of resistance for all twin systems, both active and inactive.

The influence of the twins on the evolution of the glide dislocation is incorporated into the calculation of the mean free glide path, Eq (2.69), rather than directly into the glide resistance. We modify the mean free glide path with a correction factor that is a function of the total twin volume.

$$
l_{\text {inv }}^{(\alpha)}=\beta_{\text {path }} \sqrt{\frac{\sum_{\beta} \omega^{(\alpha \beta)}\left(\rho_{\text {mobile }}^{(\beta)}+\rho_{\text {immobile }}^{(\beta)}\right)}{1-f_{T}}}
$$

where the denominator is the twin volume correction factor [63]. As in Chapter 4 with geometrically necessary dislocations, we account for the additional obstacles to glide dislocation only in the mean free glide path to avoid artificially overhardening the glide slip systems.

The combination of the glide forest hardening term in the twin resistance ex- 
pression, Eq (5.4), allows us to complete the two-way coupling of these two different deformation mechanisms.

\subsubsection{Addition of LRO Precipitate Contribution to Glide Resistance}

As small coherent particles within the disordered $\mathrm{Ni}_{2} \mathrm{Cr}$ alloy matrix, the small LRO precipitates act as barriers to glide dislocation motion. We modify the glide dislocation resistance, $\mathrm{Eq}$ (2.70) with an additional term for these precipitates

$$
g^{(\alpha)}=g_{o}^{(\alpha)}+g_{\text {forest }}^{(\alpha)}+g_{\text {apb }}^{(\alpha)}
$$

where $g_{a p b}^{(\alpha)}$ is the weakly coupled anti-phase boundary shearing defined in Eq (2.74). Given the small size and coherent nature of the LRO precipitates, we have adopted the anti-phase boundary shearing model for tertiary $\gamma^{\prime}$ particles, [89], to capture the LRO precipitate interaction with mobile dislocations. This model is only used for the simulations of fully formed LRO precipitates after 10,000 hours of thermal aging.

\subsection{Single Crystal CDD with Twinning Simulations}

Experimental observations of twin formation and growth in FCC materials have established a conventional wisdom for those loading orientations which demonstrate twinning. FCC materials are known to twin in the [100] orientation when loaded in 
compression but not in tension; conversely, tensile loading in the [111] orientation demonstrates twinning while compression does not [110]. This expected behavior is a consequence of the unidirectional nature of twins, which we have captured in Eq (5.2). While the same twin systems receive the largest absolute shear stress in the [100] orientation, only in compression are these shear stresses positive and thus capable of promoting twin growth.

We use this set of four loading directions and orientations, [100] in compression, [100] in tension, [111] in compression, and [111] in tension, to verify our general implementation of the twin model addition to the CDD crystal plasticity framework. The single crystal simulations were performed on a $1 \mathrm{~mm}^{3}$ cube mesh consisting of 216 Hex8 elements. Symmetric boundary conditions were used and a displacement loading rate corresponding to a strain rate of $\pm 1.0 \times 10^{-3} \mathrm{~s}^{-1}$ was applied, depending on the specified loading direction. The elastic constants and material parameters used in this set of simulations are given in Table 5.1 and the glide dislocation evolution parameters are given in Table 5.2. 
Table 5.1: The elasticity tensor and hardening material parameter values used in the CDD crystal plasticity for twinning model addition simulations of single crystal $\mathrm{Ni}_{2} \mathrm{Cr}$.

\begin{tabular}{ccl}
\hline Parameter & Value & Description \\
\hline$C_{11}$ & $332.4 \times 10^{3} \mathrm{MPa}$ & Elastic constant, [20] \\
$C_{12}$ & $186.8 \times 10^{3} \mathrm{MPa}$ & Elastic constant, [20] \\
$C_{44}$ & $72.8 \times 10^{3} \mathrm{MPa}$ & Elastic constant, [20] \\
$\mu$ & $72.8 \times 10^{3} \mathrm{MPa}$ & Shear modulus, [20] \\
$b$ & $2.52 \times 10^{-7} \mathrm{~mm}$ & Burgers vector, Eq (2.59) \\
$\alpha_{m b h}$ & 0.4 & Forest hardening coefficient, Eq (2.71) \\
$\Omega^{\alpha \alpha}, \Omega^{\alpha \beta}$ & 1.0 & Self- and latent-hardening coefficient, Eq (2.71) \\
$\omega^{\alpha \alpha}, \omega^{\alpha \beta}$ & 1.0 & Mean free glide path, Eq (2.69) \\
$\tau_{p s}$ & $3.64 \mathrm{MPa}$ & Estimated isotropic Peierls strength, [176], Eq (3.2) \\
$T$ & $298 \mathrm{~K}$ & Temperature, Eq (3.3) \\
\hline
\end{tabular}


Table 5.2: Values of the dislocation evolution parameters used in the simulations for the single crystal twinning model verification benchmark simulations. These parameters are for the CDD dislocation evolution model, $\mathrm{Eq}$ (2.67) and Eq (2.68) and are fit for FCC aluminum [177], and for the twinning evolution model.

\begin{tabular}{lcl}
\hline Dislocation Evolution Interaction & Constant & Value \\
\hline Frank-Read generation & $\alpha_{1}$ & $0.04[177]$ \\
Mobile-mobile annihilation & $\alpha_{2}$ & $4.0[177]$ \\
Dislocation locking & $\alpha_{3}$ & $0.002[177]$ \\
Locked dislocations freed & $\alpha_{4}$ & $0.002[177]$ \\
Cross slip & $\alpha_{5}$ & 0.001 \\
Immobile-mobile annihilation & $\alpha_{6}$ & $0.1[177]$ \\
Annihilation capture radius & $R_{c}$ & $15 b \mathrm{~mm}, \mathrm{Table} 2.4$ \\
Initial mobile dislocation density & $\rho_{o}^{\text {mobile }}$ & $0.5 \times 10^{6} 1 / \mathrm{mm}^{2}[10]$ \\
Initial immobile dislocation density & $\rho_{o}^{i m m o b i l e}$ & $0.5 \times 10^{6} 1 / \mathrm{mm}^{2}[10]$ \\
Characteristic twin shear & $\gamma_{t w}$ & $0.707,[161]$ \\
Twin forest hardening coefficient & $\alpha_{t f}$ & $1.0 \times 10^{3}, \mathrm{Eq}^{2}(5.4)$ \\
\hline
\end{tabular}




\subsubsection{Twinning Verification Set Results}

The results of our benchmark simulations, Figure 5.1, demonstrate the CDD twinning model produces results in line with the conventional expectations. Compression loading in the [100] orientation and tensile loading along the [111] orientation both demonstrate twin volume growth.

The [111] orientation compression loading case demonstrates a minimal amount of twin volume growth while the tensile [100] loading shows no twin volume growth, as expected. We note that in both cases with twin volume fraction growth, Figures 5.1a and 5.1d, the effective stress response is around $10 \mathrm{MPa}$ higher than for the cases without twin growth, Figures 5.1b and 5.1c. This increased stress effect is more pronounced in the [111] orientation where fewer glide dislocation systems are active. The change of the effective stress response indicates that the twin slip increment implementation has been included in the calculation of the stress through the plastic velocity gradient modification, Eq 5.1.

We note that the volume of twin dislocations, even in the twin promoting loading directions and orientations, is less than $1 \%$. This low value of twin volume in the matrix justifies our simplifying assumption to neglect the rotation of the elasticity tensor for the twinned volume of the crystal lattice. 


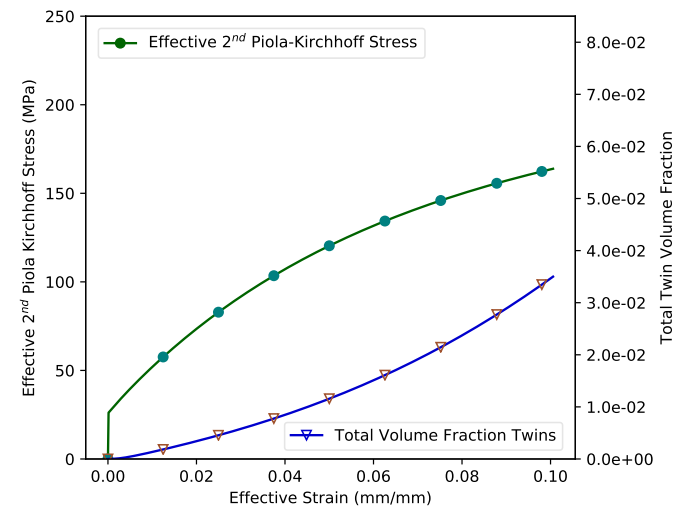

(a) Compression in the [100] direction

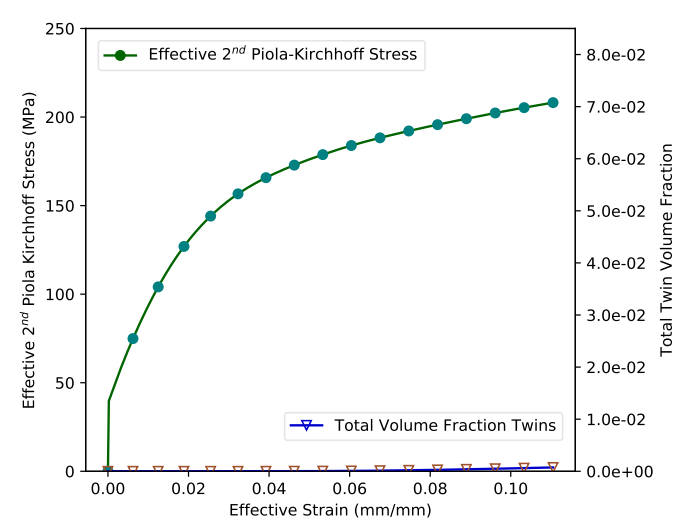

(c) Compression in the [111] direction

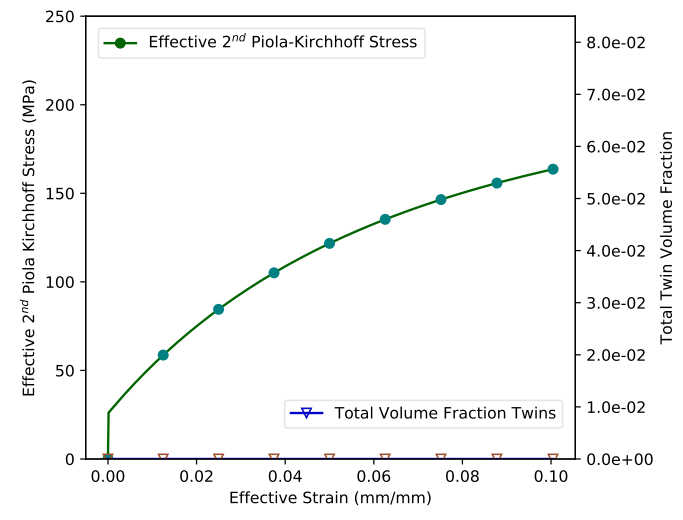

(b) Tension in the [100] direction

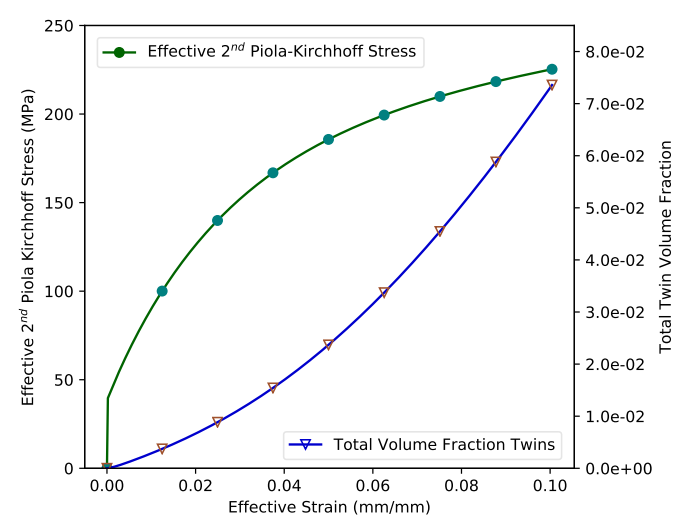

(d) Tension in the [111] direction

Figure 5.1: The twinning model addition to the CDD crystal plasticity framework demonstrates the formation of twins in the loading directions known to form twins in FCC materials: compression loading in [100] and tensile loading in [111]. As observed in experimental tests, tensile loading in the [100] direction and compression loading in the [111] direction do not form twins. These results indicate the proper implementation of the twinning model. 


\subsubsection{Mesh Convergence Study}

In conjunction with the verification of the twinning model, we performed a mesh convergence study on each of the four loading and orientation sets The parameters given in Tables 3.1 and 3.2 were used in these mesh convergence studies, and the same symmetry and tensile loading boundary conditions as used in Section 5.2.

A series of meshes, in which the number of element in each linear direction was successively increased, were used to run the same crystal plasticity simulation: $8\left(2^{3}\right)$, $64\left(4^{3}\right), 216\left(6^{3}\right)$, and $512\left(8^{3}\right)$ total elements in the $1 \mathrm{~mm}^{3}$ cube. The results of this mesh convergence study are shown in Figure 5.2 for the [100] orientation, and the results of the mesh convergence study in the [111] orientation are given in Figure 5.3.

Among the four mesh convergence comparisons in Figure 3.2, the stress strain response appears reasonably converged by $216\left(6^{3}\right)$ elements. Therefore, in the simulation of the micropillar compression tests, Section 5.3, we have taken the reference mesh size as 6 linear elements per dimension as the minimum. 


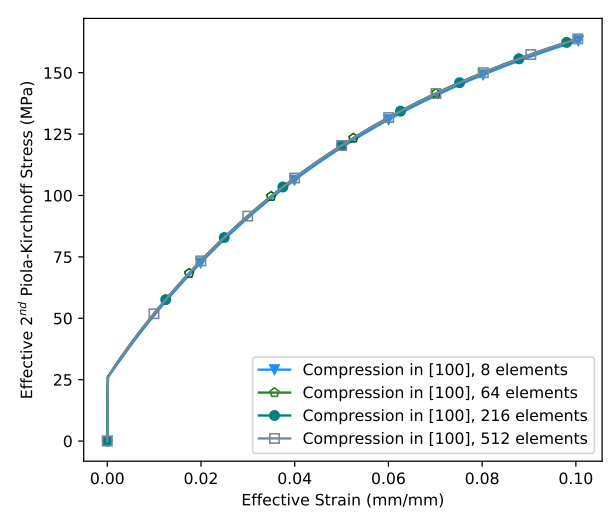

(a) Effective $2^{\text {nd }}$ Piola Kirchhoff stress response to compression in the [100] direction

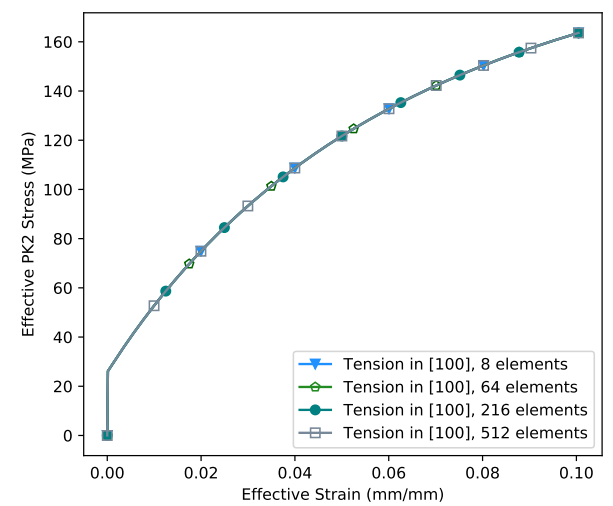

(c) Effective $2^{\text {nd }}$ Piola Kirchhoff stress re-

sponse to tension in the [100] direction

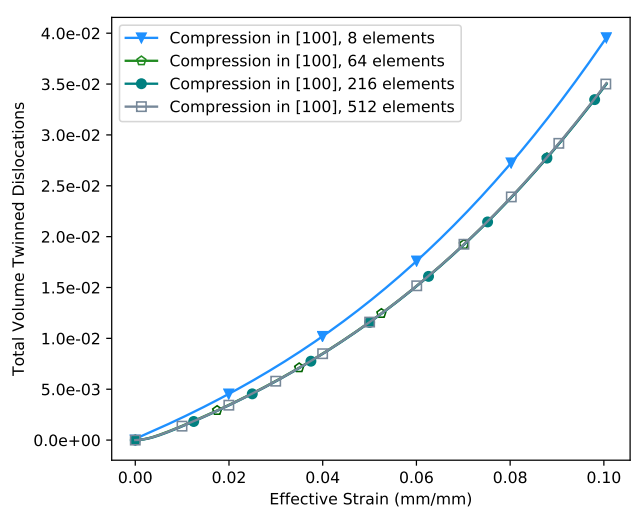

(b) Total volume of twins under compression along the [100] direction

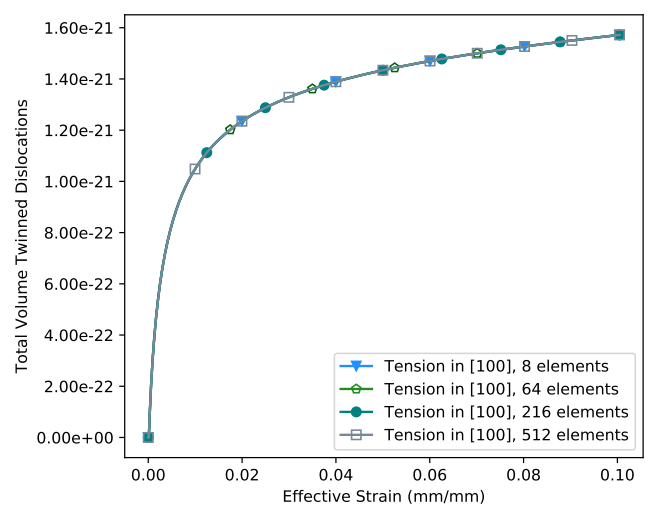

(d) Total volume of twins under tensile loading in the [100] direction

Figure 5.2: The stress response of the CDD crystal plasticity simulations with the twinning model addition does not demonstrate mesh dependence under compression, Figure 5.2a, nor in tension, Figure 5.2c. Only under compression does the total twin volume demonstrate mesh sensitivity on the coarsest mesh, Figure 5.2b. Note that the scales used for Figures $5.2 \mathrm{~b}$ and $5.2 \mathrm{~d}$ are not equivalent. 


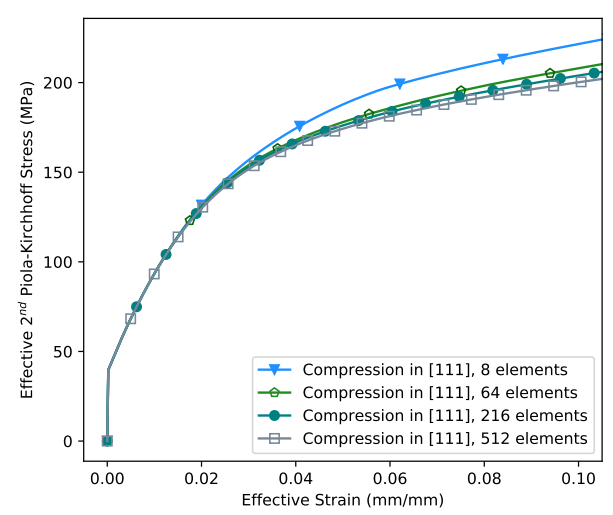

(a) Effective $2^{\text {nd }}$ Piola Kirchhoff stress response to compression in the [111] direction

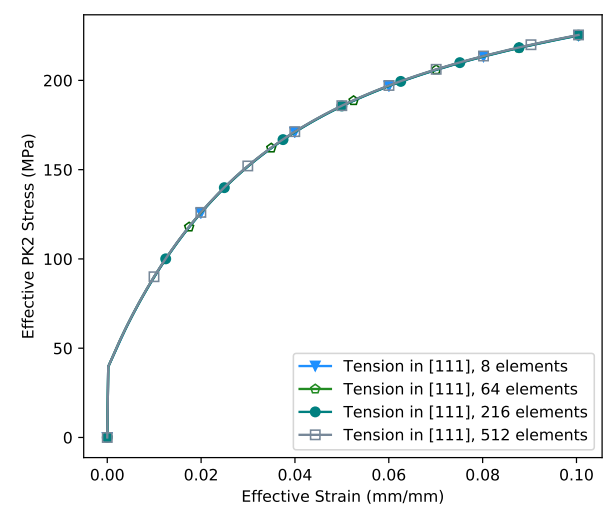

(c) Effective $2^{\text {nd }}$ Piola Kirchhoff stress response to tension in the [111] direction

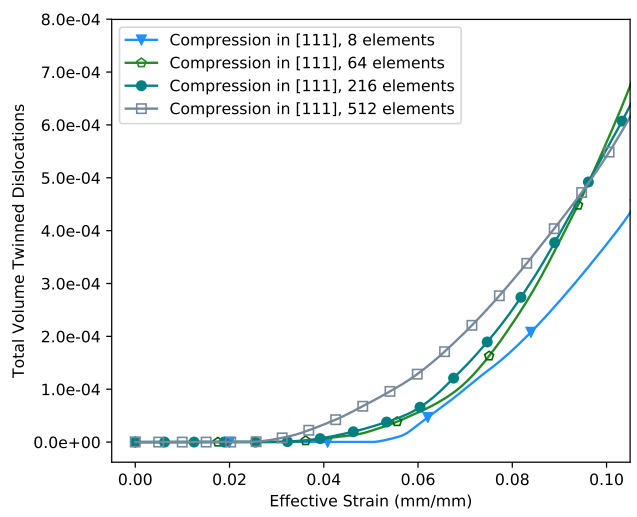

(b) Total volume of twins under compression loading in the [111] direction

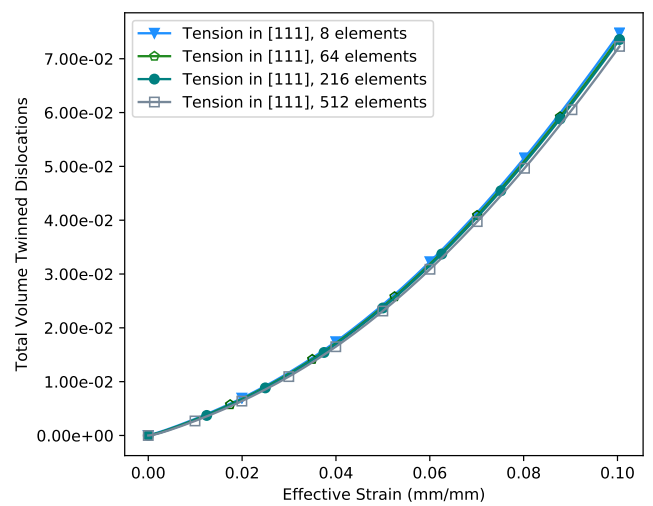

(d) Total volume of twins under tensile loading in the [111] direction

Figure 5.3: As in the [100] loading direction, the response of the crystal plasticity simulations with the twinning model addition demonstrate little mesh sensitivity under tensile loading which promotes twinning growth, Figure 5.3c and 5.3d. Under compression the stress response, Figure 5.3a, and the twin volume, Figure 5.3b, demonstrate mesh sensitivity, particularly on the coarser two meshes. 


\subsection{Micropillar Compression Nickel-Chromium Simulations}

With the successful benchmark simulations of our twin slip implementation, we turn to the simulation of $\mathrm{Ni}_{2} \mathrm{Cr}$ alloy micropillars, both aged and unaged. To align with the experimental procedure described in [189] we simulate a set of [111] and [100] micropillars, with aged and unaged $\mathrm{Ni}_{2} \mathrm{Cr}$. We adopt a nominal micropillar height of $4 \mu \mathrm{m}$ with a nominal width and depth of $2 \mu \mathrm{m}$, as shown in Figure 5.4. In our simulations we model both the micropillar and the substrate with the CDD crystal plasticity framework, including the twinning model addition, with a uniform mesh element size. We mitigate the computation load with a check on the dislocation velocity value, and only compute the dislocation density evolution and associated slip increment, shown in green in Figure 2.5 when a dislocation velocity is nonzero in an element.

Additionally, our use of the cube shape for the substrate component, shown in Figure 5.4, enables the application of boundary conditions which better mimic the position of the modeled substrate portion within the larger substrate plane, compared to the cylinderical shapes adopted by $[155,74]$. We have applied zero displacement boundary conditions normal to all of the substrate surfaces except the surface with the micropillar. These zero displacement boundary conditions capture the inability of the modeled substrate component to move. The displacement rate boundary condition 
of $-0.01 \mu \mathrm{m} / \mathrm{s}$ was applied to the top of the micropillar, as shown in Figure 5.4.

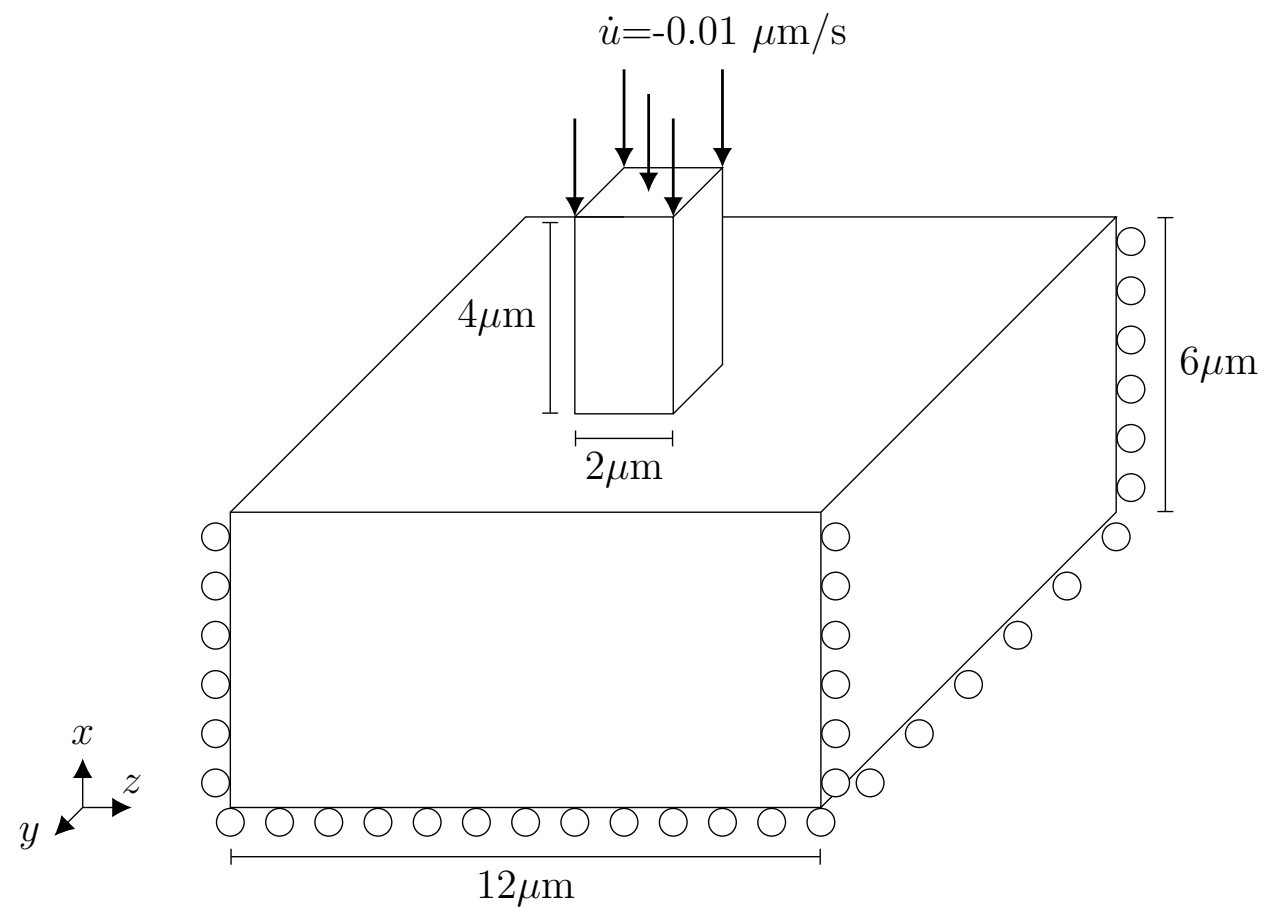

Figure 5.4: The micropillar compression test geometry used to run simulations of the micropillar compression tests on the binary $\mathrm{Ni}_{2} \mathrm{Cr}$ model alloy. The micropillar width and depth have the same dimension, and the substrate width and depth are the same length. The bottom of the micropillar substrate is fixed in the $x$ direction, the front and back sides of the substrate cube are fixed in the $y$ direction as are the right and left sides fixed in the $z$ direction, and a compressive displacement is applied to the top of the micropillar.

Although we relied on elastic constants for a specific nickel-based alloy, 690, for our twinning verification problems, we have elected to apply $\mathrm{Ni}_{2} \mathrm{Cr}$ alloy-specific elastic constants in the micropillar compression simulations calculated rather than measured 
from experimental work. To capture the behavior of the micropillar, including the effect of anisotropy in the elasticity tensor is important; therefore, the isotropic elastic constants given for Alloy 690 [20] are less suitable. Following the trends set by other crystal plasticity models of nickel-based alloys [79, 74, 81], we have adopted a set of aniostropic elasticity constants. Notably these crystal plasticity studies have used an internal lattice friction hardening value, the Peierls stress, several orders of magnitude higher than those estimated by molecular dynamics studies for FCC materials [140, 176]. This higher Peierls stress value will directly impact the stress response predicted by our crystal plasticity simulation by significantly increasing the applied shear stress required to initiate dislocation movement. In our simulations we employ a set of anisotropic elastic constants calculated from first principles specifically for $\mathrm{Ni}_{2} \mathrm{Cr}$ [30]. This study gives separate values of the Peierls stress for edge dislocations and screw dislocations [30]; since we do not distinguish between edge and screw dislocations in our crystal plasticity framework, we use the average of these two Peierls stress values. The list of elastic properties used in our micropillar compression simulations is given in Table 5.3. The LRO precipitate characteristics for the aged micropillar simulations are also listed in this table.

Following the assumption of a low initial dislocation density for the micropillars, similar to that of a well-annealed crystal, we apply a total initial dislocation density of $1.0 \times 10^{6} \mathrm{~mm}^{-2}[100]$ and split this total initial dislocation density evenly among the 
Table 5.3: The elasticity tensor and hardening material parameter values used in the CDD crystal plasticity for the micropillar $\mathrm{Ni}_{2} \mathrm{Cr}$ simulations. The characteristics of the LRO precipitates for the aged micropillar simulations are also listed.

\begin{tabular}{|c|c|c|}
\hline Parameter & Value & Description \\
\hline$C_{11}$ & $230.7 \times 10^{3} \mathrm{MPa}$ & Elastic constant, [30] \\
\hline$C_{12}$ & $78.8 \times 10^{3} \mathrm{MPa}$ & Elastic constant, [30] \\
\hline$C_{44}$ & $71.7 \times 10^{3} \mathrm{MPa}$ & Elastic constant, [30] \\
\hline$\mu$ & $74.5 \times 10^{3} \mathrm{MPa}$ & Shear modulus, [30] \\
\hline$b$ & $2.16 \times 10^{-7} \mathrm{~mm}$ & Burgers vector, [30], Eq (2.59) \\
\hline$\alpha_{m b h}$ & 0.4 & Forest hardening coefficient, Eq (2.71) \\
\hline$\Omega^{\alpha \alpha}, \Omega^{\alpha \beta}$ & 1.0 & Self- and latent-hardening coefficient, Eq (2.71) \\
\hline$\omega^{\alpha \alpha}, \omega^{\alpha \beta}$ & 1.0 & Mean free glide path, Eq (2.69) \\
\hline$\tau_{p s}$ & $191.5 \mathrm{MPa}$ & Estimated Peierls strength, [30], Eq (3.2) \\
\hline$T$ & $298 \mathrm{~K}$ & Temperature, Eq (3.3) \\
\hline$d_{L R O}$ & $2.5 \times 10^{-6} \mathrm{~mm}$ & Average LRO precipitate diameter[61] \\
\hline$f_{L R O}$ & 0.033 & Volume fraction of LRO precipitates [61] \\
\hline$\gamma_{a p b}$ & $2.0 \times 10^{-4}$ & Energy of the antiphase boundary, Eq (2.74) \\
\hline
\end{tabular}

mobile and immobile dislocation densities. The dislocation evolution parameters and initial conditions are the same as those used in the twinning verification study and 
are given in Table 5.2.

In this work we have completed two sets of micropillar compression simulations: an unaged binary $\mathrm{Ni}_{2} \mathrm{Cr}$ alloy set and the same binary alloy after 10,000 hours of thermal aging at $475^{\circ} \mathrm{C}$. We simulate each alloy set in both the [100] orientation and in the [111] orientation. To capture the impact of the thermal aging, we introduce a population of uniformly distributed LRO precipitates. The 10,000 hours of thermal aging were selected to ensure the formation of a stable LRO precipitation population [183]. Because of the difficulties with measuring the diameter of the coherent LRO precipitates [181], many of these studies rely on measurements of lattice contraction to determine if LRO precipitates have formed [172]. Thus only a few studies provide experimental measurements of LRO precipitate diameter, and the potential for experimental error is high [181]. For this reason we have relied on the results of a molecular dynamics study, [61] to provide the average LRO precipitate diameter and volume fraction for our crystal plasticity simulations as listed in Table 5.3.

\subsubsection{Micropillar Compression Simulation Results}

Calculating the stress-strain curve from micropillar compression experiments is not straightforward: the contribution of the substrate to the overall response must be separated from the response of the micropillar alone. The Sneddon correction is 
often applied to extract the stress-strain response [47, 34]. In our study we avoid the need for a correction factor by directly comparing the force-displacement experimental measurement to the simulation results.

In Figure 5.5 we present the results of our CDD simulations with preliminary experimental micropillar compression data [182]. Because these experimental micropillar data are preliminary, we do not expect to see exact alignment in the elastic or plastic regions; instead we use these experimental data to qualitatively access the results of our crystal plasticity simulations. For the unaged micropillar we compare the CDD crystal plasticity simulation results against a force-displacement data collected from a [213] orientated micropillar, Figure 5.5a, and for the thermally aged simulations experimental measurements in the corresponding loading orientations are available, Figure 5.5b.

We note that bulk single crystal experiments indicate that the stress response for loading in the [100] and [111] directions should be higher than the stress response from the [123] loading direction $[178,67,10]$. The higher stress response is a consequence of the multiple slip systems which are active in the [100] and [111] loading directions compared to the only two active slip systems in the [123] type loading orientation. Since the stress response is calculated from the force response, we expect the same trends to hold as we compare the force-displacement curves. With this trend in mind, we recognize that similar plastic regime force response between the 
[100] simulation result and the [213] experimental data indicates an underprediction of the micropillar response by our crystal plasticity framework. Similarly, although the simulation results for the [111] oriented micropillar exceed the experimental data, previous experimental measurements in different loading orientations have determined that the stress response for loading in the [111] direction significantly exceeds the [123] response for FCC materials $[178,67]$. The underprediction of the micropillar force response by our crystal plasticity simulations is apparent in the thermally aged comparison, Figure 5.5b.

Furthermore we note that the simulations predict a minimal difference in the plastic regime force response of the thermally aged simulations compared to the unaged simulations for each loading orientation. In contrast to these predictions, experimental hardness measurements of the unaged and thermally aged binary alloy have recorded a noticeable increase in hardening with the increased presence of LRO precipitate [183]. In fact, hardness tests are one of the most common experimental methods used to determine the presence of LRO precipitates in nickel-based alloys [200, 201, 122]. This slight predicted increase in hardening for the thermally aged materials demonstrates that our crystal plasticity simulation has not fully captured the interaction of the LRO precipitates with the dislocations. 


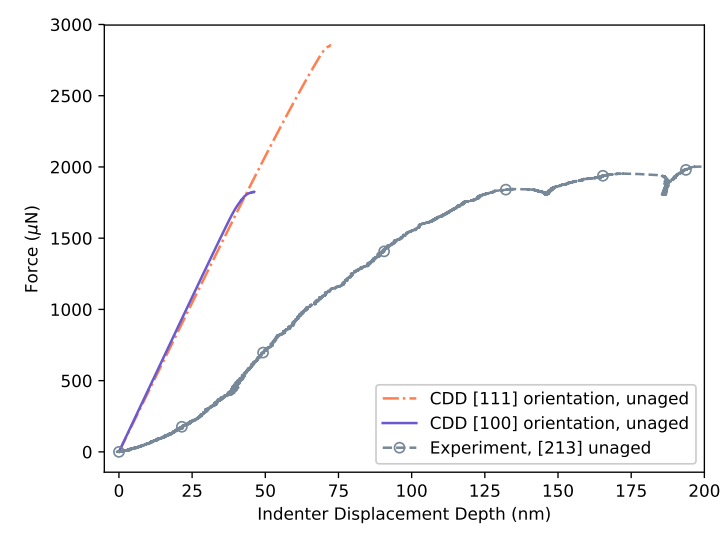

(a) The force response of the unaged [100] and [111] orientated micropillars with the force measured from a [213] orientated micropillar compression experiment [182] provided for reference

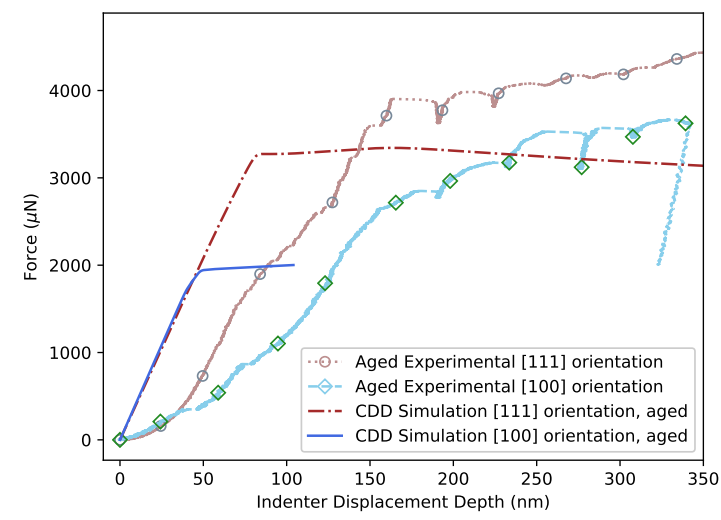

(b) The force response of the 10,000 hours thermally aged [100] and [111] orientated micropillars compared to the force measured from the experimentally compressed micropillars with the same orientation [182]

Figure 5.5: The significant differences in the force measured during the experimental micropillar compression tests and the force predicted by the crystal plasticity simulations indicates that not all of the relevant physics active in the micropillar are captured by our bulk material crystal plasticity model. We suggest further developments to the model or experimental testing of larger diameter micropillars to bring the measured and simulation results into alignment.

The underprediction of the force response by the simulations clearly indicates that our crystal plasticity framework does not capture all of the physics relevant to this problem. Experimental studies of pure nickel micropillars found that a minimum 
micropillar diameter of $20 \mu \mathrm{m}$ was required to replicate bulk material properties [38]. The CDD crystal plasticity models we have employed in these simulation are suitable for bulk materials. Since the micropillar geometry we are attempting to replicate here is an order of magnitude smaller than this threshold, it is reasonable that size effects, which we have discussed previously, may play a role in the simulation underpredictions.

Beyond the difference likely due to size effects, the simulations of the thermally aged micropillar also underpredict the hardening observed in the micropillar experiment force response, Figure 5.5b. Furthermore we note that the thermally aged micropillar force response demonstrates a larger hardening slope than does the unaged micropillar force measurement. Allowing for the different loading orientations, this difference in hardening behavior raises the possibility that the thermally aged LRO precipitates contribute to the work hardening. To capture this work hardening contribution of the LRO precipitates, the interactions between theses precipitates and dislocations should be investigated further. Among the potential interaction mechanisms to consider are bowing of dislocations around precipitates or the pinning of dislocation segments by a pair of precipitates to create a new glide dislocation source, such as the Frank-Read source, Section 2.2.3. Additionally our proposed twinning resistance model, Eq (5.4), does not include a contribution from the LRO precipitates.

The apparent softening in the force response of the thermally aged micropillar 
simulations is the result of improper boundary conditions, which have allowed the top micropillar surface to slide out. To address this additional movement of the top surface, we will explore the use of frictional and zero displacement boundary conditions for the displacements tangential to the top micropillar surface. We expect that these boundary conditions will prevent the softening we observe in the current simulation results.

Despite this mismatch between the simulation and the experimental data loading orientations, we have retained the [100] and [111] loading orientations to examine the twinning behavior in the two sets of micropillar compression simulations. The twinning behavior, as calculated with the CDD crystal plasticity and twinning model we have presented in this chapter, aligns with our expectations for twinning behavior in the [100] orientation only under the compressive loading, as shown in Figure 5.6. The twinning volume fraction in this set of figures represents the twins in the pillar itself; although a small fraction of twins occur within the substrate, the volume fraction is sufficiently small to not be of interest to our comparison here.

In both the unaged and the 10,000 hours aged micropillar compression simulations, our results demonstrate, at the beginning of the deformation, the twinning evolution aligns with the trends we expected from our verification benchmarks, Section 5.2. During the early stages of deformation, the [100] orientations demonstrate clear twinning growth while twinning is essentially inactive in the [111] orientation 
micropillar. Once we reach a midpoint of the applied compressive displacement, the twins begin to grow in the thermally aged [111] loading orientation as well. Currently it is not clear if the activation of twins in the [111] orientation indicates an interaction of the thermally aged LRO precipitates with the twin dislocations through the glide dislocations or if the evolution of twins in this direction is an artifact introduced by the improper loading boundary condition. This issue warrants further investigation.

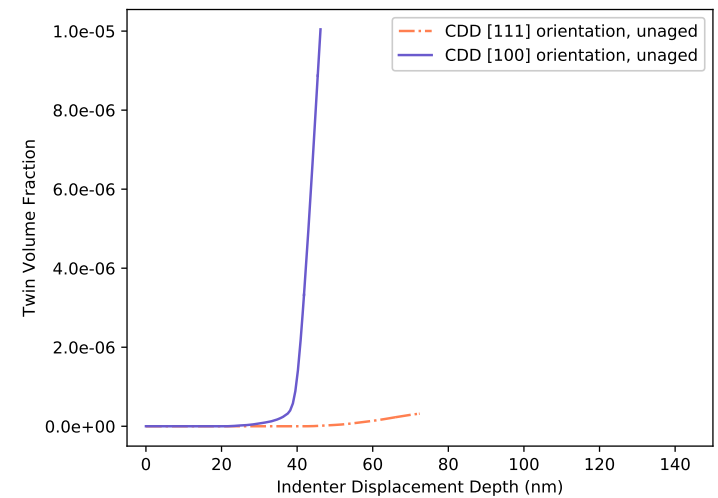

(a) Twinning volume fraction in the unaged micropillar simulations

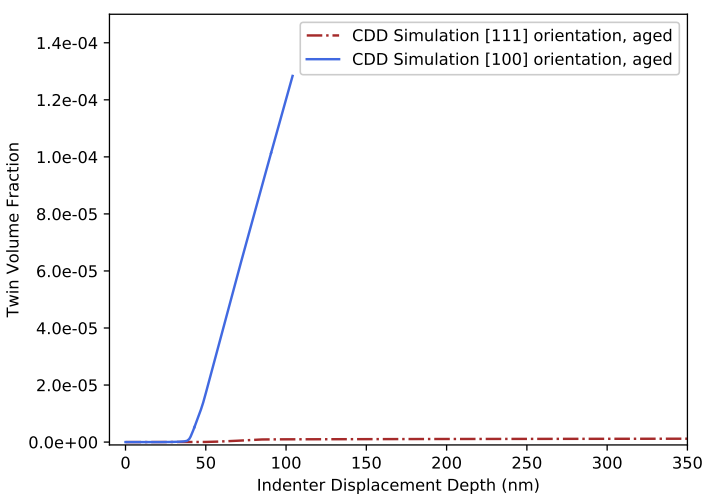

(b) Thermally aged micropillar simulation predictions of total twin volume fraction

Figure 5.6: In the unaged micropillar simulations the twinning behavior aligns with expectations for twins in only the [100] orientation; however, the thermally aged LRO precipitates appear to promote some twinning in both orientations of the micropillar simulations.

To understand and address the discrepancies between the experimental micropillar data and our simulation results, we begin by examining the evolution of the simulated microstructure. Careful inspection of the simulation glide dislocation evolution, twin 
growth, and system slip resistance will aid us in determining which physics are not fully captured by our crystal plasticity framework. We inspect two glide dislocation systems: one that is active in the micropillar, Figure 5.7a, and one that is mostly inactive in the pillar while being active in the region of the substrate near the pillar, Figure 5.7b.
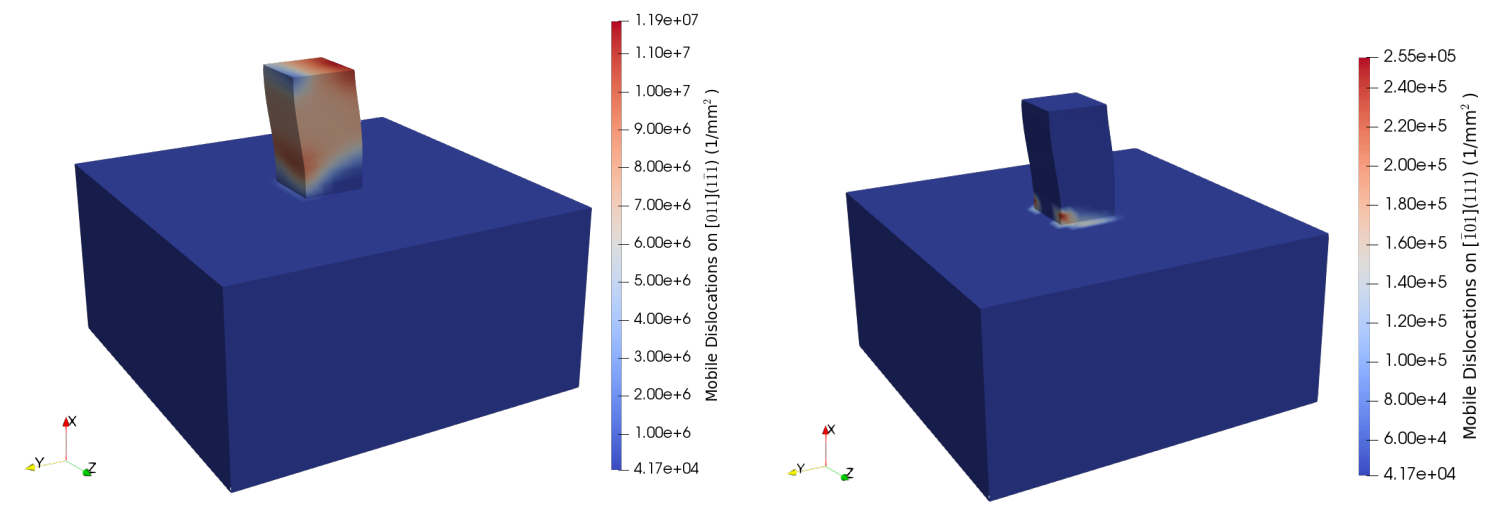

(a) Mobile dislocation density on the

(b) Mobile dislocation density on the [011](1111) slip system demonstrates growth [101](111) slip system in the base of the mionly in the micropillar. cropillar and in the substrate.

Figure 5.7: The evolution of mobile dislocations within the substrate at the base of the pillar demonstrate the importance of including the model of the substrate in our micropillar crystal plasticity simulations. Note the that scales used in Figures 5.7a and 5.7b are not equivalent.

The distribution of the mobile dislocations on the [101](111) slip system shows 
the importance of including the crystal plasticity model in the substrate. While the $[101](111)$ slip system is not active in the micropillar itself, the evolution of the dislocations on this system at the base of the micropillar will harden the overall response of the micropillar to the applied displacement.

\subsection{Conclusions and Future Recommendations}

In this chapter we have presented the implementation of a simplified twinning evolution model, suitable for use in our binary model $\mathrm{Ni}_{2} \mathrm{Cr}$ alloy. Although simple, this twinning model includes coupling among the glide dislocation evolution models and the twin growth model. This twinning model addition to the CDD crystal plasticity framework has been verified with a series of benchmark problems developed from conventional knowledge of twin formation under specific loading conditions in FCC materials. Additionally we have incorporated a hardening model specific for LRO precipitates, which form in thermally aged $\mathrm{Ni}_{2} \mathrm{Cr}$ and nickel-based alloys.

We have applied these twinning and LRO precipitate hardening models to the study of micropillar compression tests. The preliminary results of these simulations highlight the importance of modeling both the substrate and the micropillar components of the geometry with a crystal plasticity model. Nonetheless these results demonstrate that the crystal plasticity models we have presented here underpredict 
the micropillar response. We attribute this underprediction to a combination of an illposed loading boundary condition and an incomplete capture of the relevant physics for the $2 \mu \mathrm{m}$ diameter pillar. We propose as future work two additional models to address the underprediction.

Given the small size of these micropillar simulations, size dependent effects almost certainly play a role in explaining the high values of the force measured in the experiments and the calculated stress response. The addition of a geometrically necessary dislocation term, such as the one introduced in Chapter 4, may enable our simulation to better replicate the experimental measurements collected from the micropillar compression deformation. Furthermore the increase in work hardening observed in the thermally aged micropillar experiments motivates the future investigation of interaction mechanisms among the LRO precipitates and the glide and twin dislocations. 


\section{CHAPTER 6. DISLOCATION GLIDE VELOCITY MODEL COMPARISON}

A nuclear power plant reactor pressure vessel, one of the reactor components that is a focus of this thesis work, is subject to significant changes in temperature during refueling cycles. The temperatures range from around $300^{\circ} \mathrm{C}$ during normal operation to a room temperature around $30^{\circ} \mathrm{C}$ during the refueling shutdown [40]. Transient events could also raise the reactor temperature over a short period of time. Understanding how these changes in temperature affect the engineering scale properties of reactor pressure vessel steel is a key question which crystal plasticity models should help to address.

The increased development and use of physically based models in the crystal plasticity field has extended the ability to capture physical microstructure interactions with the additional expanded constitutive models. The trade off between more expanded physically based models and more condensed phenomenological models is the capture of physical events and interactions within the microstructure against the significantly larger number of material specific parameters which must be fit. Given the large numbers of material specific parameters in the physically based models, it is not trivial and is time consuming to individually determine fits for each of the material specific parameters [161]. Often engineering judgment must be applied to fit groups 
of parameters together to match limited experimental data.

Given the important role of dislocations in the inelastic deformation of metals as carriers of plasticity, as discussed in Section 2.2.3. Many physically based crystal plasticity models track the evolution of dislocation densities [10, 108, 55, 144], including our continuum dislocation dynamics (CDD) framework [177]. These models connect movement of the dislocations to the plastic shear through the Orowan equation, Eq (2.59). The force of the applied shear stress on these mobile dislocations is incorporated through the velocity term of the Orowan relation. The driving force component in the velocity equation is a function of the applied shear stress and the slip resistance of each individual slip system. This driving force is derived from the kinetic flow rule laws from other phenomenological crystal plasticity models $[159,85]$.

Within the field of crystal plasticity two main forms of a dislocation glide velocity have emerged: a power law form velocity model and an enthalpy-based velocity model $[161,99]$. The power law glide velocity model is constant across all temperature ranges while the enthalpy-based dislocation velocity model depends directly on temperature. As improvements to available computing power have mitigated the disparity in the computational requirements of these two models, the main difference in the application of these two models is in the number of material specific parameters used in each model.

To address the gap in the connection between engineering scale mechanical be- 
havior and changing temperature environments, our crystal plasticity model must include an appropriate dependence on temperature. In our work here we define the appropriate dependence on temperature to be a responsiveness of the calculated plasticity slip to temperature changes without the undue introduction of uncertainty from additional material specific parameters.

In this chapter we compare the use of the power law velocity equation, first introduced with the CDD crystal plasticity model, Section 2.3.1, to the enthalpy-based velocity model used by other groups studying BCC iron with crystal plasticity models $[144,28]$. We evaluate both dislocation glide velocity model for the ability to capture the effect of temperature on the results and for instabilities introduced by varying the velocity equation exponential parameters.

\subsection{Formulation of Dislocation Glide Velocity Equations}

We begin our comparison of the two dislocation glide velocity models with a review of the two equations. In the overviews of these two models we will discuss the potential advantages of each model and the material specific parameters associated with each model.

In all of the simulations with these two glide velocity models we have introduced the capability to model the anisotropic character of the slip system strength. We have 
previously shown anisotropic character of the slip system strength to be necessary to correctly capture closs slip in BCC $\alpha$ iron, Section 3.2.4, which is our intended material for our single crystal comparison studies in Section 6.2. As we will discuss in Section 6.1.1 and Section 6.1.2, the anisotropy is introduced into the slip system strengths by modifying the initial lattice friction quantity.

We start our overview with the power law glide velocity equation, which we have used in our models up to this point, before proceeding to review the enthalpy-based glide velocity equation.

\subsubsection{Power Law Glide Velocity Equation}

The power law dislocation glide mode implemented in the CDD model, Section 2.3.1, follows directly from the flow rule introduced by Rice $[159,146]$ through the Orowan relation

$$
\dot{\gamma}^{\alpha}=\dot{\gamma}_{o}\left|\frac{\tau^{\alpha}}{g^{\alpha}}\right|^{1 / m} \operatorname{sign}\left(\tau^{\alpha}\right) \Rightarrow v_{\text {glide }}^{\alpha}=v_{o}\left|\frac{\tau^{\alpha}}{g^{\alpha}}\right|^{1 / m} \operatorname{sign}\left(\tau^{\alpha}\right), \text { if } \tau^{\alpha} \geq g_{o}^{\alpha}
$$

where $v_{o}$ is the initial velocity. It follows through the Orowan relation that $v_{o}$ is a function of the reference strain rate, $\dot{\gamma}_{o}$

$$
\dot{\gamma}_{o}=\rho_{o}^{\text {mobile }} b v_{o} \Rightarrow v_{o}=\frac{\dot{\gamma}_{o}}{\rho_{o}^{\text {mobile } b}}
$$

where $\rho_{o}$ is the initial value of mobile dislocation density in the material at the start of the crystal plasticity simulation and $b$ is the Burgers vector. 
This power law velocity model therefore has two material specific parameters: the exponent value $m$ and the reference shear rate $\dot{\gamma}_{o}$. The value of the exponential constant is often held to a value around 0.010 [76] while the value of the reference strain rate varies from 0.001 to $0.1[76,101,177]$. Most crystal plasticity frameworks using the power law glide velocity equation have applied values of $m=0.012$ and $\dot{\gamma}_{o}=0.001[103]$.

The power law model offers a more robust approach to calculating the dislocation velocity and is less susceptible to numerical instabilities by virtue of its simplicity.

On the other hand this velocity model is not a function of temperature, which is known to impact dislocation velocity, Section 2.2.3. A dependence on temperature could be introduced by varying one or both parameters; however, this approach would require experimental data sets for calibration at each temperature of interest. This approach is not desirable nor is it always feasible.

\subsubsection{Enthalpy-based Glide Velocity Equation}

The second dislocation glide velocity model which we will consider we have termed the enthalpy-based velocity model. Because dislocation glide is thermally activated [69], enthalpy-based glide models account for temperature within an activation energy

term. The glide model we have chosen for this study was developed by Kothari and 
Anand, following Kocks' suggestions for the inclusion of an enthalpy type term to account for thermal bypass of small local obstacles in the dislocation path $[88,85]$.

The enthalpy-based model distinguishes between resistances from smaller local obstacles which can be bypassed with thermal fluctuations, thermal resistances, and the resistances caused by larger obstacles which require applied shear stress for dislocations to continue gliding, athermal resistances [88]. This decomposition of slip resistances is another difference from the power law glide velocity model, Eq (6.1), which contains all the slip resistance contributions within a single strength term. The mathematical formulation of the enthalpy-based dislocation glide velocity model is

$$
v_{\text {glide }}^{\alpha}=l_{g} \nu \cdot \exp \left(\frac{-\Delta F}{k T}\left(1-\left(\frac{\left|\tau^{\alpha}\right|-g_{a}^{\alpha}}{g_{t}^{\alpha}}\right)^{p}\right)^{q}\right) \text { if } \tau^{\alpha} \geq g_{a}^{\alpha}
$$

where $l_{g}$ is a constant mean free glide length, $\nu$ is the jump frequency of the dislocations, $\Delta F$ is the stress free activation energy, $k$ is the Boltzmann constant, $T$ is the temperature in Kelvin, $\tau^{\alpha}$ is the applied shear stress, $g_{a}^{\alpha}$ and $g_{t}^{\alpha}$ are the aforementioned athermal and thermal resistances, and $p$ and $q$ are exponential function shape parameters, respectively [144, 28].

This enthaply based velocity mode has six different material specific parameters, excluding the athermal slip resistance term. The pre-exponential terms $l_{g}$ and $\nu$ are related through a similar application of Orowan's relation as we used in the case of 
the power law velocity model, Eq (6.2). Here the Orowan's relation has the form

$$
\dot{\gamma}_{o}=\rho_{o} b\left(l_{g} \nu\right)
$$

such that the mean free glide length, $l_{g}$, and the jump frequency, $\nu$, are similar to the initial velocity value in the power law model. A constant value for $l_{g}$ is generally assumed so that the jump frequency can be estimated from Eq (6.4). The value of the $\dot{\gamma}_{o}$ term is the same as used in the power law velocity model [144]. The activation energy has the form

$$
\Delta F=f \mu b^{3}
$$

where $f$ is a coeffiecent generally between 0.05 and 2.0 [88], $\mu$ is the material shear modulus, and $b$ is the Burgers vector.

The separation of the slip system resistances into the thermal and athermal components requires an adjustment to the summed slip system strengths in Eq (2.70). Following Chakraborty and Biner we have attributed hardening due to dislocation forests, Eq (2.71), and irradiation defects, Eq (2.72), to the athermal resistance, $g_{a}^{\alpha}$ term.

Because of the importance of the anisotropy in the calculation of the cross slip, Section 3.2.4, we have implemented an anistropic factor for the Peierls stress into the calculation of the thermal resistance, $g_{t}^{\alpha}$. The athermal resistance is described as being akin to the Peierls stress, or lattice friction, of the crystal lattice [144] and thus is the most appropriate term in which to include the anisotropy effect. 
The final two material specific terms in the enthalpy-based velocity equation are the shape function parameters; these terms impact the velocity equation by determining the shape of the activation enthalpy and applied shear stress to slip resistance ratio curves [88]. The following bounds on these exponential constants are often suggested in literature to be

$$
\begin{aligned}
& 0 \leq p \leq 1 \\
& 1 \leq q \leq 2
\end{aligned}
$$

although most applications of these terms tend toward the lower end of both ranges $[88,10]$.

The temperature dependence of the activation free energy enthalpy term offers a direct method to incorporate the effects of temperature on the crystal plasticity model. With careful fitting of the many material specific parameters, the enthalpy velocity equation can be applied to a wide range of temperatures. For these reason this enthalpy-based velocity model, Eq 6.3, or a similar velocity model, is often preferred for use in current crystal plasticity frameworks [161].

We do, however, express a measure of concern about the large number of parameters which must be fit in the enthalpy-based velocity model. In addition to the challenge of fitting all of the parameters, there also exists a potential to introduce numerical instabilities into the model with different material parameter selections. 


\subsection{Single Crystal $\alpha$ Iron Simulations}

To explore these potential issues with temperature range and material parameter stability we have conducted a set of single crystal simulations for an $\alpha$ iron $\mathrm{BCC}$ material. In the first set of simulations we have maintained the velocity equation constants from previous work while varying the temperatures. In the second set of simulations we have held the temperature constant while varying the exponential parameters in each model. We have chosen to focus on the exponential parameters because of the similarity in the pre-exponential factors of each model, recall Eq (6.2) and Eq (6.4), and because of the larger probability for the exponential terms to introduce numerical instability.

In all of these simulations we have retained the CDD dislocation evolution models, Eq (2.67), Eq (2.68), and Table 2.4 for glide dislocations only and have excluded the models for geometrically necessary dislocations and twin dislocations. We have changed only the dislocation glide velocity equation. We have also retained all of the elastic constants and material parameters for BCC $\alpha$ iron, listed in Table 6.1.

As in the set of $\alpha$ iron single crystal simulations we performed to fit the dislocation evolution parameters, we have conducted these simulations on a $1 \mathrm{~mm}^{3}$ cube with a $216\left(6^{3}\right)$ Hex8 element mesh with symmetry boundary conditions. 
Table 6.1: Constant material parameter values used in the single crystal $\alpha$-Fe simulations for the comparison of dislocation glide velocity models.

\begin{tabular}{|c|c|c|}
\hline Parameter & Value & Description \\
\hline$C_{11}$ & $242 \times 10^{3} \mathrm{MPa}$ & Elastic constant, [65] \\
\hline$C_{12}$ & $150 \times 10^{3} \mathrm{MPa}$ & Elastic constant, [65] \\
\hline$C_{44}$ & $112 \times 10^{3} \mathrm{MPa}$ & Elastic constant, [65] \\
\hline$\mu$ & $80 \times 10^{3} \mathrm{MPa}$ & Shear modulus, [65] \\
\hline$b$ & $2.48 \times 10^{-7} \mathrm{~mm}$ & Burgers vector,[65], Eq (2.59) \\
\hline$\alpha_{m b h}$ & 0.4 & Dispersed barrier coefficient, Eq (2.71) \\
\hline$\Omega^{\alpha \alpha}$ & 1.0 & Self-hardening coefficient, Eq (2.71) \\
\hline$\Omega^{\alpha \beta}$ & 0.2 & Latent-hardening coefficient, Eq (2.71) \\
\hline$\omega^{\alpha \alpha}, \omega^{\alpha \beta}$ & 1.0 & Mean free glide path, Eq (2.69) \\
\hline$R_{c}$ & $15 b \mathrm{~mm}$ & Annihilation radius of capture, Table 2.4 \\
\hline$\tau^{*}$ & $4 \times 10^{-3} \cdot \mu$ & Critical cross slip stress, Eq (3.3) \\
\hline$V_{a}$ & $20 b^{3}$ & Volume for dislocation cross slip, Eq (3.3) \\
\hline$k$ & $1.38065 \times 10^{-20} \frac{\mathrm{MPa}-\mathrm{mm}^{3}}{\mathrm{~K}}$ & Bolztmann constant, Eq (3.3) \\
\hline$\tau_{p s}$ & $15 \mathrm{MPa}$ & Isotropic Peierls strength, Eq (3.2) \\
\hline
\end{tabular}

We have loaded the single crystal cube in tension along the [100] direction with a displacement rate of $3.3 \times 10^{-4} \mathrm{~mm} / \mathrm{s}$. Correspondingly we have used the disloca- 
tion evolution parameters for the [100] direction. These parameters and the initial dislocation density values are listed in Table 6.2.

Table 6.2: Values of the dislocation evolution parameters used in the simulations comparing dislocation glide velocity equations. These parameters were fit to single crystal $\alpha$-Iron experimental data in Section 3.2.1 for the CDD dislocation evolution model, Eq (2.67) and Eq (2.68) and are appropriate for loading in the [100] direction.

\begin{tabular}{lcl}
\hline Dislocation Evolution Interaction & Constant & Value \\
\hline Frank-Read generation & $\alpha_{1}$ & 0.03 \\
Mobile-mobile annihilation & $\alpha_{2}$ & 0.5 \\
Dislocation locking & $\alpha_{3}$ & 0.002 \\
Locked dislocations freed & $\alpha_{4}$ & 0.002 \\
Cross slip & $\alpha_{5}$ & 0.015 \\
Immobile-mobile annihilation & $\alpha_{6}$ & 1.0 \\
Initial total mobile dislocation density & $\rho_{o}^{\text {mobile }}$ & $2.5 \times 10^{5} 1 / \mathrm{mm}^{2},[99]$ \\
Initial total immobile dislocation density & $\rho_{o}^{\text {immobile }}$ & $2.5 \times 10^{5} 1 / \mathrm{mm}^{2},[99]$ \\
\hline
\end{tabular}




\subsubsection{Variation of Temperature Comparisons}

In this section we explore the response of the two dislocation glide velocity models, Eq (6.1) and Eq (6.3), to changes in temperature. We expect the predicted stressstrain response to change with increasing temperature. While the enthalpy-based velocity equation depends directly on temperature, the only sensitivity to temperature in the crystal plasticity simulations using the power law velocity model comes through the cross slip term, Eq (3.3). Although the terms of the elasticity tensor are also affected by changes in the temperature, for the purposes of this study we have held those parameters constant at the values given in Table 6.1. In the results of this temperature study, Section 6.2.1, we compare the responses of the crystal plasticity simulations to determine if the response to changing temperature is adequate with both velocity models or only with the enthalpy-based glide velocity model.

\section{Temperature Variations Used in the Simulation Set}

In this temperature variation study and in the exponential constants variation study, Section 6.2.2, we have used the set of dislocation glide velocity equation parameters from previous works: we apply the same parameters for the power law velocity as we used in Chapter 3 and we use the parameters determined by Patra and McDowell for the enthalpy-based velocity model [145]. These constants are given in Table 6.3 and Table 6.4 . 
Table 6.3: Parameter values for the power law dislocation glide velocity model, Eq (6.1) in the temperature variation comparison of dislocation glide velocity model, taken from our work in Section 3.2.1. The initial dislocation glide velocity is calculated through Eq (6.2) from a reference shear rate of $4.0 \times 10^{-2}$ [101], as given in Table 3.1.

\begin{tabular}{lcl}
\hline Dislocation Glide Velocity Term & Constant & Value \\
\hline Initial dislocation glide velocity & $v_{o}$ & $0.645 \mathrm{~mm} / \mathrm{s}$ \\
Exponential constant & $m$ & $0.012[101]$ \\
\hline
\end{tabular}

Table 6.4: Parameter values for the enthalpy-based dislocation glide velocity model, Eq (6.1) in the temperature variation comparison of dislocation glide velocity model, from [28].

\begin{tabular}{lcl} 
Dislocation Glide Velocity Term & Constant & Value \\
\hline Constant mean free glide length & $l_{g}$ & $1.5 \times 10^{-6} \mathrm{~mm}$ \\
Jump frequency & $\nu$ & $5.396 \times 10^{9}$ \\
Stress free activation energy & $\Delta F$ & $0.35 \mu \mathrm{b}^{3} \frac{\mathrm{MPa}}{\mathrm{mm}^{3}}$ \\
Boltzmann constant & $k$ & $1.38065 \times 10^{-20} \frac{\mathrm{MPa}-\mathrm{mm}^{3}}{\mathrm{~K}}$ \\
Inner exponential shape parameter & $p$ & $0.28[145]$ \\
Outer exponential shape parameter & $q$ & $1.34[145]$ \\
\hline
\end{tabular}


We have selected a set of different temperatures which span the expected operating temperatures for a reactor pressure vessel across a refueling cycle: $25^{\circ} \mathrm{C}$ to $300^{\circ} \mathrm{C}$ [40]. The full set of temperatures used in this study is given in Table 6.5.

Table 6.5: Set of temperature values, spanning the typical range of temperatures experienced by the reactor pressure vessel for normal operating conditions to shutdown [40], used in the temperature study portion of the dislocation glide velocity model comparison.

\begin{tabular}{cc}
\hline Simulation Run & Temperature \\
\hline $\mathrm{T} 1$ & $25^{\circ} \mathrm{C}$ \\
$\mathrm{T} 2$ & $75^{\circ} \mathrm{C}$ \\
$\mathrm{T} 3$ & $150^{\circ} \mathrm{C}$ \\
$\mathrm{T} 4$ & $225^{\circ} \mathrm{C}$ \\
$\mathrm{T} 5$ & $300^{\circ} \mathrm{C}$ \\
\hline
\end{tabular}

\section{Results of Temperature Variations}

Intuitively we expect increases in the temperature to produces a softer stress response: that is, for a given strain the stress from a sample at a higher temperature will be smaller than the stress in a sample tested a a lower temperature. Experimental data, from tensile tests conducted on single crystals of copper and aluminum at a wide range of temperatures, also assert that a metal material should resist deformation less at higher temperatures $[178,67]$. Both of these works show significant reductions in 
the measured stress over a prescribed strain with increasing sample temperature. On the mesoscale, individual dislocations can travel further at higher temperatures under an applied stress, thus the crystal is able to undergo more plastic deformation.

To capture the influence temperature has on the stress response predicted by crystal plasticity simulations, we rely in this study on the dislocation glide velocity model and, to a lesser extent, the cross slip term in the dislocation evolution term, as discussed in Section 6.1. Over the range of temperatures specified in Table 6.5, we evaluated the two dislocation glide velocity models, Eq (6.1) and Eq (6.3), on their ability to capture the trend of decreasing stress with increasing temperature. We present the results from the power law glide velocity model in Figure 6.1 and the results from the enthalpy-based dislocation glide velocity model in Figure 6.2.

We observe only a minimal variation of the stress response to changes in temperature from the power law dislocation glide velocity model, Figure 6.1a. Since the variation in the stress response with different simulation temperatures occurs in the later stages of deformation, we attribute the temperature sensitivity to the cross slip term in the dislocation evolution model, Eq 3.4. The increased temperature increases the cross slip activity and thus softens the effective stress response of the single crystal simulation. Addition evidence of the increased cross slip activity is observed in the evolution of the mobile dislocations on active slip systems, Figure 6.1b. With increasing simulation temperature the mobile dislocation density decreases as more 


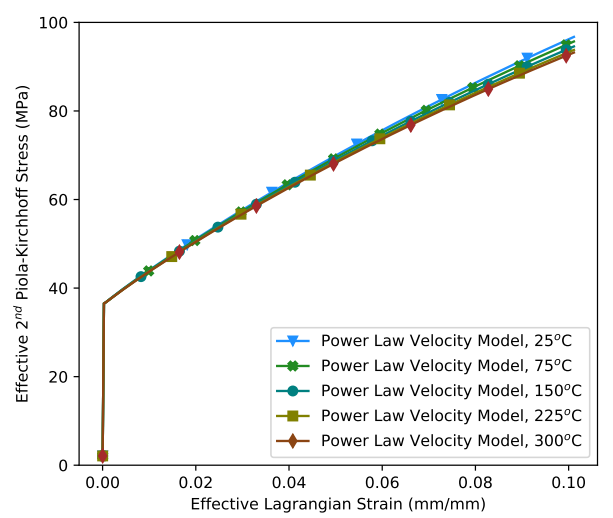

(a) Response of the effective stress at vari-

ous different temperatures

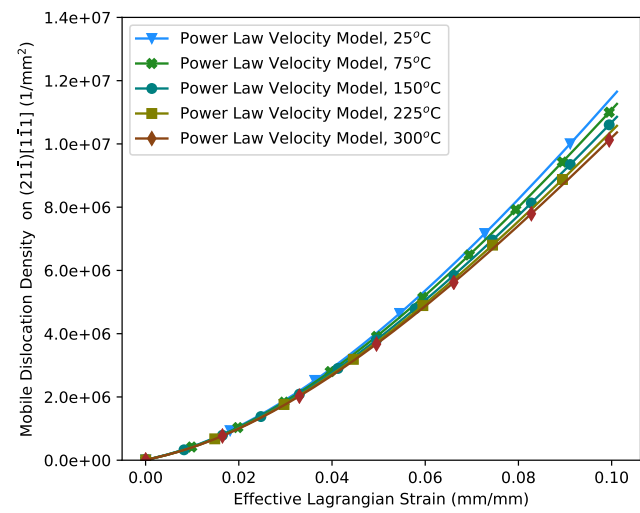

(b) Mobile dislocation evolution on $(21 \overline{1})[1 \overline{1} 1]$, an active slip system.

Figure 6.1: The power law glide velocity model does not demonstrate large changes in the stress response or in the mobile dislocation evolution to changes in temperature; however, the observed changes are consistent with expectations for increasing temperature. The power law glide velocity model consistently demonstrates lower stresses and lower mobile dislocation densities with increasing temperature values as expected.

mobile dislocation cross slip away from the softer $\{112\}$ slip systems to the harder $\{110\}$ systems. We have selected the $(21 \overline{1})[1 \overline{1} 1]$ slip system as a representative of the several active slip systems with the [100] loadig direction.

Both of these trends in Figure 6.1 are in line with the trends observed among the experimental data; however, the power law velocity simulation stress response is notably less sensitive to temperature variations than the experimental measurements. While the power law glide velocity model is not directly responsible for these simu- 
lation trends, crucially it does not interfere with the temperature sensitive response of other models within the CDD crystal plasticity framework. We further suggest a potential modification to the power law dislocation glide velocity model in Section 6.2.3 to introduce a direct dependence on temperature rather than relying solely on the cross slip term to provide temperature dependence.

The response of the enthalpy-based dislocation glide velocity model over the set of temperatures listed in Table 6.5 is shown in Figure 6.2. The variation of the stress response over the first three temperatures in Table 6.5 aligns with our expectations: each increase in temperature produces a significant drop in the stress response. For the highest two temperatures, howevever, we observe a troubling trend: the stress response at these higher temperatures demonstrates a nonphysical hardening of the effective stress response. Both enthalpy-based velocity model simulations at $225^{\circ} \mathrm{C}$ and $300^{\circ} \mathrm{C}$ predict higher effective stresses at 0.1 effective strain than the simulation at $150^{\circ} \mathrm{C}$. As seen in Figure $6.2 \mathrm{a}$, the $225^{\circ} \mathrm{C}$ simulation stress curve crosses the $150^{\circ} \mathrm{C}$ curve at approximately $0.9 \mathrm{~mm} / \mathrm{mm}$ strain, and the $300^{\circ} \mathrm{C}$ simulation stress response crosses the stress curve from the $150^{\circ} \mathrm{C}$ simulation at $0.8 \mathrm{~mm} / \mathrm{mm}$ strain.

This same interference from the enthalpy-based velocity model is also evident in the mobile dislocation evolution on active slip systems, Figure 6.2b. The mobile dislocation evolution at the lower temperatures follows the expected trends with successive lowering of the densities. At the higher simulation temperatures the mobile 
dislocation density evolution also demonstrates similar nonphysical hardening trends as seen in the stress response.

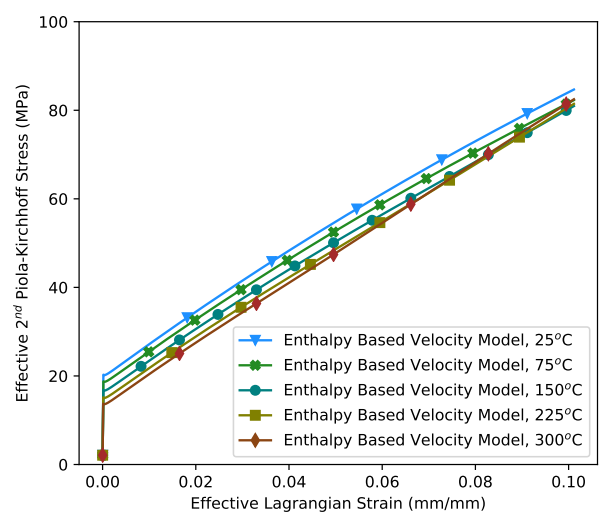

(a) Response of the effective stress at vari-

ous different temperatures

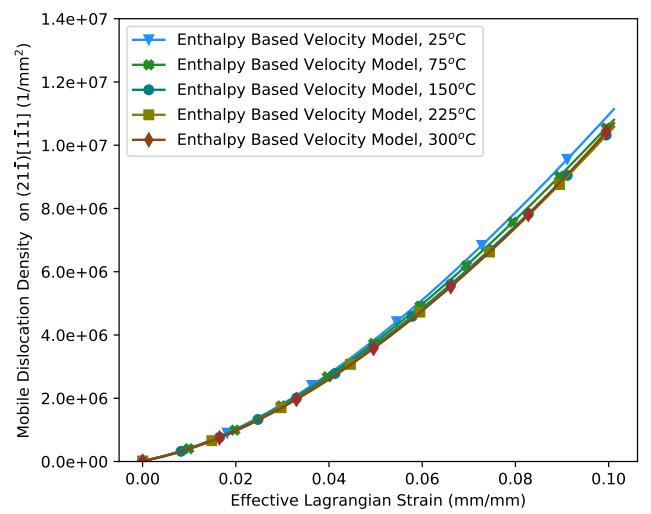

(b) Mobile dislocation evolution on $(21 \overline{1})[1 \overline{1} 1]$, an active slip system.

Figure 6.2: The enthalpy-based glide velocity model demonstrates a noticeable change in the stress response and in the mobile dislocation evolution with increasing temperature. The trend of these changes is not consistent across the range or temperatures nor consistent with expectations based on experimental observations: at the two higher temperatures, $225^{\circ} \mathrm{C}$ and $300^{\circ} \mathrm{C}$, the enthalpy-based velocity model predicts a non-physical increase in the strength of the material compared to the simulation at $150^{\circ} \mathrm{C}$, accompanied by a similar increase in mobile dislocation accumulation.

These inconsistent responses of the enthalpy-based velocity model crystal plasticity simulations are indicative of the limited suitability of this model for predictive simulations conducted over a range of temperatures. While the temperature sensitive 
response at lower temperatures is better able to capture the experimentally observed softening, the nonphysical hardening at the higher temperatures in our study range increases the probability of unreliable predictions when using this velocity model. While both dislocation glide velocity models will require recalibration to properly capture the impact of temperature, simulations which employ the enthalpy-based velocity model will require additional testing to determine when the nonphysical trends observed in Figure 6.2 begin.

Given the necessary process of recalibrating both dislocation glide velocity models to capture the effects of increasing temperature, we explore the impact of varying the exponential constants in both velocity models in the next section.

\subsubsection{Variation of Exponential Velocity Equation Constants}

In this section we investigate the sensitivity of the two dislocation glide velocity models, Eq (6.1) and Eq (6.3), to changes in the exponential parameters in each equation. We have explored the range of allowable exponential values at a constant temperature and evaluated the results for stability. A wide set of parameter variations which produce stable results provides confidence in the future ability to calibrate a dislocation glide velocity model to a new crystalline metal. We have elected to vary only the exponential terms in this study for two reasons: 
- The pre-exponential coefficient terms in the power law velocity equation and in the enthalpy-based velocity equation are both related to the same reference shear rate through the Orowan relation, Eq (6.2) and Eq (6.4). Thus these two pre-exponential terms are in essence equivalent.

- The exponential terms, which in both cases act on the applied shear stress to the slip resistance ratio terms, have the highest potential to introduce numerical instability into the velocity equations.

Varying the exponential constants, $m$ in the power law velocity model, Eq (6.1), and the combination of $p$ and $q$ in the enthalpy-based velocity model, Eq (6.3), enables us to perform a set of comparable variations of both models.

\section{Parameter Variations Used in the Simulation Set}

Both glide velocity modes are generally presented in literature with only a small variation in the exponential parameters: $m$, from the power law velocity model, varies from $0.010-0.012[76,103]$ while $p$ and $q$ from the enthalpy-based model are often assigned values between $0.1-0.3$ and 1.1 - 1.3, respectively [88, 10, 144, 28].

We have selected a variation of the enthalpy-based velocity model exponential constants of 0.1 increments in the lower half of the allowable ranges, Eq (6.6) at two constant temperatures: $25^{\circ} \mathrm{C}$ and $150^{\circ} \mathrm{C}$. We have applied a similar set of variations to the exponential coefficients from the power law velocity model. Since the power 
Table 6.6: Variations of the exponential constant in the power law dislocation glide velocity model, Eq (6.1) used in the coefficient perturbation study

\begin{tabular}{ccc}
\hline Simulation Run & Exponential Constant $(m)$ & Temperature \\
\hline P1 & 0.010 & $25^{\circ} \mathrm{C}$ \\
P2 & 0.015 & $25^{\circ} \mathrm{C}$ \\
P3 & 0.020 & $25^{\circ} \mathrm{C}$ \\
P4 & 0.025 & $25^{\circ} \mathrm{C}$ \\
P5 & 0.030 & $25^{\circ} \mathrm{C}$ \\
P6 & 0.035 & $25^{\circ} \mathrm{C}$ \\
P7 & 0.040 & $25^{\circ} \mathrm{C}$ \\
P8 & 0.045 & $25^{\circ} \mathrm{C}$ \\
P9 & 0.050 & $25^{\circ} \mathrm{C}$ \\
\hline
\end{tabular}

law model does not directly depend on temperature we have run these simulations at a temperature of only $25^{\circ} \mathrm{C}$. The variation of the exponential constants for the power law velocity equation are given in Table 6.6 and the variations of the enthalpybased velocity equation are given in Table 6.7 and Table 6.8. We retain the values for the remainder of the parameters in the velocity equations from the temperature study as given in Table 6.3 for the power law velocity model, and Table 6.4 for the enthalpy-based velocity model. 
Table 6.7: Variation of exponential constants in the enthalpy-based dislocation glide velocity model, Eq (6.3), at a constant temperature of $25^{\circ} \mathrm{C}$, used in the coefficient study

\begin{tabular}{|c|c|c|c|}
\hline \multirow[t]{2}{*}{ Simulation Run } & \multicolumn{2}{|c|}{ Exponential Constants } & \multirow[t]{2}{*}{ Temperature } \\
\hline & $(p)$ & $(q)$ & \\
\hline $\mathrm{C} 1$ & 0.1 & 1.1 & $25^{\circ} \mathrm{C}$ \\
\hline $\mathrm{C} 2$ & 0.1 & 1.2 & $25^{\circ} \mathrm{C}$ \\
\hline C3 & 0.1 & 1.3 & $25^{\circ} \mathrm{C}$ \\
\hline $\mathrm{C} 4$ & 0.1 & 1.4 & $25^{\circ} \mathrm{C}$ \\
\hline C5 & 0.1 & 1.5 & $25^{\circ} \mathrm{C}$ \\
\hline C6 & 0.2 & 1.1 & $25^{\circ} \mathrm{C}$ \\
\hline $\mathrm{C} 7$ & 0.2 & 1.2 & $25^{\circ} \mathrm{C}$ \\
\hline $\mathrm{C} 8$ & 0.2 & 1.3 & $25^{\circ} \mathrm{C}$ \\
\hline C9 & 0.2 & 1.4 & $25^{\circ} \mathrm{C}$ \\
\hline C10 & 0.2 & 1.5 & $25^{\circ} \mathrm{C}$ \\
\hline C11 & 0.3 & 1.1 & $25^{\circ} \mathrm{C}$ \\
\hline C12 & 0.3 & 1.2 & $25^{\circ} \mathrm{C}$ \\
\hline C13 & 0.3 & 1.3 & $25^{\circ} \mathrm{C}$ \\
\hline C14 & 0.3 & 1.4 & $25^{\circ} \mathrm{C}$ \\
\hline C15 & 0.3 & 1.5 & $25^{\circ} \mathrm{C}$ \\
\hline
\end{tabular}


Table 6.7: Variation of exponential constants in the enthalpy-based dislocation glide velocity model, $\mathrm{Eq}(6.3)$, at a constant temperature of $25^{\circ} \mathrm{C}$, used in the coefficient variation study (continued)

\begin{tabular}{cccc} 
Simulation Run & \multicolumn{2}{c}{ Exponential Constants } & Temperature \\
& $(p)$ & $(q)$ & \\
\hline $\mathrm{C} 16$ & 0.4 & 1.1 & $25^{\circ} \mathrm{C}$ \\
$\mathrm{C} 17$ & 0.4 & 1.2 & $25^{\circ} \mathrm{C}$ \\
$\mathrm{C} 18$ & 0.4 & 1.3 & $25^{\circ} \mathrm{C}$ \\
$\mathrm{C} 19$ & 0.4 & 1.4 & $25^{\circ} \mathrm{C}$ \\
$\mathrm{C} 20$ & 0.4 & 1.5 & $25^{\circ} \mathrm{C}$ \\
$\mathrm{C} 21$ & 0.5 & 1.1 & $25^{\circ} \mathrm{C}$ \\
$\mathrm{C} 22$ & 0.5 & 1.2 & $25^{\circ} \mathrm{C}$ \\
$\mathrm{C} 23$ & 0.5 & 1.3 & $25^{\circ} \mathrm{C}$ \\
$\mathrm{C} 24$ & 0.5 & 1.4 & $25^{\circ} \mathrm{C}$ \\
$\mathrm{C} 25$ & 0.5 & 1.5 & $25^{\circ} \mathrm{C}$ \\
\hline
\end{tabular}

These variations on the exponential constants of the two dislocation glide velocity models allow us to better anticipate difficulties which may arise in the recalibration of the glide velocity models for higher temperatures which we discuss in Section 6.2.1. 
Significant sensitivity of a model to changes in the exponential parameters indicates that additional care will be required before using that model in a higher temperature simulation.

Table 6.8: Variation of exponential constants in the enthalpy-based dislocation glide velocity model, Eq (6.3), at a constant temperature of $150^{\circ} \mathrm{C}$, used in the coefficient study

\section{Simulation Run Exponential Constants Temperature}

\begin{tabular}{cccc} 
& $(p)$ & $(q)$ & \\
\hline $\mathrm{C} 26$ & 0.1 & 1.1 & $150^{\circ} \mathrm{C}$ \\
$\mathrm{C} 27$ & 0.1 & 1.2 & $150^{\circ} \mathrm{C}$ \\
$\mathrm{C} 28$ & 0.1 & 1.3 & $150^{\circ} \mathrm{C}$ \\
$\mathrm{C} 29$ & 0.1 & 1.4 & $150^{\circ} \mathrm{C}$ \\
$\mathrm{C} 30$ & 0.1 & 1.5 & $150^{\circ} \mathrm{C}$ \\
$\mathrm{C} 31$ & 0.2 & 1.1 & $150^{\circ} \mathrm{C}$ \\
$\mathrm{C} 32$ & 0.2 & 1.2 & $150^{\circ} \mathrm{C}$ \\
$\mathrm{C} 33$ & 0.2 & 1.3 & $150^{\circ} \mathrm{C}$ \\
$\mathrm{C} 34$ & 0.2 & 1.4 & $150^{\circ} \mathrm{C}$ \\
$\mathrm{C} 35$ & 0.2 & 1.5 & $150^{\circ} \mathrm{C}$ \\
$\mathrm{C} 36$ & 0.3 & 1.1 & $150^{\circ} \mathrm{C}$ \\
$\mathrm{C} 37$ & 0.3 & 1.2 & \\
\hline
\end{tabular}


Table 6.8: Variation of exponential constants in the enthalpy-based dislocation glide velocity model, $\mathrm{Eq}(6.3)$, at a constant temperature of $150^{\circ} \mathrm{C}$, used in the coefficient variation study (continued)

\section{Simulation Run Exponential Constants Temperature}

\begin{tabular}{|c|c|c|c|}
\hline & $(p)$ & $(q)$ & \\
\hline C38 & 0.3 & 1.3 & $150^{\circ} \mathrm{C}$ \\
\hline C39 & 0.3 & 1.4 & $150^{\circ} \mathrm{C}$ \\
\hline $\mathrm{C} 40$ & 0.3 & 1.5 & $150^{\circ} \mathrm{C}$ \\
\hline C41 & 0.4 & 1.1 & $150^{\circ} \mathrm{C}$ \\
\hline $\mathrm{C} 42$ & 0.4 & 1.2 & $150^{\circ} \mathrm{C}$ \\
\hline $\mathrm{C} 43$ & 0.4 & 1.3 & $150^{\circ} \mathrm{C}$ \\
\hline $\mathrm{C} 44$ & 0.4 & 1.4 & $150^{\circ} \mathrm{C}$ \\
\hline C45 & 0.4 & 1.5 & $150^{\circ} \mathrm{C}$ \\
\hline $\mathrm{C} 46$ & 0.5 & 1.1 & $150^{\circ} \mathrm{C}$ \\
\hline $\mathrm{C} 47$ & 0.5 & 1.2 & $150^{\circ} \mathrm{C}$ \\
\hline C48 & 0.5 & 1.3 & $150^{\circ} \mathrm{C}$ \\
\hline C49 & 0.5 & 1.4 & $150^{\circ} \mathrm{C}$ \\
\hline C50 & 0.5 & 1.5 & $150^{\circ} \mathrm{C}$ \\
\hline
\end{tabular}




\section{Results of Exponential Constants Variations}

In this section we present the results of varying the exponential constants in both the power law velocity model and in the enthalpy-based velocity model. Given the large number of parameter variations considered in the case of the enthalpy-based model we have sorted the results for this velocity model into five separate figures by the outer exponential shape function parameter, $p$, in Figures 6.4, 6.5, 6.6, and 6.7. The relative simplicity of the power law velocity model allows us to coalesce the results into a single graph, Figure 6.3. As in the temperature study results, Section 6.2.1, we present the effective stress response and the mobile dislocation evolution on the active $(21 \overline{1})[1 \overline{1} 1]$ slip system from these crystal plasticity simulations.

The power law velocity model demonstrates an acceptable and stable response to changes in the exponential parameter $m$. We observe equivalent drops in the stress response with increasing values of the exponent, Figure 6.3a. The impact of the exponential variation on the evolution of mobile dislocations is limitied, Figure 6.3b, with the various mobile dislocation density curves being difficult to distinguish from one another. We note that for higher values of the exponential constant $m$, the stress curve demonstrates a dip at the onset of plasticity: this behavior is noticeable for exponential constant values above 0.030 .

The stability in the responses of all the exponential constant values listed in Table 6.6 gives us confidence that the power law glide velocity model could be easily 
recalibrated for higher temperatures. Furthermore these results indicate that the simulation results from the recalibrated power law velocity model will be reliable.

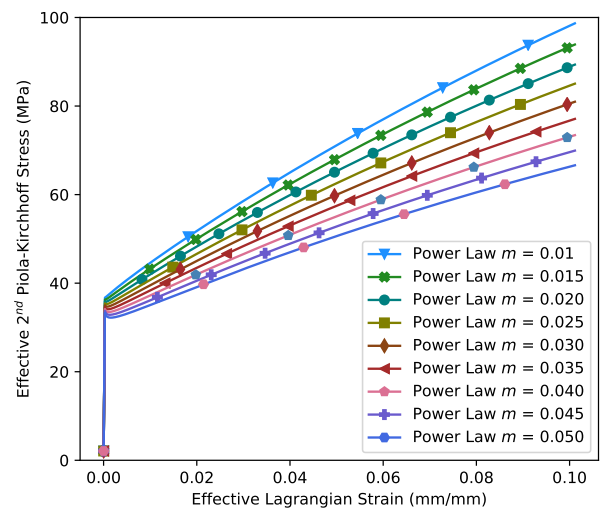

(a) Response of the effective stress to changes in the velocity model exponential constant at $25^{\circ} \mathrm{C}$

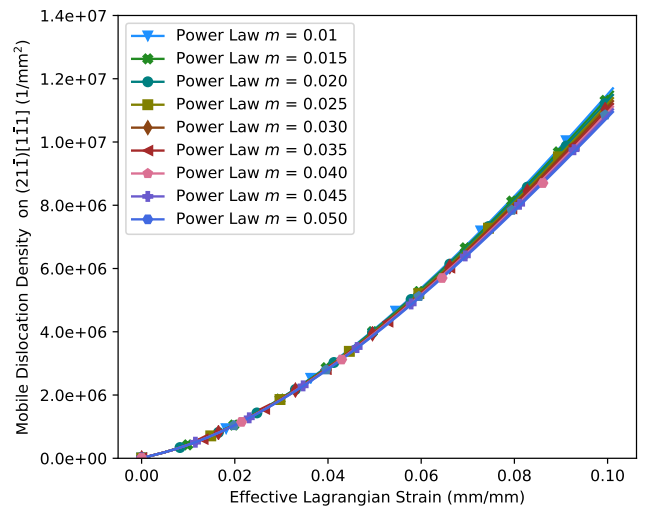

(b) Evolution of the mobile dislocation density on $(21 \overline{1})[1 \overline{1} 1]$, one of the active slip systems, at $25^{\circ} \mathrm{C}$

Figure 6.3: Variation of the stress response and mobile dislocation evolution, on an active slip system, to changes in the value of the power law velocity exponential constant, $m$, as given in Table 6.6, demonstrate a consistent reduction in both quantities with increasing values of the exponential constant.

The sheer number of simulations required to test the enthalpy-based velocity model is an indication of the variability inherent in this model. While variability can enable a model to capture a greater number of physical events it can also introduce more uncertainty into the results. In this study we evaluate this inherent variability for the potential of unstable or nonphysical results. 


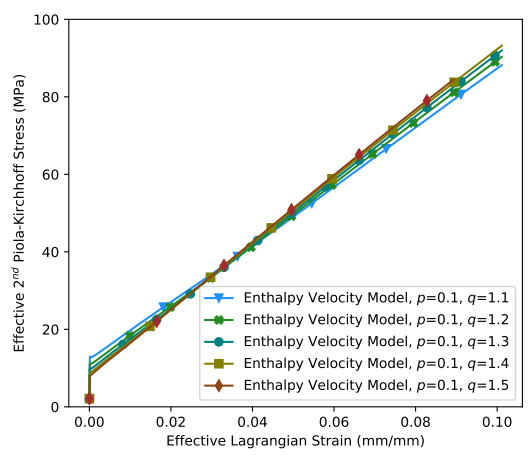

(a) Variations with $p=0.1$ at $25^{\circ} \mathrm{C}$

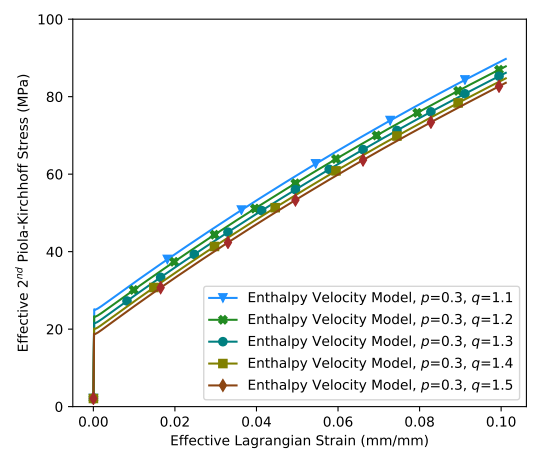

(c) Variations with $p=0.3$ at $25^{\circ} \mathrm{C}$

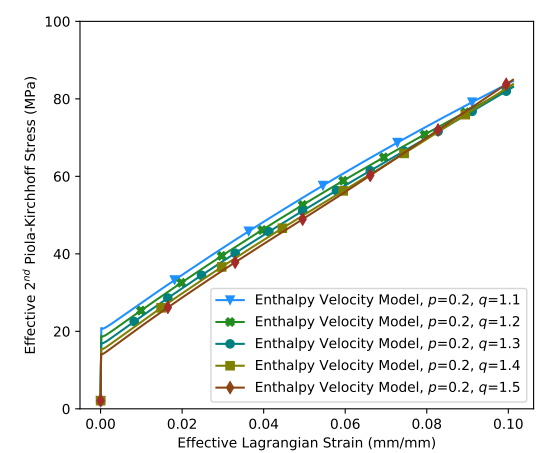

(b) Variations with $p=0.2$ at $25^{\circ} \mathrm{C}$

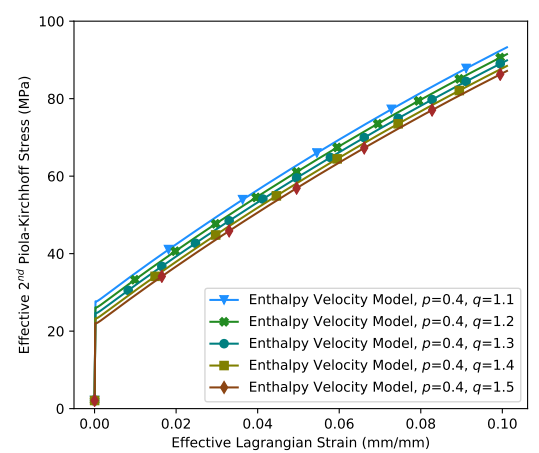

(d) Variations with $p=0.4$ at $25^{\circ} \mathrm{C}$

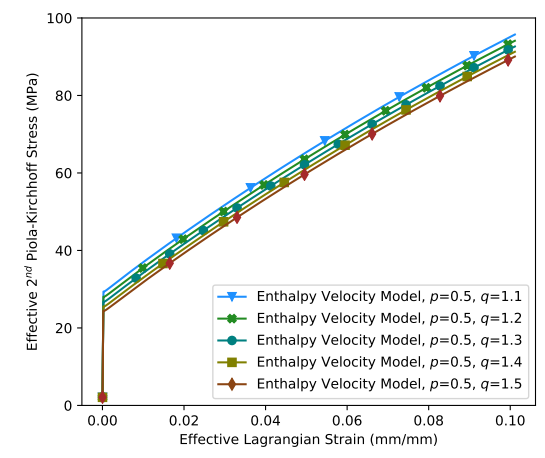

(e) Variations with $p=0.5$ at $25^{\circ} \mathrm{C}$

Figure 6.4: Changes in the stress response of $\alpha$ iron single crystals, loaded in [100] at $25^{\circ} \mathrm{C}$, with the set of exponential parameter variations given in Table 6.7. 

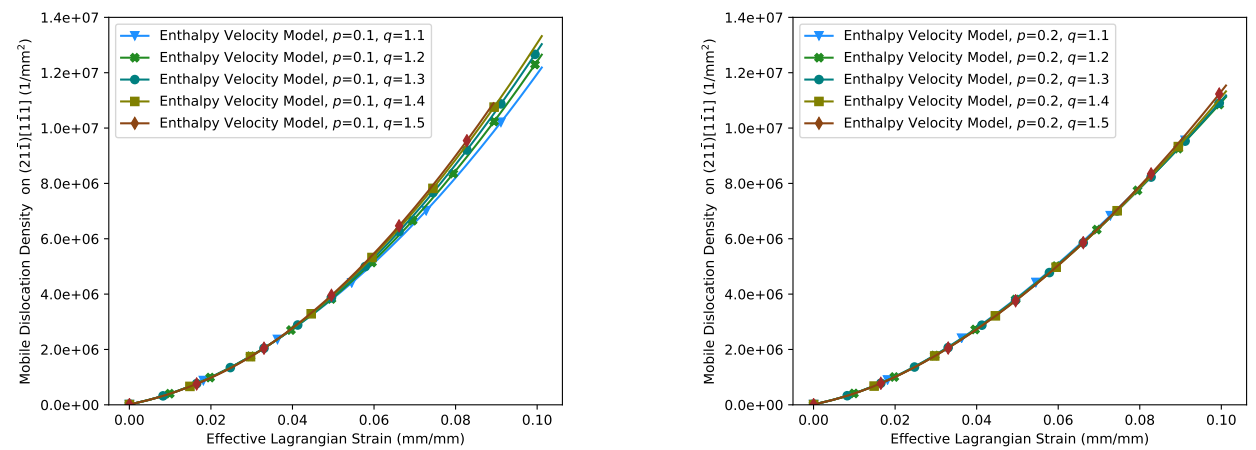

(a) Variations with $p=0.1$ at $25^{\circ} \mathrm{C}$

(b) Variations with $p=0.2$ at $25^{\circ} \mathrm{C}$
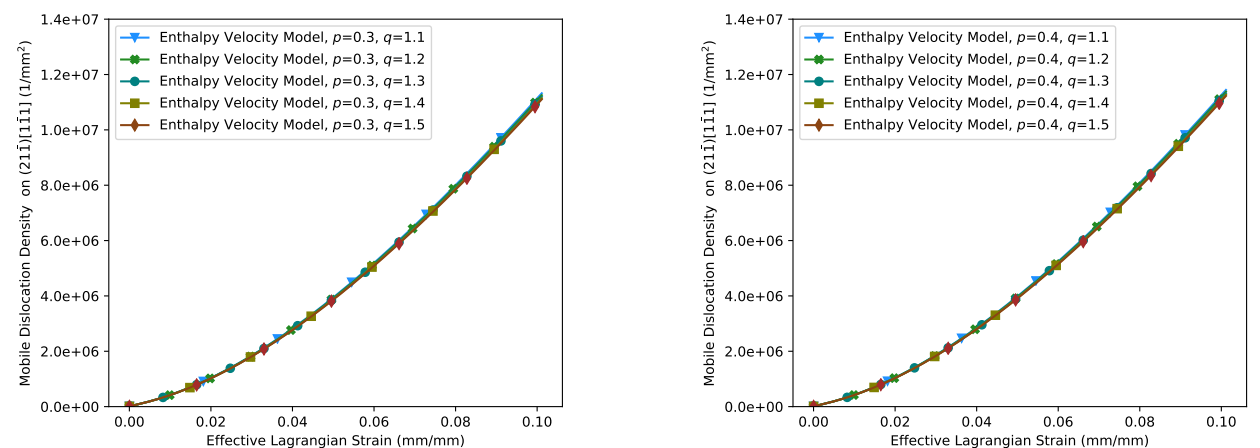

(c) Variations with $p=0.3$ at $25^{\circ} \mathrm{C}$

(d) Variations with $p=0.4$ at $25^{\circ} \mathrm{C}$

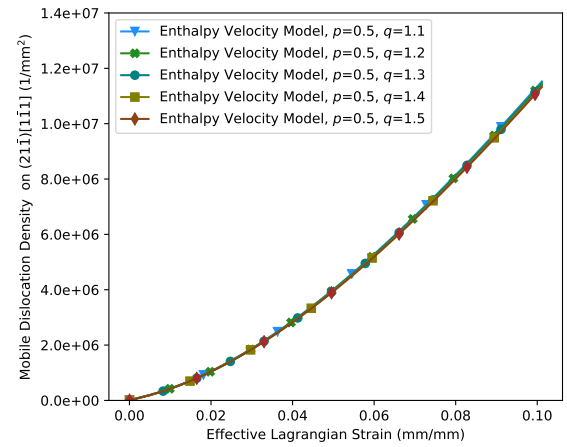

(e) Variations with $p=0.5$ at $25^{\circ} \mathrm{C}$

Figure 6.5: Evolution of the mobile dislocations on the active slip system $(21 \overline{1})[1 \overline{1} 1]$ in single crystal $\alpha$ iron, at $25^{\circ} \mathrm{C}$, with the exponential parameter sets listed in Table 6.7. 
We have separated the results of the simulations conducted at $25^{\circ} \mathrm{C}$, Table 6.7 , into Figure 6.4 and Figure 6.5 and the simulation results from $150^{\circ} \mathrm{C}$, Table 6.8 , into Figure 6.6 and Figure 6.7. In the enthalpy velocity model results we observe the same cross over behavior we observed in the temperature range study, Section 6.2.1. In this exponential constant variation study the cross over behavior is present at low $p$ values and high $q$ values. At the lower temperature this crossing of stress strain curves is demonstrated only for low values of $p$ at 0.1 and 0.2 combined with high values of $q$ at 1.4 and 1.5, Figures $6.4 \mathrm{a}$ and $6.4 \mathrm{~b}$. In the simulations set conducted at $150^{\circ} \mathrm{C}$ this cross over hardening behavior extends into the middle of the tested range of $p$ and $q$ values, Figures 6.6a, 6.6b, and 6.6c.

As in the power law velocity study, the mobile dislocation density on the representative active slip system is less sensitive to the variation in the exponential constants, Figure 6.5 and Figure 6.7. Nonetheless the cross over trend we observed in the stress response is also present in the mobile dislocation evolution for low values of $p$ and high values of $q$. The trend is noticeable in the mobile dislocation evolution at $25^{\circ} \mathrm{C}$, Figures $6.5 \mathrm{a}$ and $6.5 \mathrm{~b}$ and is accentuated in the equivalent simulations run at $150^{\circ} \mathrm{C}$, Figures $6.7 \mathrm{a}$ and $6.7 \mathrm{~b}$. These results demonstrate that the enthalpy-based velocity model may inhibit the dislocation evolution equations, depending on the velocity model exponential constant parameter values. 


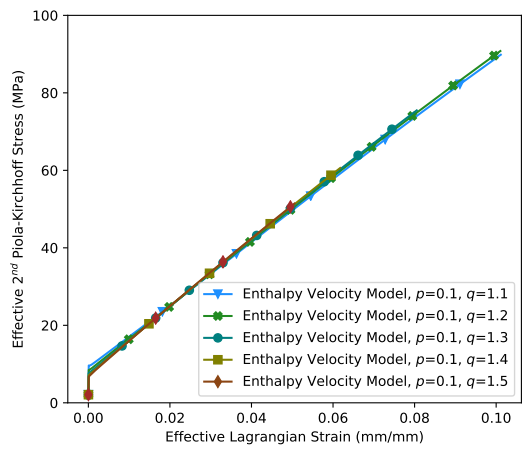

(a) Variations with $p=0.1$ at $150^{\circ}$

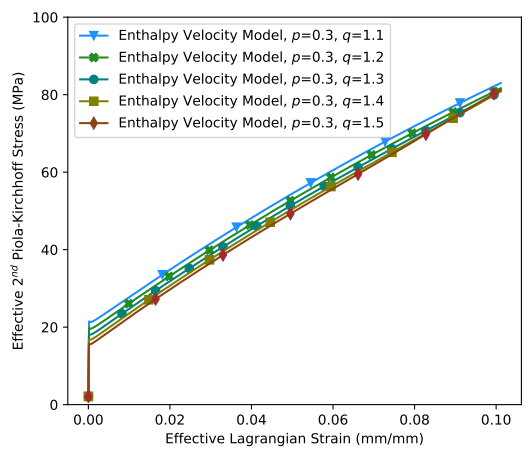

(c) Variations with $p=0.3$ at $150^{\circ}$

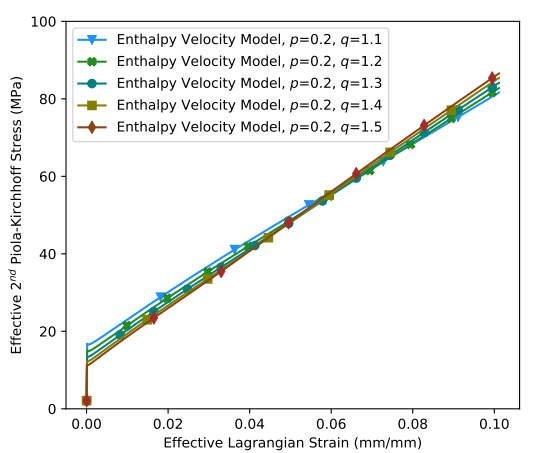

(b) Variations with $p=0.2$ at $150^{\circ}$

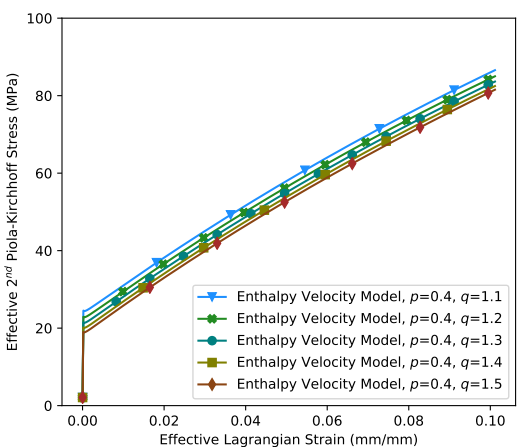

(d) Variations with $p=0.4$ at $150^{\circ}$

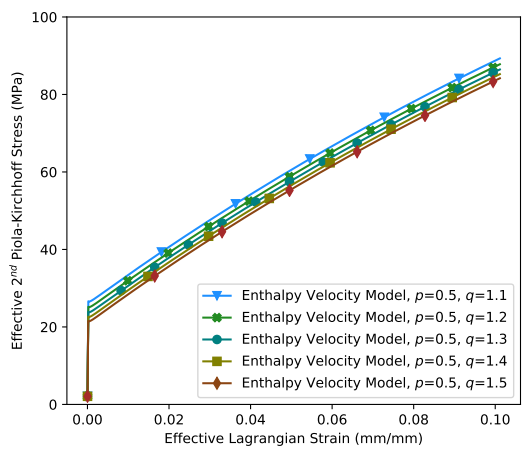

(e) Variations with $p=0.5$ at $150^{\circ}$

Figure 6.6: Changes in the stress response of $\alpha$ iron single crystals, loaded in [100] at $150^{\circ} \mathrm{C}$, with the set of exponential parameter variations given in Table 6.8. 


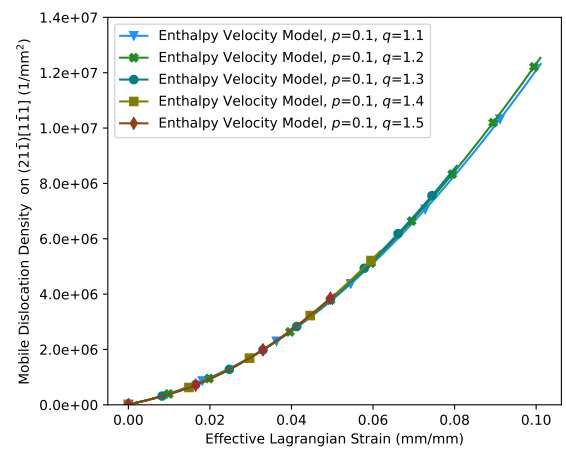

(a) Variations with $p=0.1$ at $150^{\circ}$

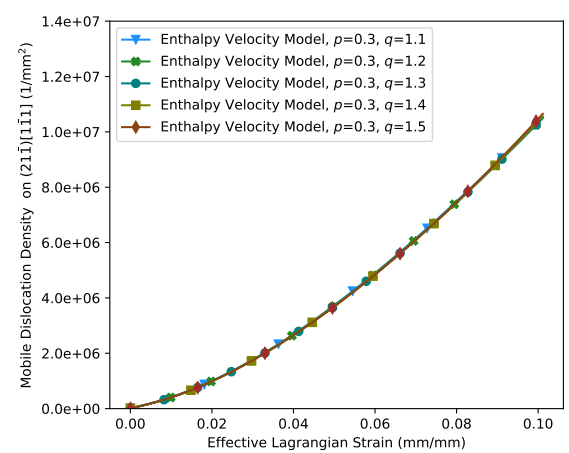

(c) Variations with $p=0.3$ at $150^{\circ}$

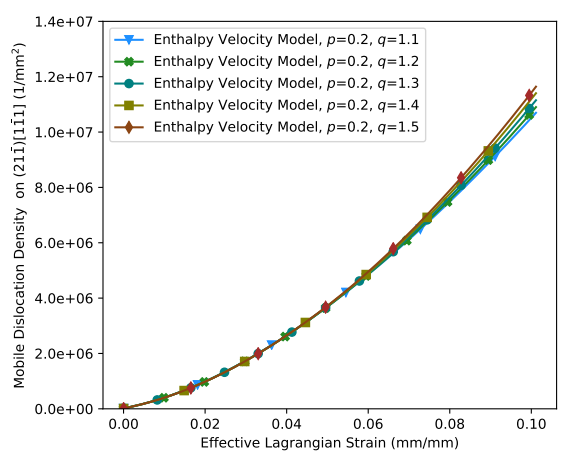

(b) Variations with $p=0.2$ at $150^{\circ}$

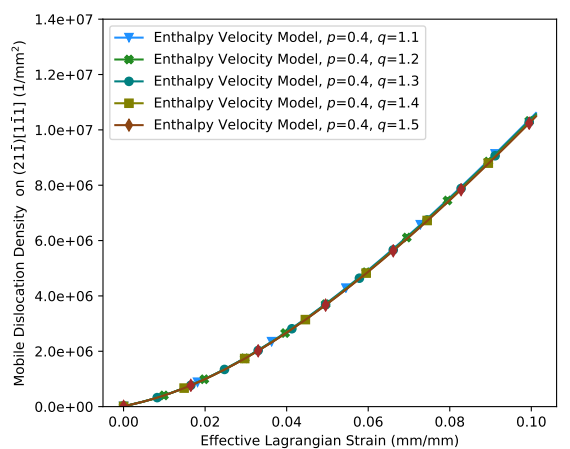

(d) Variations with $p=0.4$ at $150^{\circ}$

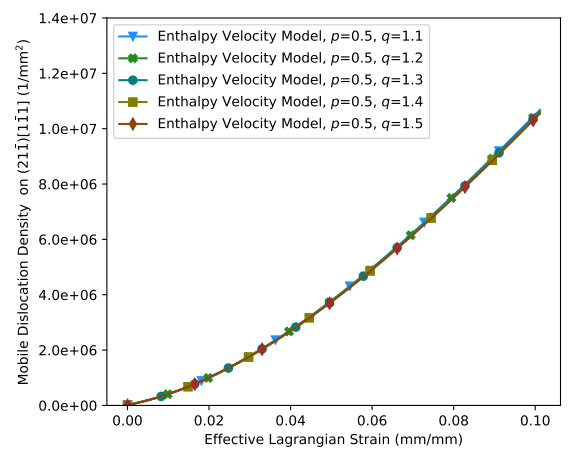

(e) Variations with $p=0.5$ at $150^{\circ}$

Figure 6.7: Evolution of the mobile dislocations on the active slip system $(21 \overline{1})[1 \overline{1} 1]$ in single crystal $\alpha$ iron, at $150^{\circ} \mathrm{C}$, with the exponential parameter sets listed in Table 6.8. 
The presence of the cross over trend in the variation of the enthalpy-based model exponential parameter study indicates the potential for inconsistent results with this glide velocity model. Poorly calibrated applications of this model may produce inconsistent and nonphysical results. The interaction of the two exponential shape function parameters introduces additional uncertainity into application beyond those we have discussed in the temperature variation study, Section 6.2.1. The power law velocity model, which does not demonstrate the potential to generate nonphysical simulation results, is therefore a more reliable dislocation glide velocity mode for crystal plasticity simulations spanning a range of temperatures.

\subsubsection{Temperature Dependent Modification to the Power Law Model}

The reliability of the power law dislocation glide velocity model motivates the consideration of additional methods to introduce a direct dependence on temperature. In a study of the isotropic thermoviscoplastic materials Zbib and Jubran proposed a temperature dependent flow rule with a homologous temperature factor [203]. Adopting this same approach we propose, for future study, the modification to the power law dislocation glide velocity, Eq (6.1), to introduce a direct temperature dependence:

$$
v_{\text {glide }}^{\alpha}=v_{o}\left|\frac{\tau^{\alpha}}{g^{\alpha}}\right|^{1 / m} \cdot\left(1-\frac{T-T_{r}}{T_{m}-T_{r}}\right)^{1 / m^{*}} \operatorname{sign}\left(\tau^{\alpha}\right), \text { if } \tau^{\alpha} \geq g_{o}^{\alpha}
$$


where $T$ is the current temperature, $T_{r}$ is a representative room temperature, $T_{m}$ is the metal melting temperature, and $m^{*}$ is the homologous temperature exponent, following [203]. This modification will provide an increased increased dislocation glide velocity with increasing temperature.

\subsection{Conclusions and Recommendations}

In this chapter we have compared two commonly used dislocation glide velocity models in their ability to capture softening in response to temperature increases and in their stability under material specific exponential parameter variations. Based on both experimental data and engineering observations, we expect that the stress-strain response of a metal will soften with exposure to increasing temperature. These expectations are in line with the concept of the DBTT curve we have discussed in Chapter 1 in which lower temperature samples deform less than samples at higher temperatures at the same stress. In order to achieve our goal of predicting the influence of irradiation microstructure evolution on the engineering scale DBTT curve, we require a crystal plasticity dislocation glide velocity model which responds consistently to changes in the temperature.

Inconsistency in the crystal plasticity simulation response to changes in temperature, such as those observed in the enthalpy-based glide velocity model, do not sat- 
isfy this requirement. The further variability introduced into the stress response of the enthalpy-based velocity equation by changes in the exponential parameters compounds the probability of a non physical prediction from this glide velocity model. Although the power law velocity model does not demonstrate a large change in the simulation response, the consistency with which this model adheres to the expected trend of softer stress responses under increasing temperature makes the power law dislocation glide velocity model a more reliable simulation choice. We suggest a straightforward future modification to the power law dislocation glide velocity model with a homologous temperature factor to introduce temperature dependence into this reliable velocity model.

We note the limitations of this study included a reliance solely on dislocation glide when additional deformation mechanisms, such as climb and creep, are know to be active at higher temperatures. Incorporating models for both of these mechanisms into the CDD crystal plasticity framework will expand the usability of the framework to higher temperatures. 


\section{CHAPTER 7. CONCLUSION}

In this work we have presented a CDD crystal plasticity framework and a set of model additions for nuclear power plant structural application simulations. The strength of this framework we have presented here is the ability to capture physical interactions among different crystalline defect populations which are observed in the microstructure of these structural materials. These crystalline defects include glide mobile and immobile dislocations, geometrically necessary dislocations, twinning dislocations, irradiation defects, and thermal aging defects as we discussed in Chapter 2. In this work we have presented the numerical implementation of models for interactions among these different dislocations and defects with benchmark verifications for these models, along with comparisons of the model predictions against experimental data.

We have introduced the MOOSE implementation of the CDD crystal plasticity framework within the context of $\mathrm{BCC} \alpha$ iron, which is used in the reactor pressure vessels of current light water reactors. In Chapter 3 we have demonstrated the ability of the CDD dislocation evolution equations to capture the orientation specific behavior of single $\alpha$ iron crystals. We have also highlighted the importance of the Monte Carlo stochastic approach to modeling cross slip, in conjunction with the anisotropic hardening of slip systems. We have concluded this chapter with a demonstration of 
the CDD crystal plasticity framework to a polycrystalline application, where the CDD simulations align well with experimental data from both unirradiated and irradiated polycrystalline $\alpha$ iron.

We have next introduced the addition of a model for geometrically necessary dislocations (GNDs) to capture the effect of plastic lattice curvature from deformation on the evolution of the glide dislocations. In the first portion of Chapter 4 we have contrasted two different methods of calculating the GNDs for mesh independence and solution smoothness before proceeding with the method based on the nodal ZZ-patch recovery approach to calculate the Nye's dislocation tensor. We have computed a total GND density value from the Nye's tensor. We then examined the predictions of the GND model addition to the CDD crystal plasticity framework within a set of single and bicrystal simulations. The results of these simulations demonstrated that our GNDs model implementation is sensitive to changes in the grain boundary angle.

Our next model addition focused on the another type of dislocation: twinning dislocations. Motivated by experimental results of micro-pillar compression testing on a FCC nickel alloy, in Chapter 5 we have discussed a twin shear increment model and demonstrated the implementation of this model as an additive component in the plastic velocity gradient. We have also introduced a hardening term for the thermally aged long range ordered precipitates into the glide dislocation slip resistance model. Nickel based alloys are used for structural materials in the steam generation 
components of current reactors and are being considered for the reactor vessel of Gen IV molten salt reactors. After demonstrating the use of the twinning model addition in a set of benchmark problems, we have applied the twinning model addition to the CDD crystal plasticity framework in simulations of the micro-pillar compression tests.

In the final chapter of this work we have presented the results of a comparative study between two dislocation glide velocity models on the basis of sensitivity to temperature and the stability of the calculated solution under variations of the material-specific parameters. We have concluded in Chapter 6 that the power law glide dislocation velocity model is better suited for CDD crystal plasticity simulations. While this glide velocity model is more simple, it provides stable results over a wide range of temperatures in which the enthalpy-based velocity model demonstrates some nonphysical results.

\subsection{Future Development and Potential Applications}

We suggest a set of future applications for the CDD crystal plasticity framework which we have implemented into MOOSE in this work. As a set of MOOSE-based code, the CDD crystal plasticity framework can be coupled to a variety of physics also implemented into the multiphysics platform of MOOSE. We outline here a few of the future developmental goals for the coupling of the crystal plasticity code to 
other MOOSE code capabilities.

Extending the polycrystalline capabilities of the CDD crystal plasticity framework, including the GND model, is one of our priorities for future development. One the numerical implementation issues of the nodal ZZ-patch recovery method, which we discussed in Chapter 4, have been addressed, we will apply the CDD framework to a set of realistic and complex microstructures. After verifying and calibrating the CDD GND model using EBSD data in a manner following [48, 36, 87], we will employ the CDD crystal plasticity framework to study the impact of microstructure resulting from advanced manufacturing techniques. We will explore coupling the CDD crystal plasticity code to the phase field capabilities within MOOSE to better inform the microstructure geometry and defect populations. This coupling approach is along the lines of other crystal plasticity and phase field coupling efforts [53]; however, the shared code base of these two MOOSE-based codes will allow for tighter code coupling, perhaps as a MultiApp.

As one developmental aspect of this future coupling between the CDD crystal plasticity and the phase field codes, we will modify the CDD codes developed as part of this work, Appendix A., to utilize the newly developed automatic differential capabilities in MOOSE. This automatic differentiation is possible through the use of the third party library MetaPhysicL and computes the stress residuals and Jacobians as a function of the temperature, displacement, and relative material properties at 
run time. This capability produces more accurate Jacobians and thus better solution convergence.

Beyond the structural materials we have studied in this work, dislocation and defect populations play a large role in the performance of nuclear fuels. Within light water reactor research our focus is on $\mathrm{UO}_{2}$ fuel pellets. These fuel pellets undergo significant twinning [207]. The twinning model which we have implemented in Chapter 5 could be extended to allow for large twinning through an expansion of the orientation used to calculate the stress response. An expansion of the constitutive twinning model to track densities of twin dislocations would enable the study of deformation mechanisms in $\mathrm{UO}_{2}$ fuel with crystal plasticity.

Outside of the MOOSE platform, we will investigate coupling the CDD crystal plasticity framework code to a standalone code, SCIANTIX, that predicts fission gas bubble formation and release in $\mathrm{UO}_{2}$ and MOX fuels [149]. Fission gas release from within the fuel pellet depends heavily on the microstructure, where gas atoms travel along dislocations before accessing the grain boundary network to escape the fuel pellets [150]. Dislocations are also known to promote the formation of larger fission gas bubbles within the ceramic fuel by trapping and entangling these bubbles, and the dislocation cores act as a source of vacancies for the bubbles [16]. Enhancing the SCIANTIX code with models from the CDD crystal plasticity framework will allow us to study how the deforming microstructure affects fission gas release. 
Finally we propose the investigation of forming a reduced order model based on the physical interaction mechanisms modeled in the CDD framework. A reduced order model could compute engineering scale material property behavior based on mesoscale structure as determined by the CDD model in a more computationally effective manner. The limitations on material length scale and simulation time scales are challenges with the mesoscale modeling field has attempted to address with a variety of approaches, including reduced order models and DFT simulations [80, 97]. The challenge in this future development area is to retain the influence of mesoscale features within the microstructure, such as grain boundaries, without sacrificing computational speed.

These future applications leverage the physical basis on which the CDD crystal plasticity framework was developed. This physical foundation allows these future developments to investigate the impact of microstructure evolution on engineering scale properties in a wide range of operating and manufacturing conditions. 


\section{APPENDIX}

\section{A. CDD Crystal Plasticity Classes Implemented in MOOSE}

The following classes were developed within the MOOSE code tensor mechanics module to complete the simulations shown in this work:

- ComputeCrystalPlasticityStress

- ComputeElasticityTensorConstantRotationCP

- CrystalPlasticityUpdate

- CrystalPlasticityCDDUpdateBase

- CrystalPlasticityKalidindiUpdate

- CrystalPlasticityCDDBCCFeUpdate

- CrystalPlasticityCDDEnthalpyVelocityBCCFeUpdate

- CrystalPlasticityCDDEnthalpyVelocityUpdateBase

- CrystalPlasticityCDDNiAlloyUpdate

- CrystalPlasticityCDDNiAlloyUpdateInternalFcnsGNDs

- CrystalPlasticityCDDUpdateBaseInternalFcnsGNDs 


\section{BIBLIOGRAPHY}

[1] Rashid K Abu Al-Rub, George Z Voyiadjis, and Douglas J Bammann. A thermodynamic based higher-order gradient theory for size dependent plasticity. International Journal of Solids and Structures, 44(9):2888-2923, 2007.

[2] Elias C Aifantis. On the gradient approach-relation to eringens nonlocal theory. International Journal of Engineering Science, 49(12):1367-1377, 2011.

[3] S Akarapu, HM Zbib, and DF Bahr. Analysis of heterogeneous deformation and dislocation dynamics in single crystal micropillars under compression. International Journal of Plasticity, 26(2):239-257, 2010.

[4] Alankar Alankar, David P Field, and Dierk Raabe. Plastic anisotropy of electro-deposited pure $\alpha$-iron with sharp crystallographic $<111>$ texture in normal direction: Analysis by an explicitly dislocation-based crystal plasticity model. International Journal of Plasticity, 52:18-32, 2014.

[5] Sebastien Allain, J. P. Chateau, and O. Bouaziz. A physical model of the twinning-induced plasticity effect in a high manganese austenitic steel. Materials Science and Engineering A, 387-389(1-2 SPEC. ISS.):143-147, 2004.

[6] T. R. Allen and J. T. Busby. Radiation damage concerns for extended light water reactor service. JOM, 61(7):29-34, 2009. 
[7] A Arsenlis and DM Parks. Crystallographic aspects of geometrically-necessary and statistically-stored dislocation density. Acta Materialia, 47(5):1597-1611, 1999.

[8] A. Arsenlis, M. Rhee, G. Hommes, R. Cook, and J. Marian. A dislocation dynamics study of the transition from homogeneous to heterogeneous deformation in irradiated body-centered cubic iron. Acta Materialia, 60(9):3748 $3757,2012$.

[9] A. Arsenlis, B. D. Wirth, and M. Rhee. Dislocation density-based constitutive model for the mechanical behaviour of irradiated Cu. Philosophical Magazine, 84(34):3617-3635, 2004.

[10] Athanasios Arsenlis and David M. Parks. Modeling the evolution of crystallographic dislocation density in crystal plasticity. Journal of the Mechanics and Physics of Solids, 50(9):1979-2009, 2002.

[11] Robert J Asaro. Crystal plasticity. Journal of Applied Mechanics, 50(4b):921934, 1983.

[12] M F Ashby. The deformation of plastically non-homogeneous materials. The Philosophical Magazine: A Journal of Theoretical Experimental and Applied Physics, 21(170):399-424, 1970. 
[13] Harm Askes and Elias C Aifantis. Gradient elasticity in statics and dynamics: an overview of formulations, length scale identification procedures, finite element implementations and new results. International Journal of Solids and Structures, 48(13):1962-1990, 2011.

[14] DJ Bacon, F Gao, and Yu N Osetsky. The primary damage state in fcc, bcc and hcp metals as seen in molecular dynamics simulations. Journal of Nuclear Materials, 276(1-3):1-12, 2000.

[15] JE Bailey and PB Hirsch. The dislocation distribution, flow stress, and stored energy in cold-worked polycrystalline silver. Philosophical Magazine, 5(53):485-497, 1960.

[16] T. Barani, A. Magni, D. Pizzocri, L. Cognini, P. Van Uffelen, L. Luzzi, and G. Pastore. Modeling and assessment of intra-grannular bubble evolution and coarsening in uranium dioxide. In NuMat, Seattle, Washington, 2018.

[17] Nathan R Barton, Athanasios Arsenlis, and Jaime Marian. A polycrystal plasticity model of strain localization in irradiated iron. Journal of the Mechanics and Physics of Solids, 61(2):341-351, 2013.

[18] I. J. Beyerlein and C. N. Tomé. A dislocation-based constitutive law for pure Zr including temperature effects. International Journal of Plasticity, 24(5):867895, 2008. 
[19] Irene J. Beyerlein, Nathan A. Mara, Dhriti Bhattacharyya, David J. Alexander, and Carl T. Necker. Texture evolution via combined slip and deformation twinning in rolled silver-copper cast eutectic nanocomposite. International Journal of Plasticity, 27(1):121-146, 2011.

[20] Jérôme Blaizot, Thibaut Chaise, Daniel Nélias, Michel Perez, Sophie Cazottes, and Philippe Chaudet. Constitutive model for nickel alloy 690 (inconel 690) at various strain rates and temperatures. International Journal of Plasticity, 80:139-153, 2016.

[21] Arthur P. Boresi and Richard J. Schmidt. Advanced Mechanics of Materials. John Wiley \& Sons, 6 edition, 2003.

[22] Allen F. Bower. Applied Mechanics of Solids. Taylor \& Francis, 2010.

[23] D Buckthorpe. Introduction to generation iv nuclear reactors. In Structural Materials for Generation IV Nuclear Reactors, pages 1-22. Elsevier, 2017.

[24] E. P. Busso, F. T. Meissonnier, and N. P. O’Dowd. Gradient-dependent deformation of two-phase single crystals. Journal of the Mechanics and Physics of Solids, 48(11):2333-2361, 2000.

[25] François Cattant, Didier Crusset, and Damien Féron. Corrosion issues in nuclear industry today. Materials Today, 11(10):32-37, 2008. 
[26] Augustin-Louis Cauchy. Sur la condensation et la dilatation des corps solides. Exercices de Mathématiques, 2(1827):60-69, 1827. in French.

[27] Paolo Cermelli and Morton E Gurtin. On the characterization of geometrically necessary dislocations in finite plasticity. Journal of the Mechanics and Physics of Solids, 49(7):1539-1568, 2001.

[28] Pritam Chakraborty and S Bulent Biner. Crystal plasticity modeling of irradiation effects on flow stress in pure-iron and iron-copper alloys. Mechanics of Materials, 101:71-80, 2016.

[29] Pritam Chakraborty, Suleyman B. Biner, Yongfeng Zhang, and Benjamin W. Spencer. Crystal plasticity model of reactor pressure vessel embrittlement in Grizzly. Technical report, Idaho National Labratory (INL), Idaho Falls, ID (United States), 2015.

[30] KS Chan, Y-D Lee, and Y-M Pan. First-principles computations of mechanical properties of ni $2 \mathrm{cr}$ and ni 2 mo. Metallurgical and Materials Transactions A, 37(3):523-537, 2006.

[31] Long-Qing Chen. Phase-field models for microstructure evolution. Annual review of materials research, 32(1):113-140, 2002.

[32] Ke-Shen Cheong and Esteban P Busso. Discrete dislocation density modelling 
of single phase FCC polycrystal aggregates. Acta Materialia, 52(19):5665$5675,2004$.

[33] Eugene Cosserat, François Cosserat, et al. Théorie des corps déformables. A. Hermann et fils,, 1909. In French.

[34] A Cruzado, B Gan, M Jiménez, D Barba, K Ostolaza, A Linaza, JM MolinaAldareguia, J Llorca, and J Segurado. Multiscale modeling of the mechanical behavior of in718 superalloy based on micropillar compression and computational homogenization. Acta Materialia, 98:242-253, 2015.

[35] Hong Dai and David M Parks. Geometrically-necessary dislocation density and scaledependent crystal plasticity. In A.S. Khan, editor, Proceedings of Plasticity, pages 17-18. Neat Press, 1997.

[36] Suchandrima Das, Felix Hofmann, and Edmund Tarleton. Consistent determination of geometrically necessary dislocation density from simulations and experiments. International Journal of Plasticity, 109:18-42, 2018.

[37] TD de la Rubia, HM Zbib, TA Khraishi, BD Wirth, M Victoria, and MJ Caturla. Multiscale modelling of plastic flow localization in irradiated materials. Nature, 406(6798):871-4, 2000. 
[38] DM Dimiduk, MD Uchic, and TA Parthasarathy. Size-affected single-slip behavior of pure nickel microcrystals. Acta Materialia, 53(15):4065-4077, 2005.

[39] Véronique Doquet. Twinning and multiaxial cyclic plasticity of a low stackingfault-energy fcc alloy. Acta Metallurgica et Materialia, 41(8):2451-2459, 1993.

[40] James J Duderstadt. Nuclear Reactor Analysis. Wiley, 1976.

[41] F. P.E. Dunne, R. Kiwanuka, and A. J. Wilkinson. Crystal plasticity analysis of micro-deformation, lattice rotation and geometrically necessary dislocation density. Proceedings of the Royal Society A: Mathematical, Physical and Engineering Sciences, 468(2145):2509-2531, 2012.

[42] Fionn Dunne and Nik Petrinic. Introduction to Computational Plasticity. Oxford University Press on Demand, 2005.

[43] Jaafar A El-Awady. Unravelling the physics of size-dependent dislocationmediated plasticity. Nature communications, 6:5926, 2015.

[44] BS El-Dasher, BL Adams, and AD Rollett. Experimental recovery of geometrically necessary dislocation density in polycrystals. Scripta Materialia, 48(2):141-145, 2003.

[45] L. P. Evers, W. A.M. Brekelmans, and M. G.D. Geers. Non-local crystal 
plasticity model with intrinsic SSD and GND effects. Journal of the Mechanics and Physics of Solids, 52(10):2379-2401, 2004.

[46] L. P. Evers, D. M. Parks, W. A.M. Brekelmans, and M. G.D. Geers. Crystal plasticity model with enhanced hardening by geometrically necessary dislocation accumulation. Journal of the Mechanics and Physics of Solids, 50(11):2403-2424, 2002.

[47] Huiyang Fei, Amit Abraham, Nikhilesh Chawla, and Hanqing Jiang. Evaluation of micro-pillar compression tests for accurate determination of elasticplastic constitutive relations. Journal of Applied Mechanics, 79(6):061011, 2012.

[48] D. P. Field, C. C. Merriman, N. Allain-Bonasso, and F. Wagner. Quantification of dislocation structure heterogeneity in deformed polycrystals by EBSD. Modelling and Simulation in Materials Science and Engineering, 20(2), 2012.

[49] P Fischer, J Mergheim, and P Steinmann. On the $\mathrm{C}^{1}$ continuous discretization of non-linear gradient elasticity: A comparison of nem and fem based on bernstein-bézier patches. International Journal for Numerical Methods in Engineering, 82(10):1282-1307, 2010.

[50] N.A. Fleck and J. W. Hutchinson. Strain Gradient Plasticity. Advances in Applied Mechanics, 33:296-361, 1997. 
[51] NA Fleck and JW Hutchinson. A reformulation of strain gradient plasticity. Journal of the Mechanics and Physics of Solids, 49(10):2245-2271, 2001.

[52] S Forest, G Cailletaud, and R Sievert. A cosserat theory for elastoviscoplastic single crystals at finite deformation. Arch. Mech, 49:705-736, 1997.

[53] Marianne M Francois, Amy Sun, Wayne E King, Neil Jon Henson, Damien Tourret, Ccut Allan Bronkhorst, Neil N Carlson, Christopher Kyle Newman, Terry Scot Haut, Jozsef Bakosi, et al. Modeling of additive manufacturing processes for metals: Challenges and opportunities. Current Opinion in Solid State and Materials Science, 21, 2017.

[54] F Gao, DJ Bacon, Yu N Osetsky, PEJ Flewitt, and TA Lewis. Properties and evolution of sessile interstitial clusters produced by displacement cascades in $\alpha$-iron. Journal of Nuclear Materials, 276(1-3):213-220, 2000.

[55] H Gao and Y Huang. ScienceDirect.com - Scripta Materialia - Geometrically necessary dislocation and size-dependent plasticity. Scripta Mater, 48:113-118, 2003.

[56] H. Gao, Y. Huang, W. D. Nix, and J. W. Hutchinson. Mechanism-based strain gradient plasticity - I. Theory. Journal of the Mechanics and Physics of Solids, 47(6):1239-1263, 1999. 
[57] Derek Gaston, Chris Newman, Glen Hansen, and Damien Lebrun-Grandie. Moose: A parallel computational framework for coupled systems of nonlinear equations. Nuclear Engineering and Design, 239(10):1768-1778, 2009.

[58] George Green. On the propagation of light in crystallized media. Transactions of the Cambridge Philosophical Society, 7:437-444, 1841.

[59] Peter Gudmundson. A unified treatment of strain gradient plasticity. Journal of the Mechanics and Physics of Solids, 52(6):1379-1406, 2004.

[60] Morton E Gurtin. A gradient theory of single-crystal viscoplasticity that accounts for geometrically necessary dislocations. Journal of the Mechanics and Physics of Solids, 50(1):5-32, 2002.

[61] B. Gwalani, T. Alam, C. Miller, T. Rojhirunsakool, Y. S. Kim, S. S. Kim, M. J. Kaufman, Yang Ren, and R. Banerjee. Experimental investigation of the ordering pathway in a Ni-33 at.\%Cr alloy. Acta Materialia, 115:372-384, 2016.

[62] EO Hall. The deformation and ageing of mild steel: Iii discussion of results. Proceedings of the Physical Society, Section B, 64(9):747, 1951.

[63] K. Hazeli, H. Askari, J. Cuadra, F. Streller, R. W. Carpick, H. M. Zbib, and 
A. Kontsos. Microstructure-sensitive investigation of magnesium alloy fatigue. International Journal of Plasticity, 68:55-76, 2015.

[64] Richard W. Hertzberg. Deformation and Fracture Mechanics of Engineering Materials. John Wiley \& Sons, 4 edition, 1996.

[65] John P Hirth and Jens Lothe. Theory of Dislocations. John Wiley \& Sons, 1982.

[66] E.C. Aifantis H.M. Zbib. On the gradient-dependent theory of plasticity and shear banding. Acta Mechanica, 92:209-225, 1992.

[67] R.I. Hosford, W.F. Fleischer and W.A. Backofen. Tensile Deformation of Aluminum Single Crystals at Low Temperatures. Acta Metallurgica, 8(45):39, 1960.

[68] D.J. Hughes, D A Hansen, N. Bammann. Geometrically necessary boundaries, incidental dislocation boundaries and geometrically necessary dislocations. Scripta Materialia, 48:147-153, 2003.

[69] Derek Hull and David J Bacon. Introduction to Dislocations. Elsevier, 5 edition, 2011.

[70] Daniel E Hurtado and Michael Ortiz. Surface effects and the size-dependent 
hardening and strengthening of nickel micropillars. Journal of the Mechanics and Physics of Solids, 60(8):1432-1446, 2012.

[71] Intergovernmental Panel on Climate Change. Special report of global warming of $1.5^{\circ} \mathrm{c}$. Technical report, United Nations, 2018.

[72] International Atomic Energy Agency. Climate change \& nuclear energy. Technical report, International Atomic Energy Agency, 2018.

[73] We G Johnston and Jo J Gilman. Dislocation velocities, dislocation densities, and plastic flow in lithium fluoride crystals. Journal of Applied Physics, 30(2):129-144, 1959.

[74] Jae-Ho Jung, Young-Sang Na, Kyung-Mox Cho, Dennis M Dimiduk, and Yoon Suk Choi. Microcompression behaviors of single crystals simulated by crystal plasticity finite element method. Metallurgical and Materials Transactions A, 46(11):4834-4840, 2015.

[75] $\mathrm{R}$ Kalidindi. Incorporation of Deformation Twinning in Models. J.Mech.Phys.Solids, 46(2):267-290, 1998.

[76] Surya R Kalidindi. Modeling anisotropic strain hardening and deformation textures in low stacking fault energy fcc metals. International Journal of Plasticity, 17(6):837-860, 2001. 
[77] S. Karthikeyan, R. R. Unocic, P. M. Sarosi, G. B. Viswanathan, D. D. Whitis, and M. J. Mills. Modeling microtwinning during creep in Ni-based superalloys. Scripta Materialia, 54(6):1157-1162, 2006.

[78] AS Keh. Work hardening and deformation sub-structure in iron single crystals deformed in tension at 298 K. Philosophical Magazine, 12(115):9-30, 1965.

[79] Shahriyar Keshavarz and Somnath Ghosh. Multi-scale crystal plasticity finite element model approach to modeling nickel-based superalloys. Acta Materialia, 61(17):6549-6561, 2013.

[80] Shahriyar Keshavarz and Somnath Ghosh. Hierarchical crystal plasticity fe model for nickel-based superalloys: Sub-grain microstructures to polycrystalline aggregates. International Journal of Solids and Structures, 55:17-31, 2015.

[81] Shahriyar Keshavarz, Somnath Ghosh, Andrew CE Reid, and Stephen A Langer. A non-schmid crystal plasticity finite element approach to multi-scale modeling of nickel-based superalloys. Acta Materialia, 114:106-115, 2016.

[82] Akhtar S Khan and Sujian Huang. Continuum Theory of Plasticity. John Wiley \& Sons, 1995.

[83] Daniel Kiener, PJ Guruprasad, Shyam M Keralavarma, Gerhard Dehm, and 
Ahmed Amine Benzerga. Work hardening in micropillar compression: In situ experiments and modeling. Acta Materialia, 59(10):3825-3840, 2011.

[84] Seong Gyoon Kim, Won Tae Kim, and Toshio Suzuki. Phase-field model for binary alloys. Physical review e, 60(6):7186, 1999.

[85] UF Kocks. Laws for work-hardening and low-temperature creep. Journal of Engineering Materials and Technology, 98(1):76-85, 1976.

[86] M Kolbe. The high temperature decrease of the critical resolved shear stress in nickel-base superalloys. Materials Science and Engineering A, 319:383-387, 2001.

[87] Peter Joachim Konijnenberg, Stefan Zaefferer, and Dierk Raabe. Assessment of geometrically necessary dislocation levels derived by 3d ebsd. Acta Materialia, 99:402-414, 2015.

[88] M. Kothari and L. Anand. Elasto-viscoplastic constitutive equations for polycrystalline metals: Application to tantalum. Journal of the Mechanics and Physics of Solids, 46(1):51-83, 1998.

[89] RW Kozar, A Suzuki, WW Milligan, JJ Schirra, MF Savage, and TM Pollock. Strengthening mechanisms in polycrystalline multimodal nickel-base superalloys. Metallurgical and Materials Transactions A, 40(7):1588-1603, 2009. 
[90] E Kröner and A Seeger. Nicht-Lineare Elastizitatstheorie der Versetzungen und Eigenspannungen. Archive for Rational Mechanics and Analysis, 3(2):97119, 1959. In German.

[91] Ekkehart Kröner. General Continuum Theory of Dislocations And Proper Stresses, 1960.

[92] Mitsutoshi Kuroda. Higher-order gradient effects in micropillar compression. Acta Materialia, 61(7):2283-2297, 2013.

[93] Mitsutoshi Kuroda and Viggo Tvergaard. On the formulations of higher-order strain gradient crystal plasticity models. Journal of the Mechanics and Physics of Solids, 56(4):1591-1608, 2008.

[94] C.Rey L. Vincent, M. Libert, B. Marini. Towards a modelling of RPV steel brittle fracture using crystal plasticity computation on polycrystalline aggregates. Journal of Nuclear Materials, 406:91-96, 2010.

[95] Marlies Lambrecht, Lorenzo Malerba, and Abderrahim Almazouzi. Influence of different chemical elements on irradiation-induced hardening embrittlement of RPV steels. Journal of Nuclear Materials, 378(3):282-290, 2008.

[96] Marlies Lambrecht, Estelle Meslin, Lorenzo Malerba, Mercedes HernándezMayoral, Frank Bergner, Philippe Pareige, Bertrand Radiguet, and Abder- 
rahim Almazouzi. On the correlation between irradiation-induced microstructural features and the hardening of reactor pressure vessel steels. Journal of Nuclear Materials, 406(1):84-89, 2010.

[97] Ricardo A Lebensohn, Juan P Escobedo, Ellen K Cerreta, Darcie DennisKoller, Curt A Bronkhorst, and John F Bingert. Modeling void growth in polycrystalline materials. Acta Materialia, 61(18):6918-6932, 2013.

[98] Ricardo A Lebensohn and CN Tomé. A self-consistent anisotropic approach for the simulation of plastic deformation and texture development of polycrystals: application to zirconium alloys. Acta metallurgica et materialia, 41(9):26112624, 1993.

[99] MG Lee, H Lim, BL Adams, JP Hirth, and RH Wagoner. A dislocation densitybased single crystal constitutive equation. International Journal of Plasticity, 26(7):925-938, 2010.

[100] Seok-Woo Lee and William D Nix. Geometrical analysis of 3d dislocation dynamics simulations of fcc micro-pillar plasticity. Materials Science and Engineering: A, 527(7-8):1903-1910, 2010.

[101] Dongsheng Li, Hussein Zbib, Xin Sun, and Mohammad Khaleel. Predicting plastic flow and irradiation hardening of iron single crystal with mechanism- 
based continuum dislocation dynamics. International Journal of Plasticity, $52: 3-17,2014$

[102] H. Liang and F. P E Dunne. GND accumulation in bi-crystal deformation: Crystal plasticity analysis and comparison with experiments. International Journal of Mechanical Sciences, 51(4):326-333, 2009.

[103] H Lim, MG Lee, JH Kim, BL Adams, and RH Wagoner. Simulation of polycrystal deformation with grain and grain boundary effects. International Journal of Plasticity, 27(9):1328-1354, 2011.

[104] Xianwu Ling, MF Horstemeyer, and GP Potirniche. On the numerical implementation of $3 \mathrm{~d}$ rate-dependent single crystal plasticity formulations. International Journal for Numerical Methods in Engineering, 63(4):548-568, 2005.

[105] Qiang Liu, Anish Roy, and Vadim V Silberschmidt. Size-dependent crystal plasticity: From micro-pillar compression to bending. Mechanics of Materials, 100:31-40, 2016.

[106] Hao Lyu, Nasrin Taheri-Nassaj, and Hussein M. Zbib. A multiscale gradient-dependent plasticity model for size effects. Philosophical Magazine, 96(18):1883-1908, 2016.

[107] A Ma and F Roters. A constitutive model for fcc single crystals based on 
dislocation densities and its application to uniaxial compression of aluminium single crystals. Acta Materialia, 52(12):3603-3612, 2004.

[108] A. Ma, F. Roters, and D. Raabe. A dislocation density based constitutive model for crystal plasticity FEM including geometrically necessary dislocations. Acta Materialia, 54(8):2169-2179, 2006.

[109] A. Ma, F. Roters, and D. Raabe. On the consideration of interactions between dislocations and grain boundaries in crystal plasticity finite element modeling - Theory, experiments, and simulations. Acta Materialia, 54(8):2181-2194, 2006.

[110] S Mahajan. Critique of mechanisms of formation of deformation, annealing and growth twins: Face-centered cubic metals and alloys. Scripta Materialia, 68(2):95-99, 2013.

[111] Lawrence E Malvern. Introduction to the Mechanics of a Continuous Medium. Prentice Hall, 1969.

[112] A Marucco. Phase transformations during long-term ageing of Ni-Fe-Cr alloys in the temperature range $450-600 \${ }^{\wedge}$ o $\$ C$. Materials Science and Engineering A, 194(94):225-233, 1995. 
[113] Alessandra Marucco and Birendra Nath. Effects of ordering on the properties of Ni-Cr alloys. Journal of Materials Science, 23(6):2107-2114, 1988.

[114] IN Mastorakos and HM Zbib. A multiscale approach to study the effect of chromium and nickel concentration in the hardening of iron alloys. Journal of Nuclear Materials, 449(1):101-110, 2014.

[115] J. R. Mayeur and D. L. McDowell. A comparison of Gurtin type and micropolar theories of generalized single crystal plasticity. International Journal of Plasticity, 57:29-51, 2014.

[116] David L. McDowell. A perspective on trends in multiscale plasticity. International Journal of Plasticity, 26(9):1280-1309, 2010.

[117] H Mecking and UF Kocks. A mechanism for static and dynamic recovery. In Strength of Metals and Alloys, pages 345-350. Elsevier, 1979.

[118] F T Meissonnier, E P Busso, and N P O'Dowd. Finite element implementation of a generalised non-local rate-dependent crystallographic formulation for finite strains. International Journal of Plasticity, 17(4):601-640, 2001.

[119] Estelle Meslin, Marlies Lambrecht, Mercedes Hernández-Mayoral, Frank Bergner, Lorenzo Malerba, Philippe Pareige, Bertrand Radiguet, Alain Barbu, D Gómez-Briceño, Andreas Ulbricht, et al. Characterization of neutron- 
irradiated ferritic model alloys and a rpv steel from combined APT, SANS, TEM and PAS analyses. Journal of Nuclear Materials, 406(1):73-83, 2010.

[120] Raymond David Mindlin. Micro-structure in linear elasticity. Archive for Rational Mechanics and Analysis, 16(1):51-78, 1964.

[121] MIT Energy Initiative. The future of nuclear energy in a carbon-constrained world. Technical report, Massachusetts Institute of Technology, 2018.

[122] Tyler E Moss, Catherine M Brown, and George A Young. The Effect of Long Range Order on SCC and LTCP of a Nickel-30.7Chromium Binary Alloy. In Proceedings of the 18th International Conference on Environmental Degradation of Materials in Nuclear Power Systems Water Reactors, pages 261-279. Minerals, Metals \& Materials Society, 2018.

[123] Roman Mouginot, Teemu Sarikka, Mikko Heikkilä, Mykola Ivanchenko, Ulla Ehrnstén, Young Suk Kim, Sung Soo Kim, and Hannu Hänninen. Thermal ageing and short-range ordering of Alloy 690 between 350 and 550 C. Journal of Nuclear Materials, 485:56-66, 2017.

[124] Haël Mughrabi. On the current understanding of strain gradient plasticity. Materials Science and Engineering A, 387-389(1-2):209-213, 2004.

[125] KL Murty and I Charit. Structural materials for gen-iv nuclear reactors: 
Challenges and opportunities. Journal of Nuclear Materials, 383(1-2):189-195, 2008 .

[126] United Nations. Paris Agreement. https://unfccc.int/process-andmeetings/the-paris-agreement/the-paris-agreement, December 2015. UNTC XXVII 7.d.

[127] William D Nix and Seok-Woo Lee. Micro-pillar plasticity controlled by dislocation nucleation at surfaces. Philosophical Magazine, 91(7-9):1084-1096, 2011.

[128] J.F. Nye. Some geometrical relations in dislocated crystals. Acta Metallurgica, 1(2):153-162, 1953.

[129] George Robert Odette and Randy K Nanstad. Predictive reactor pressure vessel steel irradiation embrittlement models: issues and opportunities. JOM, 61(7):17-23, 2009.

[130] GR Odette and D Frey. Development of mechanical property correlation methodology for fusion environments. Journal of Nuclear Materials, 85:817$822,1979$.

[131] GR Odette, BD Wirth, DJ Bacon, and NM Ghoniem. Multiscale-multiphysics 
modeling of radiation-damaged materials: embrittlement of pressure-vessel steels. MRS Bulletin, 26(3):176-181, 2001.

[132] U.S Department of Energy Office of Science. Basic Research needs for Advanced Nulcear Energy Systems. Technical report, US Department of Energy Ofiice of Science, 2006.

[133] Office of Nuclear Energy. 5 fast facts about nuclear energy. https://www.energy.gov/ne/articles/5-fast-facts-about-nuclear-energy, January 2018.

[134] Office of Nuclear Energy. Nuclear power summary. Technical report, United States Department of Energy, August 2018.

[135] Tetsuya Ohashi. Numerical modelling of plastic multislip in metal crystals of FCC type. Philosophical Magazine A, 70(5):793-803, 1994.

[136] Tetsuya Ohashi. Finite-element analysis of plastic slip and evolution of geometrically necessary dislocations in fcc crystals. Philosophical Magazine Letters, 75(2):51-58, 1997.

[137] Tetsuya Ohashi. A new model of scale dependent crystal plasticity analysis. In IUTAM Symposium on Mesoscopic Dynamics of Fracture Process and Materials Strength, pages 97-106. Springer, 2004. 
[138] Tetsuya Ohashi, Masato Kawamukai, and Hussein Zbib. A multiscale approach for modeling scale-dependent yield stress in polycrystalline metals. International Journal of Plasticity, 23(5):897-914, 2007.

[139] Donald R Olander. Fundamental aspects of nuclear reactor fuel elements. Technical report, California Univ., Berkeley (USA). Dept. of Nuclear Engineering, 1976.

[140] David L Olmsted, Kedar Y Hardikar, and Rob Phillips. Lattice resistance and peierls stress in finite size atomistic dislocation simulations. Modelling and Simulation in Materials Science and Engineering, 9(3):215, 2001.

[141] E Orowan. Zur Kristallplastizität. III. Zeitschrift für Physik, 89(9-10):634-659, 1934. in German.

[142] E Orowan. Problems of plastic gliding. Proceedings of the Physical Society, $52(1): 8,1940$.

[143] Yu N Osetsky, DJ Bacon, A Serra, BN Singh, and SI Golubov. Stability and mobility of defect clusters and dislocation loops in metals. Journal of Nuclear Materials, 276(1-3):65-77, 2000.

[144] Anirban Patra and David L. McDowell. Crystal plasticity-based constitutive 
modelling of irradiated bcc structures. Philosophical Magazine, 92(7):861-887, 2012 .

[145] Anirban Patra and David L McDowell. Continuum modeling of localized deformation in irradiated BCC materials. Journal of Nuclear Materials, 432(1):414427, 2013.

[146] D Peirce, RJ Asaro, and A Needleman. An analysis of nonuniform and localized deformation in ductile single crystals. Acta metallurgica, 30(6):1087-1119, 1982.

[147] Cody J. Permann. Private Communication, 2018.

[148] NJ Petch. The cleavage strength of polycrystals. Journal of the Iron and Steel Institute, 174:25-28, 1953.

[149] D Pizzocri, G Pastore, T Barani, A Magni, L Luzzi, P Van Uffelen, SA Pitts, A Alfonsi, and JD Hales. A model describing intra-granular fission gas behaviour in oxide fuel for advanced engineering tools. Journal of Nuclear Materials, 502:323-330, 2018.

[150] Davide Pizzocri. Private Communication, 2018.

[151] Michael Polanyi. Über eine Art Gitterstörung, die einen Kristall plastisch machen könnte. Zeitschrift für Physik, 89(9-10):660-664, 1934. in German. 
[152] W. Püschl. Models for dislocation cross-slip in close-packed crystal structures: a critical review. Progress in Materials Science, 47(4):415 - 461, 2002.

[153] Python Software Foundation. The python standard library. https://docs. python.org/2/library/index.html, 2018. Accessed: 2018-07-18.

[154] D Raabe. Simulation of rolling textures of BCC metals considering grain interactions and crystallographic slip on $\{110\},\{112\}$ and $\{123\}$ planes. Materials Science and Engineering: A, 197(1):31-37, 1995.

[155] D Raabe, D Ma, and F Roters. Effects of initial orientation, sample geometry and friction on anisotropy and crystallographic orientation changes in single crystal microcompression deformation: A crystal plasticity finite element study. Acta Materialia, 55(13):4567-4583, 2007.

[156] J.N. Reddy. An Introduction to Continuum Mechanics. Cambridge University Press, 2 edition, 2013.

[157] C. Reuber, P. Eisenlohr, F. Roters, and D. Raabe. Dislocation density distribution around an indent in single-crystalline nickel: Comparing nonlocal crystal plasticity finite-element predictions with experiments. Acta Materialia, 71:333-348, 2014.

[158] M Rhee, H M Zbib, J P Hirth, H Huang, and T de la Rubia. Models for 
long-/short-range interactions and cross slip in $3 \mathrm{~d}$ dislocation simulation of bcc single crystals. Modelling and Simulation in Materials Science and Engineering, 6(4):467, 1998 .

[159] James R Rice. Inelastic constitutive relations for solids: an internal-variable theory and its application to metal plasticity. Journal of the Mechanics and Physics of Solids, 19(6):433-455, 1971.

[160] F Roters, D Raabe, and G Gottstein. Work hardening in heterogeneous alloysa microstructural approach based on three internal state variables. Acta materialia, 48(17):4181-4189, 2000.

[161] Franz Roters, Philip Eisenlohr, Luc Hantcherli, Denny Dharmawan Tjahjanto, Thomas R Bieler, and Dierk Raabe. Overview of constitutive laws, kinematics, homogenization and multiscale methods in crystal plasticity finite-element modeling: Theory, experiments, applications. Acta Materialia, 58(4):1152$1211,2010$.

[162] TJ Ruggles and DT Fullwood. Estimations of bulk geometrically necessary dislocation density using high resolution ebsd. Ultramicroscopy, 133:8-15, 2013.

[163] A. A. Salem, S. R. Kalidindi, and S. L. Semiatin. Strain hardening due to deformation twinning in $\alpha$-titanium: Constitutive relations and crystal-plasticity modeling. Acta Materialia, 53(12):3495-3502, 2005. 
[164] A. Sarkar, P. Mukherjee, P. Barat, T. Jayakumar, S. Mahadevan, and Sanjay K. Rai. Lattice Misfit Measurement in Inconel 625 By X-Ray Diffraction Technique. International Journal of Modern Physics B, 22(23):3977-3985, 2008 .

[165] Jérôme Serp, Michel Allibert, Ondřej Beneš, Sylvie Delpech, Olga Feynberg, Véronique Ghetta, Daniel Heuer, David Holcomb, Victor Ignatiev, Jan Leen Kloosterman, et al. The molten salt reactor (msr) in generation iv: overview and perspectives. Progress in Nuclear Energy, 77:308-319, 2014.

[166] Kazuyuki Shizawa and HM Zbib. A thermodynamical theory of gradient elastoplasticity with dislocation density tensor. i: Fundamentals. International Journal of Plasticity, 15(9):899-938, 1999.

[167] BN Singh, AJE Foreman, and H Trinkaus. Radiation hardening revisited: role of intracascade clustering. Journal of Nuclear Materials, 249(2-3):103-115, 1997.

[168] William S. Slaughter. The Linearized Theory of Elasticity. Birkhäuser, 2002.

[169] Jin E. Song and David L. Mcdowell. Grain Scale Crystal Plasticity Model with Slip and Microtwinning for a Third Generation Ni-Base Disk Alloy. Superalloys 2012, pages 159-166, 2012. 
[170] BW Spencer, WM Hoffman, and MA Backman. Modular system for probabilistic fracture mechanics analysis of embrittled reactor pressure vessels in the grizzly code. Nuclear Engineering and Design, 341:25-37, 2019.

[171] P. Steinmann. Views on multiplicative elastoplasticity and the continuum theory of dislocations. International Journal of Engineering Science, 34(15):1717$1735,1996$.

[172] B. Stephan, D. Jacob, F. Delabrouille, and L. Legras. A kinetic study of order-disorder transition in Ni-Cr based alloys. Minerals, Metals and Materials Series, pages 233-249, 2018.

[173] Roger E Stoller. The role of cascade energy and temperature in primary defect formation in iron. Journal of Nuclear Materials, 276(1-3):22-32, 2000.

[174] S. Sun, B. L. Adams, and W. E. King. Observations of lattice curvature near the interface of a deformed aluminium bicrystal. Philosophical Magazine A: Physics of Condensed Matter, Structure, Defects and Mechanical Properties, $80(1): 9-25,2000$.

[175] M. Sundararaman, Lalit Kumar, G. Eswara Prasad, P. Mukhopadhyay, and S. Banerjee. Precipitation of an intermetallic phase with Pt2Mo-type structure in alloy 625. Metallurgical and Materials Transactions A: Physical Metallurgy and Materials Science, 30(1):41-52, 1999. 
[176] Peter Szelestey, Marco Patriarca, and Kimmo Kaski. Computational study of core structure and peierls stress of dissociated dislocations in nickel. Modelling and Simulation in Materials Science and Engineering, 11(6):883, 2003.

[177] Nasrin Taheri-Nassaj and Hussein M Zbib. A mesoscale model of plasticity: Dislocation dynamics and patterning (one-dimensional). Journal of Engineering Materials and Technology, 138(4):041015, 2016.

[178] Tomoyuki Takeuchi. Work Hardening of Copper Single Crystals with Multiple Glide Orientations. Transactions of the Japan Institute of Metals, 16(10):629640, 1975.

[179] Geoffrey I. Taylor. The mechanism of plastic deformation of crystals. part i.theoretical. Proceedings of the Royal Society A, 145(855):362-387, 1934.

[180] Geoffrey I. Taylor. Plastic strain in metals. Journal of the Institute of Metals, 62:307-324, 1938.

[181] Fei Teng. Private Communication, 2018.

[182] Fei Teng. Investigation of thermal degradation in structural alloys for nuclear power systems. PhD thesis, Oregon State University, 2018.

[183] Fei Teng and Julie D. Tucker. Role of stoichiometry on ordering in Ni-Cr 
alloys. In Materials Research Society Symposium Proceedings, volume 1809, pages 7-12. Materials Research Society, 2015.

[184] Charles Thomas and Peter Tait. The performance of Alloy 625 in long-term intermediate temperature applications. International Journal of Pressure Vessels and Piping, 59(1-3):41-49, 1994.

[185] C. N. Tomé, R. A. Lebensohn, and U. F. Kocks. A model for texture development dominated by deformation twinning: Application to zirconium alloys. Acta Metallurgica Et Materialia, 39(11):2667-2680, 1991.

[186] Richard A Toupin. Elastic materials with couple-stresses. Archive for Rational Mechanics and Analysis, 11(1):385-414, 1962.

[187] Clifford Truesdell and Walter Noll. The Non-Linear Field Theories of Mechanics. Springer, 3 edition, 2004.

[188] Julie Tucker. Private Communication, 2018.

[189] Julie Tucker, Emmanuelle Marquis, Benjamin Spencer, Grace Burke, Fei Teng, Li-Jen Yu, Stephanie Pitts, and Octav Ciuca. Modeling and validation of irradiation damage in ni-based alloys for long- term lwr applications. Technical report, Oregon State University, 2107. Year 2 Annual Report for U.S. Department of Energy Nuclear Energy University Partnerships Grant. 
[190] Michael D Uchic, Dennis M Dimiduk, Jeffrey N Florando, and William D Nix. Sample dimensions influence strength and crystal plasticity. Science, 305(5686):986-989, 2004.

[191] RR Unocic, L Kovarik, C Shen, PM Sarosi, Y Wang, J Li, S Ghosh, and MJ Mills. Deformation mechanisms in ni-base disk superalloys at higher temperatures. Superalloys, 8:377, 2008.

[192] U.S. Energy Information Administration. Nuclear explained: Data \& statistics. Technical report, U.S. Energy Information Administration, September 2018.

[193] A. Verma, J. B. Singh, N. Wanderka, and J. K. Chakravartty. Delineating the roles of $\mathrm{Cr}$ and Mo during ordering transformations in stoichiometric $\mathrm{Ni} \$ 2 \$\left(\mathrm{Cr} \$-\{1-\mathrm{x}\} \$, \mathrm{Mo} \$ \_\mathrm{x} \$\right)$ alloys. Acta Materialia, 96:366-377, 2015.

[194] Gary S Was. Fundamentals of Radiation Materials Science: Metals and Alloys. Springer, 2007.

[195] R. L. Williamson, J. D. Hales, S. R. Novascone, M. R. Tonks, D. R. Gaston, C. J. Permann, D. Andrs, and R. C. Martineau. Multidimensional multiphysics simulation of nuclear fuel behavior. Journal of Nuclear Materials, 423:149-163, 2012.

[196] BD Wirth, MJ Caturla, T Díaz De La Rubia, T Khraishi, and H Zbib. Me- 
chanical property degradation in irradiated materials: A multiscale modeling approach. Nuclear Instruments and Methods in Physics Research Section B: Beam Interactions with Materials and Atoms, 180(1-4):23-31, 2001.

[197] Su Leen Wong, Manjunatha Madivala, Ulrich Prahl, Franz Roters, and Dierk Raabe. A crystal plasticity model for twinning- and transformation-induced plasticity. Acta Materialia, 118:140-151, 2016.

[198] Xianping Wu, Surya R Kalidindi, Carl Necker, and Ayman A Salem. Prediction of crystallographic texture evolution and anisotropic stress-strain curves during large plastic strains in high purity $\alpha$-titanium using a taylor-type crystal plasticity model. Acta Materialia, 55(2):423-432, 2007.

[199] Ritsuo Yoshioka, Motoyasu Kinoshita, and Ian Scott. Materials, pages 189 207. Woodhead Publishing, 2017.

[200] George Young, Julie Tucker, and Daniel Eno. The kinetics of long range ordering in ni-cr alloys. In 16th International Conference on Environmental Degradation of Materials in Nuclear Power Systems - Water Reactors, 2013.

[201] Daniel R. Young, George A Eno. Long Range Ordering in Model Ni-Cr-X Alloys. In Contribution of Materials Investigations and Operating Experience to LWRs' Safety, Performance, and Reliability, Avignon, France, 2014. 
[202] H. M. Zbib and E. C. Aifantis. Size effects and length scales in gradient plasticity and dislocation dynamics. Scripta Materialia, 48(2):155-160, 2003.

[203] H. M. Zbib and J. S. Jubran. Dynamic shear banding: a three-dimensional analysis. International Journal of Plasticity, 8(6):619-641, 1992.

[204] Hussein M. Zbib, Tomas Díaz De La Rubia, Moono Rhee, and John P. Hirth. 3D dislocation dynamics: Stress-strain behavior and hardening mechanisms in FCC and BCC metals. Journal of Nuclear Materials, 276(1):154-165, 2000.

[205] A Zervos, S-A Papanicolopulos, and I Vardoulakis. Two finite-element discretizations for gradient elasticity. Journal of engineering mechanics, 135(3):203-213, 2009.

[206] Xu Zhang, Katerina E Aifantis, and Alfonso HW Ngan. Interpreting the stressstrain response of al micropillars through gradient plasticity. Materials Science and Engineering: A, 591:38-45, 2014.

[207] Yongfeng Zhang, Paul C Millett, Michael R Tonks, Xian-Ming Bai, and S Bulent Biner. Molecular dynamics simulations of intergranular fracture in uo2 with nine empirical interatomic potentials. Journal of Nuclear Materials, 452(1-3):296-303, 2014.

[208] O. C. Zienkiewicz and J. Z. Zhu. The superconvergent patch recovery and 
a posteriori error estimates. Part 1: The recovery technique. International Journal for Numerical Methods in Engineering, 33(7):1331-1364, 1992.

[209] Steven J Zinkle and Nasr M Ghoniem. Prospects for accelerated development of high performance structural materials. Journal of Nuclear Materials, 417(13):2-8, 2011.

[210] Steven J Zinkle and Yoshi Matsukawa. Observation and analysis of defect cluster production and interactions with dislocations. Journal of Nuclear Materials, 329:88-96, 2004. 